OECD Health Working Papers No. 29

\title{
Health Care Quality Indicators Project 2006 Data Collection Update Report
}

Sandra Garcia Armesto, Maria Luisa Gil Lapetra, Lihan Wei, Edward Kelley 
Organisation de Coopération et de Développement Economiques

Organisation for Economic Co-operation and Development

30-Oct-2007

DIRECTORATE FOR EMPLOYMENT, LABOUR AND SOCIAL AFFAIRS

English - Or. English HEALTH COMMITTEE

Health Working Papers

OECD HEALTH WORKING PAPERS NO. 29

HEALTH CARE QUALITY INDICATORS PROJECT 2006 DATA COLLECTION UPDATE REPORT

Sandra Garcia Armesto, Maria Luisa Gil Lapetra, Lihan Wei, Edward Kelley and the Members of the HCQI Expert Group

This version has been amended to correct mis-statements in the Acknowledgments page. 


\title{
DIRECTORATE FOR EMPLOYMENT, LABOUR AND SOCIAL AFFAIRS
}

\section{OECD HEALTH WORKING PAPERS}

This series is designed to make available to a wider readership health studies prepared for use within the OECD. Authorship is usually collective, but principal writers are named. The papers are generally available only in their original language - English or French - with a summary in the other.

Comment on the series is welcome, and should be sent to the Directorate for Employment, Labour and Social Affairs, 2, rue André-Pascal, 75775 PARIS CEDEX 16, France.

The opinions expressed and arguments employed here are the responsibility of the author(s) and do not necessarily reflect those of the OECD

\author{
Applications for permission to reproduce or translate \\ all or part of this material should be made to: \\ Head of Publications Service \\ OECD \\ 2, rue André-Pascal \\ 75775 Paris, CEDEX 16 \\ France
}

Copyright OECD 2007 


\section{ACKNOWLEDGEMENTS}

1. The Health Care Quality Indicators Project was guided by an expert group made up of national experts nominated by countries participating in the project ${ }^{1}$. Financial support from the European Commission was in part used to support outreach to non-OECD European member states to encourage their participation in the collection of data. To date, three of these eight countries supplied data : Cyprus ${ }^{2,3}$, Latvia and Malta.

2. The authors would like to acknowledge the input provided by Peter Scherer, Elizabeth Docteur, Jeremy Hurst, Niek Klazinga and Gaetan Lafortune.

3. The HCQI project was funded during 2006 partly by regular contributions from member countries of the OECD. Additional voluntary contributions to the project were made by the following member countries: Australia, Denmark, Ireland, Italy, Japan, Netherlands, New Zealand, Norway, and Spain. In addition, 'in kind' support was provided by the United States through the secondment of Dr. Edward Kelley from the Agency for Healthcare Research and Quality (AHRQ) to the project throughout 2006. Support was also provided by the Commonwealth Fund during the first phase of the HCQI Project that made possible the appointment of Professor Arnie Epstein (Harvard University) as chairman of the project's Expert Group during that period

4. The HCQI project was also supported during 2006 by a grant provided by the European Commission (DG SANCO).

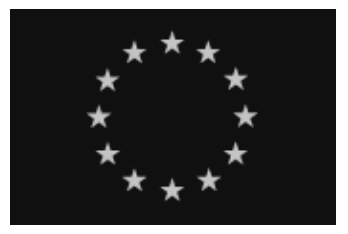

\footnotetext{
${ }^{1}$ The following OECD countries participate in the Group:

$\begin{array}{llllll}\text { Australia, } & \text { Denmark, } & \text { Hungary, } & \text { Korea, } & \text { Poland, } & \text { Switzerland, } \\ \text { Austria, } & \text { Finland, } & \text { Iceland, } & \text { Mexico, } & \text { Portugal, } & \text { Turkey, } \\ \text { Belgium, } & \text { France, } & \text { Ireland, } & \text { Netherlands, } & \text { Slovak Republic, } & \text { United Kingdom, } \\ \text { Canada, } & \text { Germany, } & \text { Italy, } & \text { New Zealand, } & \text { Spain, } & \text { United States. } \\ \text { Czech Republic, } & \text { Greece, } & \text { Japan, } & \text { Norway, } & \text { Sweden, } & \end{array}$
}

${ }^{2}$ Footnote by Turkey:

The information in this document with reference to «Cyprus » relates to the southern part of the Island. There is no single authority representing both Turkish and Greek Cypriot people on the Island. Turkey recognizes the Turkish Republic of Northern Cyprus (TRNC). Until a lasting and equitable solution is found within the context of United Nations, Turkey shall preserve its position concerning the "Cyprus issue".

${ }^{3}$ Footnote by all the European Union Member States of the OECD and the European Commission:

The Republic of Cyprus is recognized by all members of the United Nations with the exception of Turkey. The information in this document relates to the area under the effective control of the Government of the Republic of Cyprus. 
DELSA/HEA/WD/HWP(2007)4 


\section{SUMMARY}

5. This report is an update to the OECD Health Working Paper No. 22, Health Care Quality Indicators Project: Initial Indicators Report that was based on data collected between 2003 and 2005 and released in $2006^{4}$. That report presented the OECD's initial work on developing a set of health care quality indicators that could be used to raise questions about differences in quality of care across countries. The 2006 report covered 21 "initial indicators" with data provided by 24 countries. It identified 17 of these indicators as being fit for international comparisons of which 4 were identified as needing further work ${ }^{5}$. Following the release of that report in March 2006, the OECD undertook a second round of data collection on the initial indicator set and also gathered data for the first time on new indicators in a questionnaire sent to participating HCQI countries. This paper reports on the results of that second round of data collection. Data is presented here on an augmented indicator set considered fit for the purpose of making international comparisons on quality of health care. The data is comprised of 19 indicators $(17$ initial indicators plus 2 new ones). The paper also presents the data provided on 7 other indicators that are not yet considered fit for international comparison. In this round of data collection, data were reported by 32 countries.

6. The Secretariat and collaborating HCQI country experts carried out several data comparability analyses on the new indicators for which data had been gathered. Certain comparability issues were discussed with the HCQI Project Expert Group at its meeting in Paris in October 2006. These issues included:

- The use of data which were not nationally representative

- Presentation of administrative versus survey data for cancer screening

- Harmonising data recall periods for cancer survival and screening

- The use of a truncated standard population for age adjustment

7. The results of the above analysis are presented in detail in this paper. However, an overall view of data comparability and possible improvement can be offered. It is clear from the analysis that significant progress has been made within the HCQI Project and by individual member countries in improving data comparability on the set of indicators from the HCQI Project since the first data collection in 2003. For example, on a range of survey indicators (such as cancer screening), countries have been able to alter national reporting standards to provide the OECD with comparable data. In the area of mortality rates for

\footnotetext{
${ }^{4}$ Mattke S, Kelley E, Scherer P, Hurst J, Gil Lapetra M and the Members of the HCQI Expert Group (2006), "Health Care Quality Indicators Project: Initial Indicators Report", OECD Health Working Paper No. 22, OECD, Paris, http://www.oecd.org/dataoecd/1/34/36262514.pdf.

${ }^{5}$ The 21 indicators (those labelled "fit for international comparison" and "evaluated as not fit" in column 2 of Table 2 , below) were described as "17" indicators in OECD Health Working Paper No. 22. That is because a group of three related indicators for "incidence of vaccine preventable diseases" and a group of three related indicators for "coverage for basic vaccination programme" were each listed as one indicator respectively. The six components of these two groups are now considered separately.
} 


\section{DELSA/HEA/WD/HWP(2007)4}

acute myocardial infarction (AMI) and stroke, country estimates have become significantly more comparable since the first data collection. What is also clear is that both gaps in data and persistent data comparability issues remain within the indicator set. For example, there are a number of countries where nationally representative data for indicators such as cancer survival are not yet available. In other instances, countries have not been able to furnish the OECD with indicators that match the age or recall period (for survey data) specifications, making differences between countries difficult to interpret. However, member countries continue to be supportive of the Secretariat's efforts to investigate these data comparability issues. With this support, the Secretariat plans to continue to improve the existing indicators while at the same time work on developing new indicators based on comparable data.

8. As noted above, this paper presents three groups of indicators:

a) All of the initial 17 indicators that were reported as being fit for international comparison in Health Working Paper No. 22, which were updated during the 2006 data collection. They are:

- Breast cancer five-year survival rate

- Mammography screening rate

- Cervical cancer five-year survival rate

- Cervical cancer screening rate

- Colorectal cancer five-year survival rate

- Incidence of vaccine preventable diseases (Pertussis, measles, and hepatitis B)

- $\quad$ Coverage for basic vaccination programme, age 2, (Pertussis, measles, and hepatitis B)

- Asthma mortality rate, ages 5-39

- In-hospital mortality rate within 30 days of hospital admission for acute myocardial infarction

- In-hospital mortality rate within 30 days of hospital admission for stroke

- Waiting times for surgery after hip fracture, over age 65

- Influenza vaccination, over age 65

- Smoking rate

b) Following discussions from the October 2006 meeting, two new indicators tested in the 2006 data collection round that, were judged by the HCQI Expert Group as mature enough to be added to the initial set:

- Retinal exams in diabetics - This indicator was included in the first HCQI questionnaires (in 2004 and 2005) but was not considered fit until the 2006 data collection round.

- Asthma admission rate - This indicator was collected for the first time through the 2006 questionnaire.

c) Seven indicators were not yet considered suitable for inclusion in the HCQI data set for international comparison but were recommended for additional sensitivity analysis to find ways to improve 
comparability. Among them, three indicators were collected in the 2003-2005 questionnaire have seen no substantial improvements for comparability and thus are still considered not yet suitable for international comparison. These are:

- Annual HbAlc testing for patients with diabetics

- Patients with diabetics with poor glucose control

- Major amputations in diabetics

9. Four other indicators were collected for the first time through the 2006 questionnaire:

- $\quad$ Post-operative hip fracture or fall

- Transfusion reaction

- Uncontrolled diabetes admission rate

- Hypertension admission rate

10. The first group of indicators listed above is presented with definitions and updated data. For the second and third groups of indicators, the report reviews detailed information on the scientific soundness, importance, availability of data and the international comparability of the data for each indicator.

11. The smoking rate remains in the indicator set. This indicator was initially adopted since it is the risk factor which countries attempt to affect as they institute efforts through the health care delivery system to change tobacco consumption. There has been much discussion on this indicator because of its dependence on certain factors outside the control of the health system. It is therefore considered a relatively less valid indicator of quality of health care than the other indicators considered 'fit for international comparisons'. The Expert Group will examine other, more health care-related indicators of smoking cessation. At this point, however, these indicators are not available from a wide enough group of countries. Therefore, for the moment, the smoking rate continues to be retained among the 19 indicators in the 2006 HCQI indicator set. 


\section{RESUMÉ}

12. Le présent rapport est une version actualisée du Document de travail de l'OCDE sur la santé n 22 intitulé Health Care Quality Indicators Project: Initial Indicators Report, établi sur la base des données rassemblées en 2003/2005 et publié en $2006^{6}$. Ce rapport présentait les travaux initiaux de l'OCDE concernant l'élaboration d'une série d'indicateurs sur la qualité des soins de santé qui pourraient être utilisés pour tenter d'expliquer les différences en matière de qualité de soins entre les pays. Le rapport 2006 portait sur 21 «indicateurs initiaux» pour lesquels 24 pays avaient communiqué des données; il a été estimé que 17 de ces indicateurs se prêtaient à des comparaisons internationales et que quatre d'entre eux nécessitaient des travaux approfondis ${ }^{7}$. A la suite de la publication du rapport en mars 2006, l'OCDE a entamé un deuxième cycle de collecte de données relatives à la série initiale d'indicateurs et a entrepris de recueillir pour la première fois des données sur de nouveaux indicateurs par le biais d'un questionnaire adressé aux pays participants au projet HCQI. Le présent rapport fait état des résultats du deuxième cycle de collecte de données. Il contient des données sur la série élargie d'indicateurs considérés comme se prêtant à des comparaisons internationales, soit des données portant sur 19 indicateurs (17 indicateurs existants et 2 nouveaux). Il présente également les données fournies en ce qui concerne 7 autres indicateurs dont on estime qu'ils ne se prêtent pas encore à des comparaisons internationales. Les données communiquées émanent cette fois de 32 pays (des pays de l'UE qui ne sont pas membres de l'OCDE ont été invités à participer au projet ${ }^{8}$ ).

13. Dans le cadre de l'étude des nouveaux indicateurs au sujet desquels des données ont été réunies, le Secrétariat et les experts nationaux collaborant au projet HCQI ont mené plusieurs analyses touchant la comparabilité des données. Certaines questions de comparabilité ont été examinées avec le Groupe d'experts du Projet HCQI lors de la réunion de ce dernier à Paris en octobre 2006. Il s'agit des questions suivantes :

- L'utilisation de données non représentatives au plan national

- La présentation de données administratives par opposition à des données d'enquêtes en ce qui concerne le dépistage du cancer

\footnotetext{
${ }^{6}$ Mattke S, Kelley E, Scherer P, Hurst J, Gil Lapetra M et les membres du Groupe d'experts du Projet HCQI. Health Care Quality Indicators Project: Initial Indicators Report. Document de travail sur la santé no. 22. (Paris, France: OCDE). 2006. http://www.oecd.org/dataoecd/1/34/36262514.pdf.

${ }^{7}$ Les 21 indicateurs (voir la colonne 1 du tableau 2) étaient "en fait" présentés comme "17" indicateurs dans le Document de travail de l'OCDE sur la santé no. 22. En effet un groupe de trois indicateurs relatifs à l' « incidence des maladies pouvant être prévenues par la vaccination" et un groupe de trois indicateurs relatifs à la "couverture des programmes de vaccination de base" apparaissaient respectivement dans le document en tant qu'un seul indicateur. Les six indicateurs considérés dans ces deux groupes sont désormais distincts.

${ }^{8}$ Un appui financier de la Commission européenne a été utilisé afin de favoriser l'ouverture vers des États européens non membres de l'OCDE et d'encourager leur participation au projet. A ce jour, trois des huit pays concernés prennent désormais part au projet.
} 
- L'harmonisation des périodes de référence des données relatives au taux de survie et au dépistage du cancer

- L'utilisation d'une population standard tronquée à des fins d'ajustement en fonction de l'âge

14. Les résultats des analyses susmentionnées sont présentés de façon détaillée dans le rapport. Cela étant, on peut d'ores donner une idée générale de la comparabilité des données et dire si celle-ci s'est améliorée. Il ressort clairement de l'analyse des questions de comparabilité des données existantes et des données nouvelles que d'importants progrès ont été réalisés dans le cadre du Projet HCQI ainsi que par certains pays membres en ce qui concerne la comparabilité des données afférentes à l'ensemble des indicateurs considérés dans le Projet HCQI depuis la première collecte effectuée en 2003. Par exemple, s'agissant d'un ensemble d'indicateurs reposant sur des enquêtes (tel que le dépistage du cancer), les pays ont été à même de modifier leurs normes nationales de notification afin de fournir à l'OCDE des données comparables. Pour ce qui est des taux de mortalité après un infarctus aigu du myocarde ou après un accident vasculaire cérébral, la comparabilité des estimations des pays s'est beaucoup améliorée depuis la première collecte de données. Il apparaît par ailleurs clairement que certains problèmes de comparabilité des données concernant la série d'indicateurs retenus dans le projet HCQI n'ont pu encore être résolus. Ainsi, dans un certain nombre d'entre eux, des données représentatives au plan national relatif à des indicateurs tels que le taux de survie au cancer ne sont pas encore disponibles. Dans d'autres cas, les pays n'ont pas été en mesure de communiquer à l'OCDE des données correspondant aux spécifications en matière d'âge ou de période de référence (pour les données d'enquêtes), d'où des difficultés pour interpréter les différences entre pays. Cela étant, les pays membres s'intéressent à la comparabilité des données et ont soutenu les efforts déployés par le Secrétariat pour étudier les questions s'y rapportant. Grâce à ce soutien, le Secrétariat envisage d'affiner les indicateurs existants tout en travaillant à l'élaboration de nouveaux indicateurs s'appuyant sur des données comparables.

15. Comme on l'a vu ci-dessus, trois groupes d'indicateurs sont présentés dans le rapport:

a) L'ensemble des 17 indicateurs initiaux considérés comme se prêtant à des comparaisons internationales dans le Document de travail sur la santé $\mathrm{n}^{\circ} 22$, mis à jour à l'occasion de la collecte de données de 2006. Il s'agit des indicateurs suivants :

- Taux de survie à cinq ans au cancer du sein

- Taux de dépistage par mammographie

- Taux de survie à cinq ans au cancer du col de l'utérus

- Taux de dépistage du cancer du col de l'utérus

- Taux de survie à cinq ans au cancer colorectal

- Incidence des maladies pouvant être prévenues par la vaccination (coqueluche, rougeole et hépatite B)

- Couverture des programmes de vaccination de base, à l'âge de deux ans, (coqueluche, rougeole et hépatite B)

- Taux de mortalité pour cause d'asthme entre 5 et 39 ans

- Taux de mortalité à 30 jours hors hôpital/à l'hôpital après un infarctus aigu du myocarde

- Taux de mortalité à 30 jours hors hôpital/à l'hôpital après un accident vasculaire cérébral

- Temps d'attente pour une opération après une fracture de la hanche, à 65 ans et plus

- Vaccination contre la grippe pour les adultes de plus de 65 ans 
- Taux de tabagisme

b) Deux nouveaux indicateurs, testés lors du cycle de collecte de données de 2006 et jugés par le Groupe d'experts du Projet HCQI, à la suite des débats qui se sont déroulés lors de la réunion d'octobre 2006, comme étant suffisamment élaborés pour être ajoutés à l'ensemble initial.

- Examen de la rétine chez les patients diabétiques - cet indicateur figurait dans les questionnaires initiaux du Projet HCQI (en 2004 et 2005) mais n'était pas considéré comme approprié jusqu'à présent.

- Taux d'hospitalisation des adultes pour cause d'asthme - des données relatives à cet indicateur ont été collectées pour la première fois dans le questionnaire de 2006.

c) Sept indicateurs envisagés dans le projet HCQI dont on estime qu'ils ne sont pas prêts à être inclus dans la série car ils ne se prêtent à des comparaisons internationales, mais pour lesquels il a été recommandé de procéder à des analyses de sensibilité supplémentaires afin de trouver des moyens d'améliorer la comparabilité des données. Des données ont été rassemblées sur trois d'entre eux pour les questionnaires de 2003 à 2005 mais, faute d'une amélioration sensible de leur comparabilité, on considère que ces indicateurs ne se prêtent toujours pas à des comparaisons internationales. Il s'agit des indicateurs suivants :

- Dosage de l'HbA1c chez les personnes diabétiques

- Contrôle insuffisant de la glycémie chez les personnes diabétiques

- Amputations majeures chez les personnes diabétiques

16. Quatre d'entre eux ont fait l'objet d'une collecte de données pour la première fois dans le cadre du questionnaire de 2006 :

- Fracture de la hanche ou chute post-opératoire

- Réaction à la transfusion

- Taux d'hospitalisation pour diabète non contrôlé

- Taux d'hospitalisation pour hypertension

17. Les indicateurs du premier groupe sont accompagnés de leur définition et de données actualisées. S'agissant des indicateurs des deuxième et troisième groupes, le rapport examine des informations détaillées concernant la validité scientifique, l'importance et la disponibilité des données ainsi que la comparabilité internationale de ces dernières pour chacun d'eux.

18. Le taux de tabagisme continue de figurer dans la série d'indicateurs du Projet HCQI considérée dans le présent rapport. Cet indicateur a été initialement retenu car il représente l'aspect essentiel sur lequel les pays s'efforcent d'influer lorsqu'ils s'attachent à modifier le comportement des fumeurs par le biais du système de soins de santé. Beaucoup de questions ont été soulevées au sujet de cet indicateur et du fait qu'il dépend de nombreux facteurs qui échappent au contrôle du système de santé. Il s'agit donc d'un indicateur relativement moins représentatif de la qualité des soins de santé. Le Groupe d'experts examinera d'autres indicateurs concernant l'arrêt du tabac qui sont davantage liés aux soins de santé. Cela étant, à ce stade, ces indicateurs ne sont pas disponibles dans un groupe suffisamment important de pays. Le taux de tabagisme continue donc de figurer au nombre des 19 indicateurs de la série retenue pour 2006 dans le cadre du projet HCQI. 


\section{TABLE OF CONTENTS}

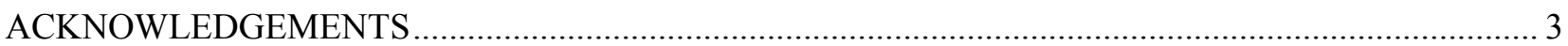

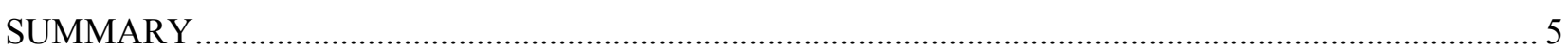

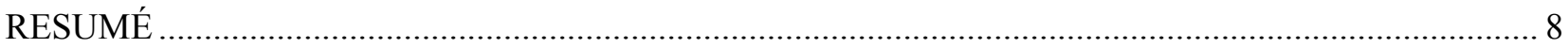

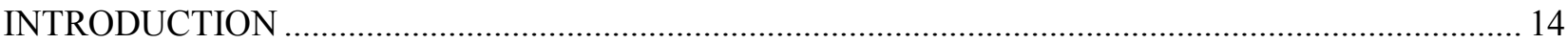

Health Care Quality Indicator Project: purpose and initial steps ........................................................ 14

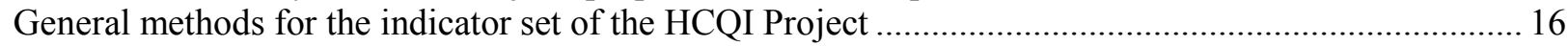

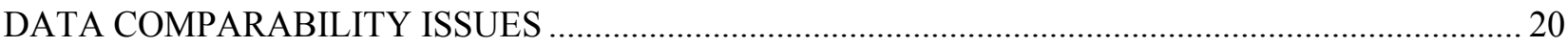

"INITIAL" (2003-2005) INDICATORS: 2006 SPECIFICATIONS AND DATA RESULTS ................... 24

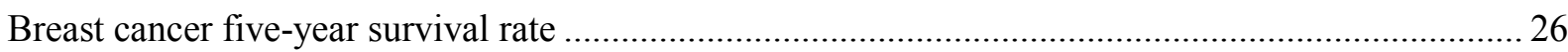

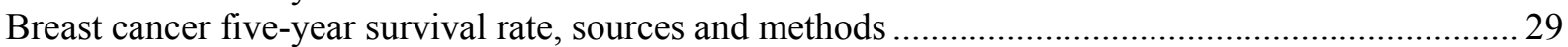

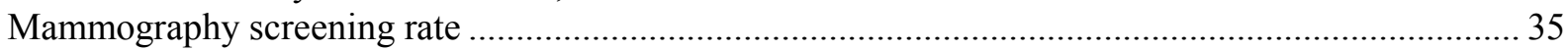

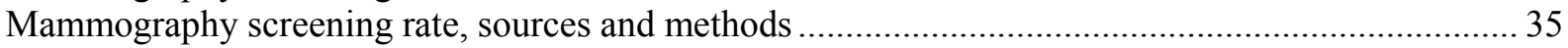

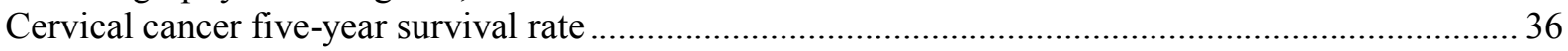

Cervical cancer five-year survival rate, sources and methods ............................................................ 39

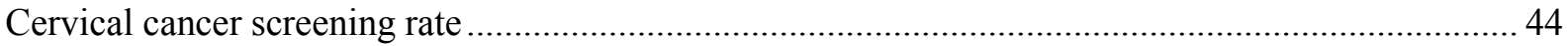

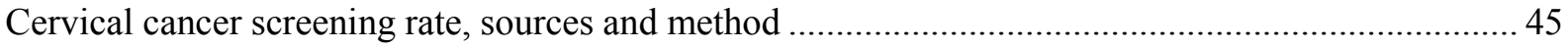

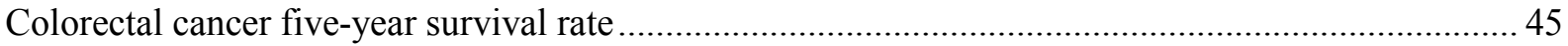

Colorectal cancer five-year survival rate, sources and methods....................................................... 48

Incidence of vaccine preventable diseases (Pertussis, measles, hepatitis B) ....................................... 52

Incidence of vaccine preventable diseases (Pertussis, measles, hepatitis B), sources and methods...... 54

Coverage for basic vaccination programme, age 2, (Pertussis, measles, hepatitis B) .......................... 60

Coverage for basic vaccination programme, age 2, (Pertussis, measles, hepatitis B), sources and

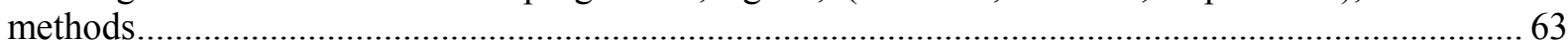

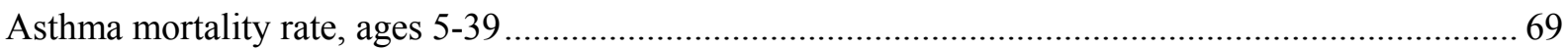

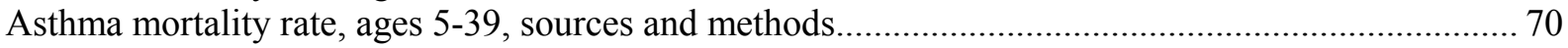

In-hospital mortality rate within 30 days of hospital admission for AMI ........................................ 73

In-hospital mortality rate within 30 days of hospital admission for AMI, sources and methods .......... 75

In-hospital mortality rate within 30 days of hospital admission for stroke ........................................ 79

In-hospital mortality rate within 30 days of hospital admission for stroke, sources and methods ........ 81

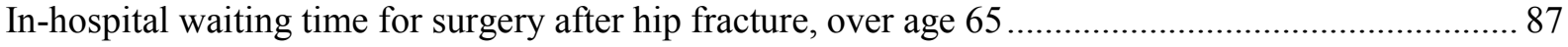

In-hospital waiting time for surgery after hip fracture, over age 65 , sources and methods.................. 88

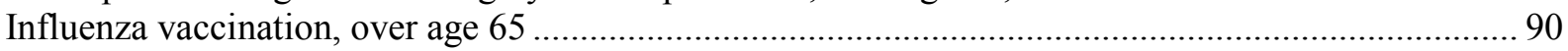

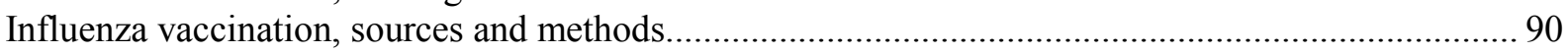

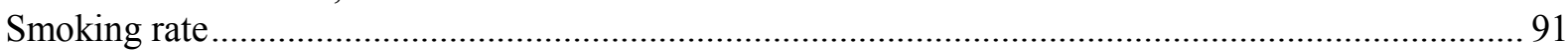

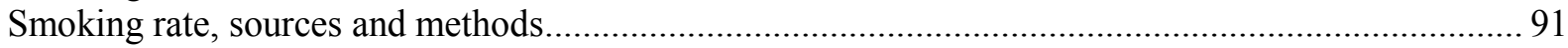


TWO NEW INDICATORS CONSIDERED FIT FOR INTERNATIONAL COMPARISONS: CLINICAL IMPORTANCE, SCIENTIFIC SOUNDNESS, SPECIFICATIONS AND DATA RESULTS ................... 92

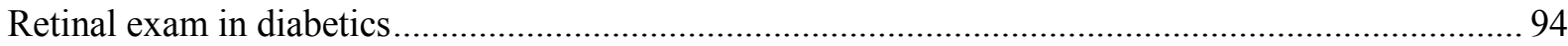

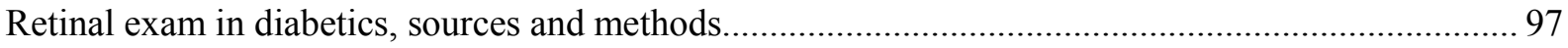

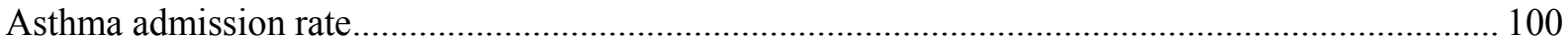

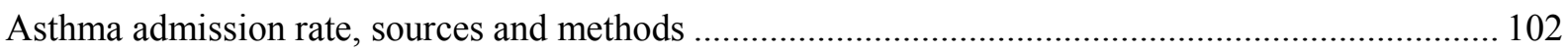

INDICATORS NOT SELECTED FOR INCLUSION IN 2006 HCQI INDICATOR SET ...................... 107

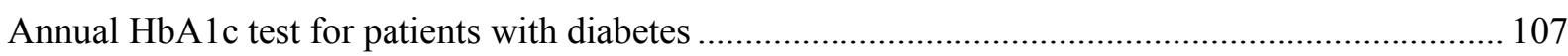

Annual $\mathrm{HbAl} \mathrm{c}$ test for patients with diabetes, sources and methods ............................................... 110

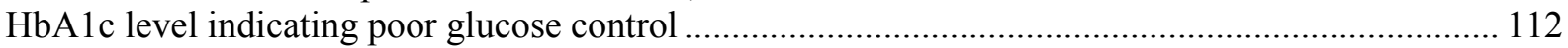

$\mathrm{HbA1}$ level indicating poor glucose control, sources and methods ................................................. 114

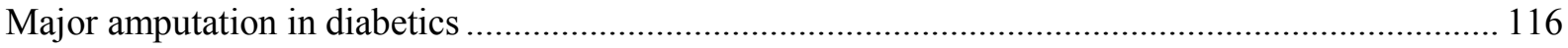

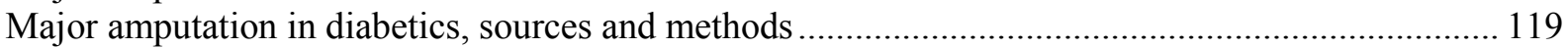

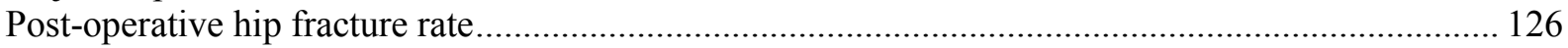

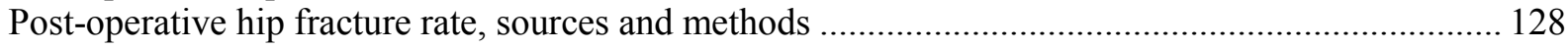

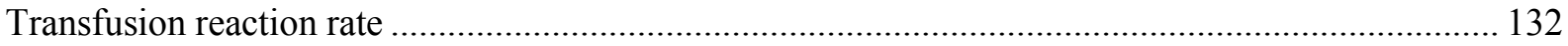

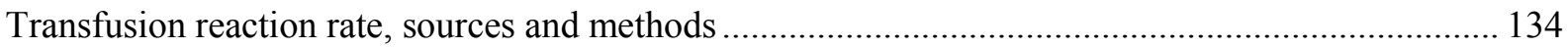

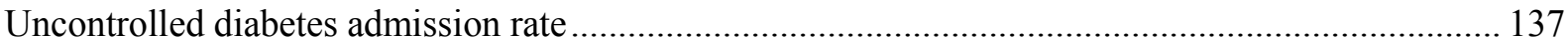

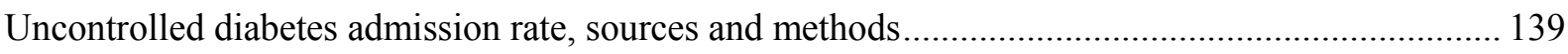

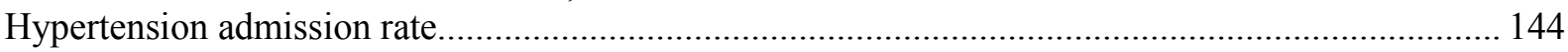

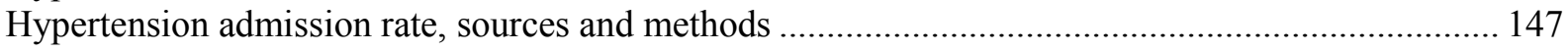

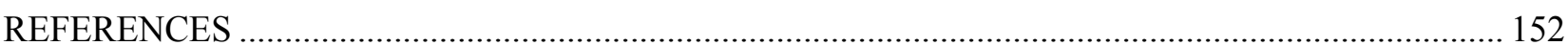

\section{Tables}

Table 1. Example of questionnaire response categories .............................................................. 17

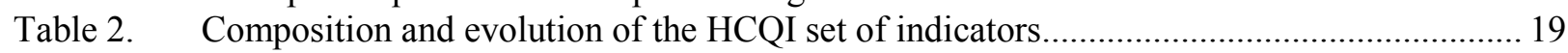

Table 3. 2004 Admission based ischemic stroke case fatality in Sweden ........................................ 23

Table 4. Availability of data for 'Initial' (2003-2005) Indicators presented in this section ............... 25

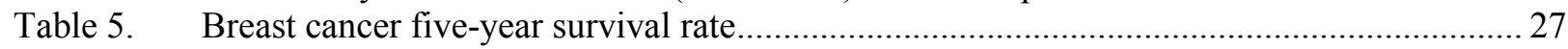

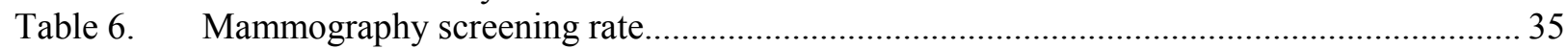

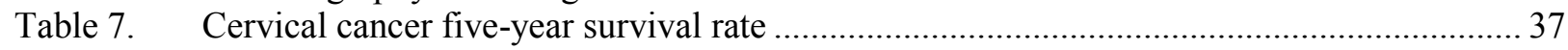

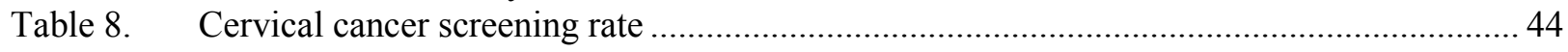

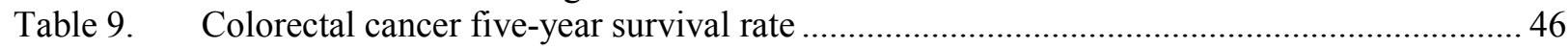

Table 10. Incidence of vaccine preventable diseases (Pertussis, measles, hepatitis B) ...................... 52

Table 11. Coverage for basic vaccination programme, age 2, (Pertussis, measles, hepatitis B) .......... 61

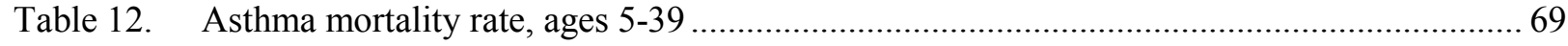

Table 13. In-hospital mortality rate within 30 days of hospital admission for AMI........................... 73

Table 14. In-hospital mortality rate within 30 days of hospital admission for stroke.......................... 79

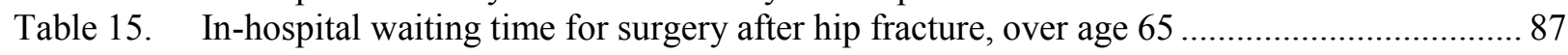

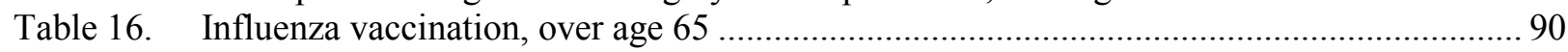

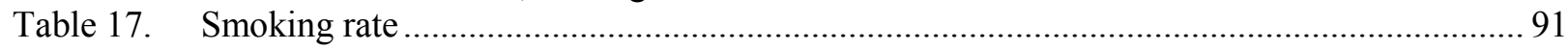

Table 18. Summary of data availability for two new indicators in

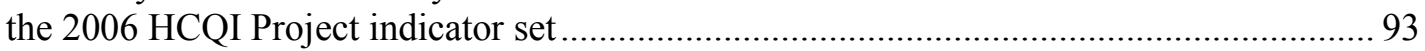

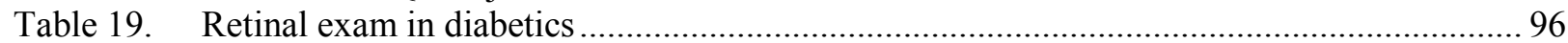

Table 20. Asthma admission rate per 10000 discharges, (primary care and prevention,

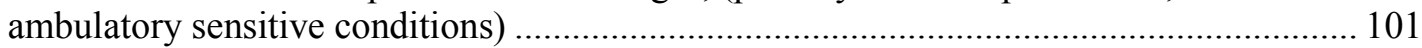

Table 21. Annual HbA1c test for patients with diabetes ............................................................. 109 
Table 22. Annual HbA1c test for patients with diabetes, comparability issues................................ 111

Table 23. HbA1c level indicating poor glucose control ................................................................. 113

Table 24. HbA1c level indicating poor glucose control, comparability issues.................................. 115

Table 25. Major amputation in diabetics, per 10000 diabetics ..................................................... 118

Table 26. Major amputation in diabetics, comparability issues......................................................... 125

Table 27. Post-operative hip fracture rate per 100 discharges ......................................................... 127

Table 28. Post-operative hip fracture rate, comparability issues ..................................................... 131

Table 29. Transfusion reaction rate per 100000 discharges........................................................... 133

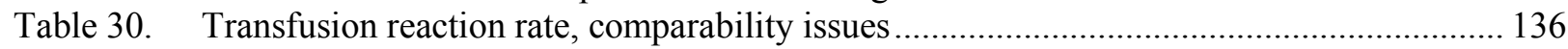

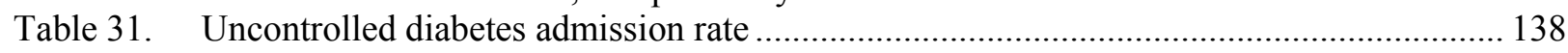

Table 32. Uncontrolled diabetes admission rate, comparability issues........................................... 143

Table 33. Hypertension admission rate per 100000 discharges ..................................................... 146

Table 34. Hypertension admission rate, comparability issues ........................................................ 151

\section{Figures}

Figure 1. Findings on differences between administrative and survey data for cancer screening ...... 21 


\section{INTRODUCTION}

\section{Health Care Quality Indicator Project: purpose and initial steps}

19. Quality of health care delivery is a topic of concern throughout the member countries of the OECD. Articles on shortcomings in care or on comparative differences in quality across countries have become more frequent in the popular press. Efforts to improve the measurement of quality of care through the development of quality indicators have become more present in the literature and in policy forums worldwide (IOM, 2001; Sawicki, 2005; Roland, 2004; AHRQ, 2005). Many of these efforts target specific disease areas in one particular country while others compare across countries, but target particular conditions (Ramirez, 2005). The quality indicator set reported on here is one of the few efforts ${ }^{9}$ which have attempted to examine quality of care across clinical conditions for more than one country.

20. The long-term objective of the Health Care Quality Indicator (HCQI) Project is to develop a set of indicators that can be used to raise questions on health care quality and that can be reliably reported across countries using comparable data. The indicators are intended to help raise questions for further investigations of differences in quality of care across countries. In light of its endeavour, the indicators of the HCQI Project are conceived as a "living set" in which indicators transit through different stages of maturity until they are considered fit for the purpose of international comparisons. These stages arrive from the joint effort between the participating countries and the Secretariat to improve the quality and the quantity of available data. At the core of the project rests the conviction that to enhance the international comparability of health care quality it is indispensable to have a thorough and methodical work plan for the refinement and standardisation of health care information along with the steady encouragement of information systems' development at the national level. Table 2 illustrates the process of broadening the set of indicators hitherto, showing the list of indicators involved in each data collection round and their assessed status in terms of fitness for use.

21. The OECD HCQI project began in 2001, building on two previous international initiatives to develop indicators of health care quality across countries. One of these, initiated by the Commonwealth Fund involved five countries (Australia, Canada, New Zealand, United Kingdom and United States). Another, initiated by the Nordic Council of Ministers, included another six countries (Denmark, Finland, Iceland, Norway, Sweden and Greenland). Initially a group of 19 countries (including ten of those listed under the two initiatives mentioned above) accepted the OECD's invitation to join the HCQI project. A preliminary list of indicators was derived from the work of these two earlier initiatives. Based on expert judgement, the HCQI Country Expert group chose 21 indicators out of that list, considering them as scientifically sound and important. These indicators had also shown an acceptable degree of comparability

9 First Report and Recommendations of the Commonwealth Fund's International Working Group on Quality Indicators a Report to Health Ministers of Australia, Canada, New Zealand, the United Kingdom, and the United States. June 2004. The Commonwealth Fund. (no. 752). 
across countries in the context of the previous exercises. Data collection for these 21 indicators was conducted across participating countries to prove its feasibility. Various checks were undertaken of the indicators' specifications across countries to establish their individual fitness for the purpose of international comparisons.

22. The indicator selection criteria applied in the process of creating the original and current indicator list are summarised here. For an indicator to be a useful tool for evidence-based policy decisions, two conditions should be met. First, it has to capture an important performance aspect. Second, it must be scientifically sound.

23. The importance of an indicator can be further broken down into three dimensions:

- Impact on health. What is the impact on health associated with this problem? Does the measure address areas in which there is a clear gap between the actual and potential levels of health? The impact on health is quantified where data is available for each indicator by using mortality and morbidity estimates from the World Health Organization for the 'EURO A ${ }^{10}$ group of countries, (Murray, 2001). This group of countries includes most of the countries participating in the OECD HCQI Project.

- Policy importance. Are policymakers and consumers concerned about this area? Although this dimension is difficult to quantify objectively, the cost associated with the condition covered by each indicator is used to indicate the economic importance related to each indicator. Where suitable evidence on costs exists, it is also presented for each indicator.

- Susceptibility to being influenced by the health care system. Can the health care system meaningfully address this aspect or problem? Does the health care system have an impact on the indicator independent of confounders like patient risk? Will changes in the indicator give information about success or failure of policy changes? This dimension is discussed based on a review of the relevant literature demonstrating that the health system can influence each indicator.

The scientific soundness of each indicator can also be broken down into three dimensions:

- Face validity. Does the measure make sense logically and clinically? The face validity of each indicator in this report is based on the basic clinical rationale for the indicator and on past usage of the indicator in national or other quality reporting activities.

- Content validity. Does the measure capture meaningful aspects of the quality of care? Content validity is assessed through a literature review of studies relevant to each indicator.

- Reliability. Does the measure provide stable results across various populations and circumstances? Reliability of each indicator is assessed through a literature review of studies assessing the stability of results across populations or circumstances.

24. The application of these criteria to the initial indicator set of the HCQI Project had been carried out as part of the two predecessor projects to the HCQI Project, mentioned above. For the Commonwealth Fund work, a rating system was used to rank each indicator based on the above criteria. Indicators which

\footnotetext{
${ }^{10}$ WHO EURO A countries include Andorra, Austria, Belgium, Croatia, Czech Republic, Denmark, Finland, France, Germany, Greece, Iceland, Ireland, Israel, Italy, Luxembourg, Malta, Monaco, Netherlands, Norway, Portugal, San Marino, Slovenia, Spain, Sweden, Switzerland, and the United Kingdom.
} 
ranked highly on these criteria were retained in the measure set. This rating process was reviewed by the OECD Secretariat and then by the HCQI Expert Group as it began its work on selecting indicators.

25. The initial gathering of information and analysis was carried out from 2003 to 2005; new countries have continued to join the project, providing additional data for the analysis. The indicators collected during this period are listed in the first column of Table 2. Of the indicators, 17 of the 21 tested were considered fit for the purpose of international comparisons, but 4 of them (those related to diabetes care) were regarded as not yet ready, either due to their lack of availability across a sufficient number of countries (the experts agreed on a threshold of at least 10 countries able to provide data on the indicator) or some comparability issues not yet overcome. The data collected for the 21 indicators, together with the analysis of their fitness for international comparisons, were published as OECD Health Working Paper No. 22, Health Care Quality Indicators Project: Initial Indicators Report (Mattke et al., 2006).

26. The set of indicators resulting from the initial compilation effort was judged by the HCQI Expert Group as being too limited for comprehensive comparisons of the quality of health care across countries. Therefore the HCQI Expert Group instituted a process to identify important gaps in the areas of health care for which indicators had been developed, judging by the burden of disease being tackled, health care utilisation rates and costs. Country experts were asked to rate a set of health care condition areas in terms of importance. This rating process yielded 5 priority areas for the development of additional HCQI indicators ${ }^{11}$ :

- Cardiac care

- Diabetes care

- Primary care and prevention

- Mental health

- Patient safety

27. Five international expert panels were commissioned to propose relevant and scientifically sound measures for each of these areas ${ }^{12}$. The result was a list of 86 indicators considered as valid and reliable to report on the quality of care in these priority areas.

\section{General methods for the indicator set of the HCQI Project}

28. Following the release of the five expert panel reports recommending the 86 indicators in the 5 priority areas, an availability survey was conducted across the participating countries to find out whether 10 or more countries would be able to collect the data for each indicator. The 86 indicators included a few already being collected in OECD Health Data as well as some being gathered as part of the initial indicator set at the time when the recommendations were issued.

\footnotetext{
${ }^{11}$ For a detailed description of this decision making process please consult the monographic supplement published by International Journal for Quality in Health Care; September 2006 Volume 18 supplement I.

${ }^{12}$ The five reports were published as OECD Health Technical Paper Nos. 14 - 18. The reports are downloadable from the HCQI web page www.oecd.org/health/hcqi. An overall picture of the process and a summary of the recommendations produced by the 5 panels can also be found in the monographic supplement published by the International Journal for Quality in Health Care, 2006, Vol.18 (suppl. 1).
} 
29. Questionnaires were sent out to 23 countries in March 2005 and replies were received from March 2005 through November 2005. ${ }^{13}$ The questionnaires asked countries, on a measure by measure basis, to respond for each indicator in one of five ways. The response possibilities were as set out in Table 1.

Table 1. Example of questionnaire response categories

\begin{tabular}{|c|c|c|}
\hline \multicolumn{2}{|c|}{ Your response } & \\
\hline \multirow{2}{*}{\begin{tabular}{c} 
Availability of this $\begin{array}{c}\text { indicator in your } \\
\text { country }\end{array}$ \\
\cline { 2 - 3 }
\end{tabular}} & Indicator is currently collected & $\square$ \\
\hline & $\begin{array}{c}\text { Indicator could be constructed from available data } \\
\text { A variant of this indicator could be constructed (Please } \\
\text { describe nature of the variation below) }\end{array}$ \\
\hline & $\begin{array}{c}\text { Data for this indicator might become available in the next } \\
\text { three years }\end{array}$ \\
\hline & Unlikely to become available \\
\hline
\end{tabular}

\section{Analysis steps and criteria for selecting indicators}

30. In assessing whether an indicator met the threshold of 10 or more countries which could supply the data, the Secretariat considered both the number of countries that stated that an indicator was "currently collected" and the number of countries that stated that an indicator "could be constructed from available data".

\section{Summary of the results}

31. The following findings are based on country responses: 5 indicators out of the recommended 86 were "currently collected" in 10 countries or more, 3 in diabetes care (lower extremity amputations rates, annual eye exam and poor $\mathrm{HbAlc}$ control -already included in the initial indicators) and 2 in primary care and prevention (smoking rate and low birth weight rate - with these two already collected by $O E C D$ Health Data). Of the remaining indicators in these two fields and all indicators proposed for cardiac care, mental health care and patient safety, none met the criterion of "currently collected" in 10 countries or more. Eighteen additional indicators could be "constructed from available data" from 10 or more countries. This brought the total number of indicators available to $\mathbf{2 3}$ (none for mental health ${ }^{14}$ ), broken down as follows:

- two for the area of cardiac care (CABG in-hospital mortality rate and PTCA in-hospital mortality)

- $\quad$ three for diabetes care (lower extremity amputations rates, annual eye exam and poor $\mathrm{HbA} 1 \mathrm{c}$ control - already included in the initial indicators data collection, though not fit for international comparisons due to problems in national representativeness of data and other comparability issues)

\footnotetext{
${ }^{13}$ The Slovak Republic only recently joined the HCQI project and therefore did not take part in the survey. The findings will be updated as any additional country information is provided.

${ }^{14}$ Note that for two mental health indicators nine countries stated that the data were available or that they could construct the indicator from available data. These two are: MH7 (hospital readmissions for psychiatric patients) and MH12 (mortality for persons with severe psychiatric disorders).
} 
- $\quad$ nine for patient safety (problems with childbirth, obstetric trauma-vaginal, obstetrics traumacaesarean section, post-operative hip fracture, foreign body left in during procedure, birth trauma-injury to neonate, complications of anaesthesia, transfusion reaction and wrong blood type)

- nine for primary care and prevention (smoking rate, diabetes prevalence, low birth weight rate, obesity prevalence - already collected by OECD Health Data, abortion rates, prevalence of immunisable conditions, physical activity, gonorrhoea/Chlamydia rates and hospitalisation for ambulatory-care sensitive conditions)

32. Based on the above findings, the Expert Group discussed the possibility of adding a small number of these "new" indicators to the 2006 data collection, taking into account both their importance and availability. With the input of the members of the Primary Care and Prevention and Patient Safety panels, five additional indicators were recommended by the HCQI Expert Group for data collection as part of the HCQI 2006 questionnaire: a) asthma admission rate; b) hypertension admission rate; c) diabetes admission rate - these three being preventable causes of hospitalisation (or ambulatory-care sensitive conditions); d) post-operative hip fracture or fall and e) transfusion reaction rate. These indicators were selected for two reasons. First, the 3 indicators of preventable hospitalisations were deemed to be among the most tested of the indicators with broad data availability across countries. Second, patient safety was an area selected as high priority for work in 2006 by the Expert Group, thus the 2006 data collection would allow for an initial testing of the feasibility of international comparisons on two safety indicators using administrative data. When these 5 new indicators were added to the previous 21 , the resulting collection exhausted the indicators that had broad consensus and for which comparable data across countries was available. Table 2 summarises the original collection of 21 indicators and the latest collection of 26 , distinguishing between those that are deemed fit for international comparison and those that are not yet deemed fit on grounds of comparability. 
Table 2. Composition and evolution of the HCQI set of indicators

\begin{tabular}{|c|c|c|}
\hline Indicator & $\begin{array}{l}2003-2005 \text { set of indicators as } \\
\text { published in OECD HWP No. } 22\end{array}$ & 2006 set of indicators \\
\hline Breast cancer five-year survival rate & fit for international comparison & fit for international comparison \\
\hline Mammography screening rate & fit for international comparison & fit for international comparison \\
\hline Cervical cancer five-year survival rate & fit for international comparison & fit for international comparison \\
\hline Cervical cancer screening rate & fit for international comparison & fit for international comparison \\
\hline Colorectal cancer five-year survival rate & fit for international comparison & fit for international comparison \\
\hline $\begin{array}{l}\text { Incidence of vaccine preventable diseases } \\
\text { (Pertussis, measles, and hepatitis B) }\end{array}$ & fit for international comparison & fit for international comparison \\
\hline $\begin{array}{l}\text { Coverage for basic vaccination programme, } \\
\text { age } 2 \\
\text { (Pertussis, measles, and hepatitis B) }\end{array}$ & fit for international comparison & fit for international comparison \\
\hline Asthma mortality rate, ages $5-39$ & fit for international comparison & fit for international comparison \\
\hline $\begin{array}{l}\text { In-hospital mortality rate within } 30 \text { days of } \\
\text { hospital admission for AMI }\end{array}$ & fit for international comparison & fit for international comparison \\
\hline $\begin{array}{l}\text { In-hospital mortality rate within } 30 \text { days of } \\
\text { hospital admission for stroke }\end{array}$ & fit for international comparison & fit for international comparison \\
\hline $\begin{array}{l}\text { In-hospital waiting time for surgery after hip } \\
\text { fracture, over age } 65\end{array}$ & fit for international comparison & fit for international comparison \\
\hline Influenza vaccination, over 65 & fit for international comparison & fit for international comparison \\
\hline Smoking rate & fit for international comparison & fit for international comparison \\
\hline Retinal exam in diabetics & evaluated as not fit & fit for international comparison \\
\hline Asthma admission rate & not reviewed & fit for international comparison \\
\hline Annual HbA1c test for diabetics & evaluated as not fit & evaluated as not fit \\
\hline HbA1c level indicating poor glucose control & evaluated as not fit & evaluated as not fit \\
\hline Major amputations in diabetics & evaluated as not fit & evaluated as not fit \\
\hline Post-operative hip fracture rate & not reviewed & evaluated as not fit \\
\hline Transfusion reaction rate & not reviewed & evaluated as not fit \\
\hline Uncontrolled diabetes admission rate & not reviewed & evaluated as not fit \\
\hline Hypertension admission rate & not reviewed & evaluated as not fit \\
\hline
\end{tabular}

33. The HCQI Expert Group has agreed to conduct developmental work in each of the priority areas, establishing specific expert subgroups for each of them sequentially. Based on the results of the data availability survey and on a review of the clinical and policy importance and scientific soundness ratings given to the indicators, the HCQI Expert Group at its 2005 meeting recommended that the initial focus areas would be patient safety and mental health. Both subgroups were formed in April 2006 and important progress in addressing methodological issues has been and continues to be made. 


\section{DATA COMPARABILITY ISSUES}

34. In OECD Health Working Paper No. 22 on the Initial Indicators for the HCQI Project, an extensive examination of a range of data comparability issues was reported. Data comparability issues persist in the current indicator set requiring further investigation. These issues were discussed at the recent HCQI Experts Meeting in October 2006. These issues include:

- the use of non-nationally representative data

- $\quad$ presentation of administrative versus survey data for cancer screening

- harmonising data recall periods for cancer survival and screening

- the use of a truncated standard population for age adjustment

35. The use of non-nationally representative data In Health Working Paper No. 22, the OECD Secretariat published data from countries that was not strictly nationally representative. This was an explicit recommendation of the HCQI Expert Group in 2004, when several countries stated that they were unable to provide nationally representative data for cancer survival. At the time, the HCQI Expert Group discussed this and recommended that non-nationally representative data should be included where countries provided a written statement that the data was broadly generalisable to the nation as a whole. The HCQI Expert Group reviewed this decision in October 16-17, 2006 and recommended that non-nationally representative data should be presented separately in the working paper, noting the reason why the data was presented in a separate note. It was hoped that this solution would avoid confusing comparisons between nationally representative and non-nationally representative data.

36. Presentation of administrative versus survey data for cancer screening In Health Working Paper No. 22, data were presented on cancer screening for breast and cervical cancer. In many cases, these data came from varying sources across countries, namely administrative and programmatic data or household surveys. In the analysis reported in Health Working Paper No. 22, there appeared to be a systematic difference between these two data sources, in that administrative records often provided lower estimates than survey data. The most recent round of data collection in 2006 shows that there no longer appears to be a systematic difference between these two data sources (see Figure 1 below.) However, the broader literature continues to report differences between administrative records and surveys. 
Figure 1. Findings on differences between administrative and survey data for cancer screening

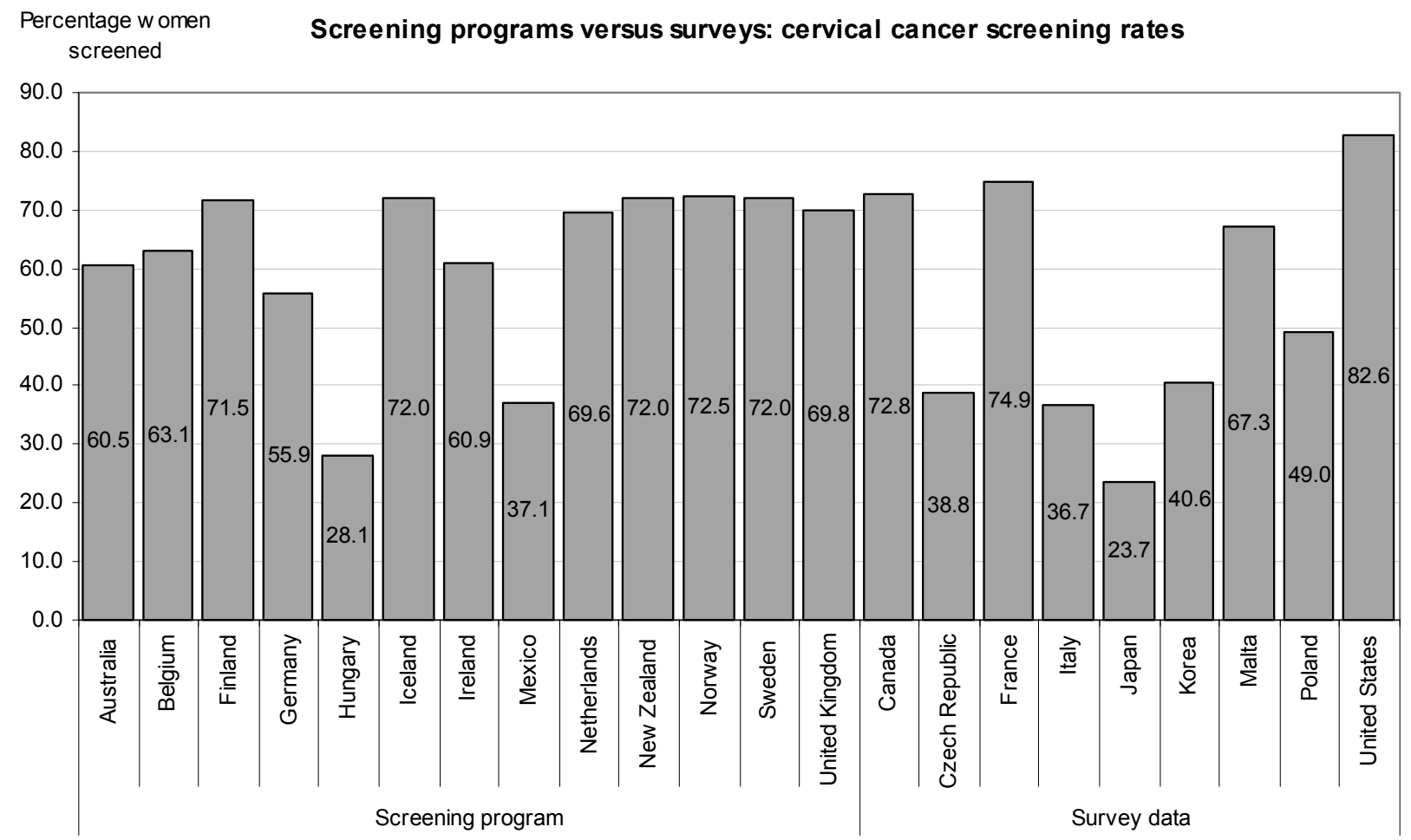

37. Harmonising data recall periods for cancer survival and screening An additional issue with the cancer screening and cancer survival data is that recall periods differ widely across countries. In the area of cancer survival, countries also have widely varying time periods from which they are presenting data, since many countries do not compile their cancer survival statistics annually, but rather every 3 or 5 years, depending on the periodicity of the data system. Data will be reported according to the most recent data available in the survival interval in order to simplify data presentation. The Secretariat will further work with countries to attempt to harmonise these data year differences, using the EUROCARE ${ }^{15}$ data as one guide since EUROCARE countries have already attempted to agree on data years. For cancer screening, many countries have reported data simply according to national survey specifications. That is, if the question on the national survey and the national report for cancer screening is "How often in the last year did you receive a screening for cervical cancer?" then this was the data reported, despite the fact that this does not correspond to the indicator specification ${ }^{16}$.

15 The EUROCARE project (European cancer registries study on cancer patients' survival and care) is an international collaborative study on the survival of cancer patients in Europe. It currently involves 67 populationbased cancer registries operating in 22 European countries. EUROCARE has been promoted by the European Community.

${ }^{16}$ The indicator specification for the numerator for breast cancer is "Number of women 50-69 reporting of having received a bilateral mammography within the past year or number of women 50-69 screened through an organised program within the past year." The indicator specification for the numerator for cervical cancer screening is "Number of women 20-69 reporting cervical cancer screening within the past 3 years or number of women aged 20-69 screened through an organised program within the past 3 years". 


\section{DELSA/HEA/WD/HWP(2007)4}

38. In October 2006, the HCQI Experts agreed that countries should try to harmonise these data over the next year so that the 2008 data collection would represent more comparable data. It was agreed, however, that the data should be presented as collected, with appropriate notes on differing recall and data years.

39. The use of a truncated standard population for age adjustment Age standardisation is necessary since a country's age structure, depending on the nature of the disease and the structure of the population, can influence the international comparison of health system performance. For example, if Country A's population is notably older as a whole than Country B's population, we would expect there to be higher rates of chronic diseases and thus the population, as a whole, may appear "sicker." This may cause apparent differences in performance for diseases whose incidence and prognosis depends on the age at diagnosis. Such apparent differences are not within the control of the health system, and adjustments for them should be made when comparing performance levels on quality indicators across countries. The same holds true for longitudinal comparisons within one country if the country's age structure changes significantly over time.

40. To account for such differences in age structure, age adjustments are made based on standardised populations. The resulting age-adjusted rates reflect a country's hypothetical performance on a standard population and should thus be viewed as relative indexes rather than actual measures. These adjustments can become extremely important when examining data over time and comparing performance across geographical areas. Many national reports on quality of care use some form of age adjustment to account for changes over time in the age structure of the population (CIHI, 2004).

41. An analysis of the impact of age standardisation was undertaken as part of OECD Health Working Paper No. 22. The findings indicated that there appeared to be virtually no difference in countries' relative rankings using either a 1980 or 2005 OECD standard population in calculating cancer survival rates. Secondly, there appeared to be only small differences in the ranking of countries between the use of a 1980 OECD standard population and the disease specific EUROCARE cancer population for relative survival rates. There was, however, some modest influence in the ranking of countries between the use of the 1980 OECD standard population and the EUROCARE cancer population in terms of observed survival. Relative survival rates are the ratio of the disease-specific mortality to overall mortality in a given population. The above findings, therefore, are not surprising as these relative survival rates control to some degree for differences in the age structure of the general population. Lastly, it appears that the use of a truncated version of the 1980 OECD standard population (at age 45+, thus shaping the age structure more closely to a disease specific population) provides estimates moderately different from those based on the EUROCARE cancer population, although the differences were more important for some types of cancers (cervical cancer) than for others. The recommendation at that point was to keep the 1980 OECD standard population as reference for standardisation in 2006 data collection and start exploring more specialised adjustment approaches for both relative and observed survival rates.

42. Working Paper No. 22 introduced also a recommendation to consider the need to apply age standardisation to the calculation of case fatality rates. So far, the Secretariat has suggested to countries to provide age standardised data or specific age groups rates for the two case fatality rate indicators, however, it has not been set as a requisite to accept data in any of the data collections hitherto due to the inability of several countries to provide data with this level of disaggregation. However the potential impact of this lack of age standardisation is not minor. In a presentation to the HCQI Experts' Group in October 2006, Dr. Max Köster, one of Sweden's representatives in the Expert Group, presented findings from an analysis examining this issue for AMI and stroke survival rates. This analysis looked at Swedish data and compared different adjustments by age and discussed whether all ages should be included. He showed how $98 \%$ of the deaths for ischemic stroke occurred in age groups of 45+. However, in the 1980 OECD standard population that was used in the HCQI Initial Indicators Report, only $31 \%$ of the population is found in this age group. This means that variations in small numbers of deaths in the younger age groups can have a 
major impact if the standard population for age adjustment differs sharply in structure from the age pattern of mortality. The analysis presented two different scenarios for the same data; in Scenario A none of the 8 cases presented among the population 10-14 years old dies in the 30 days following diagnosis. In scenario $\mathrm{B}$ one of the cases ends fatally. The specific fatality rate for this group would actually rise from 0 to $12.5 \%$, and due to the weight attached to this segment of population in 1980 OECD population the corresponding standardised rate will increase from $3.66 \%$ in scenario A to $4.70 \%$ in B. (Table 2).

Table 3. 2004 Admission based ischemic stroke case fatality in Sweden

\begin{tabular}{|c|c|c|c|c|c|c|c|c|}
\hline Ages & $\begin{array}{l}\text { Number of } \\
\text { admissions }\end{array}$ & $\begin{array}{c}\text { "True" } \\
\text { weights } \\
\text { Incidence } \\
\text { distribution }\end{array}$ & $\begin{array}{c}\text { Accum } \\
\text { "true" } \\
\text { weight } \\
(\%)\end{array}$ & $\begin{array}{c}\text { Fatality } \\
\text { rate } \\
\text { scenario } \\
A^{17}\end{array}$ & $\begin{array}{l}\text { Fatality } \\
\text { rate } \\
\text { scenario } \\
\text { B }^{18}\end{array}$ & $\begin{array}{c}\text { Weights } \\
1980\end{array}$ & $\begin{array}{c}\text { Accum } \\
1980 \\
\text { weight } \\
(\%)\end{array}$ & $\begin{array}{c}\text { Rates } \\
\text { standardised } \\
\text { to OECD } 1980\end{array}$ \\
\hline \multirow{2}{*}{ TOTAL } & \multirow{2}{*}{35323} & \multirow{2}{*}{$100,00 \%$} & \multirow{11}{*}{1.81} & \multirow{2}{*}{$9,18 \%$} & \multirow{2}{*}{$9,18 \%$} & \multirow{2}{*}{100,00} & \multirow{11}{*}{68.67} & Scenario A \\
\hline & & & & & & & & $3.66 \%$ \\
\hline $0-4$ & 32 & $0,09 \%$ & & $12,50 \%$ & $12,50 \%$ & 7,94 & & Scenario B \\
\hline 5- 9 & 6 & $0,02 \%$ & & $0,00 \%$ & $0,00 \%$ & 8,09 & & $4.70 \%$ \\
\hline $10-14$ & 8 & $0,02 \%$ & & $0,00 \%$ & $12,50 \%$ & 8,30 & & \\
\hline $15-19$ & 13 & $0,04 \%$ & & $0,00 \%$ & $0,00 \%$ & 8,56 & & \\
\hline $20-24$ & 36 & $0,10 \%$ & & $5,56 \%$ & $5,56 \%$ & 8,20 & & \\
\hline $25-29$ & 53 & $0,15 \%$ & & $1,89 \%$ & $1,89 \%$ & 7,81 & & \\
\hline $30-34$ & 73 & $0,21 \%$ & & $6,85 \%$ & $6,85 \%$ & 7,63 & & \\
\hline $35-39$ & 155 & $0,44 \%$ & & $0,65 \%$ & $0,65 \%$ & 6,31 & & \\
\hline $40-44$ & 256 & $0,72 \%$ & & $2,34 \%$ & $2,34 \%$ & 5,83 & & \\
\hline $45-49$ & 422 & $1,19 \%$ & \multirow{9}{*}{98.19} & $2,37 \%$ & $2,37 \%$ & 5,56 & \multirow{9}{*}{31.31} & \\
\hline $50-54$ & 736 & $2,08 \%$ & & $2,45 \%$ & $2,45 \%$ & 5,46 & & \\
\hline $55-59$ & 1612 & $4,56 \%$ & & $3,41 \%$ & $3,41 \%$ & 5,08 & & \\
\hline $60-64$ & 2416 & $6,84 \%$ & & $2,90 \%$ & $2,90 \%$ & 3,89 & & \\
\hline $65-69$ & 3045 & $8,62 \%$ & & $4,47 \%$ & $4,47 \%$ & 3,88 & & \\
\hline $70-74$ & 4312 & $12,21 \%$ & & $6,52 \%$ & $6,52 \%$ & 3,18 & & \\
\hline $75-79$ & 6292 & $17,81 \%$ & & $7,69 \%$ & $7,69 \%$ & 2,26 & & \\
\hline $80-84$ & 7649 & $21,65 \%$ & & $10,52 \%$ & $10,52 \%$ & 1,23 & & \\
\hline 85- & 8207 & $23,23 \%$ & & $16,61 \%$ & $16,61 \%$ & 0,77 & & \\
\hline
\end{tabular}

The recommendation issued by the Expert Group is that, for the OECD's HCQI 2008 data collection, a new OECD standard population, truncated at ages below 45, should be developed, tested and disseminated to participating countries for age adjustment of survival and mortality rates. This will serve as a better approximation to a disease-specific population. The Secretariat has developed an updated standard OECD reference population for 2005, to account for the changes in age structure across OECD countries in the last decades. This population will serve as the basis for the proposed truncation exercise.

\footnotetext{
${ }^{17} 0$ cases resulting in death in the group $10-14$

${ }^{18} 1$ case resulting in death in the group 10-14
} 


\section{"INITIAL" (2003-2005) INDICATORS: 2006 SPECIFICATIONS AND DATA RESULTS}

43. This section of the report presents data updated in 2006 for the 2003-2005 set of indicators. The indicators listed in this section are the "initial" HCQI indicators published in OECD Health Working Paper No. 22. They are listed in column 1 of Table 2 above. A full discussion of the scientific soundness and clinical importance of the indicators presented in this section is presented in OECD Health Working Paper No. 22 (http://www.oecd.org/dataoecd/1/34/36262514.pdf) and is not repeated here.

44. As mentioned earlier, it is clear that while the indicators listed in this section have met the HCQI criteria for being scientifically sound, clinically important and having comparable data across countries, this does not mean that they are free of data comparability issues. This paper reports fully on the data comparability for all indicators. The OECD Secretariat will continue to work with countries to improve the comparability of the indicators listed in this section.

45. The following table summarises the data availability for all the indicators presented in this section of the report. 
Table 4. Availability of data for 'Initial' (2003-2005) Indicators presented in this section

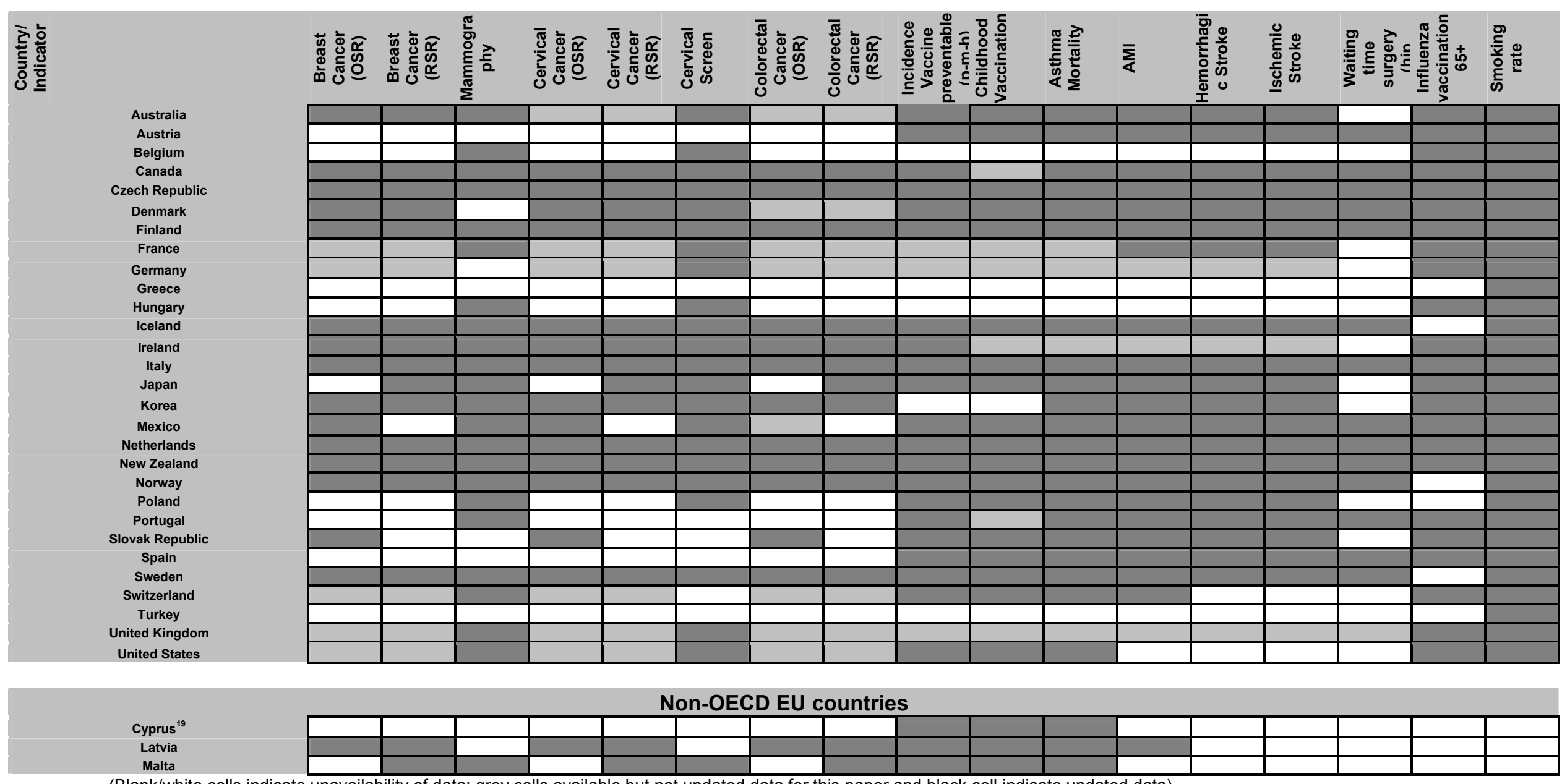

(Blank/white cells indicate unavailability of data; grey cells available but not updated data for this paper and black cell indicate updated data)

\footnotetext{
${ }^{19}$ See footnotes 2 and 3 on page 3 .
} 
DELSA/HEA/WD/HWP(2007)4

\section{Breast cancer five-year survival rate}

Operational Definition

A. 5-year observed survival rate (OSR), breast cancer (Diagnostic code: ICD-9 C:174.xx, ICD 10: C50.x) diagnosis.

Numerator: Number of women diagnosed with breast cancer surviving five years after

Denominator: Number of women diagnosed with breast cancer.

B. 5-year relative survival rate (RSR), breast cancer (Diagnostic code: ICD-9 C:174.xx, ICD 10: C50.x)

Numerator: Observed rate of women diagnosed with breast cancer surviving five years after diagnosis.

Denominator: Expected survival rate of a comparable group from the general population. 
Table 5. Breast cancer five-year survival rate

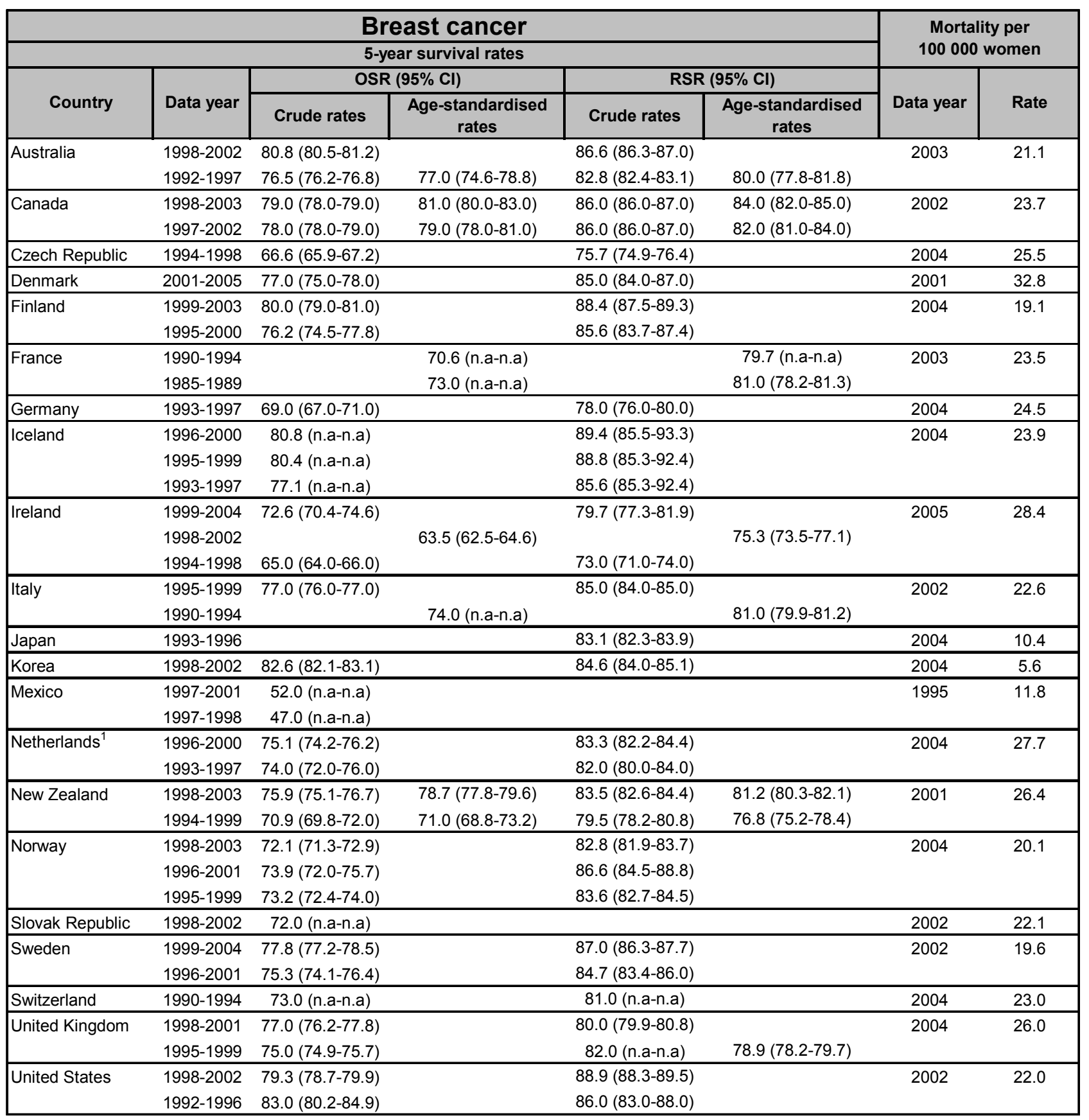




\begin{tabular}{|c|c|c|c|c|c|c|c|}
\hline \multicolumn{6}{|c|}{ Non-OECD EU countries } & \multicolumn{2}{|c|}{$\begin{array}{c}\text { Mortality per } 100000 \\
\text { women }\end{array}$} \\
\hline \multirow[b]{2}{*}{ Country } & \multirow[b]{2}{*}{ Data year } & \multicolumn{2}{|c|}{ OSR $(95 \% \mathrm{Cl})$} & \multicolumn{2}{|c|}{$\operatorname{RSR}(95 \% \mathrm{Cl})$} & \multirow[b]{2}{*}{ Data year } & \multirow[b]{2}{*}{ Rate } \\
\hline & & Crude rates & $\begin{array}{l}\text { Age-standardised } \\
\text { rates }\end{array}$ & Crude rates & $\begin{array}{l}\text { Age-standardised } \\
\text { rates }\end{array}$ & & \\
\hline Latvia & $1999-2003$ & 59.5 (n.a-n.a) & & 66.6 (n.a-n.a) & & 2005 & 32.5 \\
\hline \multirow[t]{2}{*}{ Malta } & 1998-2002 & & & $78.0(73.0-82.0)$ & & 2005 & 28.1 \\
\hline & $1993-1994$ & & & & $74.8(69.0-81.0)$ & & \\
\hline
\end{tabular}

Notes:

Reference population for age-standardised rates: OECD 1980, except for France, Ireland, Italy and Malta who refer to the Eurocare-3 population and the Slovak Republic to the Standard European population.

$\mathrm{Cl}$ stands for "Confidence Interval". Observed survival rates are an estimation of the probability of a patient having survived five years after being diagnosed of cancer based on the actual data available. Relative survival rates adjusts this probability of surviving a cancer diagnosis by the general probability of surviving attributable to any member of the same age group independently of whether they do suffer cancer Thus the $95 \%$ confidence interval $(\mathrm{Cl})$ illustrates the degree of variability associated with these estimates. Wide confidence intervals indicate high variability, therefore, these estimates should be interpreted with due caution. When estimates are based on a small number of cases, it is more likely that observed differences are due to random, rather than systematic influences.

"n.a" stands for "no data available".

1. Data is not national, but has been weighted by the incidence they represent upon the nation. See Sources and Methods for more information.

Source: HCQI Project, 2007. Mortality data: OECD Health Data 2007, July 07 
DELSA/HEA/WD/HWP(2007)4

Breast cancer five-year survival rate, sources and methods

\begin{tabular}{|c|c|c|c|c|c|c|c|}
\hline Country & Source & $\begin{array}{l}\text { Diagnosis } \\
\text { Code }\end{array}$ & $\begin{array}{c}\text { Age } \\
\text { groups } \\
\text { included }\end{array}$ & $\begin{array}{c}\text { Additional } \\
\text { years }\end{array}$ & $\begin{array}{l}\text { Is this a national } \\
\text { registry? }\end{array}$ & $\begin{array}{l}\text { Reference } \\
\text { population }\end{array}$ & Comments \\
\hline Australia & $\begin{array}{l}\text { Australian Institute for Health and Welfare \& } \\
\text { National Breast Cancer Centre 2006. Breast } \\
\text { Cancer in Australia: an overview, 2006. AlHW } \\
\text { cat. no. CAN 29. Canberra: AlHW. }\end{array}$ & & $20+$ & & & $\begin{array}{l}1980 \mathrm{OECD} \\
\text { population }\end{array}$ & \\
\hline Canada & Canadian Cancer Registry & $\begin{array}{l}\text { Canadian } \\
\text { data coded } \\
\text { using ICDO- } \\
3 \text { (not ICD } \\
9 / 10)\end{array}$ & $15-99$ & & Yes & $\begin{array}{l}1980 \mathrm{OECD} \\
\text { population }\end{array}$ & $\begin{array}{l}\text { Missing cases treated as: we rely on } \\
\text { record linkage for vital status (i.e. passive } \\
\text { follow-up) and as such we cannot identify } \\
\text { cases lost to follow-up. So we don't } \\
\text { "handle" them in any way. Practically } \\
\text { speaking there will be people who die } \\
\text { outside of the country and whose death is } \\
\text { not recorded in the national mortality } \\
\text { database. }\end{array}$ \\
\hline $\begin{array}{l}\text { Czech } \\
\text { Republic }\end{array}$ & $\begin{array}{l}\text { National Cancer Registry of the Czech } \\
\text { Republic; Vital Statistics (Czech Statistical } \\
\text { Office) }\end{array}$ & & & 1980-1998 & Yes & & Missing cases treated as: survivors \\
\hline Denmark & National Cancer Registry & ICD-10 C50 & & From 1977 & Yes & & $\begin{array}{l}\text { Missing cases treated as: dropped out. } \\
\text { The calculations are based on unique } \\
\text { identifiers, why few people are lost to } \\
\text { follow-up. The Update is based on the } \\
\text { National Hospital Register for patients } \\
\text { diagnosed in } 2001 \text { and reported in } 2005 \text {. }\end{array}$ \\
\hline Finland & Finnish Cancer Register & ICD-10 C50 & & $1953-2004$ & Yes & & $\begin{array}{l}\text { Missing cases treated as: No losses to } \\
\text { follow-up }\end{array}$ \\
\hline
\end{tabular}


DELSA/HEA/WD/HWP(2007)4

\begin{tabular}{|c|c|c|c|c|c|c|c|}
\hline Country & Source & $\begin{array}{c}\text { Diagnosis } \\
\text { Code }\end{array}$ & $\begin{array}{c}\text { Age } \\
\text { groups } \\
\text { included }\end{array}$ & $\begin{array}{c}\text { Additional } \\
\text { years }\end{array}$ & $\begin{array}{l}\text { Is this a national } \\
\text { registry? }\end{array}$ & $\begin{array}{l}\text { Reference } \\
\text { population }\end{array}$ & Comments \\
\hline France & $\begin{array}{l}\text { EUROCARE-3: Electronic availability of } \\
\text { EUROCARE } 3 \text { data: a tool for further analysis P } \\
\text { Roazzi, Annals of Oncology } 14150-155,2003 \text {. } \\
\text { National death causes database/Available } \\
\text { Eurostat database. }\end{array}$ & ICD-10 C50 & $15+$ & & $\begin{array}{l}4 \text { regional registries: } \\
\text { Calvados, Côte d'Or, } \\
\text { Isère, Bas-Rhin } \\
\text { representing } \\
3.199 .575 \text { persons, } \\
\text { i.e. } 5.6 \% \text { of French } \\
\text { population in } 1990 \text {. } \\
\text { Generalisable. }\end{array}$ & & ICSS population \\
\hline Germany & Saarland Cancer Registry & ICD-9 174 & $15-89$ & & $\begin{array}{l}\text { State of Saarland. } \\
\text { Not generalisable }\end{array}$ & & $\begin{array}{l}\text { Includes only: Saarland residents, } 15 \text { and } \\
89 \text { yrs at diagnosis, with } \\
\text { invasive/malignant cases, first primaries. } \\
\text { Saarland residents at time of diagnosis, } \\
\text { age at time of diagnosis between ages } \\
\text { 15-89. Date of diagnosis } 1993-1997 . \\
\text { Follow-up to December } 2000 .\end{array}$ \\
\hline Iceland & Icelandic Cancer Registry & ICD-10 C50 & & $1955-1995$ & Yes & & Missing cases treated as: non-survivors \\
\hline Ireland & NCRI. Irish National Cancer Registry & ICD-10 C50 & $15-99$ & & & $\begin{array}{l}\text { Eurocare 3 } \\
\text { standard } \\
\text { patient } \\
\text { populations }\end{array}$ & $\begin{array}{l}\text { Missing cases treated as: Follow up is } \\
\text { passive, by means of matching registered } \\
\text { cases to death certificates. Currently } \\
\text { matching completed to } 31 \text { December } \\
2003 \text { (censoring date) and patients not } \\
\text { matched by this date (known to have died } \\
\text { by this date) are assumed to be alive }\end{array}$ \\
\hline Italy & Registro Nazionale Tumori & ICD-10 C50 & $14+$ & & Yes & & Missing cases treated as: dropped out \\
\hline Japan & $\begin{array}{l}\text { Tsukuma H, Ajiki W, loka A, Oshima A, and } \\
\text { Research Group of Population-Based Cancer } \\
\text { Registry of Japan, Survival of cancer patients } \\
\text { diagnosed in 1993-96: collaborative study of } \\
\text { population-based cancer registries in Japan, } \\
\text { Japanese Journal of Clinical Oncology, 36: 602- } \\
607,2006\end{array}$ & ICD-9 174 & & & & & \\
\hline
\end{tabular}


DELSA/HEA/WD/HWP(2007)4

\begin{tabular}{|l|l|l|l|l|l|l|}
\hline Country & \multicolumn{1}{|c|}{ Source } & $\begin{array}{c}\text { Diagnosis } \\
\text { Code }\end{array}$ & $\begin{array}{c}\text { Age } \\
\text { groups } \\
\text { included }\end{array}$ & $\begin{array}{l}\text { Additional } \\
\text { years }\end{array}$ & $\begin{array}{l}\text { Is this a national } \\
\text { registry? }\end{array}$ & $\begin{array}{l}\text { Reference } \\
\text { population }\end{array}$ \\
\hline Korea & $\begin{array}{l}\text { Korea Central Cancer Registry Source of } \\
\text { mortality data: Annual Report on the Cause of } \\
\text { Death Statistics. Korea National Statistical } \\
\text { Office, 2006. }\end{array}$ & ICD-10 C50 & & & Yes & $\begin{array}{l}\text { Comments } \\
\text { The Korea Central Cancer Registry has } \\
\text { been collaborating with the Korean Breast } \\
\text { Cancer Society's breast cancer registry to } \\
\text { produce national breast cancer statistics. } \\
\text { 2. Since our cancer incidence DB is under } \\
\text { review of the International Agency for } \\
\text { Research on Cancer (IARC), statistical } \\
\text { figures may change after the completion } \\
\text { of the review. }\end{array}$ \\
\hline Mexico & $\begin{array}{l}\text { Estadistica de Casos de Cancer de Mama: } \\
\text { Servicio de Oncologia Mamaria Hospital de } \\
\text { Ginecobstetricia No. 4 Luis Castelazo Ayala. } \\
\text { Source of mortality data: Sistema Institucional } \\
\text { de Mortalidad (SISMOR). }\end{array}$ & ICD-10 C50 & & & $\begin{array}{l}\text { No. By size it's } \\
\text { generalisable to } \\
\text { national level. This } \\
\text { Hospital covered 22\% } \\
\text { of total cases of } \\
\text { breast cancer in the } \\
\text { country }\end{array}$ & \\
\hline
\end{tabular}


DELSA/HEA/WD/HWP(2007)4

\begin{tabular}{|c|c|c|c|c|c|c|c|}
\hline Country & Source & $\begin{array}{c}\text { Diagnosis } \\
\text { Code }\end{array}$ & $\begin{array}{l}\text { Age } \\
\text { groups } \\
\text { included }\end{array}$ & $\begin{array}{c}\text { Additional } \\
\text { years }\end{array}$ & $\begin{array}{l}\text { Is this a national } \\
\text { registry? }\end{array}$ & $\begin{array}{l}\text { Reference } \\
\text { population }\end{array}$ & Comments \\
\hline Netherlands & $\begin{array}{l}\text { National Institute of Public Health and the } \\
\text { Environment. Source of Mortality: Statline. } \\
\text { Voorbrug: Statistics Netherlands, } 2006 \text { Website } \\
\text { http://statline.cbs.nl/StatWeb/Start.asp? ?p=Sear } \\
\text { ch/Search\&LA=EN\&DM=SLEN. }\end{array}$ & ICD-10 C50 & All & & $\begin{array}{l}\text { No. In the } \\
\text { Netherlands, the } \\
\text { National Cancer } \\
\text { Registry of the } \\
\text { Association of } \\
\text { Comprehensive } \\
\text { Cancer Centres } \\
\text { (CCC) delivers } \\
\text { incidence rates. } \\
\text { However, survival } \\
\text { rates can not be } \\
\text { calculated for the } \\
\text { whole country. At this } \\
\text { moment, three } \\
\text { Comprehensive } \\
\text { Cancer Centres have } \\
\text { data on cancer } \\
\text { survival. In a few } \\
\text { years, all CCC's will } \\
\text { deliver data on } \\
\text { cancer survival. Data } \\
\text { therefore cover over } \\
40 \% \text { of Dutch } \\
\text { population. Data have } \\
\text { been weighted by the } \\
\text { incidence of the CCC } \\
\text { into the total } \\
\text { incidence of the } \\
\text { country. }\end{array}$ & & $\begin{array}{l}\text { Missing cases treated as: the CCC's } \\
\text { obtain data of persons in the population } \\
\text { who died. These data are obtained from } \\
\text { the Municipal Basis Registry, which is to a } \\
\text { large extent complete. Patients, of whom } \\
\text { no death report is obtained, are still alive. } \\
\text { Emigrants are censored. Overall, almost } \\
100 \% \text { of follow-up is complete. }\end{array}$ \\
\hline $\begin{array}{l}\text { New } \\
\text { Zealand }\end{array}$ & $\begin{array}{l}\text { New Zealand Cancer Registry (NZCR). Source } \\
\text { of mortality data: NZHIS Mortality data } \\
\text { collection }\end{array}$ & $\begin{array}{l}\text { ICD-10-AM } \\
\text { (Australian } \\
\text { Modification } \\
\text { ) C50 }\end{array}$ & & $1994-2006$ & Yes & $\begin{array}{l}\text { OECD } \\
\text { standard } \\
\text { population } \\
1980 \text { (only for } \\
\text { relative rates) }\end{array}$ & $\begin{array}{l}\text { Missing cases treated as: passive follow- } \\
\text { up, no losses DCO (Death certificate } \\
\text { only) cases were excluded }\end{array}$ \\
\hline Norway & Cancer Registry of Norway & ICD-10 C50 & & & & & Missing cases treated as: dropped out \\
\hline
\end{tabular}


DELSA/HEA/WD/HWP(2007)4

\begin{tabular}{|c|c|c|c|c|c|c|c|}
\hline Country & Source & $\begin{array}{l}\text { Diagnosis } \\
\text { Code }\end{array}$ & $\begin{array}{l}\text { Age } \\
\text { groups } \\
\text { included }\end{array}$ & $\begin{array}{l}\text { Additional } \\
\text { years }\end{array}$ & $\begin{array}{l}\text { Is this a national } \\
\text { registry? }\end{array}$ & $\begin{array}{l}\text { Reference } \\
\text { population }\end{array}$ & Comments \\
\hline $\begin{array}{l}\text { Slovak } \\
\text { Republic }\end{array}$ & National cancer registry & ICD-10 C50 & & & Yes & $\begin{array}{l}\text { standard } \\
\text { European } \\
\text { population }\end{array}$ & $\begin{array}{l}\text { Missing cases treated as: returned to the } \\
\text { reporting units for correction, therefore we } \\
\text { do not have missing cases. }\end{array}$ \\
\hline Sweden & The Swedish Cancer Register & ICD-9 174 & & $1960-1998$ & yes & & $\begin{array}{l}\text { Missing cases treated as: censored in a } \\
\text { survival analyses (actuarial method) }\end{array}$ \\
\hline \multirow[t]{2}{*}{$\begin{array}{l}\text { United } \\
\text { Kingdom }\end{array}$} & \multirow[t]{2}{*}{$\begin{array}{l}\text { Office for National Statistics/Department of } \\
\text { Health }\end{array}$} & \multirow[t]{2}{*}{ ICD-9 174} & \multirow[t]{2}{*}{$15-99$} & & \multirow[t]{2}{*}{ Data are for England } & \multirow[t]{2}{*}{ OECD 1980} & $\begin{array}{l}\text { The age profile of the England and Wales } \\
\text { cancer population differs from the OECD } \\
\text { population. }\end{array}$ \\
\hline & & & & & & & $\begin{array}{l}\text { For instance, } 51 \% \text { of the adult population } \\
\text { is age } 15-39, \text { but only } 6.1 \% \text { of breast } \\
\text { cancer cases are in this age group. }\end{array}$ \\
\hline $\begin{array}{l}\text { United } \\
\text { States }\end{array}$ & $\begin{array}{l}\text { Surveillance Epidemiology and End Results } \\
\text { (SEER) Program (www.seer.cancer.gov) } \\
\text { SEER*Stat Database: Incidence - SEER 9 } \\
\text { Regs Public-Use, Nov } 2004 \text { Sub (1973-2002), } \\
\text { National Cancer Institute, DCCPS, Surveillance } \\
\text { Research Program, Cancer Statistics Branch, } \\
\text { released April 1, 2005, based on the November } \\
2004 \text { submission }\end{array}$ & & & & & OECD 1980 & \\
\hline
\end{tabular}

\begin{tabular}{|c|c|c|c|c|c|c|c|}
\hline \multicolumn{8}{|c|}{ Non-OECD EU countries } \\
\hline Country & Source & $\begin{array}{l}\text { Diagnosis } \\
\text { Code }\end{array}$ & $\begin{array}{c}\text { Age } \\
\text { groups } \\
\text { included }\end{array}$ & $\begin{array}{c}\text { Additional } \\
\text { years }\end{array}$ & $\begin{array}{l}\text { Is this a national } \\
\text { registry? }\end{array}$ & $\begin{array}{l}\text { Reference } \\
\text { population }\end{array}$ & Comments \\
\hline Latvia & $\begin{array}{l}\text { Cancer Registry. Source of mortality data: } \\
\text { National Death Causes Database. }\end{array}$ & ICD-10 C50 & & & Yes & & $\begin{array}{l}\text { Missing cases treated as: In the } \\
\text { calculation from "missing cases" } 1 / 2 \\
\text { treated as survivors and other } 1 / 2 \text { as non- } \\
\text { survivors }\end{array}$ \\
\hline
\end{tabular}


DELSA/HEA/WD/HWP(2007)4

\begin{tabular}{|c|c|c|c|c|c|c|c|}
\hline \multicolumn{8}{|c|}{ Non-OECD EU countries } \\
\hline Country & Source & $\begin{array}{l}\text { Diagnosis } \\
\text { Code }\end{array}$ & $\begin{array}{c}\text { Age } \\
\text { groups } \\
\text { included }\end{array}$ & $\begin{array}{c}\text { Additional } \\
\text { years }\end{array}$ & $\begin{array}{l}\text { Is this a national } \\
\text { registry? }\end{array}$ & $\begin{array}{l}\text { Reference } \\
\text { population }\end{array}$ & Comments \\
\hline Malta & $\begin{array}{l}\text { Malta National Cancer Registry: } \\
\text { http://www.sahha.gov.mt/pages.aspx?page=91 } \\
\text { Source of mortality data: WHO-HFA: } \\
\text { http://data.euro.who.int/hfadb/ }\end{array}$ & & & & & & $\begin{array}{l}\text { Mortality figures are age-standardised } \\
\text { using European Standard Population }\end{array}$ \\
\hline
\end{tabular}




\section{Mammography screening rate}

\section{Operational Definition}

Numerator: Number of women ages 50-69 reporting having received a bilateral mammography within the past year.

Denominator: Number of women ages 50-69 answering survey questions on mammography or eligible for organised screening programme.

Table 6. Mammography screening rate

\begin{tabular}{|c|c|c|c|c|c|c|c|c|}
\hline \multirow{2}{*}{ Country } & \multicolumn{7}{|c|}{$\%$ women $50-69$ screened } & \multirow{2}{*}{ Survey or screening } \\
\hline & 2000 & 2001 & 2002 & 2003 & 2004 & 2005 & 2006 & \\
\hline Australia & 56.9 & 57.1 & 56.1 & 55.6 & & & & screening program \\
\hline Belgium & 38 & 50 & 50 & 56 & 56 & & & screening program \\
\hline Finland & & 88 & & 87.7 & & & & screening program \\
\hline Hungary $^{1}$ & 26.7 & 28.9 & 48.3 & 60.2 & 55.1 & & & screening program \\
\hline Iceland $^{2}$ & 61 & 62 & 62 & 62 & 61 & 61 & & screening program \\
\hline Ireland $^{3}$ & & & 78.4 & 79.5 & 77.1 & 76.6 & & screening program \\
\hline Japan & 1.2 & 1.7 & 2.1 & 2.6 & 4.1 & & & screening program \\
\hline Netherlands & 78.7 & 78.8 & 78.8 & 80.4 & 81.7 & 81.9 & & screening program \\
\hline New Zealand ${ }^{3}$ & & & 63 & & & & & screening program \\
\hline Norway & & & & 98 & & & & screening program \\
\hline Portugal & & & & 60.1 & & & & screening program \\
\hline Sweden $^{5}$ & & & & & 83.6 & & & screening program \\
\hline United Kingdom ${ }^{\prime}$ & 69.3 & 70.2 & 69.8 & 69.3 & 69 & 69.5 & & screening program \\
\hline Canada & 69.5 & & & 70.6 & & 70.4 & & survey \\
\hline Czech Republic & & & 26.6 & & & & & survey \\
\hline France & & & 55 & 72.8 & & & & survey \\
\hline Italy ${ }^{4}$ & 29 & & & & 59 & & & survey \\
\hline Korea & & & & & & 33.6 & & survey \\
\hline Mexico & & & & & & 22.1 & 63.5 & survey \\
\hline Poland & & & & & 15.4 & & & survey \\
\hline Slovak Republic & 6.9 & 9.1 & 11.4 & 12.4 & 14.8 & 17.1 & & Survey \\
\hline Switzerland & & & 27 & & & & & survey \\
\hline United States & 62.2 & & & 60.8 & & & & survey \\
\hline
\end{tabular}

\begin{tabular}{|c|c|c|c|}
\hline \multicolumn{4}{|c|}{ Non-OECD EU countries } \\
\hline Country & Year & $\%$ & Survey or screening \\
\hline Malta $^{2}$ & 2002 & 57.3 & survey \\
\hline
\end{tabular}

Notes:

1. $45-65$ years.

2. 40-69 years.

3. $50-64$ years.

4. $55-69$ years.

5. 50-74 years.

Source: HCQI Project, 2007

\section{Mammography screening rate, sources and methods}

http://www.ecosante.org/OCDEENG/370010.html 
DELSA/HEA/WD/HWP(2007)4

\section{Cervical cancer five-year survival rate}

Operational Definition

A. 5-year observed survival rate (OSR), cervical cancer (Diagnostic code: ICD-9 C:180.xx; ICD-10: C53.x)

Numerator: Number of women diagnosed with cervical cancer surviving five years after diagnosis.

Denominator: Number of women diagnosed with cervical cancer.

B. 5-year relative survival rate (RSR), cervical cancer (Diagnostic code: ICD-9 C:180.xx; ICD-10: C53.x)

Numerator: Observed rate of women diagnosed with cervical cancer surviving five years after diagnosis

Denominator: Expected survival rate of a comparable group from the general population 
DELSA/HEA/WD/HWP(2007)4

Table 7. Cervical cancer five-year survival rate

\begin{tabular}{|c|c|c|c|c|c|c|c|}
\hline \multicolumn{6}{|c|}{ Cervical cancer } & \multirow{2}{*}{\multicolumn{2}{|c|}{$\begin{array}{l}\text { Mortality per } \\
100000 \text { women }\end{array}$}} \\
\hline \multirow[b]{3}{*}{ Country } & & $5-y$ & r survival rates & & & & \\
\hline & \multirow[b]{2}{*}{ Data year } & \multicolumn{2}{|c|}{ OSR (95\% Cl) } & \multicolumn{2}{|c|}{ RSR (95\% Cl) } & \multirow[b]{2}{*}{ Data year } & \multirow[b]{2}{*}{ Rate } \\
\hline & & Crude rates & $\begin{array}{c}\text { Age-standardised } \\
\text { rates }\end{array}$ & Crude rates & $\begin{array}{c}\begin{array}{c}\text { Age-standardised } \\
\text { rates }\end{array} \\
\end{array}$ & & \\
\hline Australia & $1992-1997$ & $72.8(71.4-74.1)$ & $75.7(74.3-77.0)$ & $74.6(73.2-75.9)$ & $77.6(76.9-79.0)$ & 2003 & 1.9 \\
\hline \multirow[t]{2}{*}{ Canada } & $1998-2003$ & $70.0(67.0-73.0)$ & $74.0(71.0-77.0)$ & $73.0(70.0-76.0)$ & $76.0(73.0-78.0)$ & 2002 & 1.8 \\
\hline & $1997-2002$ & $68.0(65.0-71.0)$ & $72.0(70.0-75.0)$ & $70.0(67.0-73.0)$ & $74.0(71.0-77.0)$ & & \\
\hline Czech Republic & 1994-1998 & $63.7(62.4-65.0)$ & & $68.4(67.0-69.8)$ & & 2004 & 5.4 \\
\hline Denmark & 2001-2005 & $70.0(66.0-75.0)$ & & $73.0(68.0-77.0)$ & & 2001 & 3.8 \\
\hline \multirow[t]{2}{*}{ Finland } & $1999-2003$ & $63.8(56.0-71.5)$ & & $70.7(62.0-78.7)$ & & 2004 & 1.4 \\
\hline & $1995-2001$ & $60.0(55.0-65.0)$ & & $65.7(60.8-70.7)$ & & & \\
\hline \multirow[t]{2}{*}{ France } & $1990-1994$ & & 62.7 (n.a-n.a) & & 65.9 (n.a-n.a) & 2003 & 1.8 \\
\hline & $1985-1989$ & & 59.0 (n.a-n.a) & & $64.0(61.8-70.1)$ & & \\
\hline Germany & $1993-1997$ & $62.0(57.0-67.0)$ & & $66.0(61.0-71.0)$ & & 2004 & 2.5 \\
\hline \multirow[t]{3}{*}{ Iceland } & $1996-2000$ & 74.1 (n.a-n.a) & & $76.4(64.6-88.2)$ & & 2004 & 1.4 \\
\hline & $1995-1999$ & 74.0 (n.a-n.a) & & $76.6(66.0-87.3)$ & & & \\
\hline & $1993-1997$ & 73.3 (n.a-n.a) & & $76.3(65.7-86.9)$ & & & \\
\hline \multirow[t]{3}{*}{ Ireland } & $1999-2004$ & $68.0(59.9-74.8)$ & & $70.4(62.0-77.4)$ & & 2005 & 3.4 \\
\hline & $1998-2002$ & & $55.0(50.9-59.1)$ & & $58.9(54.0-63.9)$ & & \\
\hline & 1994-1998 & $60.0(55.0-63.0)$ & & $62.0(58.0-66.0)$ & & & \\
\hline \multirow[t]{2}{*}{ Italy } & $1995-1999$ & $61.0(60.0-63.0)$ & & $66.0(64.0-68.0)$ & & 2002 & 0.8 \\
\hline & $1990-1994$ & & 59.0 (n.a-n.a) & & $64.0(64.5-68.7)$ & & \\
\hline Japan & 1993-1996 & & & $70.5(69.1-71.9)$ & & 2004 & 2.4 \\
\hline Korea & $1998-2002$ & $77.4(76.7-78.0)$ & & $80.1(79.4-80.7)$ & & 2004 & 4.5 \\
\hline \multirow{2}{*}{ Mexico } & $1997-2001$ & 40.7 (n.a-n.a) & & & & 1995 & 17.7 \\
\hline & $1997-1998$ & 30.1 (n.a-n.a) & & & & & \\
\hline \multirow[t]{2}{*}{ Netherlands $^{1}$} & $1996-2000$ & $66.8(62.6-70.8)$ & & $70.4(65.9-74.6)$ & & 2004 & 1.8 \\
\hline & 1993-1997 & $\begin{array}{l}<60 \text { yrs: } 75.0(67.0-83.0) \\
>60 \text { yrs: } 46.0(32.0-60.0)\end{array}$ & & $\begin{array}{l}<60 \text { yrs: } 76.0(68.0-84.0) \\
>60 \text { yrs: } 55.0(39.0-71.0)\end{array}$ & & & \\
\hline \multirow[t]{2}{*}{ New Zealand } & $1998-2003$ & $71.8(69.0-74.6)$ & $75.7(73.0-78.4)$ & $75.6(72.7-78.6)$ & $79.9(77.2-82.6)$ & 2001 & 2.8 \\
\hline & 1994-1999 & $66.8(63.5-70.1)$ & $69.3(62.9-75.7)$ & $70.5(67.1-74.0)$ & $72.9(70.1-75.8)$ & & \\
\hline \multirow[t]{3}{*}{ Norway } & $1998-2003$ & $68.3(66.1-70.5)$ & & $73.2(70.9-75.5)$ & & 2004 & 2.5 \\
\hline & $1996-2001$ & $62.3(56.8-67.8)$ & & $67.9(61.8-74.0)$ & & & \\
\hline & 1995-1999 & $67.9(65.5-70.3)$ & & $72.7(70.2-75.2)$ & & & \\
\hline Slovak Republic & 1998-2002 & 75.0 (n.a-n.a) & & & & 2002 & 5.9 \\
\hline \multirow[t]{2}{*}{ Sweden } & $1999-2004$ & $65.2(62.8-67.5)$ & & $70.7(68.1-73.3)$ & & 2002 & 2.4 \\
\hline & $1996-2001$ & $66.0(61.6-70.5)$ & & $69.2(64.5-73.9)$ & & & \\
\hline Switzerland & 1990-1994 & 66.0 (n.a-n.a) & & 72.0 (n.a-n.a) & & 2004 & 1.6 \\
\hline \multirow[t]{2}{*}{ United Kingdom } & $1998-2001$ & 70.0 (n.a-n.a) & & 72.0 (n.a-n.a) & & 2004 & 2.5 \\
\hline & 1995-1999 & 68.0 (n.a-n.a) & & 67.0 (n.a-n.a) & $62.9(60.4-65.4)$ & & \\
\hline \multirow[t]{2}{*}{ United States } & $1998-2002$ & $67.8(65.6-69.9)$ & & $72.0(69.8-74.1)$ & & 2002 & 2.2 \\
\hline & $1994-1998$ & $73.6(70.1-77.1)$ & & $75.4(69.4-81.5)$ & & & \\
\hline
\end{tabular}


DELSA/HEA/WD/HWP(2007)4

\begin{tabular}{|c|c|c|c|c|c|c|c|}
\hline \multicolumn{6}{|c|}{ Non-OECD EU countries } & \multicolumn{2}{|c|}{$\begin{array}{c}\text { Mortality per } 100000 \\
\text { women }\end{array}$} \\
\hline \multirow[b]{2}{*}{ Country } & \multirow[b]{2}{*}{ Data year } & \multicolumn{2}{|c|}{ OSR (95\% Cl) } & \multicolumn{2}{|c|}{$\operatorname{RSR}(95 \% \mathrm{Cl})$} & \multirow[b]{2}{*}{ Data year } & \multirow[b]{2}{*}{ Rate } \\
\hline & & Crude rates & $\begin{array}{l}\text { Age-standardised } \\
\text { rates }\end{array}$ & Crude rates & $\begin{array}{l}\text { Age-standardised } \\
\text { rates }\end{array}$ & & \\
\hline Latvia & 1999-2003 & 50.0 (n.a-n.a) & & 58.9 (n.a-n.a) & & 2005 & 8.4 \\
\hline Malta & 1993-1994 & & & & $64.0(52.0-81.0)$ & 2005 & 1.0 \\
\hline
\end{tabular}

Notes:

$\mathrm{Cl}$ stands for "Confidence Interval". Observed survival rates are an estimation of the probability of a patient still alive five years after being diagnosed of cancer based on the actual data available. Relative survival rates adjusts this probability of surviving a cancer diagnosis by the general probability of surviving attributable to any member of the same age group independently of whether they do suffer cancer Thus the $95 \%$ confidence interval $(\mathrm{Cl})$ illustrates the degree of variability associated with these estimates. Wide confidence intervals indicate high variability, therefore, these estimates should be interpreted with due caution. When estimates are based on a small number of cases, it is more likely that observed differences are due to random, rather than systematic influences.

Reference population for age-standardised rates: OECD 1980, except for France, Ireland, Italy and Malta who refer to the Eurocare-3 population and the Slovak Republic to the Standard European population.

"n.a" stands for "no data available".

1. Data is not national, but has been weighted by the incidence they represent upon the nation. See Sources and Methods for more information.

Source: HCQI Project, 2007. Mortality data: OECD Health Data 2007, July 07 
DELSA/HEA/WD/HWP(2007)4

Cervical cancer five-year survival rate, sources and methods

\begin{tabular}{|c|c|c|c|c|c|c|c|}
\hline Country & Source & Diagnosis Code & $\begin{array}{c}\text { Age } \\
\text { groups } \\
\text { included }\end{array}$ & $\begin{array}{l}\text { Additional } \\
\text { years }\end{array}$ & Is this a national registry? & $\begin{array}{l}\text { Reference } \\
\text { population }\end{array}$ & Comments \\
\hline Canada & Canadian Cancer Registry & $\begin{array}{l}\text { Canadian data coded } \\
\text { using ICDO-3 (not } \\
\text { ICD 9/10) }\end{array}$ & $15-99$ & & Yes & $\begin{array}{l}1980 \text { OECD } \\
\text { population }\end{array}$ & $\begin{array}{l}\text { Missing cases treated as: we rely on record } \\
\text { linkage for vital status (i.e. passive follow- } \\
\text { up) and as such we cannot identify cases } \\
\text { lost to follow-up. So we don't "handle" them } \\
\text { in any way. Practically speaking there will } \\
\text { be people who die outside of the country } \\
\text { and whose death is not recorded in the } \\
\text { national mortality database. }\end{array}$ \\
\hline $\begin{array}{l}\text { Czech } \\
\text { Republic }\end{array}$ & $\begin{array}{l}\text { National Cancer Registry } \\
\text { of the Czech Republic; } \\
\text { Vital Statistics (Czech } \\
\text { Statistical Office) }\end{array}$ & ICD-10 C53 & & $1980-1998$ & Yes & & Missing cases treated as: survivors \\
\hline Denmark & National Cancer Registry & ICD-10 C53 & & 1977 & Yes & & $\begin{array}{l}\text { Missing cases treated as: dropped out. The } \\
\text { calculations are based on unique } \\
\text { identifiers, why few people are lost to } \\
\text { follow-up. }\end{array}$ \\
\hline Finland & Finnish Cancer Register & ICD-10 C53 & & 1953-2004 & Yes & & $\begin{array}{l}\text { Very few cases are behind each age group } \\
\text { in "Age specific rate" - } 0-6 \text { cases except for } \\
25-39 \text { years old ( } 29 \text { cases) Figures are } \\
\text { therefore very coincidental. Missing cases } \\
\text { treated as: no losses to follow-up }\end{array}$ \\
\hline France & $\begin{array}{l}\text { EUROCARE-3: Electronic } \\
\text { availability of EUROCARE } \\
3 \text { data: a tool for further } \\
\text { analysis P Roazzi, Annals } \\
\text { of Oncology } 14150-155, \\
\text { 2003. National death } \\
\text { causes database/Available } \\
\text { Eurostat Database. }\end{array}$ & ICD-10 C53 & $15+$ & & $\begin{array}{l}3 \text { regional registries : } \\
\text { Calvados, Côte d'Or, Bas- } \\
\text { Rhin, representing 2,162.000 } \\
\text { persons, i.e. } 3,8 \% \text { of French } \\
\text { population in } 1990\end{array}$ & ICSS population & $\begin{array}{l}\text { French network of cancer registries survival } \\
\text { data fro } 1988 \text { to } 1997 \text { follow-up to } 2002 \text { will } \\
\text { be published soon }\end{array}$ \\
\hline Germany & Saarland Cancer Registry & ICD-9 180 & $15-89$ & & $\begin{array}{l}\text { State of Saarland. Not } \\
\text { generalisable }\end{array}$ & & \\
\hline
\end{tabular}


DELSA/HEA/WD/HWP(2007)4

\begin{tabular}{|c|c|c|c|c|c|c|c|}
\hline Country & Source & Diagnosis Code & $\begin{array}{c}\text { Age } \\
\text { groups } \\
\text { included }\end{array}$ & $\begin{array}{l}\text { Additional } \\
\text { years }\end{array}$ & Is this a national registry? & $\begin{array}{l}\text { Reference } \\
\text { population }\end{array}$ & Comments \\
\hline Iceland & Icelandic Cancer Registry & ICD-10 C53 & & $1955-1995$ & Yes & & Missing cases treated as: non-survivors \\
\hline Ireland & $\begin{array}{l}\text { NCRI. Irish National } \\
\text { Cancer Registry }\end{array}$ & ICD-10 C53 & $15-99$ & & & $\begin{array}{l}\text { Eurocare } 3 \\
\text { standard patient } \\
\text { populations }\end{array}$ & $\begin{array}{l}\text { Missing cases treated as: follow up is } \\
\text { passive, by means of matching registered } \\
\text { cases to death certificates. Currently } \\
\text { matching completed to } 31 \text { December } 2003 \\
\text { (censoring date) and patients not matched } \\
\text { by this date (known to have died by this } \\
\text { date) are assumed to be alive }\end{array}$ \\
\hline Italy & Registro Nazionale Tumori & ICD-10 C53 & $14+$ & & Yes & & Missing cases treated as: dropped out \\
\hline Japan & $\begin{array}{l}\text { Tsukuma H, Ajiki W, loka } \\
\text { A, Oshima A, and } \\
\text { Research Group of } \\
\text { Population-Based Cancer } \\
\text { Registry of Japan, Survival } \\
\text { of cancer patients } \\
\text { diagnosed in 1993-96: } \\
\text { collaborative study of } \\
\text { population-based cancer } \\
\text { registries in Japan, } \\
\text { Japanese Journal of } \\
\text { Clinical Oncology, 36: 602- } \\
\text { 607, } 2006\end{array}$ & ICD-9 180 & & & & & $\begin{array}{l}\text { Includes both cervical and endometrial } \\
\text { cancer }\end{array}$ \\
\hline
\end{tabular}


DELSA/HEA/WD/HWP(2007)4

\begin{tabular}{|c|c|c|c|c|c|c|c|}
\hline Country & Source & Diagnosis Code & $\begin{array}{c}\text { Age } \\
\text { groups } \\
\text { included }\end{array}$ & $\begin{array}{c}\text { Additional } \\
\text { years }\end{array}$ & Is this a national registry? & $\begin{array}{l}\text { Reference } \\
\text { population }\end{array}$ & Comments \\
\hline Korea & $\begin{array}{l}\text { Korea Central Cancer } \\
\text { Registry Source of } \\
\text { mortality data: Annual } \\
\text { Report on the Cause of } \\
\text { Death Statistics. Korea } \\
\text { National Statistical Office, } \\
2006 \text {. }\end{array}$ & ICD-10 C53 & & & Yes & & $\begin{array}{l}\text { 1. The Korea Central Cancer Registry has } \\
\text { been collaborating with the Gynaecologic } \\
\text { Oncology Committee of Korean Society of } \\
\text { Obstetrics and Gynaecology to produce } \\
\text { national cervical cancer statistics. } 2 \text {. Since } \\
\text { our cancer incidence DB is under review of } \\
\text { the International Agency for Research on } \\
\text { Cancer (IARC), statistical figures may } \\
\text { change after the completion of the review. } \\
\text { Missing cases treated as: dropped out }\end{array}$ \\
\hline Mexico & $\begin{array}{l}\text { SUAVE: Sistema Unico } \\
\text { Automatizado de Vigilancia } \\
\text { Epidemiologica IMSS. } \\
\text { Source of mortality data: } \\
\text { Sistema Nacional de } \\
\text { Mortalidad IMSS } \\
\text { (SISMOR). }\end{array}$ & ICD-10 C53 & & & Yes & & $\begin{array}{l}\text { We can not obtain rates by age group, } \\
\text { because data are total cases not disclosed, } \\
\text { we will obtain this information near the } \\
2006 \text { close. Missing cases treated as: non- } \\
\text { survivors }\end{array}$ \\
\hline Netherlands & $\begin{array}{l}\text { National Institute of Public } \\
\text { Health and the } \\
\text { Environment. Source of } \\
\text { Mortality data: Statline. } \\
\text { Voorbrug: Statistics } \\
\text { Netherlands, } 2006 \text { Website } \\
\text { http://statline.cbs.nl/StatW } \\
\text { eb/Start.asp?|p=Search/Se } \\
\text { arch\&LA=EN\&DM=SLEN. }\end{array}$ & ICD-10 C53 & All & & $\begin{array}{l}\text { No. In the Netherlands, the } \\
\text { National Cancer Registry of } \\
\text { the Association of } \\
\text { Comprehensive Cancer } \\
\text { Centres (CCC) delivers } \\
\text { incidence rates. However, } \\
\text { survival rates can not be } \\
\text { calculated for the whole } \\
\text { country. At this moment, three } \\
\text { Comprehensive Cancer } \\
\text { Centres have data on cancer } \\
\text { survival. In a few years, all } \\
\text { CCC's will deliver data on } \\
\text { cancer survival. Data } \\
\text { therefore cover over } 40 \% \text { of } \\
\text { Dutch population. Data have } \\
\text { been weighted by the } \\
\text { incidence of the CCC into the } \\
\text { total incidence of the country. }\end{array}$ & & $\begin{array}{l}\text { Missing cases treated as: the CCC's obtain } \\
\text { data of persons in the population who died. } \\
\text { These data are obtained from the Municipal } \\
\text { Basis Registry, which is to a large extent } \\
\text { complete. Patients, of whom no death } \\
\text { report is obtained, are still alive. Emigrants } \\
\text { are censored. Overall, almost } 100 \% \text { of } \\
\text { follow-up is complete. }\end{array}$ \\
\hline
\end{tabular}


DELSA/HEA/WD/HWP(2007)4

\begin{tabular}{|l|l|l|l|l|l|l|l|}
\hline \multicolumn{1}{|c|}{ Country } & \multicolumn{1}{|c|}{ Source } & Diagnosis Code & $\begin{array}{c}\text { Age } \\
\text { groups } \\
\text { included }\end{array}$ & $\begin{array}{l}\text { Additional } \\
\text { years }\end{array}$ & Is this a national registry? & $\begin{array}{c}\text { Reference } \\
\text { population }\end{array}$ & Comments \\
\hline $\begin{array}{l}\text { New } \\
\text { Zealand }\end{array}$ & $\begin{array}{l}\text { New Zealand Cancer } \\
\text { Registry (NZCR). Source } \\
\text { of mortality data: NZHIS } \\
\text { Mortality data collection } \\
\text { 1998-2006 }\end{array}$ & $\begin{array}{l}\text { ICD-10-AM } \\
\text { (Australian } \\
\text { Modification) C53 }\end{array}$ & & $1994-2006$ & Yes & $\begin{array}{l}\text { OECD standard } \\
\text { population 1980 } \\
\text { (only for relative } \\
\text { rates) }\end{array}$ & $\begin{array}{l}\text { Missing cases treated as: passive follow- } \\
\text { up, losses. DCO (Death certificate only) } \\
\text { cases were excluded }\end{array}$ \\
\hline Norway & Cancer Registry of Norway & ICD-10 C53 & & & Yes & Missing cases treated as: Dropped out \\
\hline $\begin{array}{l}\text { Slovak } \\
\text { Republic }\end{array}$ & National cancer registry & ICD-10 C53 & & $\begin{array}{l}\text { Since } \\
1970\end{array}$ & Yes & $\begin{array}{l}\text { standard } \\
\text { European } \\
\text { population }\end{array}$ & $\begin{array}{l}\text { Diagnosed in 1998; survival 31.12.2002 } \\
\text { Missing cases treated as: returned to the } \\
\text { do not have missing cases. }\end{array}$ \\
\hline Sweden & $\begin{array}{l}\text { The Swedish Cancer } \\
\text { Register }\end{array}$ & ICD-9 180 & & $1961-2002$ & yes & $\begin{array}{l}\text { Missing cases treated as: censored in a } \\
\text { survival analyses (actuarial method) }\end{array}$ \\
\hline $\begin{array}{l}\text { United } \\
\text { Kingdom }\end{array}$ & $\begin{array}{l}\text { Office for National } \\
\text { Statistics/Department of } \\
\text { Health }\end{array}$ & ICD-9 180 & $15-99$ & & Data are for England & OECD 1980 & \\
\hline $\begin{array}{l}\text { United } \\
\text { States }\end{array}$ & $\begin{array}{l}\text { Surveillance Epidemiology } \\
\text { and End Results (SEER) } \\
\text { Program } \\
\text { (www.ser.cancer.gov) } \\
\text { SEER*Stat }\end{array}$ & & & & Generalisable to nation & OECD 1980 & \\
\hline
\end{tabular}

\begin{tabular}{|l|l|l|c|c|c|c|c|}
\hline \multicolumn{9}{|c|}{ Source } & Diagnosis Code & $\begin{array}{c}\text { Age } \\
\text { groups } \\
\text { included }\end{array}$ & $\begin{array}{l}\text { Additional } \\
\text { years }\end{array}$ & Is this a national registry? & $\begin{array}{c}\text { Reference } \\
\text { population }\end{array}$ & \multicolumn{1}{c|}{ Comments } \\
\hline Latvia & $\begin{array}{l}\text { Cancer Registry. Source of } \\
\text { mortality data: National } \\
\text { Death Causes Database. }\end{array}$ & ICD-10 C53 & & & Yes & $\begin{array}{l}\text { Missing cases treated as: In the calculation } \\
\text { from "missing cases" 1/2 treated as } \\
\text { survivors and other 1/2 as non-survivors. }\end{array}$ \\
\hline
\end{tabular}


DELSA/HEA/WD/HWP(2007)4

\begin{tabular}{|c|c|c|c|c|c|c|c|}
\hline \multicolumn{8}{|c|}{ Non-OECD EU countries } \\
\hline Country & Source & Diagnosis Code & $\begin{array}{c}\text { Age } \\
\text { groups } \\
\text { included }\end{array}$ & $\begin{array}{l}\text { Additional } \\
\text { years }\end{array}$ & Is this a national registry? & $\begin{array}{l}\text { Reference } \\
\text { population }\end{array}$ & Comments \\
\hline Malta & $\begin{array}{l}\text { Eurocare-3: Survival of } \\
\text { Cancer Patients in } \\
\text { Eurocare: the EUROCARE- } \\
3 \text { Study, Annals of } \\
\text { Oncology, Vol. 14, 2003, } \\
\text { Supplement 5. Source of } \\
\text { mortality data: WHO-HFA: } \\
\text { http://data.euro.who.int/hfad } \\
\text { b/ }\end{array}$ & & & & & & $\begin{array}{l}\text { Mortality figures are age-standardised } \\
\text { using European Standard Population }\end{array}$ \\
\hline
\end{tabular}




\section{Cervical cancer screening rate}

\section{Operational Definition}

Numerator: Number of women ages 20-69 reporting cervical cancer screening within the past 3 years or number of women age 20-69 screened for cervical cancer through an organised programme.

Denominator: Number of women ages 20-69 answering survey question or participating in an organised screening programme.

Table 8. Cervical cancer screening rate

\begin{tabular}{|c|c|c|c|c|c|c|c|c|c|}
\hline \multirow{2}{*}{ Country } & \multicolumn{7}{|c|}{$\%$ women $20-69$ screened } & \multirow{2}{*}{$\begin{array}{l}\text { Recall period } \\
\text { (years) }\end{array}$} & \multirow{2}{*}{$\begin{array}{l}\text { Survey or } \\
\text { screening }\end{array}$} \\
\hline & 2000 & 2001 & 2002 & 2003 & 2004 & 2005 & 2006 & & \\
\hline Australia & 61.1 & 61 & 60.6 & 60.5 & & & & 2 & screening program \\
\hline Belgium ${ }^{1}$ & 59.5 & 63.2 & 61 & 61 & 63.1 & & & 3 & screening program \\
\hline Finland $^{4}$ & & 67.5 & 71.8 & 71.5 & & & & 5 & screening program \\
\hline Germany $^{5}$ & & & 55.9 & & & & & 5 & screening program \\
\hline Hungary $^{6}$ & 28.4 & 27.3 & 27.3 & 27.5 & 28.1 & & & 3 & screening program \\
\hline Iceland & 74 & 74 & 74 & 74 & 73 & 72 & & 3 & screening program \\
\hline Ireland $^{7}$ & & & & 70.1 & 65.5 & 60.9 & & 5 & screening program \\
\hline Mexico $^{9}$ & & & 38.9 & & & 37.1 & & & screening program \\
\hline Netherlands ${ }^{10}$ & 66.9 & 67.1 & 66.4 & 68.8 & 68.9 & 69.6 & & 5 & screening program \\
\hline New Zealand & 73.1 & 72.7 & 72.2 & 72 & & & & 3 & screening program \\
\hline Norway ${ }^{11}$ & 70.3 & & & & 72.5 & & & 3 & screening program \\
\hline Sweden $^{12}$ & & & 72 & & & & & 5 & screening program \\
\hline United Kingdom $^{13}$ & 67.2 & 66.1 & 71.2 & 70.8 & 70.3 & 69.7 & 69.8 & 3.5 & screening program \\
\hline Canada $^{2}$ & 72.7 & & & 74.1 & & 72.8 & & 3 & survey \\
\hline Czech Republic ${ }^{3}$ & & & 38.8 & & & & & 1 & survey \\
\hline Denmark & 69.7 & & & & & 69.4 & & 3 & survey \\
\hline France & 54 & & & 74.9 & & & & 2 & survey \\
\hline Italy $^{8}$ & 45.1 & & & & & 36.7 & & 3 & survey \\
\hline Japan & & 22.6 & & & 23.7 & & & 1 & survey \\
\hline Korea & & & & & & 40.6 & & 2 & survey \\
\hline Poland & & & & & 49 & & & & survey \\
\hline United States & 84.8 & & & 82.6 & & & & 3 & survey \\
\hline
\end{tabular}

Notes:

1. 25-64 years.

2. 18-69 years.

3. $15+$ years.

4. 30-60 years.

5. $20-45$ years.

6. 25-65 years.

7. 20-60 years.

8. 25-69 years

9. 25-64 years for 2002; 20-69 years for 2005 .

10. 18-64 for 2001-2004 data, 30-60 other years.

11. $25-67$ years.

12. 23-60 years.

13. $25-64$ years

Source: OECD Health Data 2007, July 07

\begin{tabular}{|l|c|c|c|c|}
\hline \multicolumn{5}{|c|}{ Non-OECD EU countries } \\
\hline Country & Year & $\%$ & $\begin{array}{c}\text { Recall period } \\
\text { (years) }\end{array}$ & $\begin{array}{c}\text { Screening } \\
\text { Program }\end{array}$ \\
\hline Malta & 67.3 & 2002 & 3 & survey \\
\hline
\end{tabular}

Source: Health Interview Survey: http://www.sahha.gov.mt/pages.aspx?page=383 
Cervical cancer screening rate, sources and method

http://www.ecosante.org/OCDEENG/370020.html

\section{Colorectal cancer five-year survival rate}

Operational Definition

A. 5-year observed survival rate (OSR), colorectal cancer (Diagnostic code: ICD-9 C:153.xx, 154.xx; ICD10: C18.xx, C19.xx, C20.xx)

Numerator: Number of people diagnosed with colorectal cancer surviving five years after diagnosis.

Denominator: Number of people diagnosed with colorectal cancer.

B. 5-year relative survival rate (RSR), colorectal cancer (Diagnostic code: ICD-9 C:153.xx, 154.xx; ICD10: C18.xx, C19.xx, C20.xx)

Numerator: Observed rate of people diagnosed with colorectal cancer surviving five years after diagnosis.

Denominator: Expected survival rate of a comparable group from the general population. 
Table 9. Colorectal cancer five-year survival rate

\begin{tabular}{|c|c|c|c|c|c|c|c|}
\hline \multicolumn{6}{|c|}{ Colorectal cancer } & \multirow{2}{*}{\multicolumn{2}{|c|}{$\begin{array}{l}\text { Mortality per } \\
100000 \text { women }\end{array}$}} \\
\hline \multirow[b]{3}{*}{ Country } & & \multirow{2}{*}{\multicolumn{2}{|c|}{$\begin{array}{l}\text { 5-year survival rates } \\
\text { OSR }(95 \% \mathrm{Cl}) \\
\end{array}$}} & & & & \\
\hline & \multirow[b]{2}{*}{ Data year } & & & \multicolumn{2}{|c|}{$\operatorname{RSR}(95 \% \mathrm{CI})$} & \multirow{2}{*}{ Data year } & \multirow{2}{*}{ Rate } \\
\hline & & Crude rates & Age-standardised rates & Crude rates & Age-standardised rates & & \\
\hline Australia & $1992-1997$ & $56.0(53.9-57.8)$ & $59.0(57.1-64.0)$ & $58.5(59.2-57.8)$ & $62.0(59.9-64.0)$ & 2003 & 18.2 \\
\hline \multirow{2}{*}{ Canada } & $1998-2003$ & $50.0(49.0-51.0)$ & $60.0(57.0-63.0)$ & $60.0(59.0-60.0)$ & $63.0(60.0-65.0)$ & 2002 & 18.4 \\
\hline & 1997-2002 & $50.0(49.0-51.0)$ & $59.0(56.0-62.0)$ & $60.0(59.0-61.0)$ & $62.0(59.0-65.0)$ & & \\
\hline Czech Republic & 1994-1998 & $32.1(31.4-32.9)$ & & $41.2(40.3-42.2)$ & & 2004 & 33.9 \\
\hline Denmark & 2001-2005 & $\begin{array}{l}\text { colon: } 41.0(39.0-44.0) \\
\text { rectum: } 44.0(40.0-47.0) \\
\end{array}$ & & $\begin{array}{l}\text { colon: } 51.0(48.0-54.0) \\
\text { rectum: } 54.0(50.0-57.0) \\
\end{array}$ & & 2001 & 27.3 \\
\hline \multirow{2}{*}{ Finland } & 1999-2003 & $47.0(46.0-49.0)$ & & $59.2(57.7-60.7)$ & & 2004 & 12.6 \\
\hline & $1995-2000$ & $43.1(40.7-45.5)$ & & $56.3(53.3-59.4)$ & & & \\
\hline \multirow[t]{2}{*}{ France } & $1990-1994$ & & $\begin{array}{l}\text { men: } 45.5 \text { (n.a-n.a) } \\
\text { women: } 54.1 \text { (n.a-n.a) }\end{array}$ & & $\begin{array}{c}\text { men: } 55.8(53.4-58.2) \\
\text { women: } 61.7(59.3-64.1)\end{array}$ & 2003 & 17.8 \\
\hline & 1985-1989 & & 41. 0 (n.a-n.a) & & 53.0 (n.a-n.a) & & \\
\hline Germany & 1993-1997 & $\begin{array}{c}\text { men: } 43.0(41.0-45.0) \\
\text { women: } 45.0(42.0-47.0)\end{array}$ & & $\begin{array}{c}\text { men: } 55.0(52.0-58.0) \\
\text { women: } 56.0(53.0-59.0)\end{array}$ & & 2004 & 20.5 \\
\hline \multirow{3}{*}{ Iceland } & $1996-2000$ & $\begin{array}{c}\text { men: } 47.8 \text { (n.a-n.a) } \\
\text { women: } 43.6 \text { (n.a-n.a) }\end{array}$ & & $\begin{array}{c}\text { men: } 60.2(51.5-68.9) \\
\text { women: } 52.9(43.5-62.3)\end{array}$ & & 2004 & 13.8 \\
\hline & 1995-1999 & $\begin{array}{c}\text { men: } 46.5 \text { (n.a-n.a) } \\
\text { women: } 42.7 \text { (n.a-na.) }\end{array}$ & & $\begin{array}{l}\text { men: } 58.9(51.0-66.8) \\
\text { women: } 52.5(44.0-61.0)\end{array}$ & & & \\
\hline & 1993-1997 & $\begin{array}{c}\text { men: } 40.1 \text { (n.a-n.a) } \\
\text { women: } 43.6 \text { (n.a-n.a) }\end{array}$ & & $\begin{array}{c}\text { men: } 51.1(42.7-59.6) \\
\text { women: } 53.5(44.7-62.4) \\
\end{array}$ & & & \\
\hline \multirow{3}{*}{ Ireland } & 1999-2004 & $\begin{array}{c}\text { men: } 41.5(38.4-44.6) \\
\text { women: } 39.5(36.0-43.1)\end{array}$ & & $\begin{array}{c}\text { men: } 52.2(48.3-56.1) \\
\text { women: } 47.8(43.5-52.1)\end{array}$ & & 2005 & 21.0 \\
\hline & 1998-2002 & & $\begin{array}{c}\text { men: } 34.1(32.3-35.8) \\
\text { women: } 41.1(39.1-43.0)\end{array}$ & & $\begin{array}{c}\text { men: } 47.3(44.5-50.0) \\
\text { women: } 51.2(48.6-44.5)\end{array}$ & & \\
\hline & 1994-1998 & 41.0 (n.a-n.a) & & $62.0(58.0-66.0)$ & & & \\
\hline \multirow{2}{*}{ Italy } & $1995-1999$ & $46.0(46.0-47.0)$ & & $57.0(57.0-58.0)$ & & 2002 & 17.7 \\
\hline & 1990-1994 & & 42.0 (n.a-n.a) & & 52.0 (n.a-n.a) & & \\
\hline Japan & 1993-1996 & & & $\begin{array}{c}\text { all: } 67.5(67.2-67.8) \\
\text { men: } 69.5(69.1-69.9) \\
\text { women: } 64.6(64.2-65.0) \\
\end{array}$ & & 2004 & 18.0 \\
\hline Korea & $1998-2002$ & $53.7(53.2-54.2)$ & & $60.2(59.6-60.7)$ & & 2004 & 15.2 \\
\hline Mexico & $1997-1998$ & 47.5 (n.a-n.a) & & & & 1995 & 4.9 \\
\hline \multirow[t]{2}{*}{ Netherlands ${ }^{1}$} & $1996-2000$ & $\begin{array}{c}\text { all: } 45.6(42.5-48.6) \\
\text { men: } 44.1(41.2-47.1) \\
\text { women: } 47.1(43.9-50.1)\end{array}$ & & $\begin{array}{c}\text { all: } 56.7(52.8-60.6) \\
\text { men: } 56.4(52.4-60.2) \\
\text { women: } 57.1(53.2-60.9)\end{array}$ & & 2004 & 20.3 \\
\hline & 1993-1997 & $\begin{array}{l}\text { colon: } 48.0(46.0-50.0) \\
\text { rectal: } 46.0(42.0-50.0)\end{array}$ & & $\begin{array}{l}\text { colon: } 60.0(56.0-64.0) \\
\text { rectal: } 56.0(52.0-60.0) \\
\end{array}$ & & & \\
\hline \multirow[t]{2}{*}{ New Zealand } & 1998-2003 & $\begin{array}{c}\text { all: } 49.2(48.4-50.1) \\
\text { men: } 47.5(46.3-48.8) \\
\text { women: } 51.0(49.8-52.3)\end{array}$ & $58.5(57.4-59.6)$ & $\begin{array}{c}\text { all: } 60.4(59.3-61.5) \\
\text { men: } 59.0(57.4-60.5) \\
\text { women: } 61.8(60.3-63.4)\end{array}$ & $\begin{array}{c}\text { all: } 61.2(60.1-62.3) \\
\text { men: } 63.3(61.7-64.9) \\
\text { women: } 59.7(58.1-61.3)\end{array}$ & 2001 & 27.2 \\
\hline & 1994-1999 & $47.6(46.5-48.6)$ & $52.8(51.1-54.6)$ & $58.9(57.6-60.2)$ & $60.8(58.0-63.6)$ & & \\
\hline \multirow{3}{*}{ Norway } & $1998-2003$ & $43.1(42.3-43.9)$ & & $56.6(55.6-57.7)$ & & 2004 & 23.1 \\
\hline & $1996-2001$ & $44.0(42.1-45.8)$ & & $59.8(57.3-62.3)$ & & & \\
\hline & 1995-1999 & $43.8(43.0-44.6)$ & & $57.3(56.2-58.3)$ & & & \\
\hline Slovak Republic & 1998-2002 & $\begin{array}{l}\text { men: } 51.0 \text { (n.a-n.a) } \\
\text { women: } 54.0 \text { (n.a-na.) }\end{array}$ & & & & 2002 & 31.0 \\
\hline \multirow{2}{*}{ Sweden } & 1999-2004 & $46.4(45.6-47.2)$ & & $58.4(57.4-59.4)$ & & 2002 & 17.2 \\
\hline & $1996-2001$ & $45.9(44.4-47.4)$ & & $58.3(56.5-60.2)$ & & & \\
\hline Switzerland & 1990-1994 & $\begin{array}{l}\text { men: } 48.0 \text { (n.a-n.a) } \\
\text { women: } 51.0 \text { (n.a-n.a) }\end{array}$ & & $\begin{array}{l}\text { men: } 59.0 \text { (n.a-n.a) } \\
\text { women: } 62.0 \text { (n.a-n.a) }\end{array}$ & & 2004 & 14.2 \\
\hline \multirow{2}{*}{ United Kingdom $^{2}$} & $1998-2001$ & $55.0(54.9-55.1)$ & & $57.0(56.9-57.1)$ & & 2004 & 17.9 \\
\hline & 1995-1999 & $53.0(52.9-53.0)$ & & 49.0 (n.a-n.a) & $48.5(47.3-49.6)$ & & \\
\hline \multirow[t]{2}{*}{ United States } & $1998-2002$ & $\begin{array}{c}\text { all: } 50.9(50.1-51.6) \\
\text { men: } 51.0(49.8-52.1) \\
\text { women: } 50.7(49.7-51.7)\end{array}$ & & $\begin{array}{c}\text { all: } 64.4(63.4-65.3) \\
\text { men: } 65.2(63.8-66.6) \\
\text { women: } 63.7(62.3-65.1)\end{array}$ & & 2002 & 16.3 \\
\hline & 1994-1998 & 54.0 (n.a-n.a) & & 58.0 (n.a-n.a) & & & \\
\hline
\end{tabular}


DELSA/HEA/WD/HWP(2007)4

\begin{tabular}{|c|c|c|c|c|c|c|c|}
\hline \multicolumn{6}{|c|}{ Non-OECD EU countries } & \multicolumn{2}{|c|}{$\begin{array}{l}\text { Mortality per } 100000 \\
\text { women }\end{array}$} \\
\hline \multirow{2}{*}{ Country } & \multirow{2}{*}{ Data year } & \multicolumn{2}{|c|}{ OSR $(95 \% \mathrm{Cl})$} & \multicolumn{2}{|c|}{ RSR $(95 \% \mathrm{Cl})$} & \multirow{2}{*}{ Data year } & \multirow[b]{2}{*}{ Rate } \\
\hline & & Crude rates & Age-standardised rates & Crude rates & Age-standardised rates & & \\
\hline Latvia & $1999-2003$ & 28.9 (n.a-n.a) & & 36.2 (n.a-n.a) & & 2005 & 30.1 \\
\hline \multirow[t]{2}{*}{ Malta } & 1998-2002 & & & $\begin{array}{c}\text { men: } 54.0(45.0-62.0) \\
\text { women: } 49.0(41.0-57.0)\end{array}$ & & 2005 & $\begin{array}{c}\text { men: } 23.8 \\
\text { women: } \\
15.6\end{array}$ \\
\hline & 1993-1994 & & & & $\begin{array}{c}\text { men: } 39.0(28.0-53.0) \\
\text { women: } 54.0(43.0-68.0)\end{array}$ & & \\
\hline
\end{tabular}

Notes:

$\mathrm{Cl}$ stands for "Confidence Interval". Observed survival rates are an estimation of the probability of a patient to be alive five years after being diagnosed of cancer based on the actual data available. Relative survival rates adjusts this probability of surviving a cancer diagnosis by the general probability of surviving attributable to any member of the same age group independently of whether they do suffer cancer Thus the $95 \%$ confidence interval $(\mathrm{Cl})$ illustrates the degree of variability associated with these estimates. Wide confidence intervals indicate high variability, therefore, these estimates should be interpreted with due caution. When estimates are based on a small number of cases, it is more likely that observed differences are due to random, rather than systematic influences. "n.a" stands for "no data available".

Reference population for age-standardised rates: OECD 1980, except for France, Ireland, Italy and Malta who refer to the Eurocare-3 population and the Slovak Republic to the Standard European population.

1. Data are not national, but have been weighted by the incidence they represent. See Sources and Methods for more information.

2. Data refer to colon cancer.

Source: HCQI Indicators, 2007. Mortality data: OECD Health Data 2007, July 07 
DELSA/HEA/WD/HWP(2007)4

Colorectal cancer five-year survival rate, sources and methods

\begin{tabular}{|c|c|c|c|c|c|c|c|}
\hline Country & Source & $\begin{array}{l}\text { Diagnosis } \\
\text { Code }\end{array}$ & $\begin{array}{l}\text { Age groups } \\
\text { included }\end{array}$ & $\begin{array}{l}\text { Additional } \\
\text { years }\end{array}$ & $\begin{array}{l}\text { Is this a national } \\
\text { registry? }\end{array}$ & $\begin{array}{l}\text { Reference } \\
\text { population }\end{array}$ & Comments \\
\hline Canada & Canadian Cancer Registry & $\begin{array}{l}\text { Canadian } \\
\text { data coded } \\
\text { using ICDO-3 } \\
\text { (not ICD } \\
9 / 10)\end{array}$ & $15-99$ & & Yes & $\begin{array}{l}1980 \text { OECD } \\
\text { population }\end{array}$ & $\begin{array}{l}\text { Missing cases treated as: we rely on record } \\
\text { linkage for vital status (i.e. passive follow-up) } \\
\text { and as such we cannot identify cases lost to } \\
\text { follow-up. So we don't "handle" them in any } \\
\text { way. Practically speaking there will be people } \\
\text { who die outside of the country and whose } \\
\text { death is not recorded in the national mortality } \\
\text { database. }\end{array}$ \\
\hline $\begin{array}{l}\text { Czech } \\
\text { Republic }\end{array}$ & $\begin{array}{l}\text { National Cancer Registry of the Czech } \\
\text { Republic; Vital Statistics (Czech Statistical } \\
\text { Office) }\end{array}$ & $\begin{array}{l}\text { ICD-10 C18, } \\
\text { C19, C20. }\end{array}$ & & $1980-1998$ & Yes & & $\begin{array}{l}\text { Data relates to men only, values for women } \\
\text { are also available. Also available separately } \\
\text { data for colon and rectum cancer. }\end{array}$ \\
\hline & & & & & & & Missing cases treated as: survivors \\
\hline Denmark & National Cancer Registry & $\begin{array}{l}\text { ICD-10 C18, } \\
\text { C19, C20 }\end{array}$ & & $1981-1995$ & & & Missing cases are dropped out. \\
\hline Finland & Finnish Cancer Register & $\begin{array}{l}\text { ICD-10 C18, } \\
\text { C19, C20. }\end{array}$ & & 1953-2004 & Yes & & $\begin{array}{l}\text { Missing cases treated as: no losses to follow- } \\
\text { up }\end{array}$ \\
\hline France & $\begin{array}{l}\text { EUROCARE-3: Electronic availability of } \\
\text { EUROCARE } 3 \text { data: a tool for further } \\
\text { analysis (P Roazzi Annals of Oncology } 14 \\
150-155,2003 .) \\
\text { National death causes database/Available } \\
\text { Eurostat Databse }\end{array}$ & ICD-10 C18. & $15+$ & & $\begin{array}{l}\text { Population: } 3 \text { regional } \\
\text { registries: Calvados, } \\
\text { Côte d'Or, Bas-Rhin, } \\
\text { representing } 2,162.000 \\
\text { persons, i.e. } 3.8 \% \text { of } \\
\text { French population in } \\
\text { 1990. Generalisable to } \\
\text { nation. }\end{array}$ & $\begin{array}{l}\text { ICSS } \\
\text { population }\end{array}$ & $\begin{array}{l}\text { French network of cancer registries survival } \\
\text { data fro } 1988 \text { to } 1997 \text { follow-up to } 2002 \text { will be } \\
\text { published soon }\end{array}$ \\
\hline Germany & Saarland Cancer Registry & $\begin{array}{l}\text { ICD-9 153, } \\
154\end{array}$ & $15-89$ & & $\begin{array}{l}\text { Population: Data refer } \\
\text { to the region of } \\
\text { Saarland, thus it is not } \\
\text { representative for all } \\
\text { Germany }\end{array}$ & & $\begin{array}{l}\text { Date of diagnosis in the years 1993-1997, } \\
\text { Follow-up for deaths was to December } 31 \text {, } \\
2000 \text {. }\end{array}$ \\
\hline Iceland & Icelandic Cancer Registry & $\begin{array}{l}\text { ICD-10 C18, } \\
\text { C19, C20 }\end{array}$ & & 1955-1995 & Yes & & Missing cases treated as: non-survivors \\
\hline Ireland & NCRI. Irish National Cancer Registry & $\begin{array}{l}\text { ICD-10 C18, } \\
\text { C19, C20 }\end{array}$ & $15-99$ & & & $\begin{array}{l}\text { Eurocare } 3 \\
\text { standard }\end{array}$ & $\begin{array}{l}\text { Missing cases treated as: follow up is passive, } \\
\text { by means of matching registered cases to }\end{array}$ \\
\hline
\end{tabular}


DELSA/HEA/WD/HWP(2007)4

\begin{tabular}{|c|c|c|c|c|c|c|c|}
\hline Country & Source & $\begin{array}{l}\text { Diagnosis } \\
\text { Code }\end{array}$ & $\begin{array}{l}\text { Age groups } \\
\text { included }\end{array}$ & $\begin{array}{l}\text { Additional } \\
\text { years }\end{array}$ & $\begin{array}{l}\text { Is this a national } \\
\text { registry? }\end{array}$ & $\begin{array}{l}\text { Reference } \\
\text { population }\end{array}$ & Comments \\
\hline & & & & & & $\begin{array}{l}\text { patient } \\
\text { populations }\end{array}$ & $\begin{array}{l}\text { death certificates. Currently matching } \\
\text { completed to } 31 \text { December } 2003 \text { (censoring } \\
\text { date) and patients not matched by this date } \\
\text { (known to have died by this date) are } \\
\text { assumed to be alive }\end{array}$ \\
\hline Italy & Registro Nazionale Tumori & $\begin{array}{l}\text { ICD-10 C18, } \\
\text { C19, C20. }\end{array}$ & $14+$ & & Yes & & Missing cases treated as: dropped out \\
\hline Japan & $\begin{array}{l}\text { Tsukuma H, Ajiki W, loka A, Oshima A, } \\
\text { and Research Group of Population-Based } \\
\text { Cancer Registry of Japan, Survival of } \\
\text { cancer patients diagnosed in 1993-96: } \\
\text { collaborative study of population-based } \\
\text { cancer registries in Japan, Japanese } \\
\text { Journal of Clinical Oncology, 36: 602-607, } \\
2006\end{array}$ & $\begin{array}{l}\text { ICD-9 } \\
153,154\end{array}$ & & & & & \\
\hline Korea & $\begin{array}{l}\text { Korea Central Cancer Registry } \\
\text { Source of mortality data: Annual Report on } \\
\text { the Cause of Death Statistics. Korea } \\
\text { National Statistical Office, 2006. }\end{array}$ & $\begin{array}{l}\text { ICD-10 C18, } \\
\text { C19, C20. }\end{array}$ & & & Yes & & $\begin{array}{l}\text { Since our cancer incidence DB is under review } \\
\text { of the International Agency for Research on } \\
\text { Cancer (IARC), statistical figures may change } \\
\text { after the completion of the review. } \\
\text { Missing cases treated as: dropped out }\end{array}$ \\
\hline Netherlands & $\begin{array}{l}\text { National Institute of Public Health and the } \\
\text { Environment. } \\
\text { Source of Mortality data: Statline. } \\
\text { Voorbrug: Statistics Netherlands, } 2006 \\
\text { Website } \\
\text { http://statline.cbs.nl/StatWeb/Start.asp?!p= } \\
\text { Search/Search\&LA=EN\&DM=SLEN. }\end{array}$ & $\begin{array}{l}\text { ICD-10 C18- } \\
20 .\end{array}$ & All & & $\begin{array}{l}\text { No. In the Netherlands, } \\
\text { the National Cancer } \\
\text { Registry of the } \\
\text { Association of } \\
\text { Comprehensive Cancer } \\
\text { Centres (CCC) delivers } \\
\text { incidence rates. } \\
\text { However, survival rates } \\
\text { can not be calculated } \\
\text { for the whole country. } \\
\text { At this moment, three } \\
\text { Comprehensive Cancer } \\
\text { Centres have data on } \\
\text { cancer survival. In a } \\
\text { few years, all CCC's } \\
\text { will deliver data on } \\
\text { cancer survival. Data } \\
\text { therefore cover over }\end{array}$ & & $\begin{array}{l}\text { Missing cases treated as: the CCC's obtain } \\
\text { data of persons in the population who died. } \\
\text { These data are obtained from the Municipal } \\
\text { Basis Registry, which is to a large extent } \\
\text { complete. Patients, of whom no death report is } \\
\text { obtained, are still alive. Emigrants are } \\
\text { censored. Overall, almost } 100 \% \text { of follow-up is } \\
\text { complete. }\end{array}$ \\
\hline
\end{tabular}


DELSA/HEA/WD/HWP(2007)4

\begin{tabular}{|c|c|c|c|c|c|c|c|}
\hline Country & Source & $\begin{array}{l}\text { Diagnosis } \\
\text { Code }\end{array}$ & $\begin{array}{l}\text { Age groups } \\
\text { included }\end{array}$ & $\begin{array}{l}\text { Additional } \\
\text { years }\end{array}$ & $\begin{array}{l}\text { Is this a national } \\
\text { registry? }\end{array}$ & $\begin{array}{l}\text { Reference } \\
\text { population }\end{array}$ & Comments \\
\hline & & & & & $\begin{array}{l}40 \% \text { of Dutch } \\
\text { population. Data have } \\
\text { been weighted by the } \\
\text { incidence of the CCC } \\
\text { into the total incidence } \\
\text { of the country. }\end{array}$ & & \\
\hline $\begin{array}{l}\text { New } \\
\text { Zealand }\end{array}$ & $\begin{array}{l}\text { New Zealand Cancer Registry (NZCR). } \\
\text { Source of mortality data: NZHIS Mortality } \\
\text { data collection 1998-2006 }\end{array}$ & $\begin{array}{l}\text { ICD-10-AM } \\
\text { (Australian } \\
\text { Modification) } \\
\text { C18-C20 } \\
\text { (excluding } \\
\text { anus and } \\
\text { anal canal). }\end{array}$ & & 1994-2006 & Yes & $\begin{array}{l}\text { OECD } \\
\text { standard } \\
\text { population } \\
1980 \text { (only for } \\
\text { relative rates) }\end{array}$ & $\begin{array}{l}\text { DCO (Death certificate only) cases were } \\
\text { excluded } \\
\text { Missing cases treated as: passive follow-up, } \\
\text { no losses }\end{array}$ \\
\hline Norway & $\begin{array}{llll}\text { Cancer } & \text { Registry } & \text { of } & \text { Norway }\end{array}$ & $\begin{array}{l}\text { ICD-10 C18, } \\
\text { C19, C20. }\end{array}$ & & & Yes & & Missing cases treated as: dropped out \\
\hline $\begin{array}{l}\text { Slovak } \\
\text { Republic }\end{array}$ & National cancer registry & $\begin{array}{l}\text { ICD-10 C18, } \\
\text { C19, C20. }\end{array}$ & & Since 1970 & Yes & $\begin{array}{l}\text { standard } \\
\text { European } \\
\text { population }\end{array}$ & $\begin{array}{l}\text { Missing cases treated as: returned to the } \\
\text { reporting units for correction, therefore we do } \\
\text { not have missing cases. }\end{array}$ \\
\hline Sweden & The Swedish Cancer Register & $\begin{array}{ll}\text { ICD-9 } & 153, \\
154\end{array}$ & & $1961-2002$ & Yes & & $\begin{array}{l}\text { Missing cases treated as: censored in a } \\
\text { survival analyses (actuarial method) }\end{array}$ \\
\hline $\begin{array}{l}\text { United } \\
\text { Kingdom }\end{array}$ & $\begin{array}{l}\text { Office for National Statistics/Department of } \\
\text { Health }\end{array}$ & $\begin{array}{l}\text { ICD-9 } 153, \\
154\end{array}$ & $15-99$ & & $\begin{array}{l}\text { Population: Data for } \\
\text { England. The age } \\
\text { profile of the England \& } \\
\text { Wales cancer } \\
\text { population is nothing } \\
\text { like the OECD } \\
\text { population. For } \\
\text { instance, } 51 \% \text { of the } \\
\text { adult OECD population } \\
\text { is age } 15-39 \text {, but only } \\
1.3 \% \text { of colorectal } \\
\text { cancer cases are in this } \\
\text { age group. }\end{array}$ & OECD 1980 & \\
\hline $\begin{array}{l}\text { United } \\
\text { States }\end{array}$ & $\begin{array}{l}\text { Surveillance Epidemiology and End } \\
\text { Results (SEER) Program } \\
\text { (www.seer.cancer.gov) SEER*Stat }\end{array}$ & & & & Generalisable to nation & OECD 1980 & \\
\hline
\end{tabular}


DELSA/HEA/WD/HWP(2007)4

\begin{tabular}{|c|c|c|c|c|c|c|c|}
\hline \multirow[b]{2}{*}{ Country } & \multirow[b]{2}{*}{ Source } & \multirow[b]{2}{*}{$\begin{array}{c}\text { Diagnosis } \\
\text { Code }\end{array}$} & \multirow[b]{2}{*}{$\begin{array}{c}\text { Age groups } \\
\text { included }\end{array}$} & \multirow[b]{2}{*}{$\begin{array}{l}\text { Additional } \\
\text { years }\end{array}$} & \multirow[b]{2}{*}{$\begin{array}{l}\text { Is this a national } \\
\text { registry? }\end{array}$} & \multicolumn{2}{|c|}{ Poforanco | } \\
\hline & & & & & & $\begin{array}{l}\text { Reference } \\
\text { population }\end{array}$ & Comments \\
\hline \multicolumn{8}{|c|}{ Non-OECD EU countries } \\
\hline Country & Source & $\begin{array}{l}\text { Diagnosis } \\
\text { Code }\end{array}$ & $\begin{array}{l}\text { Age groups } \\
\text { included }\end{array}$ & $\begin{array}{l}\text { Additional } \\
\text { years }\end{array}$ & $\begin{array}{l}\text { Is this a national } \\
\text { registry? }\end{array}$ & $\begin{array}{l}\text { Reference } \\
\text { population }\end{array}$ & Comments \\
\hline Latvia & $\begin{array}{l}\text { Cancer Registry } \\
\text { Source of mortality data: National Death } \\
\text { Causes Database. }\end{array}$ & $\begin{array}{l}\text { ICD-10 C18, } \\
\text { C19, C20. }\end{array}$ & & & Yes & & $\begin{array}{l}\text { Missing cases treated as: In the calculation } \\
\text { from "missing cases" } 1 / 2 \text { treated as survivors } \\
\text { and other } 1 / 2 \text { as non-survivors. }\end{array}$ \\
\hline Malta & $\begin{array}{l}\text { Malta National Cancer Registry: } \\
\text { http://www.sahha.gov.mt/pages.aspx?pag } \\
\text { e=91 } \\
\text { Mortality Source: Malta National Cancer } \\
\text { Registry: } \\
\text { http://www.sahha.gov.mt/pages.aspx?pag } \\
\text { e=91 }\end{array}$ & & & & & & $\begin{array}{l}\text { Mortality figures are age-standardised using } \\
\text { European Standard Population }\end{array}$ \\
\hline
\end{tabular}


Incidence of vaccine preventable diseases (Pertussis, measles, hepatitis B)

Operational Definition

Numerator: Number of reported cases.

Denominator: Total 100000 population.

Table 10. Incidence of vaccine preventable diseases (Pertussis, measles, hepatitis B)

\begin{tabular}{|c|c|c|c|c|}
\hline Country & Data year & Hepatitis B & Measles & Pertussis \\
\hline \multirow[t]{7}{*}{ Australia } & 2006 & 1.80 & 0.70 & 65.80 \\
\hline & 2005 & 1.10 & 0.00 & 51.80 \\
\hline & 2004 & 1.40 & 0.20 & 42.50 \\
\hline & 2003 & 1.70 & 0.50 & 25.70 \\
\hline & 2002 & 2.10 & 0.20 & 28.30 \\
\hline & 2001 & 2.10 & 0.70 & 48.00 \\
\hline & 2000 & 2.10 & 0.60 & 31.30 \\
\hline \multirow[t]{2}{*}{ Austria } & 2005 & 7.00 & 0.10 & 1.60 \\
\hline & 2000 & 3.30 & 0.00 & 1.40 \\
\hline \multirow[t]{2}{*}{ Canada } & 2004 & 2.70 & 0.03 & 8.79 \\
\hline & 1999 & 4.20 & 0.10 & 20.00 \\
\hline Czech Republic & 2005 & 3.50 & 0.00 & 5.40 \\
\hline \multirow[t]{2}{*}{ Denmark } & 2005 & 0.50 & 0.04 & 2.40 \\
\hline & 2004 & 0.80 & 0.00 & 4.22 \\
\hline \multirow[t]{2}{*}{ Finland } & 2005 & 0.63 & 0.02 & 10.54 \\
\hline & 2001 & 2.40 & 0.02 & 6.10 \\
\hline \multirow[t]{2}{*}{ France } & 2003 & $<1$ & 7.00 & NA \\
\hline & 2001 & 5.00 & 12.00 & NA \\
\hline \multirow[t]{2}{*}{ Germany } & 2004 & 1.50 & 0.15 & $\overline{N A}$ \\
\hline & 2001 & 2.90 & 7.30 & NA \\
\hline \multirow[t]{3}{*}{ Iceland $^{1}$} & 2005 & NA & 0.00 & 2.00 \\
\hline & 2004 & NA & 0.00 & 0.30 \\
\hline & 2002 & NA & 0.00 & 4.00 \\
\hline Ireland & 2005 & 2.29 & NA & NA \\
\hline \multirow[t]{3}{*}{ Italy } & 2004 & 2.02 & 1.18 & 2.31 \\
\hline & 2003 & 2.24 & 20.90 & 2.23 \\
\hline & 2002 & 2.35 & 31.15 & 4.45 \\
\hline \multirow[t]{4}{*}{ Japan } & 2005 & NA & 4.7 & 8.7 \\
\hline & 2004 & NA & 9.45 & 10.23 \\
\hline & 2003 & NA & 60.90 & 11.00 \\
\hline & 2000 & NA & 27.00 & 1.41 \\
\hline \multirow[t]{3}{*}{ Mexico } & 2005 & 0.59 & 0.01 & 0.33 \\
\hline & 2004 & 0.65 & 0.06 & 0.13 \\
\hline & 2001 & NA & 0.00 & 0.20 \\
\hline \multirow[t]{9}{*}{ Netherlands } & 2005 & 1.83 & 0.02 & 40.04 \\
\hline & 2004 & 1.80 & 0.07 & 55.95 \\
\hline & 2003 & 2.03 & 0.02 & 16.65 \\
\hline & 2002 & 1.70 & 0.02 & 36.39 \\
\hline & 2001 & 1.30 & 0.11 & 43.54 \\
\hline & 2000 & NA & 6.40 & 30.36 \\
\hline & 1999 & NA & 14.98 & 39.71 \\
\hline & 1998 & NA & 0.06 & 14.34 \\
\hline & 1997 & NA & 0.13 & 25.28 \\
\hline
\end{tabular}




\begin{tabular}{|l|c|c|c|c|}
\hline \multicolumn{5}{|c|}{ Cont. } \\
\hline \multirow{2}{*}{ Country } & Data year & Hepatitis B & Measles & Pertussis \\
\hline New Zealand & 2005 & 1.60 & 0.50 & 72.80 \\
& 2000 & 2.10 & 1.80 & NA \\
\hline Norway ${ }^{2}$ & 2005 & 3.10 & 0.00 & 120.00 \\
& 2004 & 4.10 & 0.20 & 170.00 \\
& 2001 & 4.50 & 0.10 & 57.30 \\
\hline Poland & 2005 & 1.70 & 0.03 & 5.04 \\
\hline Portugal & 2005 & 0.92 & 0.07 & 0.74 \\
& 2003 & 1.13 & 0.07 & 0.03 \\
& 2001 & 2.03 & 0.26 & 0.01 \\
\hline Slovak Republic & 2005 & 1.90 & 0.00 & 0.30 \\
& 2004 & 2.06 & 0.04 & NA \\
\hline Spain & 2004 & 1.93 & 0.07 & 1.34 \\
& 2001 & 2.24 & 0.37 & 2.32 \\
\hline Sweden & 2005 & 2.40 & 0.10 & 15.10 \\
& 2004 & 2.90 & 0.10 & 17.50 \\
& 2002 & 19.40 & 0.10 & 15.10 \\
\hline Switzerland ${ }^{3}$ & 2005 & 2.00 & 2.00 & 56.00 \\
& 2001 & 2.10 & 10.00 & 80.00 \\
\hline United Kingdom & 2004 & 2.29 & 4.44 & 0.95 \\
& 2003 & 2.18 & 4.71 & 0.77 \\
\hline United States & 2004 & 2.10 & 0.00 & 8.90 \\
& 2003 & 2.61 & 0.02 & 4.04 \\
& 2000 & 6.30 & 0.00 & 2.70 \\
\hline
\end{tabular}

\begin{tabular}{|l|c|c|c|c|}
\hline \multicolumn{5}{|c|}{ Non-OECD EU countries } \\
\hline \multicolumn{1}{|c|}{ Country } & Data year & Hepatitis B & Measles & Pertussis \\
\hline Cyprus ${ }^{4}$ & 2005 & 0.80 & 0.10 & 0.80 \\
\hline Latvia & 2005 & 7.40 & 0.09 & 1.10 \\
\hline Malta & 2005 & 3.22 & 1.49 & 1.75 \\
\hline
\end{tabular}

Notes:

NA stands for "no data available".

1. 2 confirmed cases for Pertussis.

2. Pertussis' figure for 2006 is 142 per 100000 .

3. Hepatitis B for 2001 refers to 2002.

4. See footnotes 2 and 3 on page 3 . 


\section{DELSA/HEA/WD/HWP(2007)4}

Incidence of vaccine preventable diseases (Pertussis, measles, hepatitis B), sources and methods

\begin{tabular}{|c|c|c|c|c|c|c|}
\hline Country & Source & $\begin{array}{l}\text { Confirmed or } \\
\text { suspected } \\
\text { cases? }\end{array}$ & $\begin{array}{c}\text { National/regional } \\
\text { variation of } \\
\text { vaccination policies: }\end{array}$ & $\begin{array}{l}\text { Additional } \\
\text { years } \\
\text { available: }\end{array}$ & $\begin{array}{l}\text { Reporting } \\
\text { Mandated? }\end{array}$ & Comments \\
\hline Australia & $\begin{array}{l}\text { National Notifiable Diseases Surveillance } \\
\text { System, www.health.gov.au/cda, viewed } 8 \\
\text { December } 2006\end{array}$ & & & & & \\
\hline Austria & Federal Ministry of Health and Women & & & & & $\begin{array}{l}\text { No differentiation of Hepatitis B (acute), } \\
\text { preliminary data. }\end{array}$ \\
\hline Canada & Public Health Agency of Canada & $\begin{array}{l}\text { Confirmed } \\
\text { cases only }\end{array}$ & $\begin{array}{l}\text { The National Advisory } \\
\text { Committee has a national } \\
\text { guideline for Measles and } \\
\text { Pertussis. HBV vaccine is } \\
\text { now given routinely in most } \\
\text { provinces and territories to } \\
\text { young adolescents or to } \\
\text { both infants and young } \\
\text { adolescents. Hepatitis B } \\
\text { vaccine is also } \\
\text { recommended for certain } \\
\text { groups at higher risk of } \\
\text { infection with HBV. }\end{array}$ & & & \\
\hline Cyprus* $^{*}$ & $\begin{array}{l}\text { Medical and Public Health Services, } \\
\text { Ministry of Health }\end{array}$ & & & Since 1980 & & \\
\hline $\begin{array}{l}\text { Czech } \\
\text { Republic }\end{array}$ & National Institute of Public Health & & $\begin{array}{l}\text { Hepatitis is not part of the } \\
\text { general vaccination } \\
\text { programme. }\end{array}$ & $\begin{array}{l}\text { Hepatitis B } \\
\text { since } 1980\end{array}$ & & $\begin{array}{l}\text { Preliminary results. Pertussis (A37-ICD- } \\
\text { 10); Measles (B05-ICD-10); Hepatitis B } \\
\text { (B16-ICD-10). }\end{array}$ \\
\hline Denmark & Statens Serum Institut & $\begin{array}{l}\text { Suspected and } \\
\text { confirmed cases }\end{array}$ & & $\begin{array}{l}\text { Acute hep. B } \\
\text { from 1985, } \\
\text { measles from } \\
1994 \text { and } \\
\text { Pertussis } \\
\text { since } 1980\end{array}$ & & $\begin{array}{l}\text { Pertussis is only reportable by law if the } \\
\text { person in-question is under } 2 \text { years old. } \\
\text { Hepatitis is not part of the general } \\
\text { vaccination programme }\end{array}$ \\
\hline Finland & $\begin{array}{l}\text { National Infection Register at the National } \\
\text { Institute of Public Health }\end{array}$ & $\begin{array}{l}\text { In Finland the } \\
\text { national }\end{array}$ & & & & \\
\hline
\end{tabular}

\footnotetext{
${ }^{*}$ See footnotes 2 and 3 on page 3.
} 
DELSA/HEA/WD/HWP(2007)4

\begin{tabular}{|c|c|c|c|c|c|c|}
\hline Country & Source & $\begin{array}{l}\text { Confirmed or } \\
\text { suspected } \\
\text { cases? }\end{array}$ & $\begin{array}{c}\text { National/regional } \\
\text { variation of } \\
\text { vaccination policies: }\end{array}$ & $\begin{array}{l}\text { Additional } \\
\text { years } \\
\text { available: }\end{array}$ & $\begin{array}{c}\text { Reporting } \\
\text { Mandated? }\end{array}$ & Comments \\
\hline & & $\begin{array}{l}\text { infection register } \\
\text { registers } \\
\text { confirmed cases } \\
\text { only, suspected } \\
\text { cases are not } \\
\text { registered. }\end{array}$ & & & & \\
\hline France & $\begin{array}{l}\text { Réseau sentinelle for measles. disease } \\
\text { surveillance national institut (in VS) for } \\
\text { hepatitis B }\end{array}$ & & & & $\begin{array}{l}\text { Yes for } \\
\text { hepatitis, No } \\
\text { for others. For } \\
\text { measles is } \\
\text { mandatory } \\
\text { since } 2005 \text {. }\end{array}$ & $\begin{array}{l}\text { Hepatitis B: } 158 \text { cases notified in the } \\
\text { whole country from } 1 / 03 / 2003 \text { to } \\
1 / 03 / 2004 \text {. No incidence data for } \\
\text { Pertussis; RENACOQ, which is a } \\
\text { hospitals network created in } 1996, \\
\text { including } 44 \text { hospitals with paediatrics } \\
\text { units, monitors Pertussis cases. Its goal } \\
\text { is to describe epidemiological } \\
\text { characteristics of children Pertussis, from } \\
\text { a hospital point of view, and not to } \\
\text { calculate an incidence rate. Réseau } \\
\text { sentinelle is a General practitioners } \\
\text { network, on voluntary basis, which } \\
\text { collects and analyses epidemiological } \\
\text { data on GP activity. GPs are included on } \\
\text { a voluntary basis. Data concern } \\
\text { transmissible diseases that are frequent } \\
\text { in general practice: Influenza, acute } \\
\text { diarrhoea, measles, mumps, varicella, } \\
\text { hepatitis A, B, C. But now, as cases have } \\
\text { became rarer and rarer, exhaustive } \\
\text { cases notification is required for hepatitis } \\
\text { B (since march 2003) and measles } \\
\text { (2005). Incidence of measles and } \\
\text { hepatitis B decreases as vaccination rate } \\
\text { increases. However, hepatitis B } \\
\text { vaccination rate does not increase as } \\
\text { much as measles rate. }\end{array}$ \\
\hline Germany & Robert Koch Institute & & & & $\begin{array}{l}\text { Yes for } \\
\text { measles and } \\
\text { Hep B. No for } \\
\text { Pertussis. }\end{array}$ & $\begin{array}{l}\text { Mandatory reporting is regulated on a } \\
\text { federal state but not on a national level, } \\
\text { hence data representative on a national } \\
\text { level cannot be provided. }\end{array}$ \\
\hline Iceland & Directorate of Health & & & & & \\
\hline
\end{tabular}


DELSA/HEA/WD/HWP(2007)4

\begin{tabular}{|c|c|c|c|c|c|c|}
\hline Country & Source & $\begin{array}{l}\text { Confirmed or } \\
\text { suspected } \\
\text { cases? }\end{array}$ & $\begin{array}{c}\text { National/regional } \\
\text { variation of } \\
\text { vaccination policies: }\end{array}$ & $\begin{array}{l}\text { Additional } \\
\text { years } \\
\text { available: }\end{array}$ & $\begin{array}{c}\text { Reporting } \\
\text { Mandated? }\end{array}$ & Comments \\
\hline Ireland & $\begin{array}{l}\text { HSE Health Protection Surveillance } \\
\text { Centre }\end{array}$ & $\begin{array}{l}\text { The results are } \\
\text { based on } \\
\text { suspected } \\
\text { cases. }\end{array}$ & & & & $\begin{array}{l}\text { Notified cases: Pertussis: } 90 \text {, } \\
\text { measles: } 327 \text {, hep B: } 701 . \text { for Hep B, see } \\
\text { paper submission }\end{array}$ \\
\hline Italy & Ministry of Health & $\begin{array}{l}\text { The results are } \\
\text { based on } \\
\text { suspected and } \\
\text { confirmed } \\
\text { cases. }\end{array}$ & & $2002-2003$. & Yes & Missing cases treated as: Dropped out \\
\hline Japan & $\begin{array}{l}\text { Calculated from "Taniguchi K. Evaluation } \\
\text { and Improvement for effective infectious } \\
\text { disease surveillance system. Health, } \\
\text { Labour \& Welfare research grant report } \\
\text { 2006. P65 and P68. }\end{array}$ & & & & & \\
\hline Mexico & $\begin{array}{l}\text { Ministry of Health (Secretaría de Salud), } \\
\text { Mexico, SUIVE: Sistema Único de } \\
\text { Vigilancia Epidemiológica, Indicator } \\
\text { coverage: total population, national level. }\end{array}$ & & & & & \\
\hline Netherlands & $\begin{array}{l}\text { National Institute for Public Health and the } \\
\text { Environment, Centre for Infectious } \\
\text { Disease Epidemiology, 2006. Website: } \\
\text { http://www.rivm.nl/isis/ggd/openbaar/tot/tb } \\
\text { lj.html. } \\
\end{array}$ & $\begin{array}{l}\text { The results are } \\
\text { based on } \\
\text { confirmed } \\
\text { cases. }\end{array}$ & & & & $\begin{array}{l}\text { Pertussis: every period of } 2 \text { to } 3 \text { years an } \\
\text { epidemiologic increase occurs. Country- } \\
\text { wide immunisation for children against } \\
\text { was introduced in } 1952 \text {. Measles: every } \\
\text { period of } 5 \text { to } 7 \text { years an epidemiologic } \\
\text { increase occurs. Country-wide } \\
\text { immunisation for children against } \\
\text { measles was introduced in } 1976 \text {. } \\
\text { Hepatitis B: In } 20031,877 \text { cases of } \\
\text { hepatitis B were reported (afterwards } \\
\text { corrected to } 1,900 \text { ) of which } 319 \text { acute } \\
\text { and } 1,445 \text { chronic cases (Koedijk et al., } \\
2005) \text {. Of } 113 \text { cases the type of hepatitis } \\
\text { B infection was unknown. In } 2002 \text { the } \\
\text { number reported cases of acute hepatitis } \\
\text { B was } 265 \text {. In November } 2002 \\
\text { vaccination for groups with high risk } \\
\text { behaviour was introduced. The Municipal } \\
\text { Public Health Services performs the } \\
\text { tracing of these groups: homo- and }\end{array}$ \\
\hline
\end{tabular}


DELSA/HEA/WD/HWP(2007)4

\begin{tabular}{|c|c|c|c|c|c|c|}
\hline Country & Source & $\begin{array}{c}\text { Confirmed or } \\
\text { suspected } \\
\text { cases? }\end{array}$ & $\begin{array}{c}\text { National/regional } \\
\text { variation of } \\
\text { vaccination policies: }\end{array}$ & $\begin{array}{l}\text { Additional } \\
\text { years } \\
\text { available: }\end{array}$ & $\begin{array}{l}\text { Reporting } \\
\text { Mandated? }\end{array}$ & Comments \\
\hline & & & & & & $\begin{array}{l}\text { bisexual men, prostitutes, heterosexual } \\
\text { persons with a health care consult } \\
\text { related to a sexual transmitting disease, } \\
\text { and drug users. Since } 2000 \text { employers in } \\
\text { health care have the obligation to give } \\
\text { their health care workers (including } \\
\text { students) the opportunity to have a } \\
\text { vaccination. In January } 2003 \text {, hepatitis B } \\
\text { vaccination was added to the National } \\
\text { Immunisation Programme (NIP) for } \\
\text { children born to parents from middle or } \\
\text { high endemic countries (birth cohort } 1^{\text {st }} \\
\text { January } 2003 \text { onwards). Vaccination } \\
\text { against hepatitis B for children born to } \\
\text { mothers tested positive for HBsAg was } \\
\text { introduced in } 1989 \text {. In January } 2006 \\
\text { vaccination at birth was added to the NIP } \\
\text { for these children. }\end{array}$ \\
\hline $\begin{array}{l}\text { New } \\
\text { Zealand }\end{array}$ & $\begin{array}{l}\text { Numerator: ESR Annual Surveillance } \\
\text { Report 2005. Denominator: Statistics New } \\
\text { Zealand, usually resident population from } \\
\text { the } 2001 \text { Census. }\end{array}$ & & & & & $\begin{array}{l}\text { Figures are based on Notifications } 2000 \\
\text { (some laboratory confirmed for Measles). } \\
\text { Hepatitis B was the only vaccine } \\
\text { preventable disease to show a significant } \\
\text { increase in notification rate compared } \\
\text { with } 2004 \text { ( } 1.0 \text { per } 100000) \text {. Measles: } \\
\text { one outbreak in } 19 \text { cases. Pertussis: } \\
\text { during the latter part of } 2004 \text { and the first } \\
\text { three months of } 2005 \text { New Zealand } \\
\text { experienced an epidemic of Pertussis. By } \\
\text { comparison, the annual rate was } 15.7 \\
\text { per } 100000 \text {. }\end{array}$ \\
\hline Norway & $\begin{array}{l}\text { MSIS: The Norwegian System for } \\
\text { Notification of Infectious Diseases }\end{array}$ & $\begin{array}{l}\text { The results are } \\
\text { based on } \\
\text { confirmed } \\
\text { cases. }\end{array}$ & & & & \\
\hline Poland & $\begin{array}{l}\text { National Institute of Hygiene, Department } \\
\text { of Epidemiology }\end{array}$ & $\begin{array}{l}\text { According to EU } \\
\text { definitions the } \\
\text { reported cases } \\
\text { are suspected } \\
\text { and/or probable }\end{array}$ & & & & \\
\hline
\end{tabular}


DELSA/HEA/WD/HWP(2007)4

\begin{tabular}{|c|c|c|c|c|c|c|}
\hline Country & Source & $\begin{array}{l}\text { Confirmed or } \\
\text { suspected } \\
\text { cases? }\end{array}$ & $\begin{array}{c}\text { National/regional } \\
\text { variation of } \\
\text { vaccination policies: }\end{array}$ & $\begin{array}{c}\text { Additional } \\
\text { years } \\
\text { available: }\end{array}$ & $\begin{array}{l}\text { Reporting } \\
\text { Mandated? }\end{array}$ & Comments \\
\hline & & $\begin{array}{l}\text { and/or } \\
\text { confirmed for } \\
\text { Pertussis and } \\
\text { Measles, } \\
\text { probable and/or } \\
\text { confirmed for } \\
\text { Hepatitis B. } \\
\end{array}$ & & & & \\
\hline Portugal & DGS & & & & & \\
\hline $\begin{array}{l}\text { Slovak } \\
\text { Republic }\end{array}$ & Public Health Office & & & & & \\
\hline Spain & $\begin{array}{l}\text { Ministry of Health and Consumer Affairs } \\
\text { (Ministerio de Sanidad y } \\
\text { Consumo.Instituto de Salud Carlos IIII) }\end{array}$ & & & $\begin{array}{l}\text { Pertussis } \\
\text { since 1983, } \\
\text { Measles } \\
\text { suspected } \\
\text { cases since } \\
1994, \text { and } \\
\text { registered } \\
\text { cases since } \\
2002, \text { acute } \\
\text { Hepatitis B } \\
\text { since } 1995 .\end{array}$ & & \\
\hline Sweden & $\begin{array}{l}\text { Swedish Institute for Infectious Disease } \\
\text { Control. }\end{array}$ & & & & & \\
\hline Switzerland & Notifications/sentinel surveillance & & & & & \\
\hline $\begin{array}{l}\text { United } \\
\text { Kingdom }\end{array}$ & Health Protection Agency & & & & Yes & $\begin{array}{l}\text { Measles in UK is not always lab } \\
\text { confirmed, notifications are reported on } \\
\text { clinical suspicion, if lab test shows it is } \\
\text { not measles then it is supposed to be } \\
\text { denotified, but this often doesn't happen, } \\
\text { so measles incidence is likely to be an } \\
\text { overestimate. }\end{array}$ \\
\hline $\begin{array}{l}\text { United } \\
\text { States }\end{array}$ & $\begin{array}{l}\text { CDC's Notifiable Disease Surveillance } \\
\text { System. }\end{array}$ & & & & & $\begin{array}{l}\text { As of March } 2004 \text {, all } 50 \text { states allow } \\
\text { vaccination exemptions for medical } \\
\text { reasons; } 48 \text { states allow exemptions for } \\
\text { religious reasons; and } 20 \text { states allow } \\
\text { exemptions for philosophical reasons. } \\
\text { Please comment on potential effects of } \\
\text { national/regional vaccination policies on } \\
\text { this indicator, if applicable: it is the }\end{array}$ \\
\hline
\end{tabular}


DELSA/HEA/WD/HWP(2007)4

\begin{tabular}{|l|l|c|c|c|c|c|}
\hline Country & Source & $\begin{array}{c}\text { Confirmed or } \\
\text { suspected } \\
\text { cases? }\end{array}$ & $\begin{array}{c}\text { National/regional } \\
\text { variation of } \\
\text { vaccination policies: }\end{array}$ & $\begin{array}{c}\text { Additional } \\
\text { years } \\
\text { available: }\end{array}$ & $\begin{array}{c}\text { Reporting } \\
\text { Mandated? }\end{array}$ & \\
\hline & & & & $\begin{array}{l}\text { Cosponsibility of individual states to } \\
\text { determine which vaccines are required } \\
\text { by law. Currently all } 50 \text { states have } \\
\text { school immunisation laws, although there } \\
\text { are differences in what may be required } \\
\text { in different states. }\end{array}$ \\
\hline
\end{tabular}

\begin{tabular}{|c|c|c|c|c|c|c|}
\hline \multicolumn{7}{|c|}{ Non-OECD EU countries } \\
\hline Country & Source & $\begin{array}{l}\text { Confirmed or } \\
\text { suspected } \\
\text { cases? }\end{array}$ & $\begin{array}{c}\text { National/regional } \\
\text { variation of } \\
\text { vaccination policies: }\end{array}$ & $\begin{array}{l}\text { Additional } \\
\text { years } \\
\text { available: }\end{array}$ & $\begin{array}{l}\text { Reporting } \\
\text { Mandated?: }\end{array}$ & Comments \\
\hline Latvia & Public Health State Agency & $\begin{array}{l}\text { The results are } \\
\text { based on } \\
\text { suspected and } \\
\text { confirmed } \\
\text { cases. }\end{array}$ & & & & \\
\hline
\end{tabular}


DELSA/HEA/WD/HWP(2007)4

Coverage for basic vaccination programme, age 2, (Pertussis, measles, hepatitis B)

Operational Definition

Numerator: Number of children who are fully immunised at age 2 for basic vaccination programme.

Denominator: Number of children age 2 years. 
Table 11. Coverage for basic vaccination programme, age 2, (Pertussis, measles, hepatitis B)

\begin{tabular}{|c|c|c|c|c|c|}
\hline Country & Data year & Overall \% & Hepatitis B \% & $\begin{array}{c}\text { Measles (MMR) } \\
\%\end{array}$ & $\begin{array}{c}\text { Pertussis (DPT) } \\
\%\end{array}$ \\
\hline \multirow[t]{3}{*}{ Australia } & 2006 & 92.2 & 95.8 & 93.9 & 95.1 \\
\hline & 2005 & 92.1 & 95.9 & 93.8 & 95.2 \\
\hline & 2004 & 91.7 & NA & 93.6 & 95.0 \\
\hline Austria & 2004 & $\mathrm{NA}$ & 86.0 & 57.0 & 87.0 \\
\hline \multirow[t]{2}{*}{ Canada } & 2004 & NA & $\mathrm{NA}$ & 94.0 & 74.0 \\
\hline & 2002 & NA & NA & 94.5 & 75.2 \\
\hline Czech Republic & 2005 & NA & 98.5 & 96.6 & 98.7 \\
\hline \multirow[t]{2}{*}{ Denmark } & 2005 & NA & $\mathrm{NA}$ & 95.5 & 95.6 \\
\hline & 2001 & NA & NA & 96.0 & 95.0 \\
\hline \multirow[t]{2}{*}{ Finland $^{\top}$} & 2005 & NA & NA & 97.0 & 97.0 \\
\hline & 2002 & 93.3 & NA & 96.6 & 95.6 \\
\hline \multirow[t]{2}{*}{ France } & 2004 & $\mathrm{NA}$ & 30.0 & 87.1 & 90.3 \\
\hline & 2001 & NA & 28.0 & 84.6 & 87.9 \\
\hline Germany & 1999 & NA & 73.0 & 73.0 & 89.0 \\
\hline \multirow[t]{4}{*}{ Iceland } & 2005 & NA & $\mathrm{NA}$ & 94.0 & 97.0 \\
\hline & 2004 & NA & NA & 94.0 & NA \\
\hline & 2003 & NA & NA & 93.0 & 97.0 \\
\hline & 1999 & $\mathrm{NA}$ & NA & 92.0 & 98.0 \\
\hline Ireland & 2004 & 90.0 & NA & 83.0 & 91.0 \\
\hline \multirow[t]{4}{*}{ Italy } & 2005 & NA & 95.7 & 87.3 & 96.2 \\
\hline & 2004 & NA & 96.3 & 85.7 & 94.0 \\
\hline & 2003 & NA & 95.3 & 83.9 & 95.8 \\
\hline & 2002 & NA & 95.7 & 81.1 & 94.2 \\
\hline \multirow[t]{4}{*}{ Japan } & 2005 & $\overline{N A}$ & $\mathrm{NA}$ & 95.6 & 97.7 \\
\hline & 2004 & 63.9 & NA & 85.8 & 82.7 \\
\hline & 2003 & NA & NA & 88.8 & 85.0 \\
\hline & 2001 & NA & NA & 80.9 & 83.3 \\
\hline \multirow[t]{2}{*}{ Mexico } & 2005 & 99.3 & 99.4 & 98.5 & 99.4 \\
\hline & 2004 & NA & 99.5 & 98.6 & 99.5 \\
\hline \multirow[t]{2}{*}{ Netherlands $^{2}$} & 2005 & NA & $\mathrm{NA}$ & 96.3 & 97.8 \\
\hline & 2001 & NA & NA & 95.6 & 95.3 \\
\hline New Zealand & 2005 & 77.4 & 86.5 & 82.0 & 88.6 \\
\hline \multirow[t]{3}{*}{ Norway } & 2005 & 89.0 & $\mathrm{NA}$ & 89.0 & 91.0 \\
\hline & 2004 & NA & NA & 88.0 & 91.0 \\
\hline & 2001 & NA & NA & 90.0 & 91.0 \\
\hline Poland & 2005 & NA & 99.8 & 98.2 & 98.8 \\
\hline \multirow[t]{3}{*}{ Portugal } & 2004 & NA & 96.0 & 94.8 & 97.8 \\
\hline & 2003 & NA & 96.6 & 95.6 & 96.8 \\
\hline & 2001 & NA & 94.7 & 87.7 & 98.1 \\
\hline \multirow[t]{2}{*}{ Slovak Republic } & 2005 & $\mathrm{NA}$ & 99.2 & 98.4 & $98.7-99.2$ \\
\hline & 2004 & 98.0 & NA & NA & NA \\
\hline \multirow[t]{3}{*}{ Spain $^{3}$} & 2005 & NA & 96.1 & 96.8 & 96.2 \\
\hline & 2004 & NA & 98.2 & 97.3 & 97.0 \\
\hline & 2001 & NA & NA & 97.3 & 96.0 \\
\hline \multirow[t]{3}{*}{ Sweden } & 2005 & 95.6 & 2.6 & 95.4 & 98.7 \\
\hline & 2004 & NA & NA & 94.5 & 98.4 \\
\hline & 2002 & NA & NA & 88.4 & 98.8 \\
\hline \multirow[t]{2}{*}{ Switzerland } & 2005 & 73.0 & NA & 86.0 & 96.0 \\
\hline & $2000-2002$ & 76.0 & NA & NA & NA \\
\hline \multirow[t]{2}{*}{ United Kingdom } & $2004-2005$ & $\mathrm{NA}$ & NA & 81 & 94 \\
\hline & 2003-2004 & NA & NA & 80.0 & 93.0 \\
\hline \multirow[t]{3}{*}{ United States } & 2004 & 80.9 & 92.4 & 93.0 & 85.5 \\
\hline & 2003 & 79.4 & 92.4 & 93.0 & 96.0 \\
\hline & 2001 & 78.6 & 93.0 & 91.4 & 94.3 \\
\hline
\end{tabular}


DELSA/HEA/WD/HWP(2007)4

\begin{tabular}{|l|c|c|c|c|c|}
\hline \multicolumn{1}{|c|}{ Non-OECD EU countries } \\
\hline Country & Data year & Overall \% & Hepatitis B \% & $\begin{array}{c}\text { Measles (MMR) } \\
\%\end{array}$ & $\begin{array}{c}\text { Pertussis (DPT) } \\
\%\end{array}$ \\
\hline Cyprus $^{5}$ & 2006 & 86.0 & 93.2 & 39.5 & 96.8 \\
\hline Latvia $^{\text {Malta }}{ }^{4}$ & 2005 & NA & 98.1 & 95.0 & 95.6 \\
\hline
\end{tabular}

NA stands for "no data available".

1. Overall rate refers to 2002.

2. DTP rate comprises complete first series offered at 2, 3 and 4 months, assessed at 24-36 months.

3. Children under 1 year old.

4. Rates for Hepatitis B and Pertussis (DPT) refer to percentage of children up to their first birthday, whereas rate for Measles (MMR) refers to percentage children up to their second birthday.

5. See footnotes 2 and 3 on page 3 . 
DELSA/HEA/WD/HWP(2007)4

Coverage for basic vaccination programme, age 2, (Pertussis, measles, hepatitis B), sources and methods

\begin{tabular}{|c|c|c|c|c|}
\hline Country & Source & $\begin{array}{c}\text { Description of the basic immunisation } \\
\text { program: }\end{array}$ & $\begin{array}{l}\text { Additional } \\
\text { years } \\
\text { available: }\end{array}$ & Comments \\
\hline Australia & $\begin{array}{l}\text { Australian Childhood Immunisation } \\
\text { Register (ACIR) }\end{array}$ & & & $\begin{array}{l}\text { Data are for birth cohorts } 1 \text { July to } 30 \text { September } \\
2003 \text {, assessed } 31 \text { December } 2005\end{array}$ \\
\hline Austria & Federal Ministry of Health and Women & $\begin{array}{l}\text { Mumps, Measles, Rubella, Diphtheria, Pertussis, } \\
\text { Tetanus, Poliomyelitis, Hepatitis B, Heamophilus } \\
\text { influenza - no compulsory vaccination in Austria. }\end{array}$ & & \\
\hline Canada & $\begin{array}{l}\text { Public Health Agency of Canada ( } 2004 \\
\text { National Immunization Coverage Survey } \\
\text { (NICS)) }\end{array}$ & & & \\
\hline $\begin{array}{l}\text { Czech } \\
\text { Republic }\end{array}$ & $\begin{array}{l}\text { Department of Chief Public Health Officer } \\
\text { of the CR }\end{array}$ & $\begin{array}{l}\text { DTP: 1st dose - 9th-12th week, 2nd dose - 13th-16th } \\
\text { week, 3rd dose - 17th-20th week, 4th dose - 18th-20th } \\
\text { month; MMR: 1st dose - 15th month, 2nd dose }-21 \text { st- } \\
\text { 25th month; Hepatitis B: } 3 \text { doses in the interval 0-1-6 } \\
\text { months, 1st dose - in the first months of live, usually } \\
\text { 1st and 2nd doses are given in conjunction with DTP- } \\
\text { HiB. }\end{array}$ & & Data for DPT vaccination rate relates to 4 doses \\
\hline Finland & National Public Health Institute & & & $\begin{array}{l}\text { The vaccination coverage study is done in Finland in } \\
\text { systematic regular intervals. The vaccination } \\
\text { coverage studies are done by the National Public } \\
\text { Health Institute. The over all vaccination figure } \\
\text { coverage is from the previous study, from year } \\
\text { 2002. The other figures from the following study. }\end{array}$ \\
\hline France & $\begin{array}{l}\text { French Ministry of Health, DREES } \\
\text { (Statistics Department) }\end{array}$ & $\begin{array}{l}\text { Program Description: Vaccination against Diphtheria - } \\
\text { tetanus, Poliomyelitis, BCG is mandatory. Vaccinations } \\
\text { against Pertussis, Haemophilus influenzae b, Hepatitis } \\
\text { B, Measles, Mumps, Rubella are not mandatory, but } \\
\text { strongly recommended. MMR booster has been } \\
\text { recommended at age. } 2 \text { since } 2005 \text {. These }\end{array}$ & & $\begin{array}{l}\text { Vaccination rates for Diphtheria - tetanus and } \\
\text { Poliomyelitis are calculated for a full vaccination } \\
\text { (including } 3 \text { doses + booster). So are calculated } \\
\text { Pertussis and H. Influenzae vaccination rates. }\end{array}$ \\
\hline
\end{tabular}


DELSA/HEA/WD/HWP(2007)4

\begin{tabular}{|c|c|c|c|c|}
\hline Country & Source & $\begin{array}{c}\text { Description of the basic immunisation } \\
\text { program: }\end{array}$ & $\begin{array}{l}\text { Additional } \\
\text { years } \\
\text { available: }\end{array}$ & Comments \\
\hline & & $\begin{array}{l}\text { percentages concern } 24 \text { months children (+/- } 1 \text { month). } \\
4 \text { doses Diphtheria, Tetanus, Poliomyelitis, HiB; } 3 \\
\text { doses Hepatitis B; } 1 \text { dose Measles, Mumps, Rubella }\end{array}$ & & \\
\hline Germany & $\begin{array}{l}\text { Lauberau et al. (2001) } \\
\text { Durchimpfungsraten bei Kindern in } \\
\text { Deutschland 1999, Monatsschr } \\
\text { Kinderheikd 149, } 367-372\end{array}$ & $\begin{array}{l}\text { Program Description: Diphtheria (D/d), acellular } \\
\text { Pertussis (aP), Tetanus (T), Haemophilus influenzae } \\
\text { Type b (Hib), Hepatitis B (HB), Poliomyelitis (IPV), } \\
\text { measles, mumps, rubella (MMR), are recommended by } \\
\text { Standing Committee on Vaccination (STIKO) by } 2 \\
\text { years. }\end{array}$ & & $\begin{array}{l}\text { Regional non-representative survey data not } \\
\text { generalisable to national level. } \\
\text { According to recommendations of the Standing } \\
\text { Committee on Vaccination (STIKO) at the Robert } \\
\text { Koch Institute (www.rki.de) basic childhood } \\
\text { immunisation up to the age of } 2 \text { years includes } \\
\text { vaccinations against the following diseases: } \\
\text { diphtheria (D), tetanus (T), Pertussis (aP), } \\
\text { poliomyelitis (IPV), haemophilus influenzae (HiB), } \\
\text { hepatitis B (HB), measles, mumps, rubella (MMR } \\
\text { combination vaccine), and varizella zoster virus } \\
\text { (VZV) infections. The recommended time schedule } \\
\text { is: DTaP, Hib, IPV, HB at ages } 2,3,4 \text {, and } 11-14 \\
\text { months; MMR at ages } 11-14 \text { and } 15-23 \text { months; } \\
\text { VZV at ages } 11-14 \text { months.Approximately } 90 \% \text { of } \\
\text { vaccinations are administered by privately practising } \\
\text { physicians in Germany. In general, vaccination is } \\
\text { covered by statutory health insurance when } \\
\text { recommended by the STIKO. Small sample size, not } \\
\text { representative. }\end{array}$ \\
\hline Iceland & Directorate of Health & & & \\
\hline Ireland & $\begin{array}{l}\text { HSE, Health Protection Surveillance } \\
\text { Centre }\end{array}$ & $\begin{array}{l}\text { Program Description: DTP is tracked separately for } \\
\text { diphtheria, Pertussis and tetanus ( } 3 \text { doses of each } \\
\text { antigen by age } 2 \text { ). }\end{array}$ & & \\
\hline Italy & Ministry of Health & $\begin{array}{l}\text { The basic immunisation program in Italy comprises } \\
\text { mandatory and recommended vaccines. The } \\
\text { mandatory ones are: diphtheria, tetanus, polio and } \\
\text { hepatitis B; the recommended are measles, mumps, } \\
\text { rubella, MMR, Pertussis, haemophilus influenzae type } \\
\text { B. The immunisation schedules have been updated } \\
\text { with the Ministerial decrees of } 7 \text { April } 1999 \text { (concerning } \\
\text { the passage from an all OPV immunisation schedule to } \\
\text { a sequential schedule) and of } 18 \text { June } 2002 \text {; (this last } \\
\text { concerning the shift from a sequential polio schedule to } \\
\text { an all IPV immunisation schedule). The shots are given } \\
\text { at } 3,5,11 \text { months for DTP, Hib, polio, hepatitis B, and at }\end{array}$ & $2000-2005$ & \\
\hline
\end{tabular}


DELSA/HEA/WD/HWP(2007)4

\begin{tabular}{|c|c|c|c|c|}
\hline Country & Source & $\begin{array}{c}\text { Description of the basic immunisation } \\
\text { program: }\end{array}$ & $\begin{array}{l}\text { Additional } \\
\text { years } \\
\text { available: }\end{array}$ & Comments \\
\hline & & $\begin{array}{l}12-15 \text { months for MMR. A booster dose of polio } \\
\text { vaccine is scheduled during the third year of age. The } \\
\text { vaccines are administered by the personnel of the local } \\
\text { health units. }\end{array}$ & & \\
\hline Japan & $\begin{array}{l}\text { Administrative reports of the Community } \\
\text { and Elderly Health. The data covers only } \\
\text { vaccination provided by municipal } \\
\text { governments and may be underestimated } \\
\text { because privately-paid vaccination is not } \\
\text { included (privately-paid vaccination is } \\
\text { small, though). }\end{array}$ & $\begin{array}{l}\text { DPT } 3 \text { times }+1 \text { booster shot by } 7.5 \text { years old, Polio } 2 \\
\text { times, Measles and Rubella by } 7.5 \text { years old. }\end{array}$ & & $\begin{array}{l}\text { Data are not available with unique identifier. So the } \\
\text { lowest rate (\% people who completed DPT, } 63.9 \%) \\
\text { was adopted as an overall rate. \% of Polio } 85.7 \% \text {, } \\
\text { Measles } 85.8 \% \text {, Rubella } 78 \% \text {. }\end{array}$ \\
\hline Mexico & $\begin{array}{l}\text { Vaccination Programme. Ministry of Health } \\
\text { (Secretaría de Salud), Mexico. }\end{array}$ & $\begin{array}{l}\text { Includes: DPT, Mumps, Measles, Rubella, anti- } \\
\text { Haemophilus influenzae b, Poliomyelitis, Hepatitis B, } \\
\text { BCG (anti-TB). }\end{array}$ & & \\
\hline Netherlands & $\begin{array}{l}\text { Abbink F, Oomen PJ, Zwakhals SLN, } \\
\text { Melker HE de, Ambler-Huiskes A. } \\
\text { Vaccinatietoestand Nederland per } 1 \\
\text { januari 2005. [Immunisation coverage in } \\
\text { the Netherlands as at } 1 \text { January 2005]. } \\
\text { RIVM rapport } 210021005 / 2006 \text {. Bilthoven: } \\
\text { RIVM, 2006. Developments in 2006. RIVM } \\
\text { report xxxx/2007. Bilthoven: The } \\
\text { Netherlands. In preparation }\end{array}$ & $\begin{array}{l}\text { Vaccination schedule: DTPA and IPV at } 2,3,4 \text { and } 11 \\
\text { months; MMR at } 14 \text { months; Hepatitis B (risk groups } \\
\text { only) at birth, } 2,3,4 \text { and } 11 \text { months. At the age of } 4 \\
\text { DTPA and IPV are offered, and at the age of } 9 \text { DT, IPV } \\
\text { and MMR. Also included in the immunisation } \\
\text { programme are vaccinations against pneumococcal } \\
\text { disease (2, } 3,4,11 \text { months), Haemophilus influenzae } \\
\text { type b (2, 3, } 4,11 \text { months), and infections with } \\
\text { meningococci serogroup C (14 months). Every child is } \\
\text { offered vaccination from birth to } 13 \text { years on a } \\
\text { voluntary basis and free of charge. The Ministry of } \\
\text { Public Health, Welfare and Sports decides on the } \\
\text { vaccination policy, the Netherlands Vaccine Institute is } \\
\text { responsible for delivering all vaccines and the National } \\
\text { Institute of Health and the Environment (RIVM) advises } \\
\text { the Ministry. The execution of the Dutch National } \\
\text { Immunisation Programme is coordinated by the Centre } \\
\text { for Infectious Disease Control (Clb) of the RIVM. For } \\
\text { children up to the age of } 4 \text { years the programme is } \\
\text { usually implemented by the network of Maternal and } \\
\text { Child Health Clinics, for school-aged children by the } \\
\text { Municipal Public Health Services. } \\
\text { The Regional Vaccination Administration Centres } \\
\text { (since April } 2007 \text { forming a part of the Clb of the RIVM) }\end{array}$ & & $\begin{array}{l}\text { Data about the immunisation rate for hepatitis B in } \\
\text { children are not available yet, because this } \\
\text { vaccination is introduced recently (2003). Hepatitis } \\
\text { B vaccination within the framework of the basic } \\
\text { immunisation programme is administered to risk } \\
\text { group only (children of whom at least one parent } \\
\text { was born in a country where hepatitis B is } \\
\text { moderately or highly endemic and children of whom } \\
\text { the mother is hepatitis B carrier). }\end{array}$ \\
\hline
\end{tabular}


DELSA/HEA/WD/HWP(2007)4

\begin{tabular}{|c|c|c|c|c|}
\hline Country & Source & $\begin{array}{l}\text { Description of the basic immunisation } \\
\text { program: }\end{array}$ & $\begin{array}{l}\text { Additional } \\
\text { years } \\
\text { available: }\end{array}$ & Comments \\
\hline & & $\begin{array}{l}\text { maintain a database of vaccination records for each } \\
\text { child living in the region. These centres take care of } \\
\text { updating the database by processing records from the } \\
\text { Municipal Basis Registry about birth, deaths and } \\
\text { removal. }\end{array}$ & & \\
\hline $\begin{array}{l}\text { New } \\
\text { Zealand }\end{array}$ & $\begin{array}{l}\text { National Immunisation Coverage Survey } \\
2005\end{array}$ & $\begin{array}{l}\text { National Immunisation Schedule effective from } 1 \\
\text { February 2006: } 6 \text { weeks - DTaP-IPV (diphtheria, } \\
\text { tetanus, acellular Pertussis, inactivated polio vaccine), } \\
\text { Hib-Hep B (haemophilus influenzae type b, hepatitis } \\
\text { B); } 3 \text { months - DTaP-IPV, Hib-Hep B; } 5 \text { months - } \\
\text { DTaP-IPV, Hep B; } 5 \text { months - Hib, MMR (measles, } \\
\text { mumps, rubella); } 4 \text { years - DTaP-IPV, MMR; } 11 \text { years } \\
\text { - dTap-IPV* (adult diphtheria, tetanus, adult acellular } \\
\text { Pertussis, inactivated polio vaccine until the end of } \\
2007 \text { for those who have not previously had four } \\
\text { doses) Special programme: } 6 \text { weeks - MeNZB } \\
\text { (meningococcal B); } 3 \text { months - MeNZB; } 5 \text { months - } \\
\text { MeNZB; } 10 \text { months - MeNZB (those who received the } \\
3^{\text {rd }} \text { dose between } 5-6 \text { months of age, otherwise } \\
\text { minimum } 4 \text { months after the } 3^{\text {rd }} \text { dose). }\end{array}$ & & $\begin{array}{l}\text { Data from the National Immunisation Register (NIR) } \\
\text { will be available in late } 2007 \text {. }\end{array}$ \\
\hline Norway & $\begin{array}{l}\text { SYSVAK: National electronic vaccination } \\
\text { register in Norway }\end{array}$ & $\begin{array}{l}\text { The Norwegian Childhood Vaccination Schedule: } 3 \\
\text { months: DTP, Hib, Polio (IPV), Pneumococcal } \\
\text { conjugate vaccine (Pn7v). } 5 \text { months DTP, Hib, Polio } \\
\text { (IPV), Pneumococcal conjugate vaccine (Pn7v). } 12 \\
\text { months DTP, Hib, Polio (IPV), Pneumococcal } \\
\text { conjugate vaccine (Pn7v). } 15 \text { months MMR. 7-8 years } \\
\text { DTP, Polio (IPV). 11-12 years dT (for children born } \\
\text { before 1998). 12-13 years MMR. 13-15 years BCG. 15- } \\
16 \text { years Polio (IPV), dT (for children born in 1998 or } \\
\text { later).. }\end{array}$ & & \\
\hline Poland & $\begin{array}{l}\text { National Institute of Hygiene, Department } \\
\text { of Epidemiology }\end{array}$ & $\begin{array}{l}\text { Tuberculosis, Hepatitis B, Tetanus, Diphteria, } \\
\text { Pertussis, Poliomyelitits, Measles, Rubella, Mumps }\end{array}$ & $1980+$ & \\
\hline Portugal & $\begin{array}{l}\text { "Direcção Geral de Saúde" - Health } \\
\text { Ministry. }\end{array}$ & $\begin{array}{l}\text { Till } 12 \text { months: DTP (Diphtheria, Tetanus, Pertussis); } \\
\text { Polio; BCG; HIB; Hepatitis B. Between } 12 \text { and } 23 \\
\text { months: VASPR (Measles, Mumps, Rubella). }\end{array}$ & $1985+$ & Last available data - 2004. \\
\hline $\begin{array}{l}\text { Slovak } \\
\text { Republic }\end{array}$ & Public Health Office & $\begin{array}{l}\text { Immunisation program in the Slovak rep is realised } \\
\text { since } 1954 \text { by low. IP included vaccination against } 10 \\
\text { antigens: TB, poliomyelitis, VHB, diphtheria, Pertussis, } \\
\text { Tetanus, Haemophilius influenza type B, Measles, }\end{array}$ & & \\
\hline
\end{tabular}


DELSA/HEA/WD/HWP(2007)4

\begin{tabular}{|c|c|c|c|c|}
\hline Country & Source & $\begin{array}{c}\text { Description of the basic immunisation } \\
\text { program: }\end{array}$ & $\begin{array}{l}\text { Additional } \\
\text { years } \\
\text { available: }\end{array}$ & Comments \\
\hline & & mumps, rubella..; & & \\
\hline Spain & $\begin{array}{l}\text { Ministry of Health and Consumer Affairs. } \\
\text { (Ministerio de Sanidad y Consumo. } \\
\text { Dirección General de Salud Pública) }\end{array}$ & $\begin{array}{l}\text { http//www.msc.es/ciudadanos/proteccionSalud/infancia } \\
\text { /docs/c2006.Pdf }\end{array}$ & & $\begin{array}{l}\text { Vaccinations by private sector are not included. } \\
\text { Vaccinations rate - Percentage of infants reaching } \\
\text { their first birthday who have been fully immunised }\end{array}$ \\
\hline Sweden & $\begin{array}{l}\text { Swedish Institute for Infectious Disease } \\
\text { Control }\end{array}$ & $\begin{array}{l}\text { Nationwide reports in January } 2006 \text { from all child } \\
\text { health centres in Sweden regarding vaccination that } \\
\text { passed their } 2 \text { year during the preceding calendar year } \\
\text { (Sweden Data Year } 2004-2005 \text { - birth cohort born in } \\
2003 \text { vaccinated during the period from birth up to } \\
\text { January 2006). }\end{array}$ & & \\
\hline Switzerland & Monitoring in 9 cantons & & & \\
\hline $\begin{array}{l}\text { United } \\
\text { Kingdom }\end{array}$ & Department of Health & $\begin{array}{l}\text { Program Description: It is not possible to give an } \\
\text { aggregated proportion of children who have had all the } \\
\text { standard vaccinations, so we have reported a rate for } \\
\text { each of the standard vaccination programs. } \\
\text { Description of the basic immunisation program: } \\
\text { DTaP/IPV/Hib is a primary immunisation given to } \\
\text { babies when they are } 2,3 \text { and } 4 \text { months old. The } \\
\text { DTaP/IPV/Hib vaccine protects against five different } \\
\text { diseases: diphtheria (D); tetanus (T); Pertussis. } \\
\text { Meningitis C is a primary immunisation given to babies } \\
\text { when they are } 2,3 \text { and } 4 \text { months old. }\end{array}$ & & Data are for England only. \\
\hline $\begin{array}{l}\text { United } \\
\text { States }\end{array}$ & CDC National Immunization Survey & $\begin{array}{l}\text { It is the responsibility of individual states to determine } \\
\text { which vaccines are required by law, although most look } \\
\text { to the schedule of recommended childhood vaccines } \\
\text { established and updated each year by the Committee } \\
\text { on Infectious Diseases of the American Academy of } \\
\text { Pediatrics. The Advisory Committee on Immunization } \\
\text { Practices of the Centers for Disease Control and } \\
\text { Prevention, and the American Academy of Family } \\
\text { Physicians. Currently all } 50 \text { states have school } \\
\text { immunization laws, although there are differences in } \\
\text { what may be required in different states. } \\
\text { Deviation from age at measurement (2 years)? 19-35 } \\
\text { months. }\end{array}$ & & $\begin{array}{l}\text { As of March 2004, all } 50 \text { states allow vaccination } \\
\text { exemptions for medical reasons; } 48 \text { states allow } \\
\text { exemptions for religious reasons; and } 20 \text { states } \\
\text { allow exemptions for philosophical reasons. }\end{array}$ \\
\hline
\end{tabular}


DELSA/HEA/WD/HWP(2007)4

\begin{tabular}{|c|c|c|c|c|}
\hline \multicolumn{5}{|c|}{ Non-OECD EU countries } \\
\hline Country & Source & $\begin{array}{l}\text { Description of the basic immunisation } \\
\text { program: }\end{array}$ & $\begin{array}{l}\text { Additional } \\
\text { years } \\
\text { available: }\end{array}$ & Comments \\
\hline Cyprus* & $\begin{array}{l}\text { Medical and Public Health Services, } \\
\text { Ministry of Health }\end{array}$ & $\begin{array}{l}\text { The childhood immunisation programme in Cyprus* is } \\
\text { set at national level by the Ministry of Health. In the } \\
\text { public sector immunisation is carried out by the health } \\
\text { visitors in the child health centres and the school } \\
\text { health services under the guidance of the public health } \\
\text { doctors while in the private sector vaccines are given } \\
\text { by the paediatricians. In the public sector the following } \\
\text { vaccinations are given free of charge to all the citizens } \\
\text { regardless of their socioeconomic status: DPT, } \\
\text { Poliomyelitis (IPV/OPV), MMR, Hepatitis B and } \\
\text { Heamophilus Influenza type b (Hib). } \\
\text { Deviation from age at measurement (2 years): Yes. }\end{array}$ & & $\begin{array}{l}\text { Due to lack of statistical information from the private } \\
\text { sector, the Ministry of Health and particularly the } \\
\text { Medical and Public Health Services perform a } 3 \\
\text { year survey for the immunisation coverage at } \\
\text { national level in children } 17-24 \text { months. This survey } \\
\text { is carried out in accordance with the } \\
\text { recommendations and a relevant protocol of the } \\
\text { World Health Organisation to determine vaccine } \\
\text { coverage and the degree of correct timing of the first } \\
\text { and subsequent vaccination administration } \\
\text { according to the WHO guidelines. According to the } \\
\text { results of the last survey which was carried out in } \\
\text { May } 2006 \text { among children } 17-24 \text { months, the } \\
\text { coverage for DTP3 was } 96 \% \text {, for OPV3 } 96,5 \% \text {, for } \\
\text { DTP and OPV3 was } 96,5 \% \text {, for DTP3, OPV } 3 \text { and } \\
\text { MMR was } 86,2 \% \text { and for Hepatitis B } 93,2 \% \text {. The } \\
\text { given values are estimations rely on those } \\
\text { percentages. }\end{array}$ \\
\hline Latvia & Public Health State Agency & $\begin{array}{l}\text { The Basic immunisation program contains children's } \\
\text { vaccination against Tuberculosis, Tetanus, Diphtheria, } \\
\text { Whooping cough, Poliomyelitis, Measles, Rubella, } \\
\text { Mumps, Hepatitis B, B type Haemophilus influenzae. }\end{array}$ & & $\begin{array}{l}\text { Measles vaccination at the age } 16-23 \text { months; DT - } \\
95,6 \text { and P 95,6; Hepatitis B - } 1 \text { year. }\end{array}$ \\
\hline Malta & WHO-HFA: http://data.euro.who.int/hfadb/ & & & \\
\hline
\end{tabular}

\footnotetext{
${ }^{*}$ See footnotes 2 and 3 on page 3 .
} 
Asthma mortality rate, ages 5-39

\section{Operational Definition}

Numerator: Number of people dying from asthma as a primary cause, age 5-39

[Asthma diagnostic codes ICD-9-493 or ICD-10-J45, J 46].

Denominator: 100000 people age 5-39.

Table 12. Asthma mortality rate, ages 5-39

\begin{tabular}{|c|c|c|c|c|c|c|c|c|}
\hline \multirow{2}{*}{ Country } & \multicolumn{8}{|c|}{ Asthma mortality per 100000 people } \\
\hline & 1998 & 1999 & 2000 & 2001 & 2002 & 2003 & 2004 & 2005 \\
\hline Australia & & & 0.61 & 0.56 & 0.41 & 0.37 & 0.40 & \\
\hline Austria & & & 0.10 & & & & & 0.11 \\
\hline Canada & & & & & 0.11 & 0.20 & & \\
\hline Czech Republic & & & & & & & & 0.10 \\
\hline Denmark & & & & 0.41 & & & & 0.17 \\
\hline Finland & & & & 0.26 & & 0.00 & 0.04 & 0.00 \\
\hline France & & & 0.92 & 0.30 & & & & \\
\hline Germany & & & & & & 0.16 & & \\
\hline Iceland & & 0.00 & & & 0.00 & & & 0.00 \\
\hline Ireland & & & & & & 0.38 & & \\
\hline Italy & & & 0.13 & 0.14 & 0.11 & & & \\
\hline Japan & & & & 0.28 & & 0.27 & 0.26 & 0.19 \\
\hline Korea & & & & & & & & 0.09 \\
\hline Mexico & & & & & 0.32 & & & 0.30 \\
\hline Netherlands & & & 0.15 & & 0.13 & & & 0.11 \\
\hline New Zealand & & & 0.80 & & & 0.35 & & \\
\hline Norway & & & 0.05 & & & 0.18 & 0.05 & \\
\hline Poland & & & & & & & 0.08 & \\
\hline Portugal & & & 0.10 & & 0.16 & & 0.08 & \\
\hline Slovak Republic & & & & & & & 0.11 & \\
\hline Spain & 0.19 & & & & & & 0.17 & \\
\hline Sweden & & & 0.07 & & & 0.12 & & \\
\hline Switzerland & & 0.20 & & & & 0.00 & & \\
\hline United Kingdom & & & & & 0.58 & 0.40 & 0.49 & \\
\hline United States & & & & & 0.47 & 0.33 & & \\
\hline
\end{tabular}

\begin{tabular}{|c|c|c|c|c|c|c|c|c|}
\hline \multicolumn{9}{|c|}{ Non-OECD EU countries } \\
\hline \multirow[t]{2}{*}{ Country } & \multicolumn{8}{|c|}{ Asthma mortality per 100000 people } \\
\hline & 1998 & 1999 & 2000 & 2001 & 2002 & 2003 & 2004 & 2005 \\
\hline Cyprus & & & & & & & & 0.25 \\
\hline Latvia & & & & & & & & 0.37 \\
\hline Malta & & & & & & & & 0.00 \\
\hline
\end{tabular}

Note:

1. See footnotes 2 and 3 on page 3 . 
DELSA/HEA/WD/HWP(2007)4

Asthma mortality rate, ages 5-39, sources and methods

\begin{tabular}{|c|c|c|c|c|c|}
\hline Country & Source & $\begin{array}{l}\text { Diagnoses } \\
\text { code(s) }\end{array}$ & $\begin{array}{l}\text { Additional } \\
\text { years } \\
\text { available: }\end{array}$ & $\begin{array}{l}\text { Reference } \\
\text { population }\end{array}$ & Comments \\
\hline Australia & AlHW Mortality Database & $\begin{array}{l}\text { ICD-10 J45, } \\
\text { J46. }\end{array}$ & & 1980 OECD & \\
\hline Austria & Statistics Austria. & $\begin{array}{l}\text { ICD-10 J45, } \\
\text { J46. }\end{array}$ & & & \\
\hline Canada & $\begin{array}{l}\text { Canadian Vital Statistics Mortality } \\
\text { Database }\end{array}$ & $\begin{array}{l}\text { ICD-10 J45, } \\
\text { J46. }\end{array}$ & & $\begin{array}{l}1991 \text { Canada } \\
\text { Census }\end{array}$ & \\
\hline $\begin{array}{l}\text { Czech } \\
\text { Republic }\end{array}$ & $\begin{array}{l}\text { Vital Statistics (Czech Statistical } \\
\text { Office) }\end{array}$ & $\begin{array}{l}\text { ICD-10 J45, } \\
\text { J46. }\end{array}$ & & & \\
\hline Denmark & National Causes of Death Register & $\begin{array}{l}\text { ICD-10 J45, } \\
\text { J46. }\end{array}$ & & & $\begin{array}{l}\text { Denmark wishes to make sure that the possibility of underreporting for } \\
\text { this indicator is recognised. The underreporting makes comparisons } \\
\text { difficult and could be misleading. }\end{array}$ \\
\hline Finland & $\begin{array}{l}\text { National Cause of Death Register, } \\
\text { Statistical Institution of Finland }\end{array}$ & $\begin{array}{l}\text { ICD-10 J45, } \\
\text { J46. }\end{array}$ & & & \\
\hline France & $\begin{array}{l}\text { Numerator: national exhaustive } \\
\text { mortality data (centre for } \\
\text { epidemiology of medical causes of } \\
\text { deaths - INSERM-CépiDc) for } \\
\text { numerator, INSEE (Statistics } \\
\text { National Institute) for population) }\end{array}$ & $\begin{array}{l}\text { ICD-10 J45, } \\
46\end{array}$ & & & $1999(0.8376)$ \\
\hline Germany & $\begin{array}{l}\text { Todesursachenstatistik (causes of } \\
\text { death statistics) }\end{array}$ & $\begin{array}{l}\text { ICD-10 J45, } \\
\text { J46 }\end{array}$ & & & Value for ICD-10 J40-J47 is: 0.28. \\
\hline Iceland & Directorate of Health & & & & \\
\hline Ireland & PHIS & ICD-9-493 & & $\begin{array}{l}\text { PHIS } \\
\text { Population } \\
\text { Data }\end{array}$ & \\
\hline Italy & $\begin{array}{l}\text { Italian mortality database collected } \\
\text { by ISTAT and processed by Istituto } \\
\text { Superiore di Sanità. }\end{array}$ & ICD-9 493. & & $\begin{array}{l}1991 \text { Italian } \\
\text { Census }\end{array}$ & \\
\hline Japan & vital statistics & $\begin{array}{l}\text { ICD-10 J45, } \\
\text { J46. }\end{array}$ & & & \\
\hline Korea & $\begin{array}{l}\text { Annual Report on the Cause of } \\
\text { Death Statistics. Korea National } \\
\text { Statistical Office, } 2006 .\end{array}$ & $\begin{array}{l}\text { ICD-10 J45, } \\
\text { J46. }\end{array}$ & & & $\begin{array}{l}\text { 1. Number of people dying from asthma as a primary cause ( } \mathrm{J} 45, \mathrm{~J} 46) \text {, } \\
\text { age } 5-39: 242 \text {. Chronic lower respiratory disease mortality }(\mathrm{J} 40-47) \\
\text { was } 0.16 \text {. }\end{array}$ \\
\hline Mexico & Mortality figures: Mortalidad 2005, & ICD-10 J45, & & & \\
\hline
\end{tabular}


DELSA/HEA/WD/HWP(2007)4

\begin{tabular}{|c|c|c|c|c|c|}
\hline Country & Source & $\begin{array}{c}\text { Diagnoses } \\
\text { code(s) }\end{array}$ & $\begin{array}{l}\text { Additional } \\
\text { years } \\
\text { available: }\end{array}$ & $\begin{array}{l}\text { Reference } \\
\text { population }\end{array}$ & Comments \\
\hline & $\begin{array}{l}\text { Instituto Nacional de Estadística, } \\
\text { Geografía e Informática y Dirección } \\
\text { General de Información en Salud, } \\
\text { Secretaría de Salud. Population } \\
\text { figures: Instituto Nacional de } \\
\text { Geografía, Estadística e } \\
\text { Informática. }\end{array}$ & J46. & & & \\
\hline Netherlands & $\begin{array}{l}\text { National Institute for Public Health } \\
\text { and the Environment }\end{array}$ & & & OECD 1980 & \\
\hline New Zealand & $\begin{array}{l}\text { Numerator: NZHIS Mortality Data } \\
\text { Collection 2003. Denominator: } \\
\text { Statistics New Zealand, estimated } \\
\text { resident mean population year } \\
\text { ended } 31 \text { December } 2003 \text {. }\end{array}$ & $\begin{array}{l}\text { ICD-10-AM } \\
\text { (Australian } \\
\text { Modification) } \\
\text { J45, J46. }\end{array}$ & & OECD 1980 & \\
\hline Norway & Statistics Norway (SSB) & $\begin{array}{l}\text { ICD-10 J45, } \\
\text { J46. }\end{array}$ & & & \\
\hline Poland & $\begin{array}{l}\text { Data from Central Statistical Office } \\
\text { processed by National Institute of } \\
\text { Hygiene }\end{array}$ & $\begin{array}{l}\text { ICD-10 J45, } \\
\text { J46. }\end{array}$ & $1999-2003$ & $\begin{array}{l}\text { Standard } \\
\text { Population } \\
1990 \\
\end{array}$ & Figure represents crude and age-standardised rate. \\
\hline Portugal & DGS/INE & $\begin{array}{l}\text { ICD-10 J45, } \\
\text { J46. }\end{array}$ & & & $\begin{array}{l}\text { The age groups } 10-14 ; 20-24 ; 25-29 ; 30-34 \text {, do not have any registered } \\
\text { cases of death. }\end{array}$ \\
\hline $\begin{array}{l}\text { Slovak } \\
\text { Republic }\end{array}$ & Statistical Office of SR & $\begin{array}{l}\text { ICD-10 J45, } \\
\text { J46. }\end{array}$ & & $\begin{array}{l}\text { Standard } \\
\text { European } \\
\text { population }\end{array}$ & \\
\hline Spain & $\begin{array}{l}\text { Ministry of Health and Consumer } \\
\text { Affairs. (Ministerio de Sanidad y } \\
\text { Consumo. Instituto de Información } \\
\text { Sanitaria. Indicadores de salud } \\
\text { 2006). }\end{array}$ & $\begin{array}{l}\text { ICD-10 J45, } \\
\text { J46. }\end{array}$ & & & \\
\hline Sweden & $\begin{array}{l}\text { The Swedish Cause of Death } \\
\text { Register }\end{array}$ & $\begin{array}{l}\text { ICD-10 J45, } \\
\text { J46. }\end{array}$ & & $\begin{array}{l}\text { European (not } \\
\text { OECD) and } \\
\text { the Swedish } \\
\text { gave the same } \\
\text { results. }\end{array}$ & \\
\hline Switzerland & Federal Office of Statistics & ICD-9 493. & & & \\
\hline $\begin{array}{l}\text { United } \\
\text { Kingdom }\end{array}$ & $\begin{array}{l}\text { Department of Health Mortality } \\
\text { Extract } 1993-04 \text {, Office for National }\end{array}$ & $\begin{array}{l}\text { ICD-10 J45- } \\
\text { J46 }\end{array}$ & & OECD 1980 & Data are for England only \\
\hline
\end{tabular}


DELSA/HEA/WD/HWP(2007)4

\begin{tabular}{|c|l|l|l|l|l|}
\hline Country & \multicolumn{1}{|c|}{ Source } & $\begin{array}{c}\text { Diagnoses } \\
\text { code(s) }\end{array}$ & $\begin{array}{l}\text { Additional } \\
\text { years } \\
\text { available: }\end{array}$ & $\begin{array}{l}\text { Reference } \\
\text { population }\end{array}$ & \\
\hline & $\begin{array}{l}\text { Statistics; Mid-year Population } \\
\text { Estimates 1993-04, Office for } \\
\text { National Statistics; Calculations by } \\
\text { National Centre for Health } \\
\text { Outcomes Development. }\end{array}$ & & & & \\
\hline United States & $\begin{array}{l}\text { National Vital Statistics System } \\
\text { Mortality }\end{array}$ & ICD-9 493. & & OECD 1980 & Age specific rates provided \\
\hline
\end{tabular}

\begin{tabular}{|l|l|l|l|l|l|}
\hline \multicolumn{1}{|c|}{ Source } & $\begin{array}{c}\text { Diagnoses } \\
\text { code(s) }\end{array}$ & $\begin{array}{l}\text { Additional } \\
\text { years } \\
\text { available: }\end{array}$ & $\begin{array}{l}\text { Reference } \\
\text { population }\end{array}$ & Comments \\
\hline Cyprus* & Cyprus* Death Registry & $\begin{array}{l}\text { ICD-10 J45, } \\
\text { J46. }\end{array}$ & & & \\
\hline Latvia & Death Causes Database & $\begin{array}{l}\text { ICD-10 J45, } \\
\text { J46. }\end{array}$ & & & \\
\hline Malta & $\begin{array}{l}\text { National Mortality Registry, dept. of } \\
\text { Health Information, Malta }\end{array}$ & & & & \\
\hline
\end{tabular}

\footnotetext{
${ }^{*}$ See footnotes 2 and 3 on page 3 .
} 


\section{In-hospital mortality rate within 30 days of hospital admission for AMI}

\section{Operational Definition}

Numerator: Number of deaths in the hospital that occurred within 30 days of hospital admission with primary diagnosis of acute myocardial infarction (ICD-9 410 or ICD-10 I21, I22).

Denominator: Number of people hospitalised with primary diagnosis of acute myocardial infarction.

Table 13. In-hospital mortality rate within 30 days of hospital admission for AMI

\begin{tabular}{|c|c|c|c|}
\hline \multirow[b]{2}{*}{ Country } & \multicolumn{2}{|c|}{ Rate } & \multirow[b]{2}{*}{ Data year } \\
\hline & $\begin{array}{c}\text { Hospital } \\
\text { admissions- } \\
\text { based }\end{array}$ & $\begin{array}{l}\text { Unique } \\
\text { identifiers }\end{array}$ & \\
\hline \multirow[t]{2}{*}{ Australia } & 6.4 & & $2004-2005$ \\
\hline & 8.8 & & 2000-2001 \\
\hline Austria & 12.0 & & 2004 \\
\hline Canada & 9.3 & & 2004-2005 \\
\hline Czech Republic & 8.9 & & 2004 \\
\hline \multirow[t]{2}{*}{ Denmark } & & 6.4 & 2005 \\
\hline & & 6.5 & 2004 \\
\hline \multirow[t]{4}{*}{ Finland } & 11.1 & 16.2 & 2005 \\
\hline & 11.1 & 15.7 & 2004 \\
\hline & 13.0 & 18.0 & 2003 \\
\hline & 14.0 & 16.3 & 2001 \\
\hline \multirow[t]{3}{*}{ France } & 7.6 & & 2005 \\
\hline & 8.0 & & 2003 \\
\hline & 10.9 & & 2001 \\
\hline Germany & 11.9 & & 1999 \\
\hline \multirow[t]{3}{*}{ Iceland $^{2}$} & 6.4 & & 2005 \\
\hline & 6.7 & & 2004 \\
\hline & 11.6 & & 2002 \\
\hline Ireland & 10.7 & & 2003 \\
\hline \multirow[t]{3}{*}{ Italy $^{2}$} & 9.2 & & 2004 \\
\hline & 9.6 & & 2003 \\
\hline & 15.4 & & 2001 \\
\hline \multirow[t]{2}{*}{ Japan } & 10.5 & & 2005 \\
\hline & 10.3 & & 1999 \\
\hline Korea $^{1}$ & 18.6 & & 2004 \\
\hline \multirow[t]{3}{*}{ Mexico } & 24.5 & & 2005 \\
\hline & 23.1 & & 2004 \\
\hline & 14.3 & & 1999 \\
\hline
\end{tabular}




\begin{tabular}{|c|c|c|c|}
\hline \multicolumn{4}{|c|}{ cont. } \\
\hline \multirow[b]{2}{*}{ Country } & \multicolumn{2}{|c|}{ Rate } & \multirow[b]{2}{*}{ Data year } \\
\hline & $\begin{array}{c}\text { Hospital } \\
\text { admissions- } \\
\text { based }\end{array}$ & $\begin{array}{c}\text { Unique } \\
\text { identifiers }\end{array}$ & \\
\hline \multirow[t]{3}{*}{ Netherlands } & 8.4 & 9.2 & 2005 \\
\hline & 9.3 & 9.9 & 2004 \\
\hline & 11.0 & & 2001 \\
\hline \multirow[t]{2}{*}{ New Zealand } & 5.4 & 7.6 & $2005-2006$ \\
\hline & 8.9 & 10.9 & 2000-2001 \\
\hline \multirow[t]{2}{*}{ Norway } & 8.0 & & 2005 \\
\hline & 10.0 & & 2004 \\
\hline Poland & 8.0 & & 2004-2005 \\
\hline \multirow[t]{3}{*}{ Portugal } & 11.8 & & 2005 \\
\hline & 12.0 & & 2004 \\
\hline & 12.6 & & 2001 \\
\hline \multirow[t]{2}{*}{ Slovak Republic ${ }^{2,3}$} & 12.0 & & 2005 \\
\hline & 11.9 & & 2004 \\
\hline Spain & 10.3 & & 2004 \\
\hline \multirow[t]{3}{*}{ Sweden } & 8.3 & & 2005 \\
\hline & 8.5 & & 2004 \\
\hline & 10.3 & & 2001 \\
\hline \multirow[t]{2}{*}{ Switzerland $^{4}$} & 8.1 & & 2005 \\
\hline & 6.9 & & 2004 \\
\hline \multirow[t]{2}{*}{ United Kingdom $^{2}$} & 11.8 & & $2003-2004$ \\
\hline & 5.2 & & $2002-2003$ \\
\hline
\end{tabular}

\begin{tabular}{|l|c|c|c|}
\hline \multicolumn{4}{|c|}{ Non-OECD EU countries } \\
\hline Country & $\begin{array}{c}\text { Hospital } \\
\text { admissions- } \\
\text { based }\end{array}$ & $\begin{array}{c}\text { Unique } \\
\text { identifiers }\end{array}$ & Data year \\
\hline Latvia & 15.8 & & 2005 \\
\hline
\end{tabular}

Notes:

1. Data also include patients that died within 30 days after admission, out of hospital.

2. Data with a limited generalisability to national level. See sources and methods for more information.

3. Age-standardised to Standard European Population.

4. In-hospital mortality, not necessarily 30-days in-hospital mortality after admission. 
In-hospital mortality rate within 30 days of hospital admission for AMI, sources and methods

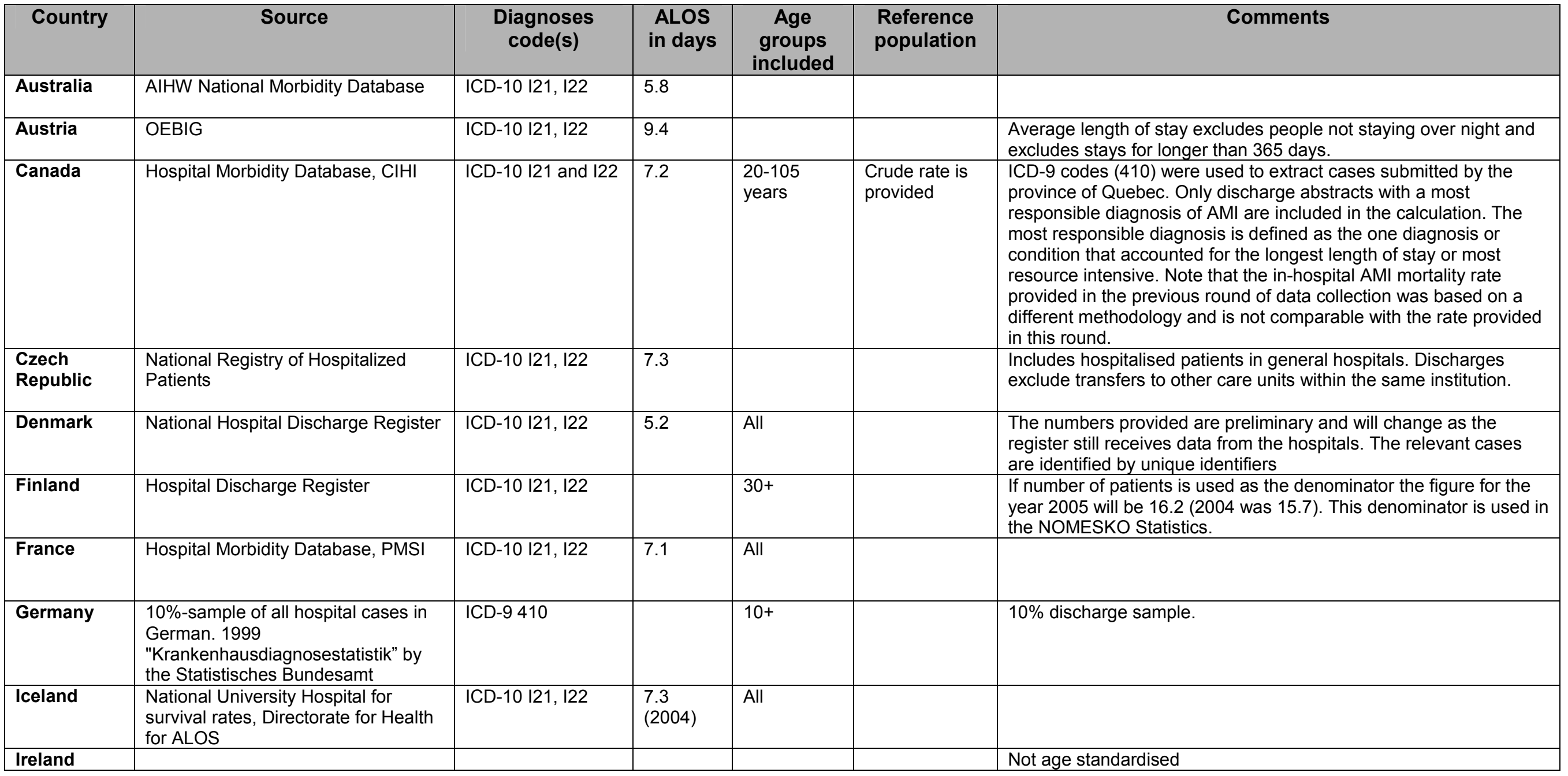


DELSA/HEA/WD/HWP(2007)4

\begin{tabular}{|c|c|c|c|c|c|c|}
\hline Country & Source & $\begin{array}{l}\text { Diagnoses } \\
\text { code(s) }\end{array}$ & $\begin{array}{l}\text { ALOS } \\
\text { in days }\end{array}$ & $\begin{array}{l}\text { Age } \\
\text { groups } \\
\text { included }\end{array}$ & $\begin{array}{l}\text { Reference } \\
\text { population }\end{array}$ & Comments \\
\hline Italy & Ministry of Health & ICD-9 410 & 8.43 & All & & \\
\hline Japan & $\begin{array}{l}\text { Patient Survey } 2005 \text {. Specially tallied } \\
\text { by the Ministry of Health, Labour and } \\
\text { Welfare }\end{array}$ & & & & & \\
\hline Korea & $\begin{array}{l}\text { Research on National Surveillance } \\
\text { for Cardiovascular disease }\end{array}$ & $\begin{array}{l}\text { I21, I22, I23, I250, } \\
\mathrm{I} 251\end{array}$ & 9.2 days & All & & $\begin{array}{l}\text { 1. Case definition: cases with } I 21, I 22, I 23, I 250 \text { and } I 251 \text { were } \\
\text { selected from the } 2004 \text { National Health Insurance Data. Medical } \\
\text { records have been reviewed to identify cases compatible with AHA- } \\
\text { EPI or ESC/ACC criteria. } 2 \text {. We calculated AMI } 28 \text { day in -Hospital } \\
\text { case fatality rate. }\end{array}$ \\
\hline Mexico & $\begin{array}{l}\text { Sistema Automatizado de Egresos } \\
\text { Hospitalarios, Dirección General de } \\
\text { Información en Salud, Secretaría de } \\
\text { Salud }\end{array}$ & ICD-10 I21, I22. & 6.5 & & & $\begin{array}{l}\text { Indicator coverage: discharges recorded in all hospitals providing } \\
\text { care to the uninsured population that is the population without } \\
\text { access to social security (approximately } 50 \% \text { of the total Mexican } \\
\text { population). The complete } 2005 \text { database analysed comprises } \\
1980962 \text { discharges reported by } 596 \text { hospitals run by the State } \\
\text { Health Services, } 10 \text { National Institutes of Health (Federal Tertiary } \\
\text { Care Hospitals) and } 6 \text { federal hospitals located in Mexico City and } \\
\text { run by the federal government. Federal psychiatric hospitals are } \\
\text { excluded from this database. It is estimated that this hospital } \\
\text { activity represents } 41 \% \text { of the total activity recorded by public } \\
\text { hospital facilities comprised in the National Health System (figure } \\
\text { estimated on the basis of } 2004 \text { data). It is expected that the } \\
\text { corresponding } 2005 \text { percentage will be slightly higher. }\end{array}$ \\
\hline Netherlands & $\begin{array}{l}\text { National Medical Registry, owned by } \\
\text { PRISMANT. Calculations and } \\
\text { methods by Statistics Netherlands in } \\
\text { cooperation with Institute for Public } \\
\text { Health and the Environment }\end{array}$ & ICD-9 410 & 7.56 & All & & $\begin{array}{l}\text { Based on admissions, although figures based on patients are } \\
\text { available. Rate defines: in-hospital mortality rate within } 30 \text { days of } \\
\text { admission, among all discharges with AMl as reason for admission. } \\
\text { Includes clinical admissions (with one overnight stay or more) and } \\
\text { admissions without an overnight stay (=day care). Discharges from } \\
\text { small specialised hospitals are excluded. }\end{array}$ \\
\hline $\begin{array}{l}\text { New } \\
\text { Zealand }\end{array}$ & $\begin{array}{l}\text { National Minimum Data Set (NMDS) } \\
2005 / 2006\end{array}$ & $\begin{array}{l}\text { ICD-10-AM } \\
\text { (Australian } \\
\text { Modification) I21- } \\
\text { I22. }\end{array}$ & 5.5 & All & & $\begin{array}{l}\text { Publicly funded events. If compared to unique patients, rather than } \\
\text { admissions, the rate is } 7.64 \% \text {. }\end{array}$ \\
\hline Norway & Norwegian Patientregister. & ICD-10 I21, I22 & & & & $\begin{array}{l}\text { Norwegian Patient register has no unique patient identifier. This } \\
\text { means that the same person may appear in the statistics several } \\
\text { times during the year if this person gets treatment in different } \\
\text { hospitals during the year. }\end{array}$ \\
\hline
\end{tabular}


DELSA/HEA/WD/HWP(2007)4

\begin{tabular}{|c|c|c|c|c|c|c|}
\hline Country & Source & $\begin{array}{l}\text { Diagnoses } \\
\text { code(s) }\end{array}$ & $\begin{array}{l}\text { ALOS } \\
\text { in days }\end{array}$ & $\begin{array}{l}\text { Age } \\
\text { groups } \\
\text { included }\end{array}$ & $\begin{array}{l}\text { Reference } \\
\text { population }\end{array}$ & Comments \\
\hline & Syndromes PL-ACS & $\begin{array}{l}\text { I22.Diagnosis } \\
\text { based on clinical } \\
\text { definitions of } \\
\text { STEMI and } \\
\text { NSTEMI }\end{array}$ & & & & $\begin{array}{l}\text { Acute Coronary Syndromes with diagnosis of STEMI or NSTEMI in } \\
\text { years 2004-2005. }\end{array}$ \\
\hline Portugal & $\begin{array}{l}\text { "Instituto de Gestão Informática e } \\
\text { Financeira da Saúde" - Health } \\
\text { Ministry }\end{array}$ & ICD-9 410 & & $15-105$ & & $\begin{array}{l}\text { This indicator was calculated with data from Diagnosis Related } \\
\text { Groups (DRGs) a national data base from "Instituto de Gestão } \\
\text { Informática e Financeira da Saúde" - Health Ministry. }\end{array}$ \\
\hline $\begin{array}{l}\text { Slovak } \\
\text { Republic }\end{array}$ & $\mathrm{NHIS}$ & ICD-10 I21, I22 & & & $\begin{array}{l}\text { Standard } \\
\text { European } \\
\text { population }\end{array}$ & \\
\hline Spain & $\begin{array}{l}\text { Hospital Discharge Minimum Data } \\
\text { Set (Conjunto Mínimo Básico de } \\
\text { Datos CMBD). Ministerio de Sanidad } \\
\text { y Consumo. Instituto de Información } \\
\text { Sanitaria. Registro de altas } 2004\end{array}$ & ICD-9 410 & 9.36 & All & & Public hospitals ( $75 \%$ discharges of total country). \\
\hline Sweden & $\begin{array}{l}\text { The Swedish Hospital Discharge } \\
\text { Register }\end{array}$ & ICD-10 I21, I22 & 5.3 & $0-85$ & & $\begin{array}{l}\text { The value for the standardised death rate was around } 0.08 \text { due to } \\
\text { two very high death rates for the youngest age groups with very few } \\
\text { AMl's. Crude (non standardised) death proportion almost the same: } \\
0.082630168 \text {. }\end{array}$ \\
\hline Switzerland & AMIS-plus & $\begin{array}{l}\text { ICD-10 I21, I22. } \\
\text { Acute myocardial } \\
\text { infarction: defined } \\
\text { by characteristic } \\
\text { symptoms and or } \\
\text { ECG changes and } \\
\text { enzyme rises (total } \\
\text { creatine kinase or } \\
\text { creatine kinase MB } \\
\text { fraction) at least } \\
\text { twice the upper } \\
\text { limit or normal }\end{array}$ & 7.7 & & & $\begin{array}{l}\text { Data show overall in-hospital mortality (not limited to } 30 \text { days); data } \\
\text { not based on a comprehensive national data base, but judged to be } \\
\text { representative. } \\
\text { The ICD-10 I21.4 code was used only as an example, to show that } \\
\text { the criteria of the AMIS-plus registry are not all exactly the same as } \\
\text { in ICD. In the AMIS-plus data registry are all cases with acute } \\
\text { coronary syndrome (but our expert extracted the cases for AMI). } \\
\text { The patients' data are entered in the data registry regardless if it's a } \\
\text { first infarction or a re-infarction. }\end{array}$ \\
\hline $\begin{array}{l}\text { United } \\
\text { Kingdom }\end{array}$ & $\begin{array}{l}\text { Hospital Episode Statistics (covers } \\
\text { all NHS trusts in England) }\end{array}$ & ICD-10 I21-I22 & & All & & $\begin{array}{l}\text { Based on in hospital mortality during the last finished consultant } \\
\text { episode in an inpatient spell. The data are for England only. The } \\
\text { age adjusted figure above is distorted by the fact that there was a } \\
\text { single admission in the 10-14 age group who died. }\end{array}$ \\
\hline
\end{tabular}


DELSA/HEA/WD/HWP(2007)4

\begin{tabular}{|l|l|l|l|l|l|l|}
\hline \multicolumn{1}{|c|}{ Country } & \multicolumn{1}{|c|}{ Source } & $\begin{array}{c}\text { Non-OECD EU countries } \\
\text { code(s) }\end{array}$ & $\begin{array}{c}\text { ALOS } \\
\text { in days }\end{array}$ & $\begin{array}{c}\text { Age } \\
\text { groups } \\
\text { included }\end{array}$ & $\begin{array}{c}\text { Reference } \\
\text { population }\end{array}$ & \multicolumn{1}{c|}{ Comments } \\
\hline Latvia & $\begin{array}{l}\text { Health Statistics and medical } \\
\text { technology state agency }\end{array}$ & ICD-10 I21, I22 & 11.7 & $18+$ & $\begin{array}{l}\text { Indicator - mortality of adolescents and adults (\%) in the hospital } \\
\text { from acute myocardial infarction. Numerator: deceased with } \\
\text { diagnosis acute myocardial infarction in the hospital. Denominator: } \\
\text { Number of hospitalised with diagnosis of acute myocardial } \\
\text { infarction. }\end{array}$ \\
\hline
\end{tabular}




\section{In-hospital mortality rate within 30 days of hospital admission for stroke}

\section{Operational Definition}

Numerator: Number of deaths in the hospital that occurred within 30 days of hospital admission with primary diagnosis of

a) hemorrhagic stroke (ICD-9 430-432 or ICD-10 I61-I62)

and b) ischemic stroke (ICD-9 433, 434, and 436 or ICD-10 I63-I64).

Denominator: Number of people hospitalised with primary diagnosis of stroke.

Table 14. In-hospital mortality rate within $\mathbf{3 0}$ days of hospital admission for stroke

\begin{tabular}{|c|c|c|c|c|c|}
\hline \multirow[b]{2}{*}{ Country } & \multirow[b]{2}{*}{ Data year } & \multicolumn{2}{|c|}{ Hemorrhagic mortality rate } & \multicolumn{2}{|c|}{ Ischemic mortality rate } \\
\hline & & $\begin{array}{c}\text { Hospital } \\
\text { admissions- } \\
\text { based }\end{array}$ & $\begin{array}{l}\text { Unique } \\
\text { identifiers }\end{array}$ & $\begin{array}{c}\text { Hospital } \\
\text { admissions- } \\
\text { based }\end{array}$ & $\begin{array}{c}\text { Unique } \\
\text { identifiers }\end{array}$ \\
\hline \multirow[t]{2}{*}{ Australia } & 2004-2005 & 24.9 & \multicolumn{3}{|c|}{11.9} \\
\hline & $2000-2001$ & 25.0 & \multicolumn{3}{|c|}{13.0} \\
\hline Austria & 2004 & 17.0 & \multicolumn{3}{|c|}{8.0} \\
\hline Canada & $2004-2005$ & 29.9 & \multicolumn{3}{|c|}{13.1} \\
\hline Czech Republic & 2004 & 28.4 & \multicolumn{3}{|c|}{12.0} \\
\hline \multirow[t]{2}{*}{ Denmark } & 2005 & \multicolumn{3}{|c|}{23.6} & 7.2 \\
\hline & 2004 & \multicolumn{3}{|c|}{25.4} & 7.0 \\
\hline \multirow[t]{3}{*}{ Finland } & 2005 & 12.5 & 22.9 & 6.3 & 10.5 \\
\hline & 2004 & 13.7 & 24.5 & \multirow[t]{2}{*}{6.5} & 10.6 \\
\hline & 2003 & & 24 & & 11 \\
\hline \multirow[t]{3}{*}{ France } & 2005 & 27.3 & \multicolumn{3}{|c|}{11.2} \\
\hline & 2003 & 27.5 & \multicolumn{3}{|c|}{13.5} \\
\hline & 2001 & 27.6 & \multicolumn{3}{|c|}{13.4} \\
\hline Germany $^{2}$ & 1999 & 21.0 & \multicolumn{3}{|c|}{10.9} \\
\hline \multirow[t]{3}{*}{ Iceland } & 2005 & 30.6 & \multicolumn{3}{|c|}{5.8} \\
\hline & 2004 & 39.2 & \multicolumn{3}{|c|}{6.3} \\
\hline & 2002 & 19.2 & \multicolumn{3}{|c|}{4.1} \\
\hline Ireland & 2003 & 23.9 & \multicolumn{3}{|c|}{11.3} \\
\hline \multirow[t]{3}{*}{ Italy $^{2}$} & 2004 & 24.3 & \multicolumn{3}{|c|}{8.5} \\
\hline & 2003 & 24.6 & \multicolumn{3}{|c|}{9.4} \\
\hline & 2001 & 29.5 & \multicolumn{3}{|c|}{12.2} \\
\hline \multirow[t]{2}{*}{ Japan } & 2005 & 10.9 & \multicolumn{3}{|c|}{3.3} \\
\hline & 1999 & 5.3 & \multicolumn{3}{|c|}{3.2} \\
\hline Korea $^{1}$ & 2004 & 35.0 & & 15.2 & \\
\hline Mexico & 2005 & 32.0 & & 20.1 & \\
\hline & 2004 & 29.3 & & 19.6 & \\
\hline
\end{tabular}


DELSA/HEA/WD/HWP(2007)4

\begin{tabular}{|c|c|c|c|c|c|}
\hline \multicolumn{6}{|c|}{ cont. } \\
\hline \multirow[b]{2}{*}{ Country } & \multirow[b]{2}{*}{ Data year } & \multicolumn{2}{|c|}{ Hemorrhagic mortality rate } & \multicolumn{2}{|c|}{ Ischemic mortality rate } \\
\hline & & $\begin{array}{c}\text { Hospital } \\
\text { admissions- } \\
\text { based }\end{array}$ & $\begin{array}{c}\text { Unique } \\
\text { identifiers }\end{array}$ & $\begin{array}{c}\text { Hospital } \\
\text { admissions- } \\
\text { based }\end{array}$ & $\begin{array}{l}\text { Unique } \\
\text { identifiers }\end{array}$ \\
\hline \multirow[t]{3}{*}{ Netherlands } & 2005 & 29.9 & 33.9 & 9.2 & 9.3 \\
\hline & 2004 & 30.5 & 33.8 & 10.6 & 10.8 \\
\hline & 2001 & 35.0 & & 16.0 & \\
\hline \multirow[t]{2}{*}{ New Zealand } & $2005-2006$ & & 30.9 & & 11.9 \\
\hline & $1999-2000$ & & 32.3 & & 13.9 \\
\hline \multirow[t]{2}{*}{ Norway } & 2005 & 19.0 & & 8.0 & \\
\hline & 2004 & 25.0 & & 9.0 & \\
\hline Poland & 2004-2005 & 36.9 & & 11.6 & \\
\hline \multirow[t]{2}{*}{ Portugal } & 2005 & 26.9 & & 9.4 & \\
\hline & 2004 & 25.0 & & 12.2 & \\
\hline \multirow[t]{2}{*}{ Slovak Republic $^{3}$} & 2005 & 28.5 & & 12.2 & \\
\hline & 2004 & 29.8 & & 12.7 & \\
\hline Spain & 2004 & 28.6 & & 11.5 & \\
\hline \multirow[t]{3}{*}{ Sweden } & 2005 & 18.6 & & 8.4 & \\
\hline & 2004 & 18.1 & & 9.2 & \\
\hline & 2001 & 24.3 & & 10.6 & \\
\hline \multirow[t]{2}{*}{ United Kingdom $^{2}$} & 2003-2004 & 15.6 & & 5.5 & \\
\hline & $2002-2003$ & 16.5 & & 9.9 & \\
\hline
\end{tabular}

Notes:

NA stands for "non available".

1. Data also include patients that died within 30 days after admission, out of hospital.

2. Data with a limited generalisability to national level. See sources and methods for more information.

3. Age-standardised to Standard European Population. 
DELSA/HEA/WD/HWP(2007)4

In-hospital mortality rate within 30 days of hospital admission for stroke, sources and methods

\begin{tabular}{|c|c|c|c|c|c|c|c|}
\hline Country & Source & $\begin{array}{c}\text { Hemorrhagic Diagnostic } \\
\text { Code }\end{array}$ & $\begin{array}{l}\text { ALOS } \\
\text { in } \\
\text { days }\end{array}$ & Ischemic Diagnostic Code & $\begin{array}{l}\text { ALOS } \\
\text { in } \\
\text { days }\end{array}$ & $\begin{array}{l}\text { Ages in } \\
\text { years }\end{array}$ & Comments \\
\hline Australia & $\begin{array}{l}\text { AlHW National Hospital } \\
\text { Morbidity Database }\end{array}$ & ICD-10 I61-I62 & 11.0 & ICD-10 I63-I64 & 11.5 & & $\begin{array}{l}\text { How are missing cases treated, i.e. patients } \\
\text { who are diagnosed and entered into the } \\
\text { system, but are lost to follow-up? Dropped out. }\end{array}$ \\
\hline Austria & OEBIG & $\begin{array}{l}\text { ICD-10 I61-I62 } \\
\text { Hospital discharge data. } \\
\text { Denominator: all I61 and I62 } \\
\text { primary diagnosis - Average } \\
\text { length of stay excludes people } \\
\text { not staying over night and } \\
\text { excludes stays for longer than } \\
365 \text { days. }\end{array}$ & 20.39 & $\begin{array}{l}\text { ICD-10 I63-I64 } \\
\text { Hospital discharge data. } \\
\text { Denominator: all I63 und } 64 \\
\text { primary diagnosis. Average } \\
\text { length of stay excludes people } \\
\text { not staying over night and } \\
\text { excludes stays for longer than } \\
365 \text { days. }\end{array}$ & 18.63 & & $\begin{array}{l}\text { How are missing cases treated, i.e. patients } \\
\text { who are diagnosed and entered into the } \\
\text { system, but are lost to follow-up? Dropped out. }\end{array}$ \\
\hline Canada & $\begin{array}{l}\text { Hospital Morbidity } \\
\text { Database, } \mathrm{CIHI}\end{array}$ & $\begin{array}{l}\text { ICD-10 I61-I62 } \\
\text { ICD-9 codes (430-432) were } \\
\text { used to extract cases submitted } \\
\text { by the province of Quebec. } \\
\text { Only discharge abstracts with a } \\
\text { most responsible diagnosis of } \\
\text { stroke are included in the } \\
\text { calculation. Note that the in- } \\
\text { hospital mortality rate provided } \\
\text { in the previous round of data } \\
\text { collection were based on a } \\
\text { different methodology and are } \\
\text { not comparable with the rate } \\
\text { provided in this round. }\end{array}$ & 15.1 & $\begin{array}{l}\text { ICD-10 I63-I64. } \\
\text { ICD-9 codes (433-434 and } \\
436 \text { ) were used to extract } \\
\text { cases submitted by the } \\
\text { province of Quebec. These } \\
\text { data used the most } \\
\text { responsible diagnosis field } \\
\text { only. The most responsible } \\
\text { diagnosis is defined as the } \\
\text { one diagnosis or condition } \\
\text { that can be described as } \\
\text { being the most responsible for } \\
\text { the patient's stay in hospital. } \\
\text { In the event that multiple } \\
\text { diagnoses are listed, select } \\
\text { the most responsible } \\
\text { diagnosis from the condition } \\
\text { associated with the longest } \\
\text { length of stay or most } \\
\text { resource intense. Note that } \\
\text { the in-hospital mortality rate } \\
\text { provided in the previous round }\end{array}$ & 16.0 & $20-105$ & $\begin{array}{l}\text { Reference population: Crude rate is provided. } \\
\text { How are missing cases treated, i.e. patients } \\
\text { who are diagnosed and entered into the } \\
\text { system, but are lost to follow-up? Not } \\
\text { applicable: All patients were followed-up until } \\
\text { death, discharge or at the end of the } 30 \text { day } \\
\text { follow-up period if still in the hospital. Deaths } \\
\text { that occurred in a non-acute care setting, e.g., } \\
\text { rehabilitation specialty hospital, nursing home } \\
\text { etc, are not captured. }\end{array}$ \\
\hline
\end{tabular}


DELSA/HEA/WD/HWP(2007)4

\begin{tabular}{|c|c|c|c|c|c|c|c|}
\hline Country & Source & $\begin{array}{l}\text { Hemorrhagic Diagnostic } \\
\text { Code }\end{array}$ & $\begin{array}{l}\text { ALOS } \\
\text { in } \\
\text { days }\end{array}$ & Ischemic Diagnostic Code & $\begin{array}{l}\text { ALOS } \\
\text { in } \\
\text { days }\end{array}$ & $\begin{array}{l}\text { Ages in } \\
\text { years }\end{array}$ & Comments \\
\hline & & & & $\begin{array}{l}\text { of data collection were based } \\
\text { on a different methodology } \\
\text { and are not comparable with } \\
\text { the rate provided in this } \\
\text { round. }\end{array}$ & & & \\
\hline $\begin{array}{l}\text { Czech } \\
\text { Republic }\end{array}$ & $\begin{array}{l}\text { National Registry of } \\
\text { Hospitalized Patients }\end{array}$ & ICD-10 I61-I62 & 18.3 & ICD-10 I63-I64 & 19.1 & & $\begin{array}{l}\text { Includes hospitalised patients in general } \\
\text { hospitals. Discharges exclude transfers to } \\
\text { other care units within the same institution } \\
\text { How are missing cases treated, i.e. patients } \\
\text { who are diagnosed and entered into the } \\
\text { system, but are lost to follow-up? Usually no } \\
\text { missing cases in hospital records. }\end{array}$ \\
\hline Denmark & $\begin{array}{l}\text { National Hospital } \\
\text { Discharge Register }\end{array}$ & ICD-10 I61-I62 & 10.2 & ICD-10 I63-164 & 4.5 & All & $\begin{array}{l}\text { The numbers provided are preliminary and will } \\
\text { change as the register still receives data from } \\
\text { the hospitals. All relevant cases are identified } \\
\text { by unique identifiers } \\
\text { How are missing cases treated, i.e. patients } \\
\text { who are diagnosed and entered into the } \\
\text { system, but are lost to follow-up? Dropped out. }\end{array}$ \\
\hline Finland & $\begin{array}{l}\text { Hospital Discharge } \\
\text { Registry }\end{array}$ & ICD-10 I61-I62 & 31.85 & ICD-9 433-434 and 436 & 34.13 & $30+$ & $\begin{array}{l}\text { If number of patients is used as the } \\
\text { denominator the figure for } 2005 \text { will be } 22.9 \\
\text { (hemorrhagic) / } 10.5 \text { (ischemic) ( } 2004 \text { was } \\
25.0 \text { (hemorrhagic) / } 10.6 \text { (ischemic)). This } \\
\text { denominator is used in the NOMESKO } \\
\text { Statistics. } \\
\text { How are missing cases treated, i.e. patients } \\
\text { who are diagnosed and entered into the } \\
\text { system, but are lost to follow-up? Dropped out. }\end{array}$ \\
\hline France & $\begin{array}{l}\text { Hospital Morbidity } \\
\text { Database }\end{array}$ & ICD-10 I61, I62. & 14.2 & ICD-10 163, I64 & 13.1 & All & \\
\hline Germany & $\begin{array}{l}10 \% \text {-sample of all } \\
\text { hospital cases in German }\end{array}$ & & & & & & $10 \%$ discharge sample \\
\hline
\end{tabular}


DELSA/HEA/WD/HWP(2007)4

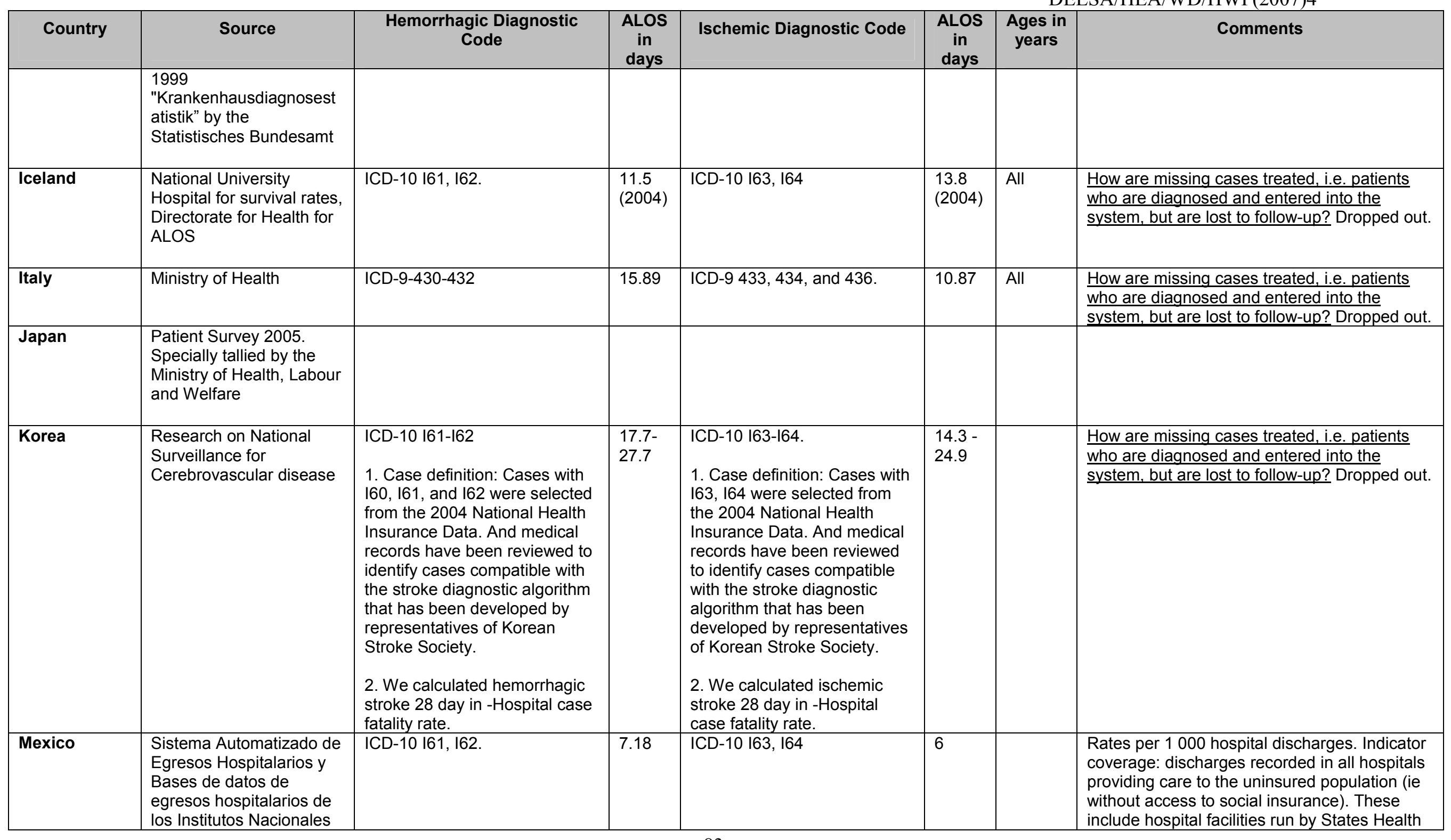


DELSA/HEA/WD/HWP(2007)4

\begin{tabular}{|c|c|c|c|c|c|c|c|}
\hline Country & Source & $\begin{array}{l}\text { Hemorrhagic Diagnostic } \\
\text { Code }\end{array}$ & $\begin{array}{l}\text { ALOS } \\
\text { in } \\
\text { days }\end{array}$ & Ischemic Diagnostic Code & $\begin{array}{l}\text { ALOS } \\
\text { in } \\
\text { days }\end{array}$ & $\begin{array}{c}\text { Ages in } \\
\text { years }\end{array}$ & Comments \\
\hline & $\begin{array}{l}\text { de Salud, Secretaría de } \\
\text { Salud }\end{array}$ & & & & & & $\begin{array}{l}\text { Services, National Institutes of Health and a } \\
\text { few hospitals run by the Federal government in } \\
\text { Mexico City. Therefore, hospital activity } \\
\text { undertaken at IMSS, ISSSTE, and other social } \\
\text { insurance schemes is not included in these } \\
\text { figures. Data reported in this table reflect about } \\
50 \% \text { of the total hospital activity undertaken by } \\
\text { public institutions in Mexico. } \\
\text { How are missing cases treated, i.e. patients } \\
\text { who are diagnosed and entered into the } \\
\text { system, but are lost to follow-up? Dropped out. }\end{array}$ \\
\hline Netherlands & $\begin{array}{l}\text { National Medical Registry, } \\
\text { owned by PRISMANT. } \\
\text { Calculations and methods } \\
\text { by Statistics Netherlands } \\
\text { in cooperation with } \\
\text { Institute for Public Health } \\
\text { and the Environment }\end{array}$ & ICD-9 430, 431 and 432 & 12.53 & ICD-9 433, 434 and 436 & 11.71 & All & $\begin{array}{l}\text { Based on admissions, although figures based } \\
\text { on patients are available. Rate defines: in- } \\
\text { hospital mortality rate within } 30 \text { days of } \\
\text { admission, among all discharges with AMI as } \\
\text { reason for admission. Includes clinical } \\
\text { admissions (with one overnight stay or more) } \\
\text { and admissions without an overnight stay } \\
\text { (=day care). Discharges from small specialised } \\
\text { hospitals are excluded. }\end{array}$ \\
\hline New Zealand & $\begin{array}{l}\text { National Minimum Data } \\
\text { Set (NMDS) 2005-2006 }\end{array}$ & $\begin{array}{l}\text { ICD-10-AM (Australian } \\
\text { Modification) I61-I62 }\end{array}$ & 6.72 & $\begin{array}{l}\text { ICD-10-AM (Australian } \\
\text { Modification) I63-I64 }\end{array}$ & 7.22 & All & $\begin{array}{l}\text { How are missing cases treated, i.e. patients } \\
\text { who are diagnosed and entered into the } \\
\text { system, but are lost to follow-up? All patients } \\
\text { were followed-up until death or } 30 \text { days from } \\
\text { admission if still in the hospital (including } \\
\text { rehabilitation). New Zealand has a unique } \\
\text { patient identifier and ability to follow-up } \\
\text { patients who suffered a stroke in any } \\
\text { setting until either their death or } 30 \text { days } \\
\text { after the admission. This includes } \\
\text { rehabilitation episodes and readmissions } \\
\text { irrespective of the reason. } \\
\text { Comments: Publicly funded events. }\end{array}$ \\
\hline Norway & Norwegian Patientregister & ICD-10 I61-162 & & ICD-10 I63-164 & & & $\begin{array}{l}\text { Norwegian Patientregister has no unique } \\
\text { patient identifier. This means that the same } \\
\text { person may appear in the statistics several }\end{array}$ \\
\hline
\end{tabular}


DELSA/HEA/WD/HWP(2007)4

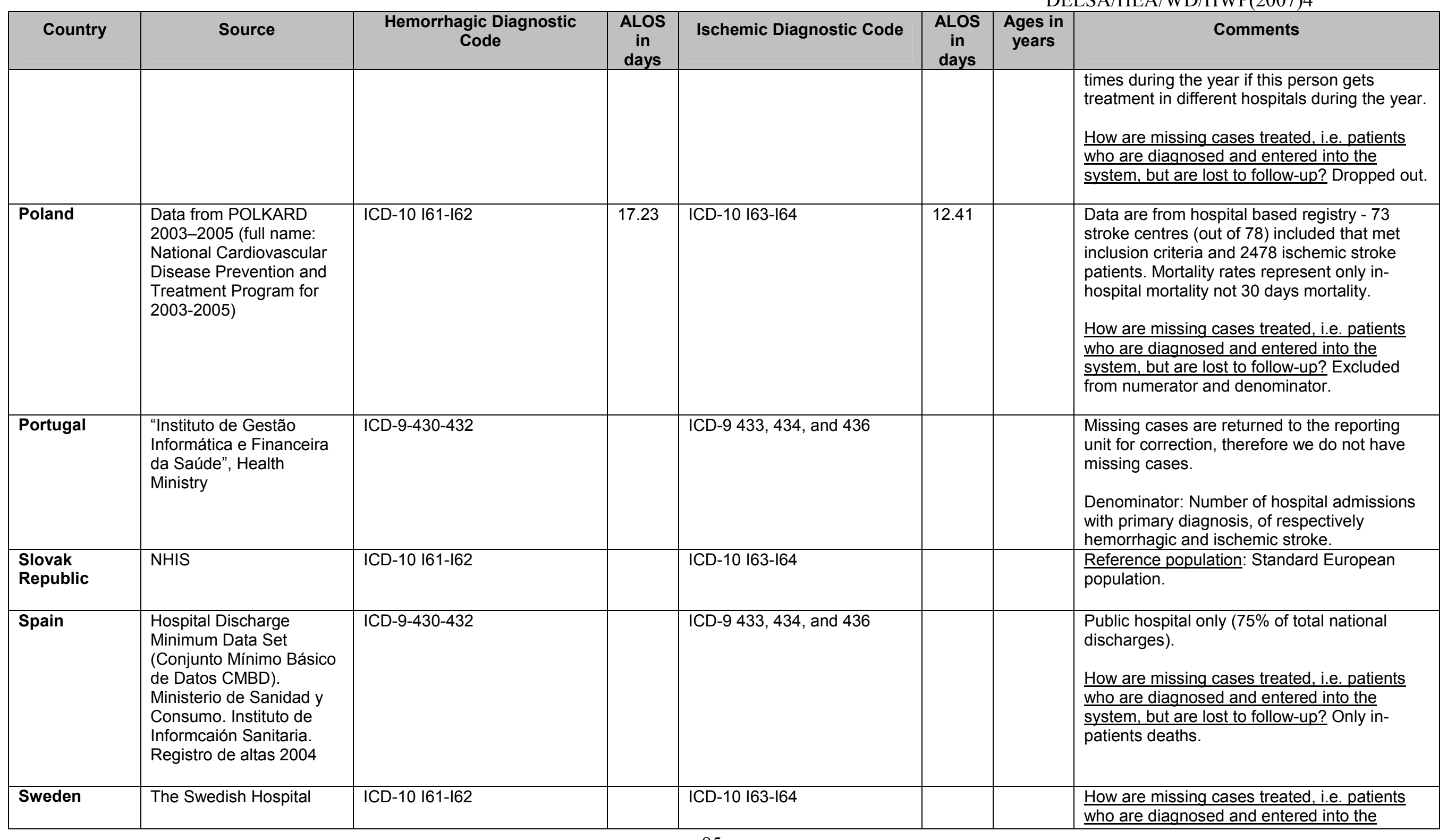


DELSA/HEA/WD/HWP(2007)4

\begin{tabular}{|c|c|c|c|c|c|c|c|}
\hline Country & Source & $\begin{array}{l}\text { Hemorrhagic Diagnostic } \\
\text { Code }\end{array}$ & $\begin{array}{l}\text { ALOS } \\
\text { in } \\
\text { days }\end{array}$ & Ischemic Diagnostic Code & $\begin{array}{l}\text { ALOS } \\
\text { in } \\
\text { days }\end{array}$ & $\begin{array}{l}\text { Ages in } \\
\text { years }\end{array}$ & Comments \\
\hline & Discharge Register & $\begin{array}{l}\text { The crude rate was } \\
0.186004677 \text { compared with } \\
0.078264327 \text { when } \\
\text { standardised to OECD } 1980 \\
\text { population which gives } \\
\text { difference due to strange } \\
\text { standard population that gives } \\
\text { enormous weights to strata } \\
\text { below } 45 \text { with very few strokes. } \\
\text { In Sweden no stroke patients } \\
\text { died at ages below } 35 \text {. }\end{array}$ & & $\begin{array}{l}\text { The crude rate was } \\
0.084118575 \text { compared to } \\
\text { only } 1.3 \% \text { when standardised } \\
\text { to OECD } 1980 \text { population. } \\
\text { Difference due to strange } \\
\text { standard population that gives } \\
\text { enormous weights to strata } \\
\text { below age } 45 \text { with very few } \\
\text { strokes. In Sweden no stroke } \\
\text { patients died at ages below } \\
35 \text {. }\end{array}$ & & & $\begin{array}{l}\text { system, but are lost to follow-up? Treated as } \\
\text { survivors. }\end{array}$ \\
\hline $\begin{array}{l}\text { United } \\
\text { Kingdom }\end{array}$ & $\begin{array}{l}\text { Hospital Episode } \\
\text { Statistics (covers all NHS } \\
\text { trusts in England) }\end{array}$ & ICD-10 I61-I62 & & ICD-10 I63-164 & & All & $\begin{array}{l}\text { Based on in-hospital mortality during the last } \\
\text { finished consultant episode in an in-patient } \\
\text { spell }\end{array}$ \\
\hline
\end{tabular}




\section{In-hospital waiting time for surgery after hip fracture, over age 65}

\section{Operational Definition}

Numerator: The number of patients with surgery initiated within 48 hours.

Denominator: The number of patients age 65 and older admitted to the hospital with a diagnosis of upper femur fracture (ICD-10 S72.0, S72.1, S72.2 or ICD-9 820).

Table 15. In-hospital waiting time for surgery after hip fracture, over age 65

\begin{tabular}{|c|c|c|}
\hline Country & $\begin{array}{c}\text { Femur fracture } \\
\text { operated within } 48 \\
\text { hours, age } 65+\text { per } 100\end{array}$ & Data year \\
\hline Austria & 80.0 & 2004 \\
\hline \multirow[t]{2}{*}{ Canada } & 77.8 & 2004-2005 \\
\hline & 79.5 & 2002 \\
\hline Czech Republic & 44.4 & 2004 \\
\hline \multirow[t]{2}{*}{ Denmark } & 57.6 & 2005 \\
\hline & 74.0 & 2004 \\
\hline \multirow[t]{4}{*}{ Finland } & 87.9 & 2005 \\
\hline & 86.1 & 2004 \\
\hline & 86.0 & 2003 \\
\hline & 87.7 & 2001 \\
\hline \multirow[t]{2}{*}{ Iceland $^{1}$} & 72.6 & $2002-2004$ \\
\hline & 73.1 & 1999-2003 \\
\hline \multirow[t]{3}{*}{ Italy } & 33.8 & 2004 \\
\hline & 32.7 & 2003 \\
\hline & 31.2 & 2001 \\
\hline \multirow[t]{2}{*}{ Mexico } & 50.0 & 2005 \\
\hline & 65.1 & 2003 \\
\hline \multirow[t]{2}{*}{ Netherlands } & 79.6 & 2004 \\
\hline & 80.4 & 2001 \\
\hline New Zealand & 72.3 & 2005-2006 \\
\hline \multirow[t]{3}{*}{ Norway } & 91.0 & 2005 \\
\hline & 93.0 & 2004 \\
\hline & 99.0 & 2003 \\
\hline \multirow[t]{3}{*}{ Portugal } & 47.4 & 2005 \\
\hline & 50.1 & 2004 \\
\hline & 32.1 & 2001 \\
\hline Spain & 32.9 & 2004 \\
\hline \multirow[t]{2}{*}{ Sweden } & 92.6 & 2004 \\
\hline & 93.5 & 2003 \\
\hline United Kingdom & 61.5 & 2002-2003 \\
\hline
\end{tabular}

Note:

1. Three-year average 
DELSA/HEA/WD/HWP(2007)4

In-hospital waiting time for surgery after hip fracture, over age 65, sources and methods

\begin{tabular}{|c|c|c|c|}
\hline Country & Source & Diagnosis Code(s) & Comments \\
\hline Austria & $\begin{array}{l}\text { Diagnoses and procedures } \\
\text { report }\end{array}$ & ICD-10 S72.0, S72.1, S72.2 & $\begin{array}{l}\text { In hospital waiting times in Austrian hospitals are not reported by the hour; but by the day. A waiting time of } \\
\text { two days can therefore in one case be longer than } 48 \text { hours, in another shorter. }\end{array}$ \\
\hline $\begin{array}{l}\text { Czech } \\
\text { Republic }\end{array}$ & $\begin{array}{l}\text { National Registry of } \\
\text { Hospitalized Patients }\end{array}$ & ICD-10 S72.0, S72.1, S72.2 & $\begin{array}{l}3 \text { days between date of arrival and date of surgery are used (based on dates not hours) included are } \\
\text { hospitalised patients in general hospitals. About half of patients with the diagnosis were not operated. }\end{array}$ \\
\hline Denmark & $\begin{array}{l}\text { National Hospital } \\
\text { Discharge Register }\end{array}$ & ICD-10 S72.0, S72.1, S72.2 & \\
\hline Finland & $\begin{array}{l}\text { Hospital Discharge } \\
\text { Registry }\end{array}$ & ICD-10 S72.0, S72.1, S72.2 & \\
\hline Iceland & $\begin{array}{l}\text { Directorate of Health in } \\
\text { Iceland }\end{array}$ & $\begin{array}{l}\text { ICD-10 S72.0, S72.1, S72.2 or } \\
\text { ICD-9 } 820 .\end{array}$ & $\begin{array}{l}\text { No missing cases. Indicator tracks surgery within two calendar days e.g. admission on January 1st, surgery } \\
\text { before January } 3 \text { rd. }\end{array}$ \\
\hline Italy & Ministry of Health & ICD-9 820 & \\
\hline Mexico & $\begin{array}{l}\text { Estadísticas Hospital de } \\
\text { Traumatología y Ortopedia } \\
\text { "Lomas Verdes" }\end{array}$ & ICD-10 S72.0, S72.1, S72.2 & $\begin{array}{l}\text { It represents } 7.23 \% \text { of total cases occurred in IMSS during } 2005 \text { and the } 50 \% \text { value it is for } 24 \text { hours and not } \\
\text { for } 48 \text { hour's period mentioned. On the hospital the intervals measured to initiate surgery go from } 0 \text { to } 24 \text { hrs, } \\
24 \text { to } 72 \text { hours and more than } 72 \text { hours to a week. The total cases obtained in this indicator was } 486 \text { it } \\
\text { represents } 7.23 \% \text { of the total cases during } 20056,719 \text {. The result could be extensible to all the institution }\end{array}$ \\
\hline Netherlands & $\begin{array}{l}\text { National Medical Registry, } \\
\text { owned by PRISMANT. } \\
\text { Calculations and methods } \\
\text { by Statistics Netherlands in } \\
\text { cooperation with Institute } \\
\text { for Public Health and the }\end{array}$ & ICD-9-CM code 820 & $\begin{array}{l}\text { The Dutch National Medical Registry does not have exact "time of admission" and "time of operation". } \\
\text { Consequently we use calendar days, and this indicator does not exactly measure "surgery initiated within } 48 \\
\text { hours" but overestimates this percentage. We are able to calculate this indicator based on } 1 \text {. Hospital } \\
\text { admissions, } 2 \text {. Each first admission of a patient within a one-year period, and } 3 \text {. First admission of a patient } \\
\text { not admitted in the } 5 \text { preceding years (thus, readmissions excluded). Also, reoperations can be excluded by } \\
\text { counting "patients" instead of "admissions". Figures are based on first admissions within a on-year period for }\end{array}$ \\
\hline
\end{tabular}




\begin{tabular}{|c|c|c|c|}
\hline \multirow[b]{2}{*}{ Country } & \multirow[b]{2}{*}{ Source } & \multirow{2}{*}{ Diagnosis Code(s) } & \multirow{2}{*}{ Comments } \\
\hline & & & \\
\hline & Environment & & $\begin{array}{l}\text { hip fracture, acute and elective surgery. Includes clinical admissions (with one overnight stay or more) and } \\
\text { admissions without an overnight stay (=day care). Discharges from small specialised hospitals are excluded. } \\
\text { We use a data file obtained by record linkage of the National Medical Registry to the Municipality Basis } \\
\text { Registry. Discharges were linked by date of birth, sex, date of admission and postal code. About } 85 \% \text { of all } \\
\text { discharges could be linked, so we could calculate rates on the basis of persons instead of discharges. } \\
\text { Because some groups do have a smaller probability of being linked, a correction is applied. As a } \\
\text { consequence, the calculated rates are representative for all patients discharged because of hip fracture in the } \\
\text { Netherlands. }\end{array}$ \\
\hline New Zealand & $\begin{array}{l}\text { National Minimum Data Set } \\
\text { (NMDS) 2005-200 }\end{array}$ & $\begin{array}{l}\text { ICD-10-AM (Australian } \\
\text { Modification) S72.0, S72.1, } \\
\text { S72.2 }\end{array}$ & $\begin{array}{l}\text { In-hospital hip fracture cases were excluded. The hour of the admission and surgery is not recorded in the } \\
\text { NMDS. This indicator refers to surgery within two calendar days of admission. The rate for the same or next } \\
\text { day surgery is } 55.6 \%\end{array}$ \\
\hline Norway & Norwegian Patientregister & ICD-10 S72.0, S72.1, S72.2 & Numerator: The number of patients with surgery initiated within 48 hours (ICD-10 S72.0, S72.1, S72.2) \\
\hline Portugal & $\begin{array}{l}\text { "Instituto de Gestão } \\
\text { Informática e Financeira da } \\
\text { Saúde", Health Ministry }\end{array}$ & ICD-9 820 & $\begin{array}{l}\text { This indicator was calculated with data from Diagnosis Related Groups (DRGs) a national data } \\
\text { base from "Instituto de Gestão Informática e Financeira da Saúde" - Health Ministry }\end{array}$ \\
\hline Spain & $\begin{array}{l}\text { Hospital Discharge } \\
\text { Minimum Data Set } \\
\text { (Conjunto Mínimo Básico } \\
\text { de Datos CMBD). } \\
\text { Ministerio de Sanidad y } \\
\text { Consumo. Instituto de } \\
\text { Información Sanitaria. } \\
\text { Registro de altas } 2004\end{array}$ & ICD-9 820 & $\begin{array}{l}\text { Calculated only for the cases with date of procedure ( } 15 \% \text { of total fractures). Public hospitals represent only } \\
\text { ( } 75 \% \text { of total national discharges). }\end{array}$ \\
\hline Sweden & $\begin{array}{l}\text { National Hip Fracture } \\
\text { Register }\end{array}$ & ICD-10 S72.0, S72.1, S72.2 & The register does not measure the exact time of arrival to hospital only the date \\
\hline $\begin{array}{l}\text { United } \\
\text { Kingdom }\end{array}$ & $\begin{array}{l}\text { Hospital Episode Statistics } \\
\text { (covers all NHS trusts in } \\
\text { England) }\end{array}$ & ICD-10 S72.0, S72.1 S72.2 & $\begin{array}{l}\text { Based on admission (epiorder }=1 \text { ) finished (epistat }=3 \text { ) consultant episodes. Numerator is calculated as the } \\
\text { number of primary operations (oper_ } 1 \text { ) carried out on date op_dte_ } 1 \text { within } 48 \text { hours of admission date } \\
\text { (admidate). We only include those who have either HRG chapter of H or primary operation OPCS-4 chapter of } \\
\text { W. The data are for England only }\end{array}$ \\
\hline
\end{tabular}


DELSA/HEA/WD/HWP(2007)4

Influenza vaccination, over age 65

Operational Definition

Numerator: Number offered an annual influenza vaccination.

Denominator: Number of adults over 65 years of age.

Table 16. Influenza vaccination, over age 65

\begin{tabular}{|l|c|c|}
\hline \multicolumn{1}{|c|}{ Country } & Annual percentage $\%$ & Data year \\
\hline Australia & 79.1 & 2004 \\
\hline Austria & 23.7 & 1999 \\
\hline Belgium & 59.6 & 2005 \\
\hline Canada & 66.5 & 2005 \\
\hline Czech Republic & 16.5 & 2002 \\
\hline Denmark & 55.3 & 2005 \\
\hline Finland & 52 & 2005 \\
\hline France & 68 & 2004 \\
\hline Germany & 63 & 2005 \\
\hline Hungary & 37.1 & 2005 \\
\hline Ireland & 63 & 2005 \\
\hline Italy & 68.3 & 2005 \\
\hline Japan & 48 & 2004 \\
\hline Korea & 77.2 & 2005 \\
\hline Mexico & 29.1 & 2003 \\
\hline Netherlands & 77 & 2005 \\
\hline New Zealand & 60.6 & 2005 \\
\hline Portugal & 41.6 & 2005 \\
\hline Slovak Republic & 22.9 & 2004 \\
\hline Spain & 70.1 & 2005 \\
\hline Switzerland & 54 & 2005 \\
\hline United Kingdom & 75 & 2005 \\
\hline United States & 64.6 & 2005 \\
\hline
\end{tabular}

Source: OECD Health Data 2007, July 07

Influenza vaccination, sources and methods

http://www.ecosante.org/OCDEENG/310030.html 


\section{Smoking rate}

\section{Operational Definition}

Numerator: Number of smokers.

Denominator: Total population.

Table 17. Smoking rate

\begin{tabular}{|l|c|c|}
\hline \multicolumn{1}{|c|}{ Country } & $\begin{array}{c}\text { Smoking Rate } \\
\text { \% }\end{array}$ & Year \\
\hline Australia & 17.7 & 2004 \\
\hline Austria & 36.3 & 1999 \\
\hline Belgium & 20.0 & 2005 \\
\hline Canada & 17.3 & 2005 \\
\hline Czech Republic & 24.3 & 2005 \\
\hline Denmark & 26.0 & 2004 \\
\hline Finland & 21.8 & 2005 \\
\hline France & 23.0 & 2004 \\
\hline Germany & 24.3 & 2003 \\
\hline Greece & 38.6 & 2004 \\
\hline Hungary & 30.4 & 2003 \\
\hline Iceland & 19.3 & 2006 \\
\hline Ireland & 27.0 & 2002 \\
\hline Italy & 23.0 & 2006 \\
\hline Japan & 26.3 & 2006 \\
\hline Korea & 25.3 & 2005 \\
\hline Mexico ${ }^{1}$ & 26.4 & 2002 \\
\hline Netherlands & 31.0 & 2005 \\
\hline New Zealand & 22.5 & 2005 \\
\hline Norway & 24.0 & 2006 \\
\hline Poland & 26.3 & 2004 \\
\hline Portugal & 17.0 & 2005 \\
\hline Slovak Republic & 24.3 & 2005 \\
\hline Spain & 28.1 & 2002 \\
\hline Sweden & 15.9 & 2005 \\
\hline Switzerland & 26.8 & 2.9 \\
\hline Turkey & 32.1 & 2005 \\
\hline United Kingdom & 24.0 & 2005 \\
\hline United States & 16.9 & 2005 \\
\hline
\end{tabular}

\begin{tabular}{|c|c|c|}
\hline \multicolumn{3}{|c|}{ Non-OECD EU countries } \\
\hline Country & $\begin{array}{c}\text { Smoking Rate } \\
\%\end{array}$ & Year \\
\hline Malta $^{2}$ & 23.4 & 2002 \\
\hline
\end{tabular}

Note:

1. Data refer to urban population only.

2. Data are specific to HCQI Project. Source: EUROSTAT:

http://epp.eurostat.ec.europa.eu/portal/

Source: OECD Health Data 2007, July 07

\section{Smoking rate, sources and methods}

http://www.ecosante.org/OCDEENG/813030.html 


\section{TWO NEW INDICATORS CONSIDERED FIT FOR INTERNATIONAL COMPARISONS: CLINICAL IMPORTANCE, SCIENTIFIC SOUNDNESS, SPECIFICATIONS AND DATA RESULTS}

46. This section presents information on two indicators that were reviewed by the HCQI Expert Group and found to be ready for publication in a working paper format. The decisions were guided by a review of the indicators according to criteria for scientific soundness, clinical and policy importance of the indicator and the comparability across countries of the indicator's data sources.

47. Since these indicators are being published for the first time, a full account is given here of their description and definition, scientific soundness, importance and data comparability issues related to the indicator. Also presented are the national estimates and the sources and methods for each indicator.

48. The following table presents a summary of the data availability for the two indicators presented in this section. 
Table 18. Summary of data availability for two new indicators in the 2006 HCQI Project indicator set

\begin{tabular}{|c|c|c|}
\hline $\begin{array}{l}\text { Country } \\
\text { /Indicator }\end{array}$ & Retinal Exams in Diabetics & Asthma Admission Rate \\
\hline \multicolumn{3}{|l|}{ Australia } \\
\hline \multicolumn{3}{|l|}{ Austria } \\
\hline \multicolumn{3}{|l|}{ Belgium } \\
\hline \multicolumn{3}{|l|}{ Canada } \\
\hline \multicolumn{3}{|l|}{ Czech Republic } \\
\hline \multicolumn{3}{|l|}{ Denmark } \\
\hline \multicolumn{3}{|l|}{ Finland } \\
\hline \multicolumn{3}{|l|}{ France } \\
\hline \multicolumn{3}{|l|}{ Germany } \\
\hline \multicolumn{3}{|l|}{ Greece } \\
\hline \multicolumn{3}{|l|}{ Hungary } \\
\hline \multicolumn{3}{|l|}{ Iceland } \\
\hline \multicolumn{3}{|l|}{ Ireland } \\
\hline \multicolumn{3}{|l|}{ Italy } \\
\hline \multicolumn{3}{|l|}{ Japan } \\
\hline \multicolumn{3}{|l|}{ Korea } \\
\hline \multicolumn{3}{|l|}{ Mexico } \\
\hline \multicolumn{3}{|l|}{ Netherlands } \\
\hline \multicolumn{3}{|l|}{ New Zealand } \\
\hline \multicolumn{3}{|l|}{ Norway } \\
\hline \multicolumn{3}{|l|}{ Poland } \\
\hline \multicolumn{3}{|l|}{ Portugal } \\
\hline \multicolumn{3}{|l|}{ Slovak Republic } \\
\hline \multicolumn{3}{|l|}{ Spain } \\
\hline \multicolumn{3}{|l|}{ Sweden } \\
\hline \multicolumn{3}{|l|}{ Switzerland } \\
\hline \multicolumn{3}{|l|}{ Turkey } \\
\hline \multicolumn{3}{|l|}{ United Kingdom } \\
\hline United States & & \\
\hline
\end{tabular}

\begin{tabular}{|l|l|l|}
\hline \multicolumn{2}{|c|}{ Non-OECD EU countries } \\
\multicolumn{1}{|c|}{$\begin{array}{c}\text { Country } \\
\text { IIndicator }\end{array}$} & Retinal Exams in Diabetics & Asthma Admission Rate \\
\hline Cyprus* & & \\
\hline Latvia & & \\
\hline Malta & & \\
\hline
\end{tabular}

(Blank/white cells indicate unavailability of data)

\footnotetext{
* See footnotes 2 and 3 on page 3 .
} 
DELSA/HEA/WD/HWP(2007)4

\section{Retinal exam in diabetics}

\section{Operational Definition}

Numerator: Number of diabetic patients who received a dilated eye exam or evaluation of retinal photography by an ophthalmologist or optometrist in a given year.

Denominator: Number of patients with diabetes (type I and type II) age 18-75 years.

49. Importance: Diabetes has become one of the most important public health challenges of the $21^{\text {st }}$ century. Over 150 million adults are affected worldwide with the number expected to double in the next 25 years (King et al., 1998; Zimmet et al., 2001). For example in the US an estimated 15.7 million people suffers diabetes, including an estimated 5.4 million people not yet diagnosed. The prevalence of diabetes in the US is projected to increase from the present rate of $5.9 \%$ to $8.9 \%$ by $2025 .{ }^{20}$ This rise is fuelled largely by the rise in obesity. The epidemic of diabetes requires resources to be devoted to the management of diabetes and its complications. Diabetes is the leading cause of blindness in industrialised countries in people ages 20-74 (Ghafour et al., 1983) and the most common cause of end-stage renal disease in the United States, Europe, and Japan. Individuals with type II diabetes have a 2-4 times greater risk of cardiovascular disease compared with people who do not have diabetes (Haffner, 2000). Nontraumatic amputations are 15 times more frequent in diabetic patients than in the general population (Ollendorf et al., 1998). While recent medical advances have led to a reduction in mortality from cardiovascular disease in OECD countries, such a positive trend has not been documented for diabetic patients, suggesting that these advances may be less effective for diabetics (Gu et al., 1999). Diabetes mellitus was responsible for an estimated 21 deaths per 100,000 people in WHO Euro A countries in 2000. This represents $2 \%$ of all deaths.

50. Retinopathy poses a serious threat to vision. In the United States, diabetes is responsible for $8 \%$ of legal blindness, making it the leading cause of new cases of blindness in adults 20-74 years of age. ${ }^{21}$ Each year, between 12,000 and 24,000 people lose their sight because of diabetes. Nearly all patients who have type I diabetes for about 20 years will have evidence of diabetic retinopathy. Up to $21 \%$ of people with type II diabetes have retinopathy when they are first diagnosed with diabetes, and most will eventually develop some degree of retinopathy.

51. Cost: In 2002, the cost of diabetes in the United States was an estimated USD 92 billion in medical expenditures and USD 40 billion in lost productivity (ADA, 2003). According to projections by the International Diabetes Federation, countries will be spending $7-13 \%$ of their healthcare budgets on diabetes care by the year 2025 (IDF, 2003).

\section{Scientific Soundness}

52. Face validity: The prevalence of retinopathy is strongly related to the duration and control of diabetes, rendering adequate glycemic control the key measure to prevent retinopathy. But even in patients with manifest retinopathy, treatment modalities exist that can delay progression and eventual blindness. ${ }^{22}$

\footnotetext{
${ }^{20}$ American Diabetes Association "Facts and Figures", http://www.diabetes.org; NIDDK "Diabetes Overview", http://www.niddk.nih.gov/health/diabetes/pubs/dmover/dmover.htm.

${ }^{21}$ American Diabetes Association, http://www.diabetes.org/diabetes-statistics/eye-complications.jsp. Accessed, 28/07/04.

22 American Diabetes Association: Clinical Practice Recommendations 2002. Diabetic Retinopathy (Position Statement).2002;25(sup.1):90-93. Available at: http://care.diabetesjournals.org/cgi/content/full/25/suppl_1/s90.
} 
People with proliferate retinopathy can reduce their risk of blindness by $95 \%$ with timely treatment and appropriate follow-up care. ${ }^{23}$ Because a person can be unaware of having retinopathy and not realise it, a regular check-up with an eye care professional is essential for early detection and treatment. Additionally, there have been several cost-effectiveness analyses of screening for diabetic retinopathy. Even though modelling techniques and component costs have differed substantially, the result of all the analyses is the same: screening for diabetic retinopathy saves vision at a relatively low cost, and the cost is less than the disability payments provided to people who would go blind in the absence of a screening programme. ${ }^{24}$

53. Construct validity: A number of associations, such as the American Association of Clinical Endocrinologists/American College of Endocrinology, American Diabetes Association, and American Academy of Ophthalmology, offer clinical guidelines recommending that annual eye exams be performed for patients with diabetes. In addition, annual retinal exams are one of five diabetes management tests recommended by the US Alliance on Diabetes Quality Improvement (which includes the American Diabetes Association). They recommend that for the patient group 29 years or younger that the first examination be made within 3-5 years after diagnosis of diabetes once the patient is age 10 or older, with a minimum routine of yearly follow-up. For the patient group 30 years an older, it is recommended that the first examination be conducted at the time of diagnosis of diabetes and with yearly minimum routine follow-ups. Women with diabetes who are planning to become pregnant should have a comprehensive eye examination and be counselled on the risk of developing retinopathy, and also have a comprehensive eye examination in the $1^{\text {st }}$ trimester with close follow up throughout pregnancy.

54. Reliability: Countries use national surveys to determine eye exam rates. These rates will be affected by national aspects of survey design such as the question used, sampling, and method of administering the survey. Survey questions are also sensitive to cultural differences in survey responses in different countries, potentially leading to recall bias.

\section{Feasibility}

55. Data availability: Retinal eye exam rates are available for ten countries (Table 19). Data are provided for the years 1999 to 2005. Some countries use slightly different age ranges. One country could only provide regional data. Another country provided data for retinal exams in the last two years. One country could only provide the rate of eye exams by diabetics. Countries obtained their data from population surveys, from patient records, or clinical surveys.

56. Comparability issues: The deviations in age and years are minor. Another minor problem is comparing a country using rate of "eye exams" in diabetics to the dilated eye exam. The different methods of collecting data represent major threats to comparability. There are two basic ways to obtain estimates for this indicator, population based surveys, and surveys at clinical sites or a review of patient records. Population based surveys rely on respondents to self-report their diabetes diagnosis and their most recent eye exam. Population based surveys are likely to capture diabetics who might not be regularly seeing a physician. However, there may be recall bias associated with these surveys - in that respondents may not accurately be able to remember their last exam. Data obtained from patient records are likely to be more accurate with respect to the frequency of tests, but exclude diabetics who do not seek regular medical care. For these reasons rates obtained from population based surveys should be compared with caution to rates obtained from clinical surveys or clinical records.

\footnotetext{
${ }^{23}$ National Eye Institute, http://www.nei.nih.gov/health/diabetic/retinopathy.asp\#15. Accessed, 28/07/04.

24 American Diabetes Association: Clinical Practice Recommendations 2002. Diabetic Retinopathy (Position Statement).2002; 25(sup.1):90-93. Available at: http://care.diabetesjournals.org/cgi/content/full/25/suppl_1/s90.
} 
57. Overall assessment: Only ten countries were able to provide data for this indicator. Not many countries routinely survey diabetics, or include such detailed questions in general population surveys. Obtaining data from patient records can be burdensome.

Table 19. Retinal exam in diabetics

\begin{tabular}{|l|c|c|}
\hline \multicolumn{1}{|c|}{ Country } & $\begin{array}{c}\text { Rate for } \\
\text { diabetics per } \\
\mathbf{1 0 0}\end{array}$ & Data year \\
\hline Australia & 72.5 & $1999-2000$ \\
\hline Canada & 48.6 & 2005 \\
\hline France & 45.1 & 2002 \\
\hline & 43.0 & 2001 \\
\hline Germany & 49.0 & 1998 \\
\hline Italy $^{1}$ & 56.0 & 2003 \\
\hline Japan & 37.0 & 2005 \\
\hline & 59.0 & 2002 \\
\hline Korea & 38.1 & 2005 \\
\hline New Zealand & 65.5 & 2005 \\
\hline Slovak Republic & 47.0 & 2005 \\
\hline Sweden & 77.8 & 2005 \\
\hline & 82.6 & 2003 \\
\hline United Kingdom & 83.4 & $2004-2005$ \\
\hline United States & 67.6 & 2002 \\
\hline \multicolumn{2}{l}{} \\
\hline
\end{tabular}

\begin{tabular}{|l|c|c|}
\hline \multicolumn{3}{|c|}{ Non-OECD EU countries } \\
\hline \multicolumn{3}{|c|}{ Retinal exams in diabetics } \\
\hline Latvia & 54.3 & 2005 \\
\hline
\end{tabular}

Notes:

1. Italy's figure refers to diabetic patients attending specialised clinics (estimated $60 \%$ of total diabetic population). 
Retinal exam in diabetics, sources and methods

\begin{tabular}{|c|c|c|c|c|c|c|c|}
\hline Country & Source & $\begin{array}{l}\text { Age } \\
\text { groups } \\
\text { included }\end{array}$ & $\begin{array}{l}\text { Deviations } \\
\text { from the } \\
\text { definition of } \\
\text { the } \\
\text { denominator }\end{array}$ & $\begin{array}{l}\text { Deviations } \\
\text { from the } \\
\text { definition of } \\
\text { the numerator }\end{array}$ & $\begin{array}{l}\text { Diabetes Diagnosis } \\
\text { Criteria: All registered } \\
\text { patients of diabetes } \\
\text { (type } 1 \text { and type II) } \\
\text { from Diabetic Register }\end{array}$ & $\begin{array}{c}\text { Additional } \\
\text { years } \\
\text { available }\end{array}$ & Comments \\
\hline Australia & $\begin{array}{l}\text { Australian Diabetes, Obesity } \\
\text { and Lifestyle Study (AusDiab) }\end{array}$ & $25+$ & & & & & $\begin{array}{l}\text { Numerator includes those screened in the last } 2 \text { years, as } \\
\text { that is the Australian recommendation. Nationally } \\
\text { representative sample }\end{array}$ \\
\hline Canada & $\begin{array}{l}\text { Canadian Community Health } \\
\text { Survey }\end{array}$ & & $\begin{array}{l}\text { Diabetic status } \\
\text { was self- } \\
\text { reported }\end{array}$ & $\begin{array}{l}\text { Numerator } \\
\text { comprised of } \\
\text { self-reported } \\
\text { diabetics who } \\
\text { have ever had } \\
\text { a dilation eye } \\
\text { exam. For the } \\
2005 \text { CCHS } \\
\text { survey, } 68.2 \% \\
\text { of diabetics } \\
\text { reported ever } \\
\text { having eye } \\
\text { dilation. }\end{array}$ & & & $\begin{array}{l}\text { The Diabetes Care module is optional content and } \\
\text { therefore, the results only represent diabetes care practices } \\
\text { in the participating health regions. In the } 2005 \mathrm{CCHS} \text {, the } \\
\text { module was selected by all health regions in Newfoundland } \\
\text { and Labrador, Prince Edward Island, New Brunswick, } \\
\text { Ontario, and Manitoba. The ability to generalise these } \\
\text { results to other non-participating provinces is limited. } \\
\text { Please describe the sample on which the indicator is } \\
\text { based: Data are from the } 2005 \text { Canadian Community } \\
\text { Health survey, a telephone survey conducted by Statistics } \\
\text { Canada from January to December } 2005 \text {. Diabetes-related } \\
\text { questions are from the Diabetes module. The diabetes } \\
\text { module was optional content and was selected by all } \\
\text { regions in the provinces of Newfoundland and Labrador, } \\
\text { Prince Edward Island, New Brunswick, Ontario, and } \\
\text { Manitoba. Only respondents in health regions where the } \\
\text { module was selected were administered the diabetes care } \\
\text { questions. All responses were voluntary. }\end{array}$ \\
\hline France & $\begin{array}{l}\text { "Entred" (survey based upon a } \\
\text { national sample of diabetic } \\
\text { patients whose health } \\
\text { insurance is "Caisse nationale } \\
\text { des travailleurs salariés") }\end{array}$ & $18+$ & & & & & National, generalisable sample \\
\hline Germany & $\begin{array}{l}\text { German National Health } \\
\text { Interview and Examination } \\
\text { Survey 1998; Thefeld W. }\end{array}$ & $18-75$ & & & & & $\begin{array}{l}\text { The validity of a self-reported diagnosis of diabetes mellitus } \\
\text { is compromised by potential misclassification bias (Thefeld } \\
\text { W. Prevalence of diabetes mellitus among adults in }\end{array}$ \\
\hline
\end{tabular}


DELSA/HEA/WD/HWP(2007)4

\begin{tabular}{|c|c|c|c|c|c|c|c|}
\hline Country & Source & $\begin{array}{c}\text { Age } \\
\text { groups } \\
\text { included }\end{array}$ & $\begin{array}{l}\text { Deviations } \\
\text { from the } \\
\text { definition of } \\
\text { the } \\
\text { denominator }\end{array}$ & $\begin{array}{l}\text { Deviations } \\
\text { from the } \\
\text { definition of } \\
\text { the numerator }\end{array}$ & $\begin{array}{l}\text { Diabetes Diagnosis } \\
\text { Criteria: All registered } \\
\text { patients of diabetes } \\
\text { (type } 1 \text { and type II) } \\
\text { from Diabetic Register }\end{array}$ & $\begin{array}{l}\text { Additional } \\
\text { years } \\
\text { available }\end{array}$ & Comments \\
\hline & $\begin{array}{l}\text { Prevalence of diabetes mellitus } \\
\text { among adults in Germany. } \\
\text { Gesundheitswesen 1999; S85- } \\
\text { S8 - the data presented here } \\
\text { are recalculated to the age } \\
\text { range } 18-75 \text { years. }\end{array}$ & & & & & & $\begin{array}{l}\text { Germany. Gesundheitswesen 1999; S85-S89). Detailed } \\
\text { information on research on this topic has been provided to } \\
\text { the OECD by German representatives and is available } \\
\text { upon request. Self-reported diagnosis by a physician. } \\
\text { Nationally representative sample }\end{array}$ \\
\hline Italy & $\begin{array}{l}\text { QuED study, Quality of Care } \\
\text { and Outcomes in Type } 2 \text { DB }\end{array}$ & All & & & & & $\begin{array}{l}\text { Comments: Based on sample of } 25274 \text { diabetic persons } \\
\text { attended in primary care-- data base of medical records. } \\
\text { National, generalisable sample }\end{array}$ \\
\hline Japan & Japan Medical Data Center & & & & & & \\
\hline Korea & $\begin{array}{l}\text { Korea National Health and } \\
\text { Nutrition Examination Survey }\end{array}$ & $30-39$ & & & & & $\begin{array}{l}\text { National representativity of the registry: National } \\
\text { representative sample. } 1 \text {. Diabetes diagnostic criteria: } \\
\text { Persons who answered "yes" to the questions: Have you } \\
\text { ever been told by doctor that you have diabetes? } 2 \text {. Age: } \\
\text { 19+3. Age group specific rate: } 19 \sim 29: 37.13,30 \sim 39: 17.56 \text {, } \\
40 \sim 49: 37.80,50 \sim 59: 38.20,60 \sim 69: 39.90,70+: 39.75\end{array}$ \\
\hline $\begin{array}{l}\text { New } \\
\text { Zealand }\end{array}$ & $\begin{array}{l}\text { National Get Checked Quality } \\
\text { Program for diabetics }\end{array}$ & & $\begin{array}{l}\text { Approximately } \\
70000 \text { diabetic } \\
\text { patients were } \\
\text { seen in the year } \\
2005 \text { with Get } \\
\text { Checked data } \\
\text { reported. }\end{array}$ & $\begin{array}{l}\text { Yes, diabetics } \\
\text { in New } \\
\text { Zealand are } \\
\text { screened } \\
\text { every second } \\
\text { year, hence } \\
\text { the rate differs } \\
\text { to the } \\
\text { retinopathy } \\
\text { screening } \\
\text { within } \\
\text { preceding } 2 \\
\text { years. } \\
\end{array}$ & & & \\
\hline $\begin{array}{l}\text { Slovak } \\
\text { Republic }\end{array}$ & Institute of health statistics & & & & & & $\begin{array}{l}\text { Data is not identical to request. Instead of number of retinal } \\
\text { exams is used number of retinopathy detected }\end{array}$ \\
\hline
\end{tabular}


DELSA/HEA/WD/HWP(2007)4

\begin{tabular}{|c|c|c|c|c|c|c|c|}
\hline Country & Source & $\begin{array}{l}\text { Age } \\
\text { groups } \\
\text { included }\end{array}$ & $\begin{array}{l}\text { Deviations } \\
\text { from the } \\
\text { definition of } \\
\text { the } \\
\text { denominator }\end{array}$ & $\begin{array}{l}\text { Deviations } \\
\text { from the } \\
\text { definition of } \\
\text { the numerator }\end{array}$ & $\begin{array}{l}\text { Diabetes Diagnosis } \\
\text { Criteria: All registered } \\
\text { patients of diabetes } \\
\text { (type } 1 \text { and type II) } \\
\text { from Diabetic Register }\end{array}$ & $\begin{array}{l}\text { Additional } \\
\text { years } \\
\text { available }\end{array}$ & Comments \\
\hline Sweden & NDR Sweden & & & & & & \\
\hline $\begin{array}{l}\text { United } \\
\text { Kingdom }\end{array}$ & $\begin{array}{l}\text { Quality and Outcomes } \\
\text { Framework (QOF), Health and } \\
\text { Social Care Information Centre }\end{array}$ & $16+$ & & & & & \\
\hline $\begin{array}{l}\text { United } \\
\text { States }\end{array}$ & $\begin{array}{l}\text { Agency for Healthcare } \\
\text { Research and Quality, Center } \\
\text { for Financing and Cost Trends, } \\
\text { Medical Expenditure Panel } \\
\text { Survey }\end{array}$ & $18+$ & & & & & $\begin{array}{l}\text { Persons answering "yes" to the following question: Have } \\
\text { you ever been told by a doctor or other health professional } \\
\text { that you have diabetes or sugar diabetes? Nationally } \\
\text { representative sample }\end{array}$ \\
\hline
\end{tabular}

\begin{tabular}{|c|c|c|c|c|c|c|c|}
\hline \multicolumn{8}{|c|}{ Non-OECD EU countries } \\
\hline Country & Source & $\begin{array}{l}\text { Age } \\
\text { groups } \\
\text { included }\end{array}$ & $\begin{array}{l}\text { Deviations } \\
\text { from the } \\
\text { definition of } \\
\text { the } \\
\text { denominator }\end{array}$ & $\begin{array}{l}\text { Deviations } \\
\text { from the } \\
\text { definition of } \\
\text { the numerator }\end{array}$ & $\begin{array}{l}\text { Diabetes Diagnosis } \\
\text { Criteria: All registered } \\
\text { patients of diabetes } \\
\text { (type } 1 \text { and type II) } \\
\text { from Diabetic Register }\end{array}$ & $\begin{array}{l}\text { Additional } \\
\text { years } \\
\text { available }\end{array}$ & Comments \\
\hline Latvia & $\begin{array}{l}\text { Register of the Patients of } \\
\text { Diabetes Mellitus }\end{array}$ & & & & $\begin{array}{l}\text { All registered patients of } \\
\text { diabetes (type } 1 \text { and } \\
\text { type II) from Diabetic } \\
\text { Register. }\end{array}$ & & $\begin{array}{l}\text { Diabetic Register does not cover all population of diabetics, } \\
\text { because there are unregistered cases yet. It includes the } \\
\text { patients who are in primary care and those in hospitals who } \\
\text { are registered by their physician as a diabetic in the } \\
\text { register. }\end{array}$ \\
\hline
\end{tabular}


DELSA/HEA/WD/HWP(2007)4

\section{Asthma admission rate}

\section{Operational Definition}

Source: AHRQ Prevention Quality Indicators.

Numerator: All non-maternal discharges of age 18 years and older with ICD-9-CM principal diagnosis code of asthma.

\section{Denominator: Total population.}

58. Importance: Asthma is the most common chronic disease in childhood, with increasing prevalence in recent decades. Research suggests that asthma may in fact be a collection of different diseases with similar symptoms (Wenzel, 2006). Asthma is an inherently treatable disease through appropriate medical care. In 2002, the estimated annual cost of treating asthma in the United States was USD 14 billion, of which hospital care accounted for almost a third of direct costs (NHLBI, 2002). Estimates for the European Union suggest annual medical costs of EUR 17.7 billion and productivity losses of EUR 9.8 billion (ERS, 2003). The cost of asthma treatments accounts for close to $2 \%$ of annual expenditures for medical care in Japan (Tanihara and Kobayashi, 2004). Analysis from Korea has shown that hospitalisations for asthma were a significant health care cost however hospitalisations and trips to the emergency department were only a small fraction of the total health care cost of uncontrolled asthma (Park, 2006).

59. Clinical significance of process or outcome: Avoidable hospitalisations are those conditions that could have been avoided if proper ambulatory care had been received and can thus be seen as a measure of access to appropriate medical care. While not all admissions for ambulatory care sensitive conditions are avoidable, appropriate prior ambulatory care can prevent most cases of the onset of this type of illness or condition, control an acute episodic illness or condition, or manage a chronic disease or condition. Hence a disproportionately high rate is presumed to reflect problems in obtaining access to primary care (Weisman, 1992). Admission rates for asthma have been shown to be associated with lower socioeconomic status (AHRQ, 2006).

60. Identification of process/outcome as quality problem: The rate of Ambulatory Care Sensitive (ACS) hospitalisations is considered an index of access of a population to adequate primary care. Primary care should be able to effectively manage both adults and children with asthma. Treatment with anti-inflammatory agents, such as inhaled corticosteroids and leukotriene inhibitors, are largely able to prevent exacerbation and, when it occurs, systemic corticosteroids and bronchodilators should preclude any need for hospitalisation. While current protocols and guidelines provide clear guidance for the treatment of asthma, studies suggests that treatment often falls short of recommended care (Mattke et al., 2006b; Halterman, 2001; and AAFA, 2005). As a consequence of insufficient treatment, patients with asthma may need to be hospitalised. Admission rates for asthma and asthma mortality rates have been used to assess quality of care. For example, the UK National Health Service has designated asthma admission as a High Level Performance Indicator, and both paediatric and adult admission rates are part of the US National Healthcare Quality Report (AHRQ, 2006). Asthma mortality rates have been used as an indicator to assess the quality of care for health system comparison in the European Community, United Kingdom, Australia, and several other countries (Charlton et al., 1998; Holland et al., 1997; Manuel and Mao, 2002; AIHW; 2003).

61. Policy importance: Given the high cost of hospital care and the high prevalence of asthma, elevated ACS hospitalisation rates could point not only towards possibilities to improve quality but also to substantial cost savings, if better primary care were provided. In addition, the ACS hospitalisation rate appears sensitive to the presence or absence of economic barriers to access. It has been reported to be lower and/or less correlated with socioeconomic status in countries with national health insurance (Billings, 1996).

62. Susceptibility to being influenced by the health system: Appropriate prior ambulatory care could prevent the onset of an illness or condition; control an acute episodic illness or condition; or manage a chronic disease or condition (Anderson, 1996). According to the National Asthma Education Program, asthma is a 
readily treatable chronic disease that can be managed effectively in the outpatient setting (NHLBI, 1997). In a US-based study of asthma in urban settings, low cost primary care and preventive measures significantly decreased the incidence of severe asthma episodes and hospitalisations (Houck, 2006). Observational studies offer some evidence that inhaled steroids may decrease risk of admission by up to $50 \%$ (Blaise, 1998).

\section{Scientific Soundness}

63. Face validity: Managing chronic diseases to prevent complications and exacerbations is regarded as a core task of the primary health care system. Little empirical evidence exists as to the validity of the asthma avoidable hospitalisations indicator in particular. The AHRQ study group developing this indicator found that the indicator was "adequately precise" in measuring true differences across areas or regions (AHRQ, 2006). However, there are important differences across race and socioeconomic groups and proper adjustment techniques when looking at sub national data is recommended (Ray, 1998).

64. Content validity: As mentioned above, several groups have advocated measures of ACS hospitalisation rates. The fact that hospital admission diagnoses are readily available in most countries implies that the indicator can be easily constructed. However, it should be mentioned that there remains some controversy about this (and similar) measures as a quality indicator, because ACS hospitalisation rates reflect access to, as well as quality of, primary care. Also, defining the appropriate level of hospital admission rates for those conditions is difficult, because in a subset of cases an admission is clearly warranted.

Table 20. Asthma admission rate per 10000 discharges, (primary care and prevention, ambulatory sensitive conditions)

\begin{tabular}{|l|c|c|}
\hline \multicolumn{1}{|c|}{ Country } & $\begin{array}{c}\text { Rate per } \\
\mathbf{1 0 0 0 0}\end{array}$ & Data year \\
\hline Australia & 10.05 & $2004-2005$ \\
\hline Austria & 6.97 & 2004 \\
\hline Canada & 3.73 & $2004-2005$ \\
\hline Czech Republic & 6.30 & 2004 \\
\hline Denmark & 5.64 & 2005 \\
\hline Finland & 13.23 & 2005 \\
\hline Iceland & 1.69 & 2005 \\
\hline Italy & 3.82 & 2004 \\
\hline Japan & 6.72 & 2005 \\
\hline Mexico & 1.82 & 2005 \\
\hline Netherlands & 2.78 & 2005 \\
\hline New Zealand & 8.13 & $2005-2006$ \\
\hline Norway & 4.54 & 2005 \\
\hline Portugal & 3.88 & 2004 \\
\hline Spain & 4.40 & 2004 \\
\hline Sweden & 3.30 & 2004 \\
\hline United States & 12.00 & 2002 \\
\hline
\end{tabular}

\begin{tabular}{|c|c|c|}
\hline \multicolumn{3}{|c|}{ Non-OECD EU countries } \\
\hline Country & $\begin{array}{c}\text { Rate per } \\
10000\end{array}$ & Data year \\
\hline Latvia $^{\top}$ & 16.13 & 2005 \\
\hline
\end{tabular}

Notes:

1. Data from hospital administered statistics 
DELSA/HEA/WD/HWP(2007)4

Asthma admission rate, sources and methods

\begin{tabular}{|c|c|c|c|c|c|c|c|}
\hline Country & Source & $\begin{array}{c}\text { Diagnosis } \\
\text { code(s) }\end{array}$ & $\begin{array}{l}\text { Age } \\
\text { groups } \\
\text { included }\end{array}$ & $\begin{array}{l}\text { Deviations } \\
\text { from the } \\
\text { definition of } \\
\text { the } \\
\text { denominator }\end{array}$ & $\begin{array}{l}\text { Deviations } \\
\text { from the } \\
\text { definition of } \\
\text { the numerator }\end{array}$ & $\begin{array}{l}\text { Additional } \\
\text { years } \\
\text { available }\end{array}$ & Comments \\
\hline Australia & $\begin{array}{l}\text { AlHW National Hospital } \\
\text { Morbidity Database }\end{array}$ & & & & $\begin{array}{l}\text { Data are for } \\
\text { ICD-10-AM } \\
\mathrm{J} 45, \mathrm{~J} 46\end{array}$ & & \\
\hline Austria & Statistics Austria & & $15+$ & & $\begin{array}{l}\text { Principle } \\
\text { diagnoses } \\
\text { given in ICD- } \\
10 \text { codes: } \\
\text { J450, J451, } \\
\text { J458, J459, } \\
\text { J46 }\end{array}$ & & $\begin{array}{l}\text { No exclusions were made, all discharges with principle } \\
\text { diagnosis of the given codes. }\end{array}$ \\
\hline Canada & $\begin{array}{l}\text { Discharge Abstract Database, } \\
\mathrm{CIHI}\end{array}$ & & & & & & $\begin{array}{l}\text { Cases were extracted based on a most responsible diagnosis } \\
\text { of the condition of interest. The most responsible diagnosis is } \\
\text { defined as the one diagnosis or condition that can be } \\
\text { described as being the most responsible for the patient's stay } \\
\text { in hospital. In the event that multiple diagnoses are listed, } \\
\text { select the most responsible diagnosis from the condition } \\
\text { associated with the longest length of stay or most resource } \\
\text { intense. }\end{array}$ \\
\hline $\begin{array}{l}\text { Czech } \\
\text { Republic }\end{array}$ & $\begin{array}{l}\text { National Registry of Hospitalized } \\
\text { Patients }\end{array}$ & & $20+$ & $\begin{array}{l}\text { Population } 20+ \\
\text { as of } 1 \text { st of } \\
\text { July }\end{array}$ & $\begin{array}{l}\text { Includes all } \\
\text { hospitalised } \\
\text { patients in } \\
\text { general } \\
\text { hospitals for } \\
\text { diagnoses of } \\
\text { J45, J46. }\end{array}$ & & \\
\hline Denmark & $\begin{array}{l}\text { National Hospital Discharge } \\
\text { Register }\end{array}$ & & & & & & $\begin{array}{l}\text { There have been no exclusions in the numerator (it is not } \\
\text { possible to write in the numerator box). The numbers provided } \\
\text { are preliminary and will change as the register still receives } \\
\text { data from the hospitals. It should be kept in mind, when }\end{array}$ \\
\hline
\end{tabular}


DELSA/HEA/WD/HWP(2007)4

\begin{tabular}{|c|c|c|c|c|c|c|c|}
\hline Country & Source & $\begin{array}{l}\text { Diagnosis } \\
\text { code(s) }\end{array}$ & $\begin{array}{l}\text { Age } \\
\text { groups } \\
\text { included }\end{array}$ & $\begin{array}{l}\text { Deviations } \\
\text { from the } \\
\text { definition of } \\
\text { the } \\
\text { denominator }\end{array}$ & $\begin{array}{l}\text { Deviations } \\
\text { from the } \\
\text { definition of } \\
\text { the numerator }\end{array}$ & $\begin{array}{l}\text { Additional } \\
\text { years } \\
\text { available }\end{array}$ & Comments \\
\hline & & & & & & & $\begin{array}{l}\text { interpreting this indicator that the indicator is strongly } \\
\text { dependent on how the health system is organised. }\end{array}$ \\
\hline Finland & Hospital Discharge Register & & & & & & $\begin{array}{l}\text { Overall number of cases was } 5893 . \text { In this all referred cases } \\
\text { are included. The number of cases admitted to hospital from } \\
\text { ER's was } 4214 \text {. Other cases were referrals. }\end{array}$ \\
\hline Italy & $\begin{array}{l}\text { Ministry of Health - National } \\
\text { discharges database }\end{array}$ & & & & & $2001+$ & \\
\hline Japan & Patient Survey & & $20+$ & & & & $\begin{array}{l}\text { Since the Patient Survey covers only one month, the } \\
\text { numerator (estimated } 7300 \text { ) was multiplied by } 12 \text {. The decline } \\
\text { of the rate (with respect to 2002) indicates improved quality of } \\
\text { care for asthma. Quoting the actual data provided by the } \\
\text { Patient Survey in } 2002 \text { and } 2005 \text {, the estimated number of } \\
\text { discharges over } 20 \text { years with primary Dx of asthma was } 7 \\
300 \text { in } 2002 \text { and } 5800 \text { in } 2005 \text {. The rate was calculated as } \\
\text { follows: } 5800 \text { X } 12 \text { months } / 103.56 \text { million population. }\end{array}$ \\
\hline Mexico & $\begin{array}{l}\text { SIAIS.- Sistema de Información } \\
\text { de Atención Integral a la Salud. } \\
\text { IMSS (DTIES) División Técnica } \\
\text { de Información Estadística en } \\
\text { Salud }\end{array}$ & & $20+$ & $\begin{array}{l}20 \text { years and } \\
\text { older. }\end{array}$ & $\begin{array}{l}20 \text { years and } \\
\text { older and ICD- } \\
10 \text { codes. } \\
\text { (Unofficial } \\
\text { table of ICD-9 } \\
\text { and ICD-10 } \\
\text { codes). }\end{array}$ & & Nationwide representative. \\
\hline Netherlands & $\begin{array}{l}\text { National Medical Registry, } \\
\text { owned by PRISMANT. } \\
\text { Calculations and methods by } \\
\text { Statistics Netherlands in } \\
\text { cooperation with Institute for } \\
\text { Public Health and the } \\
\text { Environment }\end{array}$ & & $18+$ & & & & $\begin{array}{l}\text { The Dutch National Medical Registry does not use the } \\
\text { following asthma diagnoses: } 493 . \times 2,493.2 x, 493.8 x,(493.81 \text {, } \\
\text { and } 493.82 \text { ). Consequently, our selection is based on ICD-9- } \\
\text { CM code } 493 \text { (493.0, } 493.1 \text { and } 493.9 \text { ). A fifth digit } \\
\text { differentiates between 'without mention of status asthmaticus' } \\
\text { and 'with status asthmaticus' (respectively codes } 0 \text { and } 1 \text { ). } \\
\text { Major Diagnostic Categories (MDCs) are not used in all } \\
\text { countries, at least not in the Netherlands. We assume that } \\
\text { MDC } 14 \text { refers to health status described in Chapter } 11 \text { of } \\
\text { ICD-9 (codes } 630-676 \text { ) and several V-codes (V22-V24, V27, } \\
\text { V28). Secondary diagnoses excluded from the numerator: }\end{array}$ \\
\hline
\end{tabular}


DELSA/HEA/WD/HWP(2007)4

\begin{tabular}{|c|c|c|c|c|c|c|c|}
\hline Country & Source & $\begin{array}{l}\text { Diagnosis } \\
\text { code(s) }\end{array}$ & $\begin{array}{c}\text { Age } \\
\text { groups } \\
\text { included }\end{array}$ & $\begin{array}{c}\text { Deviations } \\
\text { from the } \\
\text { definition of } \\
\text { the } \\
\text { denominator }\end{array}$ & $\begin{array}{l}\text { Deviations } \\
\text { from the } \\
\text { definition of } \\
\text { the numerator }\end{array}$ & $\begin{array}{l}\text { Additional } \\
\text { years } \\
\text { available }\end{array}$ & Comments \\
\hline & & & & & & & $\begin{array}{l}277.0,747.21,748.3,748.4,748.5,748.6,748.8,748.9, \\
750.3,759.3,770.7, \text { ICD-9 codes: } 630-676 \text { or V22-V24, V27, } \\
\text { V28. Discharges from small specialised hospitals are } \\
\text { excluded. We only present data from general acute care } \\
\text { hospitals (including university hospitals). Data from seven } \\
\text { specialised clinics for rehabilitation of chronic diseases like } \\
\text { lung disease were excluded. In these clinics patients are } \\
\text { admitted with the goal to learn to manage their disease. The } \\
\text { length of stay can be one day (day care) to several weeks. In } \\
\text { general, patients are not admitted in these clinics because of } \\
\text { acute exacerbations or complications. In all rates, clinical } \\
\text { admissions (with one overnight stay or more) and admissions } \\
\text { without an overnight stay (=day care) were included. } \\
\text { Transfers are included in the nominator. Consequently, } \\
\text { discharges from secondary hospitals are included. }\end{array}$ \\
\hline $\begin{array}{l}\text { New } \\
\text { Zealand }\end{array}$ & $\begin{array}{l}\text { National Minimum Data Set } \\
\text { (NMDS) 2005-2006. }\end{array}$ & $\begin{array}{l}\text { ICD-10- } \\
\text { AM } \\
\text { (Australian } \\
\text { Modificatio } \\
\text { n) J45-J46 }\end{array}$ & & & $\begin{array}{l}\text { Yes, excludes } \\
\text { pregnancy, } \\
\text { childbirth, } \\
\text { puerperium } \\
\text { (MDC14) and } \\
\text { emergency } \\
\text { specialty } \\
\text { (M05-08) with } \\
\text { a length of } \\
\text { stay < } 1 \text { day. }\end{array}$ & & Publicly funded events. \\
\hline Norway & Norwegian Patientregister. & & & & & & Diagnoses codes ICD-10: J45, J46 \\
\hline Portugal & DRGs & & & & & & \\
\hline $\begin{array}{l}\text { Slovak } \\
\text { Republic }\end{array}$ & & & & & & & $\begin{array}{l}\text { Our records do not work with four digits ICD; therefore it is not } \\
\text { possible to consider exclusions. }\end{array}$ \\
\hline Spain & $\begin{array}{l}\text { Hospital Discharge Minimum } \\
\text { Data Set (Conjunto Mínimo } \\
\text { Básico de Datos CMBD). }\end{array}$ & & & & & & only public hospitals ( $75 \%$ of total country discharges) \\
\hline
\end{tabular}


DELSA/HEA/WD/HWP(2007)4

\begin{tabular}{|c|c|c|c|c|c|c|c|}
\hline Country & Source & $\begin{array}{l}\text { Diagnosis } \\
\text { code(s) }\end{array}$ & $\begin{array}{c}\text { Age } \\
\text { groups } \\
\text { included }\end{array}$ & $\begin{array}{c}\text { Deviations } \\
\text { from the } \\
\text { definition of } \\
\text { the } \\
\text { denominator }\end{array}$ & $\begin{array}{l}\text { Deviations } \\
\text { from the } \\
\text { definition of } \\
\text { the numerator }\end{array}$ & $\begin{array}{c}\text { Additional } \\
\text { years } \\
\text { available }\end{array}$ & Comments \\
\hline & $\begin{array}{l}\text { Ministerio de Sanidad y } \\
\text { Consumo. Instituto de } \\
\text { Información Sanitaria. Registro } \\
\text { de altas } 2004\end{array}$ & & & & & & \\
\hline Sweden & $\begin{array}{l}\text { The Swedish Hospital Discharge } \\
\text { Register }\end{array}$ & & & & & & \\
\hline $\begin{array}{l}\text { United } \\
\text { States }\end{array}$ & $\begin{array}{l}\text { Healthy People 2010, measure } \\
24-2 \text { (modified age group). } \\
\text { Agency for Healthcare Research } \\
\text { and Quality (AHRQ) Prevention } \\
\text { Quality Indicators (PQ }\end{array}$ & & & $\begin{array}{l}\text { U.S. } \\
\text { population age } \\
18+\end{array}$ & $\begin{array}{l}\text { Number of } \\
\text { discharges } \\
\text { with first listed } \\
\text { diagnosis of } \\
\text { asthma (ICD- } \\
\text { 9-CM code } \\
\text { 493) among } \\
\text { adults age } 18 \\
\text { and over. } \\
\text { Excludes } \\
\text { obstetric } \\
\text { admissions } \\
\text { and transfers } \\
\text { from other } \\
\text { institutions }\end{array}$ & & $\begin{array}{l}\text { Rates are adjusted by age and gender, using the total U.S. } \\
\text { population for } 2000 \text { as the standard population. Although not } \\
\text { all States participate in the HCUP database, the Nationwide } \\
\text { Inpatient Sample is weighted to give national estimates using } \\
\text { weights based on all U.S. community, non-rehabilitation } \\
\text { hospitals in the American Hospital Association Annual Survey } \\
\text { of Hospitals. }\end{array}$ \\
\hline
\end{tabular}


DELSA/HEA/WD/HWP(2007)4

\begin{tabular}{|c|c|c|c|c|c|c|c|}
\hline \multicolumn{8}{|c|}{ Non-OECD EU countries } \\
\hline Country & Source & $\begin{array}{c}\text { Diagnosis } \\
\text { code(s) }\end{array}$ & $\begin{array}{l}\text { Age } \\
\text { groups } \\
\text { included }\end{array}$ & $\begin{array}{l}\text { Deviations } \\
\text { from the } \\
\text { definition of } \\
\text { the } \\
\text { denominator }\end{array}$ & $\begin{array}{l}\text { Deviations } \\
\text { from the } \\
\text { definition of } \\
\text { the numerator }\end{array}$ & $\begin{array}{l}\text { Additional } \\
\text { years } \\
\text { available }\end{array}$ & Comments \\
\hline Latvia & $\begin{array}{l}\text { Health statistics and medical } \\
\text { technology state agency }\end{array}$ & & & & $\begin{array}{l}\text { Numerator is } \\
\text { hospital } \\
\text { statistics rate, } \\
\text { not in primary } \\
\text { care. } \\
\text { Hospitalised } \\
\text { discharges of } \\
\text { age } 18 \text { years } \\
\text { and over with } \\
\text { asthma (ICD- } \\
10 \mathrm{~J} 45, \mathrm{~J} 46)\end{array}$ & & $\begin{array}{l}\text { At this moment, we have only information from branch } \\
\text { statistical report about discharged rate, but in the future it will } \\
\text { be able from the Management Information System. }\end{array}$ \\
\hline
\end{tabular}


DELSA/HEA/WD/HWP(2007)4

\section{INDICATORS NOT SELECTED FOR INCLUSION IN 2006 HCQI INDICATOR SET}

65. This section presents information on the clinical importance, scientific soundness together with specifications and data results for the 7 indicators which are not yet considered suitable for international comparisons by the HCQI Expert Group and which are still under review. The criteria applied for this assessment of the indicators were either the lack of availability of the indicator across a sufficient number of countries or some major divergences in the methodology of calculation between countries hampering seriously the comparability of figures. The comparability problems underlying this judgement are presented and possible solutions suggested. The section offers information about the 4 indicators already tested in the previous data collection for which the availability and comparability problems have not yet been satisfactorily solved and 3 new indicators tested for the first time in 2006 data collection, for which the HCQI Expert Group recommended further work before inclusion in the HCQI indicators set.

\section{Annual HbAlc test for patients with diabetes}

NOTE that this indicator was already tested in (2003-2005) HCQI data collection and it is not yet considered suitable for international comparisons by the HCQI Expert Group. It is included in this paper to illustrate current data concerns with the indicator and possible future solutions

\section{Operational Definition}

Numerator: Number of patients with at least one test of HbA1c levels in the reporting year.

Denominator: People age 18-75 with diabetes mellitus type I or II, defined as: at least one physician visit with a diagnosis of diabetes or patient dispensed insulin and/or hypoglycaemic agent, excluding those with gestational diabetes and those not seen for continuing care.

\section{Importance}

66. Mortality: Diabetes mellitus was responsible for an estimated 21 deaths per 100000 people in WHO Euro A countries in 2000. This represents $2.1 \%$ of all deaths.

67. Prevalence: Diabetes mellitus affected an estimated 3 of every 100 people living in WHO Euro A countries in 2000. Diabetes mellitus constitutes a major public health burden in the industrialised countries, affecting, for example in the US an estimated 15.7 million people, including an estimated 5.4 million people not yet diagnosed. In addition to being the seventh leading cause of death in the US (ADA, 2006), diabetes mellitus is also the leading cause of blindness in people ages 20-74, the leading cause of end-stage renal disease (ESRD), the most frequent cause of non-traumatic lower limb amputations, and a major risk factor for heart disease and stroke. The prevalence of diabetes in the US is projected to increase from the present rate of $5.9 \%$ to $8.9 \%$ by 2025 (ADA, 2006).

68. Cost: Endocrine diseases, of which diabetes is the most common, are the $12^{\text {th }}$ attributable contributor to cost of illness in Canada ( $2.2 \%$ of direct and indirect costs). In the United States, the costs of diabetes totalled $\$ 132$ billion in 2002, including about $\$ 92$ billion in direct medical expenditures and about $\$ 40$ billion in lost productivity and premature death (Hogan, 2003). 


\section{DELSA/HEA/WD/HWP(2007)4}

\section{Scientific Soundness}

69. Face validity: There is now strong evidence that reducing blood glucose to normal levels can reduce the risk of complications associated with both type I and type II (ADA, 2001) diabetes. Severity of complications associated with chronic diabetes, lack of symptoms in initial stages of disease, and long period between the commencement of sustained hyperglycaemia and observable complications make this disease a prime candidate for aggressive, outpatient based, primary preventive care. Blood glucose testing using $\mathrm{HbA} 1 \mathrm{c}$ is recommended as a quality indicator by the American Medical Association and is used by the US Veteran's Administration. The American Diabetes Association and many other national scientific societies have issued guidelines for the management of diabetes that reflect the implications of this research (ADA, 2001). These guidelines have been disseminated widely, and adapted by many health care provider organisations to reflect local practice. Yet as in other areas of clinical practice, numerous studies have documented that the level of clinician adherence to diabetes practice guidelines' recommendations for routine monitoring and screening remains variable and often quite low (Streja, 1999; Lawler, 1997).

70. Content validity: Reviews of the evidence from clinical trials of diabetes management, including those conducted by the Cochrane Collaboration, the American Diabetes Association, the New Zealand Guidelines Group, and others, have all concluded that good glycemic control reduces the occurrence of retinopathy, nephropathy, and neuropathy, and improves functional status and well-being among people with type I and type II diabetes (Renders, 2002; Nathan; 2002).

71. Reliability: Data on the frequency of $\mathrm{HbA} 1 \mathrm{c}$ testing is usually derived from studies using medical chart review or prospective data collection. International comparison of these studies is therefore affected by all differences in study design.

\section{Feasibility}

72. Data availability: HbAlc test rates are available for eight countries (Table 21). The HbAlc screening data supplied were for 2000-2005. Five countries provided data that slightly deviated from the requested OECD age range. Countries provided data based on samples from primary care clinics, and from patient surveys. Countries that reported from general surveys also reported a problem that many diabetics are not familiar with the term of $\mathrm{HbAc} 1$.

73. Comparability issues: Detailed documentation and assessment is provided in Table 22. There should be concern over comparing the results of patient surveys to a review of patient records. A major challenge for this indicator relates to fielding surveys that might be able to accurately collect information on $\mathrm{HbAcl}$ testing. Because diabetics are not always familiar with the term $\mathrm{HbAc1}$, self-reported data may not be reliable, resulting in one country not reporting their data. Data derived as part of research project may not be generalisable to a country, because care patterns and patient characteristics may be systematically different from the general population.

74. Overall assessment: Only eight countries could provide data on this indicator. It also appears that some of the data comes from research projects and may not be regularly collected. However, during the course of the project, data availability improved for this indicator and it may warrant examination in future HCQI efforts. 
Table 21. Annual HbA1c test for patients with diabetes

\begin{tabular}{|l|c|c|c|}
\hline \multicolumn{1}{|c|}{ Country } & $\begin{array}{c}\text { Diabetic } \\
\text { patients tested } \\
\text { for HbA1c in the } \\
\text { last year (\%) }\end{array}$ & Data year & Age \\
\hline Finland & 98.0 & 2000 & \\
\hline France & 82.6 & 2002 & $>=18$ years \\
\hline Italy & Type I 91.0 & $14+$ \\
\hline Norway & 2004 & $14+$ \\
\hline Spain & 93.0 & 2000 & \\
\hline Sweden & 77.4 & 2000 & $16+$ \\
\hline United Kingdom & 97.0 & 2003 & 18 and over \\
\hline United States & 94.4 & $2004-2005$ & 2002 \\
\hline
\end{tabular}


DELSA/HEA/WD/HWP(2007)4

\section{Annual HbAlc test for patients with diabetes, sources and methods}

\begin{tabular}{|c|c|c|c|}
\hline Country & Source & $\begin{array}{l}\text { Age groups } \\
\text { included }\end{array}$ & Comments \\
\hline Finland & $\begin{array}{l}\text { A survey conducted in } \\
2000\end{array}$ & & $\begin{array}{l}\text { Data come from a research project, and are a representative sample of diabetics in Finland. The objective was to } \\
\text { describe the level of diabetic care in Finland. The criteria were } \mathrm{HbA} 1 \mathrm{c} \text { levels, blood pressure and lipid level. } 3580 \\
\text { diabetic took part, } 3462 \text { had had their } \mathrm{HbAc} 1 \text { level measured. The results are } 97 \% \text { in patients on oral medication, } 99 \% \text { in } \\
\text { patients on insulin, and } 100 \% \text { in patients on combination medication. }\end{array}$ \\
\hline France & $\begin{array}{l}\text { "Entred" (survey based } \\
\text { upon a national sample } \\
\text { of diabetic patients } \\
\text { whose health } \\
\text { insurance is "Caisse } \\
\text { nationale des } \\
\text { travailleurs salariés") }\end{array}$ & $18+$ & Patients repaid for insulin or hypoglycaemic agents \\
\hline Italy & $\begin{array}{l}\text { Associazone medici } \\
\text { diabetologi }\end{array}$ & $1-100$ & $\begin{array}{l}\text { Based on sample of } 120.000 \text { diabetic persons of any age. The information has been derived from electronic records of } \\
86 \text { diabetes outpatient clinics. In Italy } 50-70 \% \text { patients are followed by diabetes clinics }\end{array}$ \\
\hline Norway & $\begin{array}{l}\text { Unpublished data from } \\
\text { an epidemiological } \\
\text { study carried out in two } \\
\text { parts of Norway }\end{array}$ & & $\begin{array}{l}\text { A sample of } 2000 \text { patients with diabetes attending primary care in Norway had their HbA1c tested at least once during } \\
\text { the year }\end{array}$ \\
\hline Spain & $\begin{array}{l}\text { GEDAPS (Study Group } \\
\text { of Diabetes in Primary } \\
\text { Health Care) }\end{array}$ & $14+$ & $\begin{array}{l}\text { This indicator was based in a sample of } 6202 \text { people with diabetes mellitus aged } 14 \text { years and older. This sample was } \\
\text { obtained from the morbidity registries in several centres of primary health care by physicians who participate voluntarily } \\
\text { in a program to improve care quality }\end{array}$ \\
\hline Sweden & $\begin{array}{l}\text { National Diabetic } \\
\text { Register, covering } \\
\text { approximately } 30 \% \text { of } \\
\text { all diabetics in Sweden }\end{array}$ & & $\begin{array}{l}\text { In Sweden the focus has shifted to monitoring evidence-based practice and outcomes of care. Only patients with type I } \\
\text { diabetes or type II diabetes and at least one test of HbA1c levels were reported this year } 2003 .\end{array}$ \\
\hline $\begin{array}{l}\text { United } \\
\text { Kingdom }\end{array}$ & $\begin{array}{l}\text { Quality and Outcomes } \\
\text { Framework (QOF), } \\
\text { Health and Social Care } \\
\text { Information Centre }\end{array}$ & $16+$ & $\begin{array}{l}\text { The data provided represents "the percentage of diabetic patients who have a record of HbA1c or equivalent in the } \\
\text { previous } 15 \text { months. As the care of children with diabetes mellitus is generally under the control of specialists, the } \\
\text { register should exclude those patients age } 16 \text { and under. Likewise, the indicators are not intended to apply to patients } \\
\text { with gestational diabetes and relate to patients with both type I and type II diabetes. Data does not adjust for age or } \\
\text { gender-they are crude rates. No allowance is made for e.g. deprivation and ethnicity. And importantly, there are } \\
\text { "exclusions" from QOF e.g. if a patient fails to show for repeat requests for annual review, GPs can and do exclude them } \\
\text { from the denominator. }\end{array}$ \\
\hline $\begin{array}{l}\text { United } \\
\text { States }\end{array}$ & MEPS & $18+$ & $\begin{array}{l}\text { Research for the Medical Expenditure Panel Survey (MEPS) at US DHHS AHRQ has shown that there are a large } \\
\text { number of non-respondents to questions about whether the individual had an HbA1C test due to lack of knowledge } \\
\text { about } \mathrm{HbA} 1 \mathrm{C} \text {. }\end{array}$ \\
\hline
\end{tabular}


Table 22. Annual HbA1c test for patients with diabetes, comparability issues

\begin{tabular}{|c|c|c|c|}
\hline & & \multicolumn{2}{|c|}{ Comparability Implications } \\
\hline & & Minor & Severe \\
\hline & Possible & Age ranges vary & \\
\hline 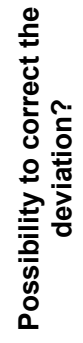 & Unlikely & Data available for different years & $\begin{array}{l}\text { Diabetics often are unfamiliar with the } \\
\text { term "HbAc1" leading to potential bias } \\
\text { in population surveys. } \\
\text { Comparability between } \\
\text { population/patient surveys and review } \\
\text { of patient records is unknown. } \\
\text { Data derived from research studies } \\
\text { may not be generalisable }\end{array}$ \\
\hline
\end{tabular}

Possible solutions:

- Footnotes can indicate the year and age range

- OECD could investigate the comparability between in-person surveys and a review of patient records.

- Tables should separate results based on population-level data and research studies as well as those based on survey data and patient records. 


\section{HbAlc level indicating poor glucose control}

NOTE that this indicator was already tested in (2003-2005) HCQI data collection and it is not yet considered suitable for international comparisons by the HCQI Expert Group. It is included in this paper to illustrate current data concerns with the indicator and possible future solutions.

\section{Operational Definition}

Numerator: Number of patients with $\mathrm{HbAlc}$ level greater than $9.5 \%$ at the most recent test given in the reporting year.

Denominator: People age 18-75 with diabetes mellitus type I or II who had HbA1c levels tested within the reporting year (Diabetes defined as: at least one physician visit with a diagnosis of diabetes OR patient dispensed insulin and/or hypoglycaemic agent, excluding those with gestational diabetes).

Importance (for a more detailed discussed, please see above under HbAlc test rate)

75. Mortality: Diabetes mellitus was responsible for an estimated 21 deaths per 100000 people in WHO Euro A countries in 2000. This represents $2.1 \%$ of all deaths.

76. Prevalence: Diabetes mellitus affected an estimated three of every 100 people living in WHO Euro A countries in 2000.

77. Cost: Endocrine diseases, of which diabetes is the most common, are the $12^{\text {th }}$ highest contributor to cost of illness in Canada (2.2\% of direct and indirect costs). In the United States, the costs of diabetes totalled $\$ 132$ billion in 2002, including about $\$ 92$ billion in direct medical expenditures and about $\$ 40$ billion in lost productivity and premature death (Hogan, 2003).

\section{Scientific Soundness}

78. Face validity: $\mathrm{HbAlc}$ has been termed the memory of glucose control. Chronically elevated blood glucose levels, indicating poor glycemic control, lead to chemical alterations of the haemoglobin, the component of the red blood cells that transport oxygen. By measuring HbAlc-levels, clinicians gain insight into the glycemic control of a patient over the last couple of weeks. Thus, the test determines how well a patient's diabetes has been managed with elevated values indicating uncontrolled diabetes.

79. Content validity: Reviews of the evidence from clinical trials of diabetes management, including those conducted by the Cochrane Collaboration, the American Diabetes Association, the New Zealand Guidelines Group, and others, have all concluded that good glycemic control reduces the occurrence of retinopathy, nephropathy, and neuropathy, and improves functional status and well-being among people with type I and type II diabetes (Renders, 2002; Nathan, 2003). Many diabetic patients have poor glycemic control (Renders, 2002). The threshold for this indicator, 9.5\% (indicating very poor glycemic control), is based on a recommendation from a group of 15 experts in developing clinical diabetes indicators for the National Committee for Quality Assurance (NCQA, 2003). This threshold, however, will need to be updated periodically as numerous organisations, including the US National Quality Forum and the Alliance on Diabetes Quality Improvement (representing the American Medical Association, the American Diabetes Association and the Joint Commission on the Accreditation of Healthcare Organisations) have updated this threshold to a more stringent level. 
80. Reliability: Different HbA1c tests could provide different results. However, the threshold chosen was judged high enough so that no patient, regardless of the test used or health condition should exceed the threshold.

\section{Feasibility}

81. Data availability: HbA1c levels are available for eleven countries (Table 23). The screening data are supplied for a range of years. Some countries provided data that slightly deviated from the OECD age range requested. Some countries used population surveys, and others sampled from clinics or hospitals. One country used a sample from a specialty clinic, which may not be representative of diabetes care nationally. One country provided data with a definition that was significantly more rigorous than the OECD definition. (Although another country, while supplying the data as requested, regularly uses the more rigorous target as well.) One country provided data for a specific ethnic group that is not generalisable to the national level.

82. Comparability issues: Detailed documentation and assessment is provided in Table 24. The differences in years provided at age deviations do not appear to be significant threats to validity. The variation in definition of poor glucose control ( $\mathrm{HbAc} 1>9.5 \%)$ is a significant problem with respect to international comparability. The differing sampling techniques are likely to pose threats to comparability.

83. Overall assessment: Eleven countries could provide data on this indicator. It appears that some of the data stem from research studies and may not be regularly collected. Data would be available in patient records, but would require a review of patient records which currently is not routinely done in most national data collection systems.

Table 23. HbA1c level indicating poor glucose control

\begin{tabular}{|c|c|c|c|}
\hline Country & $\begin{array}{c}\text { Diabetic patients with HbA1c } \\
\text { levels }>9.5 \%\end{array}$ & Data year & Age \\
\hline Australia & 10.9 & $1999-2000$ & $25-75$ \\
\hline Finland & $\begin{array}{l}17.7 \\
\text { type } 1 \text { diabetics: } 28.1 \\
\text { type } 2 \text { diabetics: } 12.5\end{array}$ & 2000 & \\
\hline France & $\begin{array}{c}\text { missing: } 7.9 \\
\text { <=6.5: } 26.6 \\
\text { [6.5-8]: } 40.3 \\
\text { [8-10]: } 20.9 \\
\text { >10: } 4.3\end{array}$ & 2001 & \\
\hline Germany & 16.4 & 1998 & $18-75$ \\
\hline Italy & 10.7 & 2003 & $14+$ \\
\hline Mexico & 20.8 & 2002 & $18-75$ \\
\hline New Zealand & 9.9 & 2001 & $18-75$ \\
\hline Spain & 9.5 & 2000 & $14+$ \\
\hline Sweden & $\begin{array}{c}\text { PHC } 60.0 \\
\text { Hospital Clinics } 31.0\end{array}$ & 2001 & \\
\hline United Kingdom & 10.6 & 2004-2005 & $16+$ \\
\hline United States & 21.0 & 1999-2002 & $18+$ \\
\hline
\end{tabular}


DELSA/HEA/WD/HWP(2007)4

HbAlc level indicating poor glucose control, sources and methods

\begin{tabular}{|c|c|c|c|}
\hline Country & Source & $\begin{array}{c}\text { Age } \\
\text { groups } \\
\text { included }\end{array}$ & Comments \\
\hline Australia & $\begin{array}{l}\text { Australian Diabetes Obesity, } \\
\text { and Lifestyle Study (AusDiab) }\end{array}$ & $25-75$ & Data were weighted to match the age and sex distribution of the 1998 residential population of Australia aged 25 and older. \\
\hline Finland & $\begin{array}{l}\text { A survey among Finnish } \\
\text { diabetics (a representative } \\
\text { sample of } 3580 \text {, of whom } 3462 \\
\text { had their HbAc1 measured) }\end{array}$ & & \\
\hline France & $\begin{array}{l}\text { "Entred" (survey based upon a } \\
\text { national sample of diabetic } \\
\text { patients whose health } \\
\text { insurance is "Caisse nationale } \\
\text { des travailleurs salariés") }\end{array}$ & & $\begin{array}{l}\text { Sample of } 1718 \text { patients repaid for insulin or hypoglycaemic agents: a questionnaire was sent to patients and then another to their } \\
\text { practitioner }\end{array}$ \\
\hline Germany & $\begin{array}{l}\text { German National Health } \\
\text { Interview and Examination } \\
\text { Survey } 1998 \text { (Bundes- } \\
\text { Gesundheitssurvey 1998) }\end{array}$ & $18-75$ & The result is based on data of a population survey, which included 298 diabetic persons in accordance with the definitions stated above. \\
\hline Italy & Study "SFIDA" & $35-70$ & $\begin{array}{l}\text { Information on metabolic control comes from cross-sectional study involving } 12222 \text { patients with type } 2 \text { DB enrolled by } 261 \text { DB outpatient } \\
\text { clinics (more than } 1 \text { third of Italian DB outpatient clinics). Data refers to individuals with levels }>8 \% \mathrm{HbA} 1 \mathrm{c} \text {. }\end{array}$ \\
\hline $\begin{array}{l}\text { New } \\
\text { Zealand }\end{array}$ & Annual Check Program & $18-75$ & NZ uses proportion with HBA1c $>8 \%$ as a performance indicator for District Health Boards. This is reported, and targets are set, by ethnicity \\
\hline Spain & $\begin{array}{l}\text { GEDAPS (Study Group of } \\
\text { Diabetes in Primary Health } \\
\text { Care) }\end{array}$ & $14+$ & $\begin{array}{l}\text { This indicator was based in a sample of } 6202 \text { people with diabetes mellitus aged } 14 \text { years and older. This sample was obtained from the } \\
\text { morbidity registries in several centres of primary health care by physicians who participated voluntarily in a program to improve care quality. }\end{array}$ \\
\hline Sweden & National Diabetes Register & & $\begin{array}{l}\text { The difference between PHC and hospital clinics is likely to depend on patient selection. The measure can be reported, e.g., per type of } \\
\text { diabetes, age and sex. }\end{array}$ \\
\hline $\begin{array}{l}\text { United } \\
\text { Kingdom }\end{array}$ & $\begin{array}{l}\text { Quality and Outcomes } \\
\text { Framework (QOF), Health and } \\
\text { Social Care Information Centre }\end{array}$ & $16+$ & \\
\hline $\begin{array}{l}\text { United } \\
\text { States }\end{array}$ & $\begin{array}{l}\text { National Health and Nutrition } \\
\text { Examination Survey } \\
\text { (NHANES), NHQR }\end{array}$ & $18+$ & Non-institutionalised diagnosed diabetics \\
\hline
\end{tabular}


Table 24. HbA1c level indicating poor glucose control, comparability issues

\begin{tabular}{|c|c|c|c|}
\hline & & \multicolumn{2}{|c|}{ Comparability Implications } \\
\hline & & Minor & Severe \\
\hline 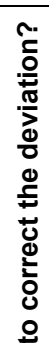 & Possible & $\begin{array}{l}\text { 1. Age ranges vary } \\
\text { 2. Data available for different years }\end{array}$ & $\begin{array}{l}\text { 1. Data provided for different definition } \\
\text { of poor glucose control }(\mathrm{HbAc} 1>8 \% \text {, } \\
\text { compared to } \mathrm{HbAc1}>9.5 \%) \\
\text { 2. Some countries obtain samples from } \\
\text { population based surveys and some } \\
\text { from specialised clinics. The } \\
\text { generalisability of such selected } \\
\text { samples is unknown. }\end{array}$ \\
\hline 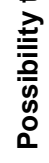 & Unlikely & & $\begin{array}{l}\text { 1. Some countries obtain samples from } \\
\text { population based surveys and some } \\
\text { from specialised clinics. The } \\
\text { generalisability of such selected } \\
\text { samples is unknown. }\end{array}$ \\
\hline
\end{tabular}

Possible solutions:

- Footnotes can indicate the year and age range

- In the future, OECD can work with countries to provide data that is consistent with HCQI definition of poor control.

- Drop or report separately data from countries that cannot provide data that is generalisable to the national level. 
DELSA/HEA/WD/HWP(2007)4

\section{Major amputation in diabetics}

NOTE that this indicator was already tested in (2003-2005) HCQI data collection and it is not yet considered suitable for international comparisons by the HCQI Expert Group. It is included in this paper to illustrate current data concerns with the indicator and possible future solutions.

\section{Operational Definition}

Numerator: Number of diabetic patients with major (above or below knee) amputations in a given year.

Denominator: Number of patients with diabetes (type I and type II) ages 18-75 years.

\section{Importance $^{25}$}

84. Mortality: Diabetes mellitus was responsible for an estimated 21 deaths per 100,000 people in WHO Euro A countries in 2000. This represents $2 \%$ of all deaths.

85. Prevalence: Each year, more than 10,000 Americans with diabetes face decisions related to amputation. Two of the main complications of longstanding inadequate glycemic control (indicating poor diabetes management) are peripheral vascular disease, the chronic deprivation of blood supply of the legs due to arteriolosclerosis, and peripheral neuropathy, damage to the peripheral nervous system. The combination of those two complications put diabetics at greater risk for lower extremity lesions. Loss of sensation in the foot increases likelihood that minor trauma goes unnoticed, while inadequate blood supply results in impaired healing of the wound and greater risk of infection. Thus, osteomyelitis (severe infections of the bone) and gangrene (infection induced tissue necrosis) may result. For about $75 \%$ of the cases, a partial amputation of a foot may be enough to stop the foot ulcer from progressing, but for the remaining $25 \%$, it will be necessary to remove the leg from below the knee (Mundell, 2004). Diabetics are also at higher risk of developing uninfected necroses of the lower extremities because of vascular complications. In the US, minority populations have had the highest rates of amputations and it is thought that socioeconomic status is a major factor leading to amputations. Thus, differences in level and distribution of wealth may be reflected in the measure together with differences in quality of care.

86. Cost: Amputations have a large impact on health, particularly on quality of life, and result in substantial follow-up cost in the form of rehabilitation, prostheses and disability.

\section{Scientific Soundness}

87. Face validity: Adequate glycemic control has been shown to reduce the risk and severity of neuropathy and vascular complications in diabetics (Renders, 2002). It is also widely believed that careful monitoring for an intensive treatment of minor lesions in the presence of neuropathic and arterial disease of the extremities can prevent amputations, but only a few randomised trials have been conducted to support this (Lavery, 2000).

88. Construct validity: The main challenge to the construct validity of this indicator is a certain disjoint of the underlying concept and the operationalisation. Precisely speaking the concept behind the indicator is that proper diabetes management should reduce the risk of severe tissue damage to lower extremities. However, the indicator measures amputation rates, a closely related but slightly different

\footnotetext{
${ }^{25}$ For additional discussion on the importance of diabetes, please refer to the discussion above under the retinal exam indicator
} 
concept that captures the typical consequence of severe tissue damage. One could also argue that, while severe tissue damage is unambiguously negative, the decision to amputate is not so that the indicator does not clearly indicate better or worse quality of care. However, many regard major amputation rates as reasonable proxy for severe tissue damage rates and thus a valid quality indicator. ${ }^{26}$ Because of the importance of this complication and plausibility of the concept behind the indicator, this measure has great potential. But it needs to be further studied before adopting it for international comparisons.

89. Reliability: As this indicator is derived from hospital discharge information, the ability to construct it reliably for international comparisons depends on the comparability of coding and reporting practices across countries. Amputation rates should be ascertainable in a reliable fashion in administrative data, as is done currently in the US by the Centers for Disease Control and Prevention (NCQA, 2003), because such major procedures usually influence hospital payments and are thus reliably reported. But it may be difficult to reliably identify the diabetic population, because diabetes may only be recorded as comorbid condition rather than the primary reason for admission and coding of such secondary diagnoses may vary across countries.

\section{Feasibility}

90. Data availability: Major amputations in diabetics' rates are available for 14 countries (Table 25). The data were reported for years ranging from 1994 to 2004. The OECD definition is for all ages, and five countries had deviations in the age range. All countries use hospital records for the numerator, but the method of estimating the denominator varied. Some countries used previous estimates or population surveys to obtain the denominators. Other countries used administrative data to obtain the denominator, which would not capture all diabetics but only the diabetics receiving hospital or other medical care, and the diagnostic codes to capture the diabetic population varied. One country indicated that hospital records may be incompletely coded, and may underestimate the amputations on diabetics. Countries used different procedure codes, and even some using the same coding system included different (more or less) procedures. Countries used varying inclusion criteria for the procedure, even accounting for differences in national coding systems. This is of serious concern to comparability.

91. Comparability issues: Detailed documentation and assessment is provided below. There are serious concerns about comparability, both from the estimation of the denominator, and because different procedures are being included, the HCQI project will have to ensure that countries are reporting the same type of amputations.

92. Overall assessment: Fourteen countries provided data on this indicator. While information for this indicator might exist in hospital records of other countries as well, it is unclear how many countries would be able to construct this indicator on a routine basis. Additionally, a significant amount of analytic work will have to be done in order to ensure that the data are internationally comparable. However, data comparability and availability for this indicator improved during the project and the indicator may warrant examination in the future as part of HCQI indicator updates.

\footnotetext{
${ }^{26}$ The situation is clearly different for minor amputations (e.g., toes), where timely amputations can avoid progression of the disease.
} 
Table 25. Major amputation in diabetics, per 10000 diabetics

\begin{tabular}{|l|c|c|}
\hline \multicolumn{1}{|c|}{ Country } & $\begin{array}{c}\text { Incidence of } \\
\text { amputations } \\
\text { per 10 000 }\end{array}$ & Data year \\
\hline Australia & 6.3 & $1999-2000$ \\
\hline Austria & 13.2 & 2004 \\
\hline Canada & 8.5 & $1999-2000$ \\
\hline Czech Republic & 106.3 & 2005 \\
\hline Finland & 27.8 & 2005 \\
& 7.0 & 2004 \\
& 5.0 & 2002 \\
\hline France & 15.0 & 2001 \\
\hline Italy & 0.1 & 2004 \\
\hline Mexico & 11.8 & 2005 \\
& 7.9 & 2003 \\
\hline Netherlands & 7.8 & 2004 \\
& 35.0 & 2000 \\
\hline New Zealand & 14.6 & $2005-2006$ \\
& 68.0 & $2002-2003$ \\
\hline Norway & 50.0 & 1994 \\
\hline Portugal & 40.0 & 2004 \\
& 51.0 & 2002 \\
\hline Slovak Republic & 138.0 & 2004 \\
\hline Spain & 18.4 & 2004 \\
\hline Sweden & 101.2 & 2005 \\
& 87.0 & 2003 \\
\hline United Kingdom & 17.8 & $2003-2004$ \\
& 23.0 & $2002-2003$ \\
\hline United States & 44.0 & $2002-2004$ \\
\hline & 56.0 & $1999-2001$ \\
\hline
\end{tabular}

\begin{tabular}{|l|c|c|}
\hline \multicolumn{3}{|c|}{ Non-OECD EU countries } \\
\hline Country & $\begin{array}{c}\text { Incidence of } \\
\text { amputations } \\
\text { per 10 000 }\end{array}$ & Data year \\
\hline Latvia & 62.8 & 2005 \\
\hline
\end{tabular}


Major amputation in diabetics, sources and methods

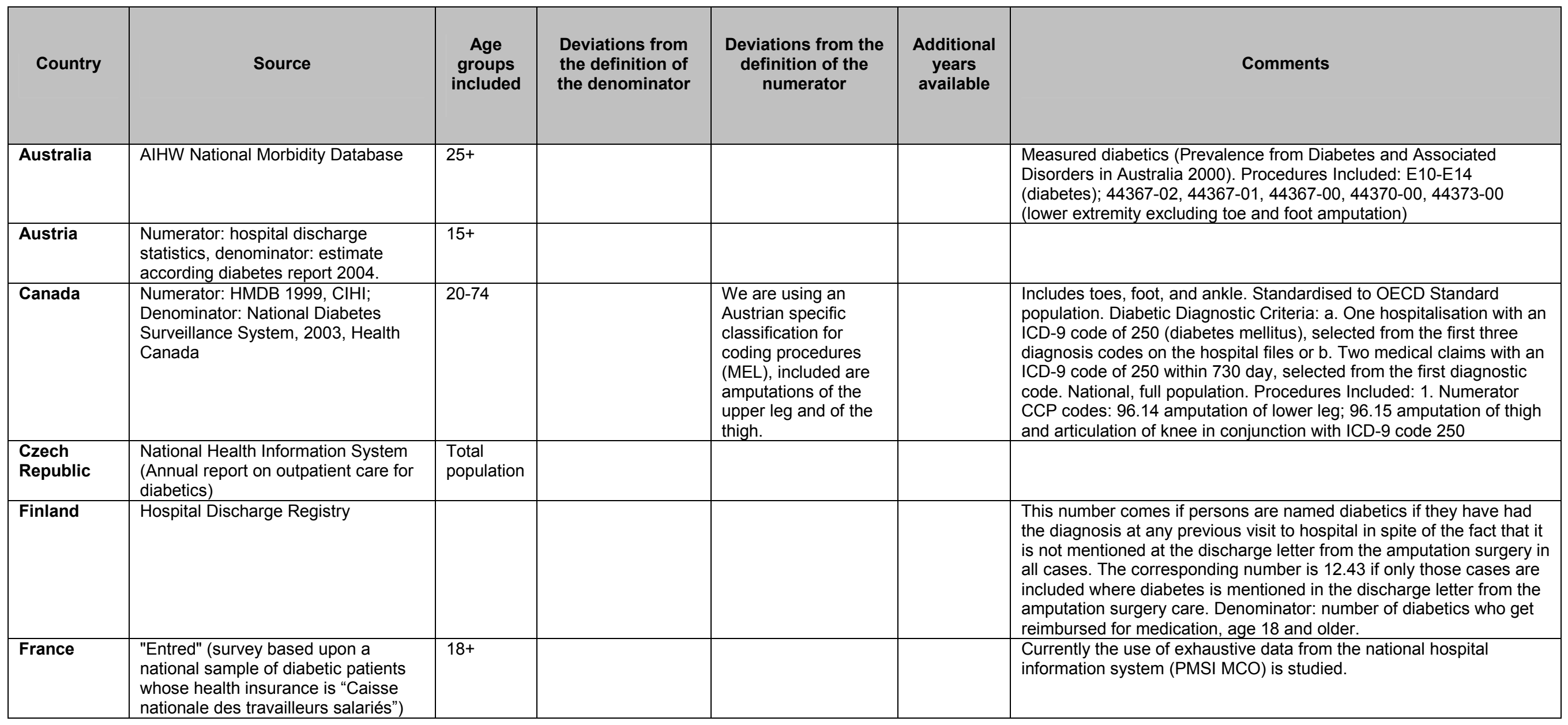


DELSA/HEA/WD/HWP(2007)4

\begin{tabular}{|c|c|c|c|c|c|c|}
\hline Country & Source & $\begin{array}{l}\text { Age } \\
\text { groups } \\
\text { included }\end{array}$ & $\begin{array}{l}\text { Deviations from } \\
\text { the definition of } \\
\text { the denominator }\end{array}$ & $\begin{array}{l}\text { Deviations from the } \\
\text { definition of the } \\
\text { numerator }\end{array}$ & $\begin{array}{l}\text { Additional } \\
\text { years } \\
\text { available }\end{array}$ & Comments \\
\hline Italy & Ministry of Health / ISTAT & & $\begin{array}{l}\text { Main procedure } \\
\text { codes: } 84.15 ; \\
84.17 \text {. All the major } \\
\text { amputations rates } \\
\text { for the years } 2002- \\
2004 \text { are expressed } \\
\text { per } 10000 \\
\text { diabetics. The } \\
\text { denominator source } \\
\text { is the National } \\
\text { hospital discharges } \\
\text { database for the } \\
\text { years } 2002-2003 \\
\text { and for the year } \\
2004 \text { it is the multi- } \\
\text { purpose survey on } \\
\text { "health status of the } \\
\text { population and use } \\
\text { of health services" } \\
\text { conducted by the } \\
\text { National Institute of } \\
\text { Statistics. }\end{array}$ & $\begin{array}{l}\text { The numerator } \\
\text { represents the } \\
\text { number of the in- } \\
\text { patients, being no } \\
\text { less } 18 \text { years old, } \\
\text { with the main surgical } \\
\text { procedure "84.15" or } \\
\text { "84.17" by ICD-9-CM } \\
\text { and having the codes } \\
\text { " } 250.7^{\star \prime} \text { as main } \\
\text { diagnosis and at least } \\
\text { one secondary } \\
\text { diagnosis with the } \\
\text { code " } 443.81 \text { " by } \\
\text { ICD-9-CM }\end{array}$ & $2001+$ & $\begin{array}{l}\text { The denominator used for this indicator has been modified. It has } \\
\text { been supplied by the National Institute of Statistics and it represents } \\
\text { the estimate of the Italian people suffering from diabetes. } \\
\text { Multipurpose survey on "health status of the population and use of } \\
\text { health services" National Institute of Statistics }\end{array}$ \\
\hline Mexico & $\begin{array}{l}\text { Sistema de Información de Atención } \\
\text { Integral a la Salud SIAIS-IMSS. } \\
\text { División Técnica de Información } \\
\text { Estadística en Salud (DTIES) }\end{array}$ & & $\begin{array}{l}\text { Population estimate } \\
\text { based on the data } \\
\text { include only once } \\
\text { Diabetic patients } \\
\text { type I and II over } 20 \\
\text { years that had at } \\
\text { least one } \\
\text { consultation during } \\
2005 \text {. }\end{array}$ & $\begin{array}{l}\text { Yes, the age included } \\
\text { is Intervals are } 20 \\
\text { years and older. }\end{array}$ & & $\begin{array}{l}\text { Numerator includes ICD- } 984.10,84.15 \text { and } 84.17 \text { codes and these } \\
\text { are all de procedures effectuated during } 2005 \text { in the diabetic } \\
\text { population. }\end{array}$ \\
\hline
\end{tabular}


DELSA/HEA/WD/HWP(2007)4

\begin{tabular}{|c|c|c|c|c|c|c|}
\hline Country & Source & $\begin{array}{c}\text { Age } \\
\text { groups } \\
\text { included }\end{array}$ & $\begin{array}{l}\text { Deviations from } \\
\text { the definition of } \\
\text { the denominator }\end{array}$ & $\begin{array}{l}\text { Deviations from the } \\
\text { definition of the } \\
\text { numerator }\end{array}$ & $\begin{array}{l}\text { Additional } \\
\text { years } \\
\text { available }\end{array}$ & Comments \\
\hline Netherlands & $\begin{array}{l}\text { National Medical Registry, owned by } \\
\text { PRISMANT. Calculations and } \\
\text { methods by Statistics Netherlands in } \\
\text { cooperation with Institute for Public } \\
\text { Health and the Environment }\end{array}$ & $18+$ & $\begin{array}{l}\text { number of diabetes } \\
\text { patients, registered } \\
\text { by GPs. }\end{array}$ & $\begin{array}{l}\text { Number of patients } \\
\text { discharged in a one- } \\
\text { year period, with a } \\
\text { primary or secondary } \\
\text { diagnosis of diabetes } \\
\text { mellitus, and who had } \\
\text { an amputation of the } \\
\text { leg (Amputation } \\
\text { through tibia/fibula or } \\
\text { femur). }\end{array}$ & & 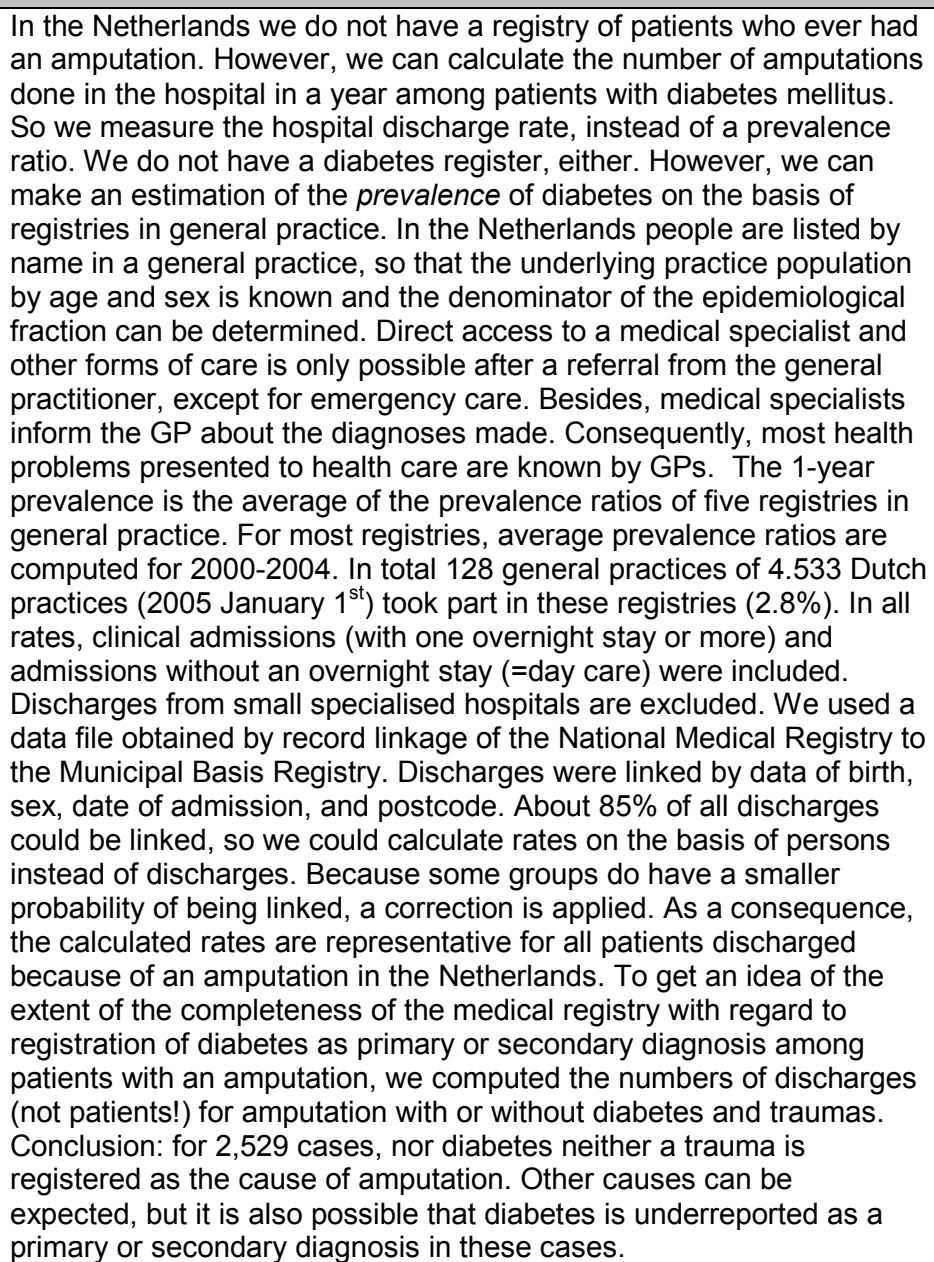 \\
\hline
\end{tabular}


DELSA/HEA/WD/HWP(2007)4

\begin{tabular}{|c|c|c|c|c|c|c|}
\hline Country & Source & $\begin{array}{l}\text { Age } \\
\text { groups } \\
\text { included }\end{array}$ & $\begin{array}{l}\text { Deviations from } \\
\text { the definition of } \\
\text { the denominator }\end{array}$ & $\begin{array}{l}\text { Deviations from the } \\
\text { definition of the } \\
\text { numerator }\end{array}$ & $\begin{array}{l}\text { Additional } \\
\text { years } \\
\text { available }\end{array}$ & Comments \\
\hline $\begin{array}{l}\text { New } \\
\text { Zealand }\end{array}$ & $\begin{array}{l}\text { National Minimum Data Set (NMDS) } \\
2005-2006\end{array}$ & & $\begin{array}{l}\text { An estimate based } \\
\text { on the 2002-2003 } \\
\text { New Zealand } \\
\text { Health Survey and } \\
\text { an assumed } 4 \% \\
\text { annual increment. }\end{array}$ & $\begin{array}{l}\text { Major amputation is } \\
\text { defined as an } \\
\text { amputation below } \\
\text { knee (44367-02), } \\
\text { disarticulation at knee } \\
\text { (44367-01), } \\
\text { amputation above } \\
\text { knee (44367-00), } \\
\text { amputation at hip } \\
\text { (44370-00) and } \\
\text { hindquarter } \\
\text { amputation (44373- } \\
00) \text {. }\end{array}$ & & Publicly funded events. \\
\hline Norway & $\begin{array}{l}\text { Hospital records in the form of patient } \\
\text { journals and operation theatre } \\
\text { protocols, compared to national } \\
\text { statistics on amputations, from } 4 \\
\text { counties. The county records show } \\
\text { that the national statistics. (from the } \\
\text { NPR, Norwegian Patient Register) } \\
\text { are of very high quality. The national } \\
\text { statistics for 1994, the only year } \\
\text { reviewed in detail, showed } 94-98 \% \text { of } \\
\text { the actual amputations performed on } \\
\text { diabetics in } 4 \text { counties. }\end{array}$ & $18+$ & & & & $\begin{array}{l}\text { Criteria: ICD-10 codes. Regional population, generalisable to nation. } \\
\text { Procedures Included: ICD9-250 + procedure code O 8716-19- } \\
\text { Diabetes+non-traumatic major amputation. }\end{array}$ \\
\hline Portugal & $\begin{array}{l}\text { Hospital discharges-- annual data } \\
\text { from diagnosis related groups } \\
\text { (DRGs) }\end{array}$ & $19-74$ & & & & $\begin{array}{l}\text { Only the data from the hospitals belonging to the National Health } \\
\text { Services are included. Discharges with principal and associated } \\
\text { diagnosis-- Diabetes Mellitus, code 250- IDC9-CM. National, full } \\
\text { population. Procedures Included: ICD9.CM code } 84.1\end{array}$ \\
\hline $\begin{array}{l}\text { Slovak } \\
\text { Republic }\end{array}$ & $\mathrm{NHIC}$ & & & & & \\
\hline Spain & $\begin{array}{l}\text { Numerator: Hospital Discharge } \\
\text { Minimum Data Set (Conjunto Mínimo } \\
\text { Básico de Datos CMBD). Ministerio } \\
\text { de Sanidad y Consumo. Instituto de } \\
\text { Información Sanitaria. Registro de } \\
\text { altas } 2004 \text {. Denominator: Health } \\
\text { Interview Survey 2003. }\end{array}$ & All & & $\begin{array}{l}\text { Numerator coding: } \\
\text { ICD-9-Diag } 250 \text { (any } \\
\text { diagnostic) + } \\
\text { procedure codes } 84 \\
15 \text { or } 84,16 \text { or } 84,17\end{array}$ & & $\begin{array}{l}\text { Discharges cover public hospital only ( } 75 \% \text { of total country } \\
\text { discharges). Needs to be adjusted by others risk factors: age. Other } \\
\text { data: by sex (male: } 24.27 \text {, female: } 13.4 \text { ) }\end{array}$ \\
\hline
\end{tabular}


DELSA/HEA/WD/HWP(2007)4

\begin{tabular}{|c|c|c|c|c|c|c|}
\hline Country & Source & $\begin{array}{l}\text { Age } \\
\text { groups } \\
\text { included }\end{array}$ & $\begin{array}{l}\text { Deviations from } \\
\text { the definition of } \\
\text { the denominator }\end{array}$ & $\begin{array}{l}\text { Deviations from the } \\
\text { definition of the } \\
\text { numerator }\end{array}$ & $\begin{array}{l}\text { Additional } \\
\text { years } \\
\text { available }\end{array}$ & Comments \\
\hline Sweden & $\begin{array}{l}\text { National Diabetic Register, covering } \\
\text { approximately } 30 \% \text { of all diabetics in } \\
\text { Sweden }\end{array}$ & $18-75$ & & & & $\begin{array}{l}\text { Sweden does not differentiate between Type I and Type II diabetes in } \\
\text { the Register. National, representative sample. }\end{array}$ \\
\hline $\begin{array}{l}\text { United } \\
\text { States }\end{array}$ & $\begin{array}{l}\text { Numerator: National Hospital } \\
\text { Discharge Survey, denominator: } \\
\text { National Health Interview Survey. } \\
\text { CDC NCHS National Hospital } \\
\text { Discharge Survey }\end{array}$ & All & & & & $\begin{array}{l}\text { US civilian persons who report that they have ever been diagnosed } \\
\text { with diabetes. National, representative sample }\end{array}$ \\
\hline
\end{tabular}


DELSA/HEA/WD/HWP(2007)4

\begin{tabular}{|c|c|c|c|c|c|c|}
\hline \multicolumn{7}{|c|}{ Non-OECD EU countries } \\
\hline Country & Source & $\begin{array}{l}\text { Age } \\
\text { groups } \\
\text { included }\end{array}$ & $\begin{array}{l}\text { Deviations from } \\
\text { the definition of } \\
\text { the denominator }\end{array}$ & $\begin{array}{l}\text { Deviations from } \\
\text { the definition of } \\
\text { the numerator }\end{array}$ & $\begin{array}{l}\text { Additional } \\
\text { years } \\
\text { available }\end{array}$ & Comments \\
\hline Latvia & $\begin{array}{l}\text { Register of the Patients of } \\
\text { Diabetes Mellitus }\end{array}$ & & $\begin{array}{l}\text { All patients of } \\
\text { diabetes (type I } \\
\text { and type II) in the } \\
\text { age } 18 \text { and over, } \\
\text { who are include } \\
\text { in the Diabetic } \\
\text { Register. It } \\
\text { includes patients } \\
\text { who are in } \\
\text { primary care and } \\
\text { those in hospitals } \\
\text { who are } \\
\text { registered by } \\
\text { their physician as } \\
\text { a diabetic in the } \\
\text { register. }\end{array}$ & $\begin{array}{l}\text { Number of diabetic } \\
\text { patients with major } \\
\text { (above or below } \\
\text { knee) amputations } \\
\text { in given year. The } \\
\text { number isn't very } \\
\text { correct, because } \\
\text { lower extremity } \\
\text { amputations are } \\
\text { defined as "yes" or } \\
\text { "no" in Diabetic } \\
\text { Register Card } \\
\text { (procedure code is } \\
\text { not included, and } \\
\text { some amputations } \\
\text { maybe weren't } \\
\text { related to diabetes } \\
\text { mellitus. }\end{array}$ & & $\begin{array}{l}\text { Diabetic Register does not cover all population of diabetics, } \\
\text { because there are unregistered cases (problem with data } \\
\text { collection from Family doctors). }\end{array}$ \\
\hline
\end{tabular}


Table 26. Major amputation in diabetics, comparability issues

\begin{tabular}{|c|c|c|c|}
\hline & & \multicolumn{2}{|c|}{ Comparability Implications } \\
\hline & & Minor & Severe \\
\hline 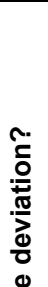 & Possible & $\begin{array}{l}\text { 1. Data available for different years } \\
\text { 2. Data available for slightly different } \\
\text { age ranges } \\
\text { 3. Different procedure codes } \\
\text { included. Unclear how comparable } \\
\text { they are between countries. }\end{array}$ & $\begin{array}{l}\text { 1. Different diagnostic codes } \\
\text { used to capture diabetic } \\
\text { population in hospital } \\
\text { discharge data. }\end{array}$ \\
\hline 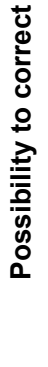 & Unlikely & & $\begin{array}{l}\text { 1. For the denominator, } \\
\text { population based surveys and } \\
\text { data obtained from clinical } \\
\text { surveys or records may not be } \\
\text { fully comparable. } \\
\text { 2. Some countries indicated } \\
\text { that the administrative records } \\
\text { may underreport diabetes } \\
\text { because of incomplete } \\
\text { records. }\end{array}$ \\
\hline
\end{tabular}

Possible solutions:

- Footnotes can indicate the year and age deviations

- Footnote (or drop if serious) if there are concerns that administrative data underreport diabetes.

- Data collected with the denominator from population surveys should be separate from those obtained from clinical surveys or records.

- OECD will need to work with countries to ensure that comparable procedures are used to calculate this indicator. 


\section{Post-operative hip fracture rate}

NOTE that this indicator was already tested in (2003-2005) HCQI data collection and it is not yet considered suitable for international comparisons by the HCQI Expert Group. It is included in this paper to illustrate current data concerns with the indicator and possible future solutions.

\section{Operational Definition}

Source: Agency for Healthcare Research and Quality Patient Safety Indicators (AHRQ PSIs).

Numerator: Patients experiencing an in-hospital hip fracture OR fall as defined by the CSP: secondary diagnosis only and excluding patients with trauma or metastatic cancer as any diagnosis; excluding patients with principal diagnosis of seizure, syncope, stroke, coma, cardiac arrest, or poisoning; excluding patients in MDC 8.

Denominator: Inpatients undergoing major surgery OR minor or miscellaneous surgery OR invasive cardiac procedures OR invasive radiologic procedures OR endoscopy OR medical patients OR all patients as defined by the CSP.

\section{Importance}

93. Clinical significance: Falls are a leading cause of adverse event in acute care hospitals. Up to $20 \%$ or 1 in 5 elderly people fall during recovery from illness (many patients are "at risk" because of problematic medication effect, rehabilitation, etc). Falls are associated with functional disability and injury, increased length of stay, and risk of nursing home placement from hospital. Patient falls are also a significant liability issue for hospital risk-management, because many falls and their damaging consequences are preventable. Falls may be caused by the persons' health status, response to medication or anaesthesia, external factors (wet floor, etc.) or other factors. Reducing risk of falls is an important quality of care issue for hospitals (Iezzoni, 1994).

94. The incidence of hip fracture is related with demographic factors (and others) such as: age, gender, racial difference, rural vs. urban, institutional vs. community dwelling and family history. Two thirds of all hip fractures occur among women. Hip fracture incidence rate from different countries within Europe appear to vary substantially with the highest incidences found in Northern Europe and the lowest in Mediterranean area. Highest rates are found in white populations and lower rates are found in Asian and developing countries. Rural population have lower incidence than urban population. Institutionalised elderly people also have higher rates.

95. Policy importance: Prevention of falls is an important factor in hospital management. It's an important aspect for patients, hospital managers, and visitors. Failure to provide safe conditions in hospital, and a safe environment can lead to falls, which may result in injuries. These injuries may lead to complications and decrease in mobility. In other hand, falls may have impact in patient's perception of safety and psychological well-being.

\section{Scientific Soundness}

96. Evidence supporting indicator validity: The review of this indicator by the AHRQ study group constructing the AHRQ PSIs found post-operative hip fracture generally performs well on several different dimensions, including reliability, bias, relatedness of indicators, and persistence over time (AHRQ, 2005). 
A study conducted a study in Canadian province of Saskatchewan from 1983 through 1985. They found six factors independently associated with a significant increased risk of in-hospital hip fracture: impaired vision; unassisted ambulation, confusion, psychotropic drug use, lowest height tercile and prior in-hospital fall (Lichenstein, 1994). Another study from Canada noted the preventability of falls in the hospital setting (O'Connor, 2006). The American Nurses Association, its state associations, and the California Nursing Outcomes Coalition have identified the number of patient falls leading to injury per 1,000 patient days (based on clinical data collection) as a "nursing-sensitive quality indicator for acute care settings"(McDonald, 2002).

\section{Feasibility}

97. Data availability: Postoperative hip-fractures or fall rates are available for 13 countries (Table 27). The data were reported for years ranging from 2002 to 2005. The OECD definition is for all ages, and five countries had deviations in the age range. All countries use hospital records for the numerator and denominator, but the exclusion criteria of OECD definition were not apply in most of the countries and the codes included varied widely across reporting countries. Several countries expressed their concern about the underestimation of these events due to the scarce codification of patient safety issues. Thus hospital records may be incomplete. All this is of serious concern to comparability.

98. Comparability issues: Detailed documentation and assessment is provided in Table 28. There are serious concerns about comparability both from the estimation of the denominator, and the numerator, the HCQI project will have to ensure that countries apply the same inclusion and exclusion criteria in their calculations. Also some countries rely on ICD9 for codification while others do on ICD10, the impact of this choice in the rates yielded needs to be evaluated by the Secretariat.

99. Overall assessment: thirteen countries provided data on this indicator. While information for this indicator might exist in hospital records of other countries as well, it is unclear how many countries would be able to construct this indicator on a routine basis. Additionally, a significant amount of analytic work will have to be done in order to ensure that the data are internationally comparable. However, data comparability and availability for this indicator is likely to improve due to the work of the Patient Safety Expert Subgroup and the indicator may warrant examination in the future as part of HCQI indicator updates.

Table 27. Post-operative hip fracture rate per 100 discharges

\begin{tabular}{|l|c|c|}
\hline \multicolumn{1}{|c|}{ Country } & Rate per $\mathbf{1 0 0}$ & Data year \\
\hline Australia & 0.77 & $2004-2005$ \\
\hline Canada & 0.07 & $2004-2005$ \\
\hline Denmark & 0.63 & 2005 \\
\hline Finland & 0.28 & 2005 \\
\hline Italy & 0.07 & 2004 \\
\hline Japan & 0.01 & 2005 \\
\hline Mexico & 0.08 & 2005 \\
\hline Netherlands & 0.05 & 2004 \\
\hline Norway & 0.57 & 2005 \\
\hline Portugal & 1.76 & 2004 \\
\hline Spain & 0.08 & 2004 \\
\hline Sweden & 0.05 & 2004 \\
\hline United States & 2.60 & 2002 \\
\hline
\end{tabular}


DELSA/HEA/WD/HWP(2007)4

Post-operative hip fracture rate, sources and methods

\begin{tabular}{|c|c|c|c|c|c|c|}
\hline Country & Source & $\begin{array}{c}\text { Age } \\
\text { groups } \\
\text { included }\end{array}$ & $\begin{array}{l}\text { Deviations from the definition of } \\
\text { the denominator }\end{array}$ & $\begin{array}{l}\text { Deviations from the } \\
\text { definition of the numerator }\end{array}$ & $\begin{array}{l}\text { Additional years } \\
\text { available }\end{array}$ & Comments \\
\hline Australia & $\begin{array}{l}\text { AlHW National Hospital } \\
\text { Morbidity Database }\end{array}$ & & $\begin{array}{l}\text { Australian definitions of Medical, } \\
\text { Surgical and Other discharges based } \\
\text { on Australian Refined Diagnosis } \\
\text { Related Groups (AN-DRG) version } \\
5.1 \text { have been used. These may not } \\
\text { match international definitions. }\end{array}$ & & $2004-2005$ & $\begin{array}{l}\text { Discharges as principal diagnosis or any } \\
\text { diagnosis also available. }\end{array}$ \\
\hline \multicolumn{7}{|c|}{ 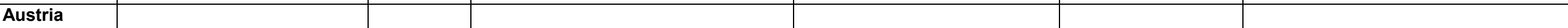 } \\
\hline Canada & $\begin{array}{l}\text { Discharge Abstract } \\
\text { Database }\end{array}$ & & 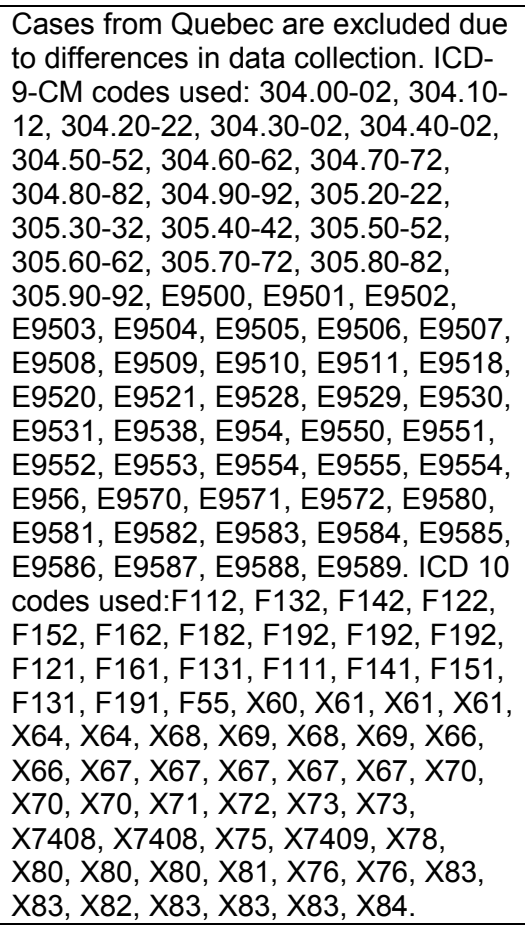 & $\begin{array}{l}\text { We used ICD-10-CA codes } \\
\text { instead of ICD-9-CM. }\end{array}$ & $\begin{array}{l}\text { 1995-1996 to 2004- } \\
2005 .\end{array}$ & \\
\hline
\end{tabular}


DELSA/HEA/WD/HWP(2007)4

\begin{tabular}{|c|c|c|c|c|c|c|}
\hline Country & Source & $\begin{array}{c}\text { Age } \\
\text { groups } \\
\text { included }\end{array}$ & $\begin{array}{l}\text { Deviations from the definition of } \\
\text { the denominator }\end{array}$ & $\begin{array}{l}\text { Deviations from the } \\
\text { definition of the numerator }\end{array}$ & $\begin{array}{l}\text { Additional years } \\
\text { available }\end{array}$ & Comments \\
\hline Denmark & $\begin{array}{l}\text { National hospital discharge } \\
\text { register }\end{array}$ & & & & & $\begin{array}{l}\text { The numbers provided are preliminary and will } \\
\text { change as the register still receives data from } \\
\text { the hospitals. }\end{array}$ \\
\hline Finland & Hospital Discharge Register & & & & & \\
\hline Italy & $\begin{array}{l}\text { Ministry of Health - National } \\
\text { discharges database }\end{array}$ & & $\begin{array}{l}\text { All surgical discharges }(18+) \text { without } \\
\text { any exclusion. }\end{array}$ & & $2001+$ & $\begin{array}{l}\text { Numerator: count of different patients with code } \\
\text { for hip fracture in any secondary diagnosis } \\
\text { field. Value: numerator/denominator }{ }^{\star} 100 \text {. }\end{array}$ \\
\hline Japan & $\begin{array}{l}\text { Japan Medical Data Center, } \\
\text { Inc }\end{array}$ & All & & & & \\
\hline Mexico & $\begin{array}{l}\text { SIAIS.- Sistema de } \\
\text { Información de Atención } \\
\text { Integral a la Salud IMSS } \\
\text { (DTIES) División Técnica de } \\
\text { Información Estadistica en } \\
\text { Salud }\end{array}$ & $20+$ & $\begin{array}{l}20 \text { years and older and it refers to ICD } \\
10 \text { Codes equivalent (unofficial ICD-9, } \\
\text { ICD-10 codes }\end{array}$ & $\begin{array}{l}20 \text { years and older and to ICD } \\
10 \text { Codes } 5720-22\end{array}$ & & Nationally representative \\
\hline Netherlands & $\begin{array}{l}\text { National Medical Registry, } \\
\text { owned by PRISMANT. } \\
\text { Calculations and methods by } \\
\text { Statistics Netherlands in } \\
\text { cooperation with Institute for } \\
\text { Public Health and the } \\
\text { Environment }\end{array}$ & $18+$ & $\begin{array}{l}\text { All discharges with surgery excluding } \\
\text { discharges with the following reasons } \\
\text { for admission: poisoning due to } \\
\text { anaesthetics, drug dependence, drug } \\
\text { abuse or self-inflected injury. } \\
\text { Following ICD-9-CM codes: } 304, \\
\text { 305.2-305.9, 968.1-4, 968.7, E855.1, } \\
\text { E950-E958. }\end{array}$ & & & $\begin{array}{l}\text { In calculating the rate of post-operative hip } \\
\text { fracture, three selection steps were made: } 1 \text {. } \\
\text { selection of discharges with surgery (Dutch } \\
\text { Classification of Medical Specialist CMSV, } \\
\text { version 2.6: } 5011-5719,5738-5999,8724-\text {, } \\
8851,9277 \text {. Surgery of the hip CMSV 2.6 } \\
\text { codes: } 5789.54,5790.06,5790.13,5790.24, \\
5792.06,5792.16,5792.23,5792.33,5792.34 \text {, } \\
5792.6,5815,5816.2 \text {. Exclusion of discharges } \\
\text { with drug dependence, drug abuse or self- } \\
\text { inflected injury as primary reason for } \\
\text { admission. } 3 \text {. Calculating all discharges with a } \\
\text { hip fracture as secondary diagnosis. Hip } \\
\text { fractures as a secondary diagnosis after hip } \\
\text { surgery, were included. Includes clinical } \\
\text { admissions (with one overnight stay or more) } \\
\text { and admissions without an overnight stay (=day } \\
\text { care). Discharges from small specialised } \\
\text { hospitals are excluded. }\end{array}$ \\
\hline
\end{tabular}


DELSA/HEA/WD/HWP(2007)4

\begin{tabular}{|c|c|c|c|c|c|c|}
\hline Country & Source & $\begin{array}{c}\text { Age } \\
\text { groups } \\
\text { included }\end{array}$ & $\begin{array}{l}\text { Deviations from the definition of } \\
\text { the denominator }\end{array}$ & $\begin{array}{l}\text { Deviations from the } \\
\text { definition of the numerator }\end{array}$ & $\begin{array}{l}\text { Additional years } \\
\text { available }\end{array}$ & Comments \\
\hline Norway & Norwegian Patientregister & & & & $2001+$ & $\begin{array}{l}\text { Numerator: ICD-10 codes which are } \\
\text { comparable with the given ICD-9 codes are } \\
\text { used. Diagnoses: S72.0, S72.1, S72.2. } \\
\text { Norwegian Patientregister has no unique } \\
\text { patient identifier. This means that the same } \\
\text { person may appear in the statistics several } \\
\text { times during the year if this person gets } \\
\text { treatment in different hospitals during the year. }\end{array}$ \\
\hline Portugal & DRGs & & & & 1993-2004 & $\begin{array}{l}\text { Numerator: Discharges with ICD-9-CM for hip } \\
\text { fracture in any diagnosis field; denominator: all } \\
\text { surgical discharges } 81.53 \text { age } 18 \text { and over }\end{array}$ \\
\hline Spain & $\begin{array}{l}\text { Hospital Discharge Minimum } \\
\text { Data Set (Conjunto Mínimo } \\
\text { Básico de Datos CMBD). } \\
\text { Ministerio de Sanidad y } \\
\text { Consumo. Instituto de } \\
\text { Información Sanitaria. } \\
\text { Registro de altas } 2004\end{array}$ & & & $\begin{array}{l}\text { Only discharge age }>18 \text { and } \\
\text { surgical DRG (no exclusions }\end{array}$ & 1997 & $\begin{array}{l}\text { No indications about the hip fracture being } \\
\text { suffered after o earlier than the main surgical } \\
\text { procedure. }\end{array}$ \\
\hline $\begin{array}{l}\text { United } \\
\text { States }\end{array}$ & $\begin{array}{l}\text { Agency for Healthcare } \\
\text { Research and Quality } \\
\text { (AHRQ) Patient Safety } \\
\text { Indicators (PSI) }\end{array}$ & & $\begin{array}{l}\text { Inpatient hospital surgical discharges } \\
\text { age } 18 \text { and over who were not } \\
\text { susceptible to falling, excluding } \\
\text { patients with diseases and disorder of } \\
\text { musculoskeletal system and } \\
\text { connective tissue; patients admitted } \\
\text { for seizures, syncope, stroke, coma, } \\
\text { cardiac arrest, poisoning, trauma, } \\
\text { delirium, psychoses, or anoxic brain } \\
\text { injury; patients with metastatic cancer, } \\
\text { lymphoid malignancy, bone } \\
\text { malignancy, or self-inflicted injury; and } \\
\text { obstetrical patients. }\end{array}$ & $\begin{array}{l}\text { Subset of the denominator with } \\
\text { any secondary diagnosis } \\
\text { indicating hip fracture (ICD-9- } \\
\text { CM code 8200-8203, 8208, } \\
8209 \text { ). }\end{array}$ & & $\begin{array}{l}\text { Rates are adjusted by age, gender, age-gender } \\
\text { interactions, comorbidities, and Diagnosis } \\
\text { Related Groups (DRG) clusters. When } \\
\text { reporting is by age, the adjustment is by } \\
\text { gender, comorbidities, and DRG clusters; when } \\
\text { reporting is by gender, the adjustment is by } \\
\text { age, comorbidities, and DRG clusters. Although } \\
\text { not all States participate in the HCUP } \\
\text { database, the Nationwide Inpatient Sample is } \\
\text { weighted to give national estimates using } \\
\text { weights based on all U.S. community, non- } \\
\text { rehabilitation hospitals in the American Hospital } \\
\text { Association Annual Survey of Hospitals. }\end{array}$ \\
\hline
\end{tabular}


Table 28. Post-operative hip fracture rate, comparability issues

\begin{tabular}{|c|c|c|c|}
\hline & & \multicolumn{2}{|c|}{ Comparability Implications } \\
\hline & & Minor & Severe \\
\hline 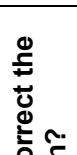 & Possible & $\begin{array}{l}\text { 1. Data available for different years } \\
\text { 2. Different procedure codes included. Unclear } \\
\text { how comparable they are between countries. }\end{array}$ & $\begin{array}{l}\text { 1. Different diagnostic codes and code } \\
\text { systems used across countries (ICD-9 } \\
\text { versus ICD-10). }\end{array}$ \\
\hline 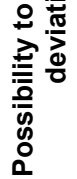 & Unlikely & & $\begin{array}{l}\text { 1. Some countries indicated that empirical } \\
\text { testing of this indicator has indicated the } \\
\text { administrative records may underreport } \\
\text { safety issues because of incomplete } \\
\text { records. }\end{array}$ \\
\hline
\end{tabular}

Possible solutions:

- Footnotes can indicate the year and age deviations

- Footnote (or drop if serious) if there are concerns that administrative data underreport particular safety issue.

- $\quad$ OECD will need to work with countries to ensure that comparable procedures are used to calculate this indicator. 
DELSA/HEA/WD/HWP(2007)4

\section{Transfusion reaction rate}

NOTE that this indicator is new; it has been incorporated for the first time in the 2006 HCQI data collection. It is included in this paper to illustrate current data concerns with the indicator and possible future solutions.

\section{Operational Definition}

Source: AHRQ/CIHI Safety Indicators.

Numerator: Discharges with ICD-9-CM codes for transfusion reaction in any secondary diagnosis field per 100 discharges.

Denominator: All medical and surgical discharges, 18 years and older or MDC 14 (pregnancy, childbirth or puerperium).

\section{Importance}

100. Clinical significance: Transfusion of wrong blood type to the wrong person may have serious effects. The risk of adverse outcome from erroneous transfusion rivals or exceeds current estimates of the risk of acquiring infectious disease by transfusion (Linden, 2000). According the same authors the systems must be redesigned to allow minor fluctuations in human performance, especially in routine tasks. The use of systems designed to prevent specific errors may be helpful (such as convenient access to standard operating procedures instructions in work areas, a blood component lock system that will not allow the access of a component unless there is patient wristband and blood component match, etc.).

101. Recent studies on human error in medicine followed methods derived from the experience gained while analysing large-scale technological disasters (Eagle, 1992; Reason, 1990). They recognised that medical, like technological, accidents nearly always require the conjunction of two types of failures: active failures, mistakes happening while performing a task, and latent failures, or management system errors. The latter ones are more difficult to perceive, because they constitute silent failures residing in a system until human error allows the expression resulting in a major accident (Baele, 1994). According to the author the detection and the correction of the latter type failure, ideally before the occurrence of accidents, is more efficient in improving the overall quality of a system than any action aiming at only active failures. Clinician panellists from AHRQ consider that this indicator very likely reflects actual medical errors. As expected, this indicator proved to be very rare with less than 1 per 10000 cases at risk.

\section{Scientific Soundness}

103. Evidence supporting indicator validity: This indicator was originally proposed by Iezzoni et al.(1992) as part of the Complications Screening Programme (CSP "sentinel events"), along with gas gangrene, CNS abscess, anoxic brain injury, accidental puncture or laceration, wound dehiscence, and foreign body left in (all of which were omitted from this indicator). It was also included as one component of a broader indicator ("adverse events and iatrogenic complications") in AHRQ's original HCUP Quality Indicators. It was proposed by Miller et al. (2001) in the original "AHRQ PSI Algorithms and Groupings," although their definition also includes minor transfusion reactions (999.8), which were omitted from this indicator. 


\section{Operational Issues}

104. Some countries have been made efforts to quantify the magnitude of the non-infectious risks of transfusions include the voluntary SHOT programme; the New York State Department of Health mandatory reporting programme of transfusion - related incidents, accidents and errors; the French Haemovigilance System; and the Belgium SANGUIS Group (Callum, 2001). However, the data may not be available in countries without similar programmes. Moreover, experience in a number of countries as well as in the US has shown that transfusion reactions are very rare events, even within the patient safety indicator group. A study applying the AHRQ PSIs to the US Veterans Administration hospital data found that the transfusion reactions only occurred at a rate of 0.007 per 1000 discharges, the least frequent event across the PSIs (Rosen, 2005).

\section{Feasibility}

105. Data availability: transfusion reaction rates are available for 10 countries (Table 29). The data were reported for years 2004-2005. Five countries reported deviations from OECD definitions in the calculation of numerator and or denominator. The main issue in many of the countries seems to be the restriction of the codes for the numerator to those corresponding to major severe reactions to transfusion. The age related criteria were also unclear for some countries.

106. Also some countries used different procedure codes. This is of serious concern to comparability.

107. Comparability issues: Detailed documentation and assessment is provided in Table 30. There are serious concerns about comparability, both from the estimation of the denominator and numerator because different codes are being included; the HCQI project will have to ensure that countries are reporting the same type of amputations. Some countries indicated that empirical testing of these indicators has indicated the administrative records may underreport safety issues because of incomplete records

108. Overall assessment: Ten countries provided data on this indicator. While information for this indicator might exist in hospital records of other countries as well, it is unclear how many countries would be able to construct this indicator on a routine basis. Additionally, a significant amount of analytic work will have to be done in order to ensure that the data are internationally comparable. However, data comparability and availability for this indicator is likely to improve due to the work of the Patient Safety Expert Subgroup and the indicator may warrant examination in the future as part of HCQI indicator updates.

Table 29. Transfusion reaction rate per 100000 discharges

\begin{tabular}{|l|c|c|}
\hline Country & $\begin{array}{c}\text { Rate per } \\
\mathbf{1 0 0 ~ 0 0 0}\end{array}$ & Data year \\
\hline Australia & 0.31 & $2004-2005$ \\
\hline Canada & 1.09 & $2004-2005$ \\
\hline Czech Republic & 0.08 & 2005 \\
\hline Denmark & 0.58 & 2004 \\
\hline Finland & 0.08 & 2004 \\
\hline Italy & 1.05 & 2004 \\
\hline Netherlands & 0.21 & 2005 \\
\hline Portugal & 19.30 & 2004 \\
\hline Spain & 0.61 & 2004 \\
\hline Sweden & 29.40 & 2004 \\
\hline
\end{tabular}


DELSA/HEA/WD/HWP(2007)4

Transfusion reaction rate, sources and methods

\begin{tabular}{|c|c|c|c|c|c|}
\hline Country & Source & $\begin{array}{l}\text { Deviations from the definition } \\
\text { of the denominator }\end{array}$ & $\begin{array}{l}\text { Deviations from the } \\
\text { definition of the numerator }\end{array}$ & $\begin{array}{l}\text { Additiona } \\
\text { I years } \\
\text { available }\end{array}$ & Comments \\
\hline Australia & $\begin{array}{l}\text { AlHW National Hospital Morbidity } \\
\text { Database }\end{array}$ & $\begin{array}{l}\text { Australian definitions of Medical, } \\
\text { Surgical and other discharges } \\
\text { based on Australian Refined } \\
\text { Diagnosis Related Groups (AN- } \\
\text { DRG) version } 5.1 \text { have been } \\
\text { used. These may not match } \\
\text { international definitions. }\end{array}$ & & $\begin{array}{l}2004- \\
2005\end{array}$ & $\begin{array}{l}\text { Discharges as principal diagnosis or any diagnosis } \\
\text { also available. }\end{array}$ \\
\hline Canada & Discharge Abstract Database & $\begin{array}{l}\text { Cases from Quebec are } \\
\text { excluded due to differences in } \\
\text { data collection }\end{array}$ & & $\begin{array}{l}1995- \\
1996 \text { to } \\
2004- \\
2005\end{array}$ & $\begin{array}{l}\text { The selection of surgical/medical/MDC14 was based } \\
\text { on the CMG grouping }\end{array}$ \\
\hline $\begin{array}{l}\text { Czech } \\
\text { Republic }\end{array}$ & $\begin{array}{l}\text { UZIS CR (Annual Report on the } \\
\text { activity of Transfusion Services) }\end{array}$ & $\begin{array}{l}\text { Total number of hospitalised } \\
\text { persons }\end{array}$ & $\begin{array}{l}\text { Includes just number of } \\
\text { serious adverse reactions - } \\
\text { immunological haemolysis } \\
\text { due to AB0 incompatibility, } \\
\text { immunological haemolysis } \\
\text { due to other allo-antibody. }\end{array}$ & & $\begin{array}{l}\text { Our Annual Reporting System was conformed to the } \\
\text { European Commission Directives: } 2005 / 61 / E C \text { and } \\
2002 / 98 / E C \text { concerning setting standards of quality } \\
\text { and safety for the collecting, testing, processing, } \\
\text { storage and distribution of human blood components. } \\
\text { No other data concerning transfusion reactions are } \\
\text { collected. }\end{array}$ \\
\hline Denmark & National Hospital Discharge Register & & & & $\begin{array}{l}\text { Data is for the year 2004. The denominator does not } \\
\text { include MDC } 14\end{array}$ \\
\hline Finland & Hospital Discharge Register & & $\begin{array}{l}\text { Numerator: also age } 18 \text { and } \\
\text { older. There was } 1 \text { case in } \\
123203 .\end{array}$ & & \\
\hline Italy & $\begin{array}{l}\text { Ministry of Health - National } \\
\text { database of discharges }\end{array}$ & $\begin{array}{l}\text { All surgical and medical } \\
\text { discharges, } 18 \text { years and older }\end{array}$ & $\begin{array}{l}\text { Only ICD } 9 \text { CM } 999.6 \text { or } 999.7 \\
\text { in any secondary diagnosis } \\
\text { field }\end{array}$ & $2001+$ & \\
\hline Netherlands & $\begin{array}{l}\text { National Medical Registry, owned by } \\
\text { PRISMANT. Calculations and } \\
\text { methods by Statistics Netherlands in } \\
\text { cooperation with Institute for Public } \\
\text { Health and the Environment }\end{array}$ & $\begin{array}{l}\text { Consists of all surgical and } \\
\text { medical discharges }(18+\text { or } \\
\text { MDC 14)'. We assume OECD } \\
\text { means all hospital admissions } \\
\text { for persons } 18 \text { years or older } \\
\text { and hospital admission among } \\
\text { adolescents for MDC } 14 \text {. We }\end{array}$ & $\begin{array}{l}\text { It is unclear whether } \\
\text { newborns and children have } \\
\text { to be included. Nevertheless, } \\
\text { we decided to present only } \\
\text { data for adults }(18+) \text { and girls } \\
\text { of } 10 \text { years old or older. }\end{array}$ & & $\begin{array}{l}\text { From the National Medical Registry we include all } \\
\text { discharges with a transfusion reaction as primary or } \\
\text { secondary admission diagnosis. In all rates, clinical } \\
\text { admissions (with one overnight stay or more) and } \\
\text { admissions without an overnight stay (=day care) were } \\
\text { included. Discharges from small specialised hospitals } \\
\text { are excluded, for example eye clinics, clinics for }\end{array}$ \\
\hline
\end{tabular}


DELSA/HEA/WD/HWP(2007)4

\begin{tabular}{|c|c|c|c|c|c|}
\hline Country & Source & $\begin{array}{l}\text { Deviations from the definition } \\
\text { of the denominator }\end{array}$ & $\begin{array}{l}\text { Deviations from the } \\
\text { definition of the numerator }\end{array}$ & $\begin{array}{l}\text { Additiona } \\
\text { I years } \\
\text { available }\end{array}$ & Comments \\
\hline & & $\begin{array}{l}\text { expected as denominator the } \\
\text { number of transfusions, number } \\
\text { of blood units administered or } \\
\text { the number of patients with a } \\
\text { transfusion. Major Diagnostic } \\
\text { Categories (MDCs) are not } \\
\text { used in all countries, at least not } \\
\text { in the Netherlands. We assume } \\
\text { that MDC } 14 \text { refers to health } \\
\text { status described in Chapter } 11 \\
\text { of ICD-9 (codes 630-676) and } \\
\text { several V-codes (V22-V24, V27, } \\
\text { V28). The denominator is only } \\
\text { defined for persons } 18 \text { years or } \\
\text { older and women admitted for } \\
\text { problems occurring during } \\
\text { pregnancy, childbirth and } \\
\text { puerperium. }\end{array}$ & & & epilepsy, asthma clinics, clinics for rehabilitation \\
\hline Portugal & DGRs & & & $\begin{array}{l}1993- \\
2004\end{array}$ & $\begin{array}{l}\text { The denominator represents the number of blood } \\
\text { transfusions }\end{array}$ \\
\hline $\begin{array}{l}\text { Slovak } \\
\text { Republic }\end{array}$ & & & & & $\begin{array}{l}\text { Alternative available source could be the record of } \\
\text { patient complaints handled at the Bureau for } \\
\text { Supervision of Health Care. }\end{array}$ \\
\hline Spain & $\begin{array}{l}\text { Hospital Discharge Minimum Data } \\
\text { Set (Conjunto Mínimo Básico de } \\
\text { Datos CMBD). Ministerio de Sanidad } \\
\text { y Consumo. Instituto de Información } \\
\text { Sanitaria. Registro de altas 2004. }\end{array}$ & & $\begin{array}{l}\text { Selected age also (same } \\
\text { criteria than denominator) }\end{array}$ & $\begin{array}{l}\text { From } \\
1997\end{array}$ & $\begin{array}{l}\text { The term "reaction" also includes codes (ICD9) related } \\
\text { to other type of reactions, apart from incompatibility or } \\
\text { mismatched blood. }\end{array}$ \\
\hline
\end{tabular}


Table 30. Transfusion reaction rate, comparability issues

\begin{tabular}{|c|c|c|c|}
\hline & & \multicolumn{2}{|c|}{ Comparability Implications } \\
\hline & & Minor & Severe \\
\hline \multirow{2}{*}{ 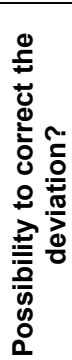 } & Possible & $\begin{array}{l}\text { 1. Different ages included } \\
\text { 2. Different procedure codes included. Unclear } \\
\text { how comparable they are between countries. }\end{array}$ & $\begin{array}{l}\text { 1. Different diagnostic codes and code } \\
\text { systems used across countries (ICD-9 } \\
\text { versus ICD-10). }\end{array}$ \\
\hline & Unlikely & & $\begin{array}{l}\text { 1. Some countries indicated that } \\
\text { empirical testing of these indicators } \\
\text { has indicated the administrative } \\
\text { records may underreport safety issues } \\
\text { because of incomplete records. }\end{array}$ \\
\hline
\end{tabular}

Possible solutions:

- Footnotes can indicate the year and age deviations

- Footnote (or drop if serious) if there are concerns that administrative data underreport particular safety issue.

- OECD will need to work with countries to ensure that comparable procedures are used to calculate this indicator. 


\section{Uncontrolled diabetes admission rate}

NOTE that this indicator is new and has been incorporated for the first time in 2006 HCQI data collection. It is included in this paper to illustrate current data concerns with the indicator and possible future solutions.

\section{Operational Definition}

Source: AHRQ Prevention Quality Indicators.

Numerator: All non-maternal discharges of age 18 years and older with ICD-9-CM principal diagnosis code for uncontrolled diabetes, without mention of a short-term or long-term complication.

Denominator: Total population.

\section{Importance}

109. Clinical significance of process or outcome: Avoidable hospitalisations are those conditions that could have been avoided if proper ambulatory care had been received and can thus be seen as a measure of access to appropriate medical care. While not all admissions for ambulatory care sensitive conditions are avoidable, it is assumed that appropriate prior ambulatory care could prevent the onset of this type of illness or condition, control an acute episodic illness or condition, or manage a chronic disease or condition. A disproportionately high rate is presumed to reflect problems in obtaining access to primary care (Weissman, 1992). In terms of avoiding uncontrolled diabetes admission rates, in the US, the Healthy People 2010 effort has set a national goal to reduce the rate of admissions for uncontrolled diabetes for persons 18-64 years of age from 7.2 per 10000 to 5.4 per 10000 population (HHS, 2000). Studies in the US have shown similar rates of admissions for uncontrolled diabetes for men and women and across racial and ethnic groups, unlike some other preventable hospitalisation indicators for diabetes care (Correa-deAraujo, 2006).

110. Identification of process/outcome as quality problem: The rate of Ambulatory Care Sensitive (ACS) hospitalisations is considered an index of access of a population to adequate primary care. These are hospitalisations for selected diagnoses some of which might reasonably have been prevented if primary care had been received in time. ACS hospitalisations are elevated in low-income areas and in rural/frontier areas.

111. Policy importance: Given the high cost of hospital care and the high prevalence of the disease included in this indicator, elevated ACS hospitalisation rates could point not only towards possibilities to improve quality but also to substantial cost savings, if better primary care were provided. In addition, the ACS hospitalisation rate appears sensitive to the presence or absence of economic barriers to access. It has been reported to be lower and/or less correlated with socioeconomic status in countries with national health insurance (Billings, 1996).

112. Susceptibility to being influenced by the health system: Appropriate prior ambulatory care could prevent the onset of an illness or condition; control an acute episodic illness or condition; or manage a chronic disease or condition (Anderson, 1996). 


\section{DELSA/HEA/WD/HWP(2007)4}

\section{Scientific Soundness}

113. Face validity: Managing chronic diseases to prevent complications and exacerbations is regarded as a core function of the primary health care system. Good quality outpatient care of diabetes has been shown to lead to reductions in a range of diabetes admissions.

114. Content validity: As mentioned above, several groups have advocated measures of ACS hospitalisation rates. The fact that hospital admission diagnoses are readily available in most countries implies that the indicator can be easily constructed. However, it should be mentioned that there remains some controversy about this (and similar) measures as a quality indicator, because ACS hospitalisation rates reflect access to, as well as quality of, primary care. Also, defining the appropriate level of hospital admission rates for those conditions is difficult, because in a subset of cases an admission is clearly warranted. Based on scientific studies, this indicator is only moderately precise with area level rate of 34.7 per 100,000 (based on US estimates) and a standard deviation of 28.1. ${ }^{21}$

\section{Feasibility}

115. Data availability: Uncontrolled diabetes admission rates are available for 13 countries (Table 31 ). The data were reported for years 2004-2005. Eight countries reported deviations from OECD definitions in the calculation of numerator. All countries use hospital records for the numerator, but the diagnostic codes to capture uncontrolled diabetes admission varied. Countries indicated that hospital records may be incompletely coded, and may underestimate the number of admissions.

116. Comparability issues: Detailed documentation and assessment is provided in Table 32. There are serious concerns about comparability from the estimation of the numerator; the HCQI project will have to ensure that countries are reporting the same type of admissions.

117. Overall assessment: Thirteen countries provided data on this indicator. While information for this indicator might exist in hospital records of other countries as well, it is unclear how many countries would be able to construct this indicator on a routine basis. Additionally, a significant amount of analytic work will have to be done in order to ensure that the data are internationally comparable. However, data comparability and availability for this indicator is likely to improve due to the work of the Primary Care and Prevention Expert Subgroup and the indicator may warrant examination in the future as part of HCQI indicator updates.

Table 31. Uncontrolled diabetes admission rate

\begin{tabular}{|l|c|c|}
\hline \multicolumn{1}{|c|}{ Country } & $\begin{array}{c}\text { Rate per } \\
\mathbf{1 0 0 ~ 0 0 0}\end{array}$ & Data year \\
\hline Australia & 18.9 & $2004-2005$ \\
\hline Austria & 34.2 & 2004 \\
\hline Canada & 22.1 & $2004-2005$ \\
\hline Czech Republic & 364.6 & 2004 \\
\hline Finland & 12.7 & 2005 \\
\hline Italy & 70.9 & 2004 \\
\hline Japan & 294.2 & 2005 \\
\hline Mexico & 7.6 & 2005 \\
\hline Norway & 90.0 & 2005 \\
\hline Portugal & 23.3 & 2004 \\
\hline Spain & 79.9 & 2004 \\
\hline Sweden & 71.9 & 2004 \\
\hline United States & 25.4 & NA \\
\hline
\end{tabular}

Notes: NA stands for no data available.

${ }^{27}$ Agency for Healthcare Research and Quality. Guide to the Prevention Quality Indicators. (Rockville, MD: February 2006.) Version 3.0a. 
DELSA/HEA/WD/HWP(2007)4

Uncontrolled diabetes admission rate, sources and methods

\begin{tabular}{|c|c|c|c|c|c|c|}
\hline Country & Source & $\begin{array}{l}\text { Age } \\
\text { groups } \\
\text { included }\end{array}$ & $\begin{array}{l}\text { Deviations from the definition of the } \\
\text { denominator }\end{array}$ & $\begin{array}{l}\text { Deviations from the definition of } \\
\text { the numerator }\end{array}$ & $\begin{array}{l}\text { Addition } \\
\text { al years } \\
\text { available }\end{array}$ & Comments \\
\hline Australia & $\begin{array}{l}\text { AlHW National Hospital } \\
\text { Morbidity Database }\end{array}$ & & & $\begin{array}{l}\text { ICD-10-AM E10.1, E11.1, E12.1, } \\
\text { E13.1, E14.1 }\end{array}$ & & \\
\hline Austria & Statistics Austria & $15+$ & & $\begin{array}{l}\text { Principle diagnoses given in ICD-10 } \\
\text { codes: E100, E110, E120, E130, } \\
\text { E140, E101, E111, E121, E131, E141 }\end{array}$ & & $\begin{array}{l}\text { Exclusion of pregnancy, childbirth and } \\
\text { puerperium is not possible. }\end{array}$ \\
\hline Canada & $\begin{array}{l}\text { Discharge Abstract Database, } \\
\mathrm{ClHI}\end{array}$ & & $\begin{array}{l}\text { Quebec population figures are excluded } \\
\text { as the numerator excludes Quebec cases } \\
\text { (see Other Comments below). }\end{array}$ & & & $\begin{array}{l}\text { 1. For FY2004, CIHI Coding Standards state } \\
\text { that a blood glucose level of less than } 14 \\
\text { mmol/L(PC) or } 10 \text { mmol/L (fasting) in the } \\
\text { presence of physician documentation that } \\
\text { the patient was 'uncontrolled' would place } \\
\text { the case in an 'adequately controlled' code } \\
\text { because the blood sugar level was not truly } \\
\text { in the uncontrolled parameters. Therefore, } \\
\text { the number of cases of 'uncontrolled' } \\
\text { diabetes (as per the ICD-9-CM rule of coding } \\
\text { uncontrolled when stated) in Canada is likely } \\
\text { to be lower than in other jurisdictions. } 2 \text {. } \\
\text { Cases were extracted based on a most } \\
\text { responsible diagnosis of the condition of } \\
\text { interest. The most responsible diagnosis is } \\
\text { defined as the one diagnosis or condition } \\
\text { that can be described as being the most } \\
\text { responsible for the patient's stay in hospital. } \\
\text { In the event that multiple diagnoses are } \\
\text { listed, select the most responsible diagnosis } \\
\text { from the condition associated with the } \\
\text { longest length of stay or most resource } \\
\text { intense. . Due to an inability to separate } \\
\text { uncontrolled diabetes cases coded in ICD-9, } \\
\text { data submitted by the province of Quebec } \\
\text { are excluded. }\end{array}$ \\
\hline
\end{tabular}


DELSA/HEA/WD/HWP(2007)4

\begin{tabular}{|c|c|c|c|c|c|c|}
\hline Country & Source & $\begin{array}{l}\text { Age } \\
\text { groups } \\
\text { included }\end{array}$ & $\begin{array}{l}\text { Deviations from the definition of the } \\
\text { denominator }\end{array}$ & $\begin{array}{l}\text { Deviations from the definition of } \\
\text { the numerator }\end{array}$ & $\begin{array}{l}\text { Addition } \\
\text { al years } \\
\text { available }\end{array}$ & Comments \\
\hline $\begin{array}{l}\text { Czech } \\
\text { Republic }\end{array}$ & $\begin{array}{l}\text { National Registry of } \\
\text { Hospitalized Patients }\end{array}$ & $15+$ & Population $20+$ as of 1 st of July & $\begin{array}{l}\text { Includes all hospitalised patients in } \\
\text { general hospitals for dg. of E10-E14 }\end{array}$ & & \\
\hline Finland & Hospital Discharge Register & & & & $2001+$ & $\begin{array}{l}\text { Overall number was } 482 \text { cases. The } \\
\text { reliability of diagnostic notes is unclear, may } \\
\text { be an over or underestimation }\end{array}$ \\
\hline Italy & $\begin{array}{l}\text { Ministry of Health - National } \\
\text { discharges database }\end{array}$ & & & & & \\
\hline Japan & Patient Survey & $20+$ & $20+$ & Includes all diabetes (ICD9 250) & & $\begin{array}{l}\text { According to the Patient Survey, the number } \\
\text { of discharges in September } 2005 \text { over } 20 \\
\text { years with primary diagnosis of diabetes was } \\
\text { estimated as approximately } 25400 \text { (subject } \\
\text { to wide margin of error inherent in sampling } \\
\text { survey). Multiplied by } 12 \text {, the annual number } \\
\text { of discharges is estimated to be } 304800 \text {. } \\
\text { Divided by Japan's population over } 20 \text { as of } \\
\text { October } 2005 \text { [ } 103.56 \text { million], the rate was } \\
\text { calculated as } 24.52 \text { / } 100,000 \text { monthly or } \\
294.24 / 100000 \text { annually. Since the Patient } \\
\text { Survey covers only one month, the } \\
\text { numerator (estimated } 26700 \text { ) was multiplied } \\
\text { by } 12 \text {. }\end{array}$ \\
\hline Mexico & $\begin{array}{l}\text { SIAIS.- Sistema de Información } \\
\text { de Atención Integral a la Salud } \\
\text { IMSS (DTIES) División Técnica } \\
\text { de Información Estadística en } \\
\text { Salud }\end{array}$ & $20+$ & $\begin{array}{l}\text { Only } 20 \text { years and older, exclude } 18 \text { and } \\
19 \text { years old }\end{array}$ & $\begin{array}{l}\text { Work with ICD } 10 \text { Codes (Unofficial } \\
\text { table ICD } 9 \text { and ICD } 10 \text { codes }\end{array}$ & & Nationwide representative \\
\hline Norway & Norwegian Patientregister & & & & & $\begin{array}{l}\text { Numerator: ICD-10 codes which are } \\
\text { comparable with the given ICD-9 codes are } \\
\text { used. Main diagnoses: E10.9, E11.9, E12.9, } \\
\text { E13.9, E14.9. }\end{array}$ \\
\hline Portugal & DRGs & & & & & \\
\hline
\end{tabular}


DELSA/HEA/WD/HWP(2007)4

\begin{tabular}{|c|c|c|c|c|c|c|}
\hline Country & Source & $\begin{array}{c}\text { Age } \\
\text { groups } \\
\text { included }\end{array}$ & $\begin{array}{l}\text { Deviations from the definition of the } \\
\text { denominator }\end{array}$ & $\begin{array}{l}\text { Deviations from the definition of } \\
\text { the numerator }\end{array}$ & $\begin{array}{l}\text { Addition } \\
\text { al years } \\
\text { available }\end{array}$ & Comments \\
\hline $\begin{array}{l}\text { Slovak } \\
\text { Republic }\end{array}$ & & & & & & $\begin{array}{l}\text { Our records do not work with four digit ICD, } \\
\text { therefore it is not possible to consider } \\
\text { exclusions. }\end{array}$ \\
\hline Spain & $\begin{array}{l}\text { Numerator : Hospital Discharge } \\
\text { Minimum Data Set (Conjunto } \\
\text { Mínimo Básico de Datos } \\
\text { CMBD). Ministerio de Sanidad y } \\
\text { Consumo. Instituto de } \\
\text { Información Sanitaria. Registro } \\
\text { de altas } 2004 \text {. Denominator: } \\
\text { Health Interview Survey } 2003\end{array}$ & & & $\begin{array}{l}\text { Number of transferred patients from } \\
\text { other centres not known. }\end{array}$ & & $\begin{array}{l}\text { National patient records (in-patients) do not } \\
\text { include information about transferred } \\
\text { patients from other centres. }\end{array}$ \\
\hline Sweden & $\begin{array}{l}\text { The Swedish Hospital } \\
\text { Discharge Register }\end{array}$ & & & & & \\
\hline $\begin{array}{l}\text { United } \\
\text { States }\end{array}$ & $\begin{array}{l}\text { Agency for Healthcare } \\
\text { Research and Quality (AHRQ) } \\
\text { Prevention Quality Indicators } \\
\text { (PQI) }\end{array}$ & & $\begin{array}{l}\text { U.S. population age } 18 \text { and over. Are } \\
\text { there any deviations from the definition of } \\
\text { the numerator? Discharges age } 18 \text { and } \\
\text { over with principal diagnosis of } \\
\text { uncontrolled diabetes (ICD-9-CM code } \\
250.02,250.03 \text { ) without mention of a } \\
\text { short-term (ketoacidosis, hyperosmolarity, } \\
\text { coma) or long-term complication (renal, } \\
\text { eye, neurological, circulatory, other } \\
\text { unspecified). Obstetric and neonatal } \\
\text { admissions and transfers from other } \\
\text { institutions are excluded. Comments: } \\
\text { Rates are adjusted by age and gender } \\
\text { using the total U.S. population for } 2000 \text { as } \\
\text { the standard population. When reporting } \\
\text { is by age, the adjustment is by gender } \\
\text { only; when reporting is by gender, the } \\
\text { adjustment is by age only. Although not all } \\
\text { States participate in the HCUP database, } \\
\text { the Nationwide Inpatient Sample is } \\
\text { weighted to give national estimates using } \\
\text { weights based on all U.S. community, }\end{array}$ & & & \\
\hline
\end{tabular}


DELSA/HEA/WD/HWP(2007)4

\begin{tabular}{|c|c|c|c|c|c|c|}
\hline Country & Source & $\begin{array}{l}\text { Age } \\
\text { groups } \\
\text { included }\end{array}$ & $\begin{array}{l}\text { Deviations from the definition of the } \\
\text { denominator }\end{array}$ & $\begin{array}{l}\text { Deviations from the definition of } \\
\text { the numerator }\end{array}$ & $\begin{array}{l}\text { Addition } \\
\text { al years } \\
\text { available }\end{array}$ & Comments \\
\hline & & & $\begin{array}{l}\text { non-rehabilitation hospitals in the } \\
\text { American Hospital Association Annual } \\
\text { Survey of Hospitals. }\end{array}$ & & & \\
\hline
\end{tabular}


Table 32. Uncontrolled diabetes admission rate, comparability issues

\begin{tabular}{|c|c|c|c|}
\hline & & \multicolumn{2}{|c|}{ Comparability Implications } \\
\hline & & Minor & Severe \\
\hline$\underset{\substack{c \\
\frac{0}{0}}}{\frac{n}{0}}$ & Possible & $\begin{array}{l}\text { 1. Data available for slightly different age ranges } \\
\text { 2. Different procedure codes included. Unclear } \\
\text { how comparable they are between countries. }\end{array}$ & $\begin{array}{l}\text { 1. Different diagnostic codes used to } \\
\text { capture diabetic population in hospital } \\
\text { discharge data. }\end{array}$ \\
\hline 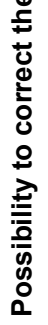 & Unlikely & & $\begin{array}{l}\text { 1. For the denominator, population } \\
\text { based surveys and data obtained from } \\
\text { clinical surveys or records may not be } \\
\text { fully comparable. } \\
\text { 2. Some countries indicated that the } \\
\text { administrative records may } \\
\text { underreport diabetes because of } \\
\text { incomplete records. }\end{array}$ \\
\hline
\end{tabular}

Possible solutions:

- Footnotes can indicate the year and age deviations

- Footnote (or drop if serious) if there are concerns that administrative data underreport diabetes.

- Data collected with the denominator from population surveys should be separate from those obtained from clinical surveys or records.

- OECD will need to work with countries to ensure that comparable procedures are used to calculate this indicator. 
DELSA/HEA/WD/HWP(2007)4

\section{Hypertension admission rate}

NOTE that this indicator is new and has been incorporated for the first time in 2006 HCQI data collection. It is included in this paper to illustrate current data concerns with the indicator and possible future solutions.

\section{Operational Definition}

Source: AHRQ Prevention Quality Indicators.

Numerator: All non-maternal discharges of age 18 years and older with ICD-9-CM principal diagnosis code for hypertension. (Excluding cases: transfer from other institution, MDC 14 (pregnancy, childbirth, and puerperium or with cardiac procedure codes in any field.

Denominator: Total population.

\section{Importance}

118. Clinical significance of process or outcome: Avoidable hospitalisations are those conditions that could have been avoided if proper ambulatory care had been received and can thus be seen as a measure of access to appropriate medical care. While not all admissions for ambulatory care sensitive conditions are avoidable, it is assumed that appropriate prior ambulatory care could prevent the onset of this type of illness or condition, control an acute episodic illness or condition, or manage a chronic disease or condition. A disproportionately high rate is presumed to reflect problems in obtaining access to primary care (Weissman, 1992). While not all admissions for hypertension are inappropriate, it has been shown that good quality primary care can keep hypertensive patients out of the hospital. Moreover, while hypertension is relatively common, hospitalisations for hypertension are relatively rare. One study in the US found that hypertension accounted for only $0.5 \%$ of total admissions for ambulatory care sensitive conditions (Blustein, 1998).

119. Identification of process/outcome as quality problem: The rate of Ambulatory Care Sensitive (ACS) hospitalisations is considered an index of access of a population to adequate primary care. These are hospitalisations for selected diagnoses some of which might reasonably have been prevented if primary care had been received in time. ACS hospitalisations are elevated in low-income areas and in rural/frontier areas. A Italian study on the causes of uncontrolled hypertension concluded that, in addition to patient level factors, the doctor-patient relationship and a good quality of primary care were significant factors in avoiding adverse outcomes (Degli Esposti, 2004).

120. Policy importance: Given the high cost of hospital care and the high prevalence of the disease included in this indicator, elevated ACS hospitalisation rates could point not only towards possibilities to improve quality but also to substantial cost savings, if better primary care were provided. In addition, the ACS hospitalisation rate appears sensitive to the presence or absence of economic barriers to access. It has been reported to be lower and/or less correlated with socioeconomic status in countries with national health insurance (Billings, 1996).

121. Susceptibility to being influenced by the health system: Appropriate prior ambulatory care could prevent the onset of an illness or condition; control an acute episodic illness or condition; or manage a chronic disease or condition (Anderson, 1996). 


\section{Scientific Soundness}

122. Face validity: Managing chronic diseases to prevent complications and exacerbations is regarded as a core task of the primary health care system. Little empirical evidence exists as to the validity of the hypertension avoidable hospitalisations indicator in particular. However, studies have shown that there are significant differences across age and sex, and AHRQ recommends that this indicator be ultimately age and sex adjusted (AHRQ, 2006).

123. Content validity: As mentioned above, several groups have advocated measures of ACS hospitalisation rates. The fact that hospital admission diagnoses are readily available in most countries implies that the indicator can be easily constructed. However, it should be mentioned that there remains some controversy about this (and similar) measures as a quality indicator, because ACS hospitalisation rates reflect access to, as well as quality of, primary care. Also, defining the appropriate level of hospital admission rates for those conditions is difficult, because in a subset of cases an admission is clearly warranted.

\section{Feasibility}

124. Data availability: Uncontrolled diabetes admission rates are available for 15 countries (Table 33). The data were reported for years 2004-2005. Seven countries reported deviations from OECD definitions in the calculation of numerator. All countries use hospital records for the numerator, but the diagnostic codes to capture hypertension admission varied. One country indicated that hospital records may be incompletely coded, and may underestimate the number of admissions.

125. Comparability issues: Detailed documentation and assessment is provided in Table 34. There are serious concerns about comparability from the estimation of the numerator; the HCQI project will have to ensure that countries are reporting the same type of admissions. Another threat for the comparability comes from the variation of hypertension prevalence across countries. There should be explored the need for agestandardisation and the use of a truncated population as a proxy for disease specific population.

126. Overall assessment: Fifteen countries provided data on this indicator. While information for this indicator might exist in hospital records of other countries as well, it is unclear how many countries would be able to construct this indicator on a routine basis. Additionally, a significant amount of analytic work will have to be done in order to ensure that the data are internationally comparable. However, data comparability and availability for this indicator is likely to improve due to the work of the Primary Care and Prevention Expert Subgroup and the indicator may warrant examination in the future as part of HCQI indicator updates. 
Table 33. Hypertension admission rate per 100000 discharges

\begin{tabular}{|l|c|c|}
\hline \multicolumn{1}{|c|}{ Country } & $\begin{array}{c}\text { Rate per } \\
\mathbf{1 0 0 ~ 0 0 0}\end{array}$ & Data year \\
\hline Australia & 43.7 & $2004-2005$ \\
\hline Austria & 474.8 & 2004 \\
\hline Canada & 26.3 & $2004-2005$ \\
\hline Czech Republic & 273.0 & 2004 \\
\hline Denmark & 2.9 & 2005 \\
\hline Finland & 178.1 & 2005 \\
\hline Italy & 174.8 & 2004 \\
\hline Japan & 82.3 & 2005 \\
\hline Mexico & 87.9 & 2005 \\
\hline Netherlands & 35.9 & 2004 \\
\hline Norway & 120.0 & 2005 \\
\hline Portugal & 53.1 & 2004 \\
\hline Spain & 10.4 & 2004 \\
\hline Sweden & 63.8 & 2004 \\
\hline
\end{tabular}

\begin{tabular}{|c|c|c|}
\hline \multicolumn{3}{|c|}{ Non-OECD EU countries } \\
\hline Country & $\begin{array}{c}\text { Rate per } \\
\mathbf{1 0 0} \mathbf{0 0 0}\end{array}$ & Data year \\
\hline \multicolumn{3}{|c|}{677.0} \\
\hline
\end{tabular}

Notes:

1. Data from hospital administered statistics 
DELSA/HEA/WD/HWP(2007)4

Hypertension admission rate, sources and methods

\begin{tabular}{|c|c|c|c|c|c|c|}
\hline Country & Source & $\begin{array}{l}\text { Age } \\
\text { groups } \\
\text { included }\end{array}$ & $\begin{array}{l}\text { Deviations from the definition of } \\
\text { the denominator }\end{array}$ & $\begin{array}{l}\text { Deviations from the definition of } \\
\text { the numerator }\end{array}$ & $\begin{array}{l}\text { Additiona } \\
\text { I years } \\
\text { available }\end{array}$ & Comments \\
\hline Australia & $\begin{array}{lll}\text { AlHW } & \text { National } & \text { Hospital } \\
\text { Morbidity } & \text { Database } & \end{array}$ & & & $\begin{array}{l}\text { Data are for ICD-10-AM I10, I11, I12, } \\
\text { I13. }\end{array}$ & & \\
\hline Austria & Statistics Austria & $15+$ & & $\begin{array}{l}\text { Principle diagnoses given in ICD-10 } \\
\text { codes: I10, I110, I119, I120, I129, } \\
\text { I130, I131, I132, I139 }\end{array}$ & & $\begin{array}{l}\text { No exclusions were made, all discharges with } \\
\text { principle diagnosis of the given codes. }\end{array}$ \\
\hline Canada & $\begin{array}{l}\text { Discharge Abstract Database, } \\
\mathrm{ClHI}\end{array}$ & & $\begin{array}{l}\text { Quebec population figures are } \\
\text { excluded as the numerator excludes } \\
\text { Quebec cases (see Other } \\
\text { Comments below). }\end{array}$ & & & $\begin{array}{l}\text { 1. Corresponding ICD-10-CA codes were } \\
\text { used. } 2 \text {. Cases were extracted based on a } \\
\text { most responsible diagnosis of the condition of } \\
\text { interest. The most responsible diagnosis is } \\
\text { defined as the one diagnosis or condition that } \\
\text { can be described as being the most } \\
\text { responsible for the patient's stay in hospital. } \\
\text { In the event that multiple diagnoses are listed, } \\
\text { select the most responsible diagnosis from } \\
\text { the condition associated with the longest } \\
\text { length of stay or most resource intense. } 3 \text {. } \\
\text { Due to an inability in the ICD-9 coding } \\
\text { classification to separate CHF and renal } \\
\text { failure patients who also present with } \\
\text { hypertension, data submitted by the province } \\
\text { of Quebec were excluded (Quebec was the } \\
\text { only province in Canada coding in ICD- } 9 \text { in } \\
2004 / 05) \text {. }\end{array}$ \\
\hline $\begin{array}{l}\text { Czech } \\
\text { Republic }\end{array}$ & $\begin{array}{lll}\text { National } & \text { Registry } & \text { of } \\
\text { Hospitalized Patients }\end{array}$ & $20+$ & Population $20+$ as of 1st of July & $\begin{array}{l}\text { Includes all hospitalised patients in } \\
\text { general hospitals for diagnosis of } 110\end{array}$ & & \\
\hline Denmark & $\begin{array}{ll}\text { National Hospital Discharge } \\
\text { Register }\end{array}$ & & & & & $\begin{array}{l}\text { There have been no exclusions in the } \\
\text { numerator (it is not possible to write in the } \\
\text { numerator box). The numbers provided are } \\
\text { preliminary and will change as the register still } \\
\text { receives data from the hospitals. It should be } \\
\text { kept in mind, when interpreting this indicator }\end{array}$ \\
\hline
\end{tabular}


DELSA/HEA/WD/HWP(2007)4

\begin{tabular}{|c|c|c|c|c|c|c|}
\hline Country & Source & $\begin{array}{l}\text { Age } \\
\text { groups } \\
\text { included }\end{array}$ & $\begin{array}{l}\text { Deviations from the definition of } \\
\text { the denominator }\end{array}$ & $\begin{array}{l}\text { Deviations from the definition of } \\
\text { the numerator }\end{array}$ & $\begin{array}{l}\text { Additiona } \\
\text { I years } \\
\text { available }\end{array}$ & Comments \\
\hline & & & & & & $\begin{array}{l}\text { that the indicator is strongly dependent on } \\
\text { how the health system is organised. }\end{array}$ \\
\hline Finland & Hospital Discharge Register & & & & & $\begin{array}{l}\text { Number of cases was } 5912 . \text { Out of these } \\
4326 \text { were admitted from ER's. Others were } \\
\text { via referrals. }\end{array}$ \\
\hline Italy & $\begin{array}{l}\text { Ministry of Health - National } \\
\text { discharges database }\end{array}$ & & & & $2001+$ & $\begin{array}{l}\text { We consider as "cardiac procedure codes" all } \\
\text { ICD } 9 \mathrm{CM} \text { codes between } 35 .{ }^{* *} \text { and } 39 .^{* *} \text {. }\end{array}$ \\
\hline Japan & Patient Survey & $20+$ & $20+(102$ million $)$ & & & $\begin{array}{l}\text { Since the Patient Survey covers only one } \\
\text { month, the numerator (estimated } 8400 \text { ) was } \\
\text { multiplied by } 12 \text {. According to the Patient } \\
\text { Survey, the number of discharges in } \\
\text { September } 2005 \text { over } 20 \text { years with primary } \\
\text { diagnosis of hypertension was estimated as } \\
\text { approximately } 7100 \text { (subject to wide margin } \\
\text { of error inherent in sampling survey). } \\
\text { Multiplied by } 12 \text {, the annual number of } \\
\text { discharges is estimated to be } 85200 \text {. Divided } \\
\text { by Japan's population over } 20 \text { as of October } \\
2005 \text { [ } 103.56 \text { million], the rate was calculated } \\
\text { as } 82.25 / 100000 \text { annually. }\end{array}$ \\
\hline Mexico & $\begin{array}{l}\text { SIAIS-Sistema de Información } \\
\text { de Atención Integral a la } \\
\text { Salud. IMSS (DTIES) División } \\
\text { Técnica de Información } \\
\text { Estadística en Salud }\end{array}$ & $20+$ & 20 years and older & $\begin{array}{l}\text { Work with OCD-10 Codes (Unofficial } \\
\text { table ICD-9 and ICD } 10 \text { codes) }\end{array}$ & & Nationwide representative \\
\hline Netherlands & $\begin{array}{l}\text { National Medical Registry, } \\
\text { owned by PRISMANT. } \\
\text { Calculations and methods by } \\
\text { Statistics Netherlands in } \\
\text { cooperation with Institute for } \\
\text { Public Health and the } \\
\text { Environment }\end{array}$ & & & $\begin{array}{l}\text { Secondary diagnoses excluded from } \\
\text { the numerator: health problems } \\
\text { because of pregnancy, childbirth or } \\
\text { puerperium; ICD-9 codes: } 630-676 \text { or } \\
\text { V22-V24, V27, V28. Any cardiac } \\
\text { procedures; CMSV } 2.6 \text { codes: } 1273- \\
1278,1580,5350-5357,5359-5363 \text {, } \\
5369-5379,8520,8576,8640-8641 \text {, } \\
8643-8644,8649-8658,8660,8837 .\end{array}$ & & $\begin{array}{l}\text { Benign hypertension (ICD-9-CM code 401.1) } \\
\text { is not included in the OECD-selection, while } \\
\text { benign hypertensive heart disease without } \\
\text { heart failure or renal failure is (codes } 402.10 \\
\text { and } 403.10 \text { ). At codes } 403 \text { and } 404 \text { the } \\
\text { Netherlands Medical Registry does not } \\
\text { differentiate between hypertension with and } \\
\text { without heart failure and renal failure. } \\
\text { Consequently, we selected the following } \\
\text { hypertension diagnoses: } 401.0,401.9,\end{array}$ \\
\hline
\end{tabular}


DELSA/HEA/WD/HWP(2007)4

\begin{tabular}{|c|c|c|c|c|c|c|}
\hline Country & Source & $\begin{array}{l}\text { Age } \\
\text { groups } \\
\text { included }\end{array}$ & $\begin{array}{l}\text { Deviations from the definition of } \\
\text { the denominator }\end{array}$ & $\begin{array}{l}\text { Deviations from the definition of } \\
\text { the numerator }\end{array}$ & $\begin{array}{l}\text { Additiona } \\
\text { I years } \\
\text { available }\end{array}$ & Comments \\
\hline & & & & & & $\begin{array}{l}\text { 402.00, 402.10, 402.90, 403, 404. Are } \\
\text { excluded: } 401.1,402.01,402.11,402.91 \text {. We } \\
\text { assume that MDC } 14 \text { refers to health status } \\
\text { described in Chapter } 11 \text { of ICD-9 (codes } 630- \\
676 \text { ) and several V-codes (V22-V24, V27, } \\
\text { V28). Discharges from small specialised } \\
\text { hospitals are excluded. In all rates, clinical } \\
\text { admissions (with one overnight stay or more) } \\
\text { and admissions without an overnight stay } \\
\text { (=day care) were included. Transfers are } \\
\text { included in the nominator. Consequently, } \\
\text { discharges from secondary hospitals are } \\
\text { included. }\end{array}$ \\
\hline Norway & Norwegian Patientregister & & & & & Diagnoses codes ICD-10:I10-I15 \\
\hline Portugal & DRGs & & & & & \\
\hline $\begin{array}{l}\text { Slovak } \\
\text { Republic }\end{array}$ & & & & & & $\begin{array}{l}\text { Our records do not work with four digit ICD, } \\
\text { therefore it is not possible to consider } \\
\text { exclusions }\end{array}$ \\
\hline Spain & $\begin{array}{l}\text { Hospital Discharge Minimum } \\
\text { Data Set (Conjunto Mínimo } \\
\text { Básico de Datos CMBD). } \\
\text { Ministerio de Sanidad y } \\
\text { Consumo. Instituto de } \\
\text { Información Sanitaria. } \\
\text { Registro de altas 2004. }\end{array}$ & & $\begin{array}{l}\text { Information not available on } \\
\text { transferred patients }\end{array}$ & & & $\begin{array}{l}\text { Minimum data set for in-patients discharge } \\
\text { does not include data about if the patient has } \\
\text { been transferred or not from other centre. }\end{array}$ \\
\hline Sweden & $\begin{array}{l}\text { The Swedish Hospital } \\
\text { Discharge Register }\end{array}$ & & & & & \\
\hline
\end{tabular}


DELSA/HEA/WD/HWP(2007)4

\begin{tabular}{|c|c|c|c|c|c|c|}
\hline \multicolumn{7}{|c|}{ Non-OECD EU countries } \\
\hline Country & Source & $\begin{array}{l}\text { Age } \\
\text { groups } \\
\text { included }\end{array}$ & $\begin{array}{l}\text { Deviations from the definition of } \\
\text { the denominator }\end{array}$ & $\begin{array}{l}\text { Deviations from the definition of } \\
\text { the numerator }\end{array}$ & $\begin{array}{l}\text { Addition } \\
\text { al years } \\
\text { available }\end{array}$ & Comments \\
\hline Latvia & $\begin{array}{l}\text { Health statistics and } \\
\text { medical technology state } \\
\text { agency }\end{array}$ & & & $\begin{array}{l}\text { Numerator is hospital statistics rate, } \\
\text { not in primary care. Hospitalised } \\
\text { discharges of age } 18 \text { years and } \\
\text { older with hypertensive diseases } \\
\text { (ICD-10 I10, I15). }\end{array}$ & & $\begin{array}{l}\text { At this moment, we have only information } \\
\text { about discharged rate, but in future it will } \\
\text { be available from the Management } \\
\text { Information System. }\end{array}$ \\
\hline
\end{tabular}


Table 34. Hypertension admission rate, comparability issues

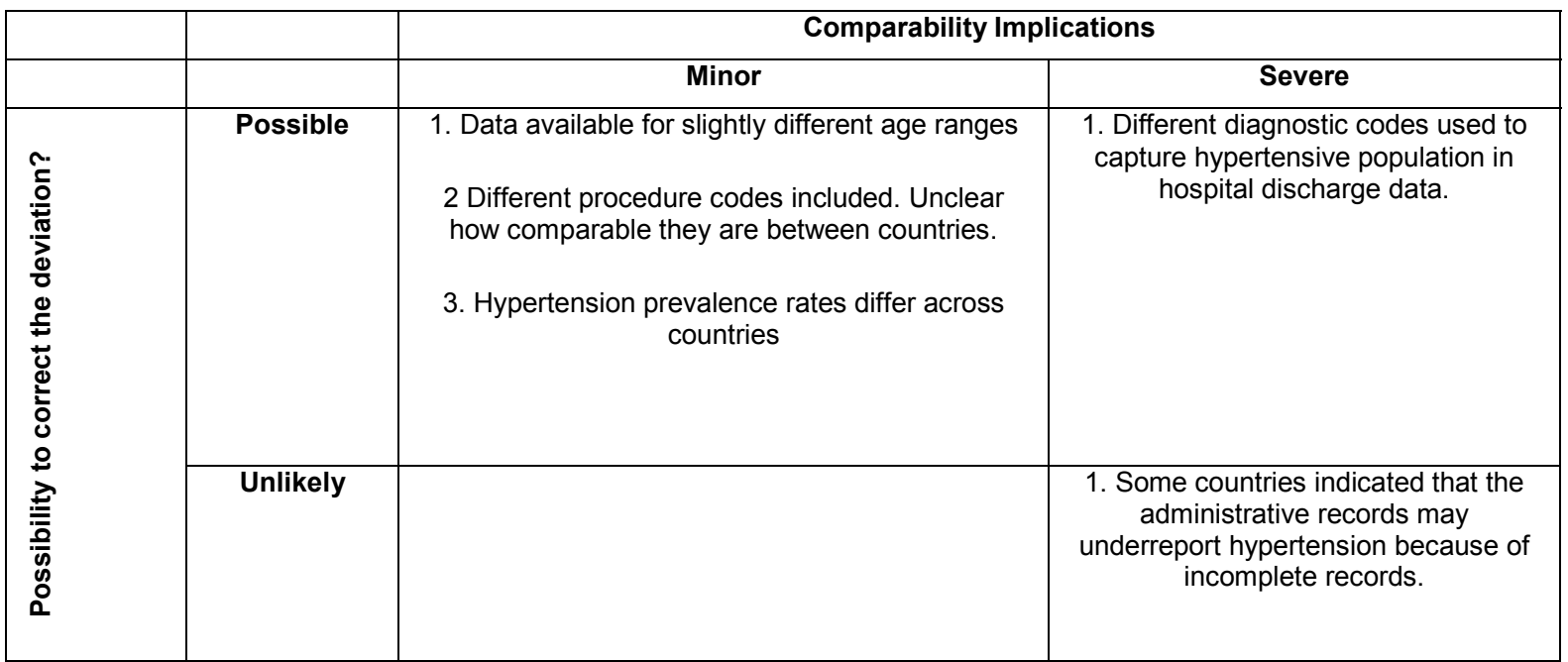

Possible solutions:

- Footnotes can indicate the year and age deviations

- Footnote (or drop if serious) if there are concerns that administrative data underreport hypertension.

- OECD will need to work with countries to ensure that comparable codes are used to calculate this indicator.

- Also age standardisation and adjustment by hypertension prevalence rates when reporting indicator 


\section{REFERENCES}

AAFA - Asthma and Allergy Foundation of America (2006), Asthma Facts and Figures, available at www.aafa.org (accessed on December 6, 2006).

Agency for Healthcare Research and Quality (2006), Guide to the Prevention Quality Indicators, AHRQ, Rockville.

American Diabetes Association (ADA) Position Statement: Implications of the United Kingdom Prospective Diabetes Study, Diabetes Care 24(1) supplement, ADA Clinical Practice Recommendations 2001, http://www.diabetes.org/clinicalrecommendations/Supplement101/S28.htm.

ADA (2003), "Economic Costs of Diabetes in the US in 2002", Diabetes Care, Vol. 26, pp. 917-932.

ADA (2006), "Summary of revisions for the 2006 Clinical Practice Recommendations", Diabetes Care, Vol. 29, Supplement, pg.1:S3.

Anderson, G.M. (1996), "Common conditions considered sensitive to ambulatory care” in Goel V, J.I. Williams, G.M. Anderson, P. Blackstein-Hirsch, C. Fooks, C.D. Naylor, (eds.), Patterns of Health Care in Ontario, 2nd Edition, Canadian Medical Association, Ottawa.

Baele, P.L., M. Bruyere, V. Deneys, V., E. Dupont, J. Flament, M. Lambermont, D. Latinne, L. Steensens, B. Van Camp and H. Waterloos, (1994), "Bedside transfusion errors: a prospective survey by the Belgium SAnGUIS group", Vox sanguinis, Vol.66, pp.117-121.

Billings, J., G.M. Anderson, and L.S. Newman, (1996), "Recent Findings on Preventable Hospitalizations", Health Affairs, Vol. 15, No. 3, pp.239-249.

Blais, L., P. Ernst, J.F. Boivin, and S. Suissa, (1998), "Inhaled corticosteroids and the prevention of readmission to hospital for asthma", American Journal of Respiratory and Critical Care Medicine, Vol. 158, No. 1, pg. 126-132.

Blustein, J., K. Hanson and S. Shea, (1998), "Preventable hospitalizations and socioeconomic status", Health Affairs (Millwood), Vol. 17, No. 2, pg. 177-189.

Callum, J.L., H.S. Kaplan, L.L. Merkley, P.H. Pinkerton, B. Rabin Fastman, R.A. Romans, A.S Coovadia and M.D. Reis, (2001), "Reporting of near-miss events for transfusion medicine: improving transfusion safety", Transfusion, Vol.41, pp.1204-1211.

Canadian Institute for Health Information (2004), Comparable Health and Health System Performance Indicators for Canada, the Provinces and Territories, CIHI, Ottawa, (http://www.cihi.ca/cihiweb/dispPage.jsp?cw_page=prtwg_2006_e). 
Centers for Disease Control and Prevention, (2005), "National diabetes fact sheet: general information and national estimates on diabetes in the United States, 2005", Atlanta, Department of Health and Human Services, Centers for Disease Control and Prevention.

Correa-de-Araujo R., K. McDermott and E. Moy, (2006), "Gender differences across racial and ethnic groups in the quality of care for diabetes", Womens Health Issues, Vol. 16, No. 2, pg. 56-65.

Eagle, C.J., J.M. Davies and J. Reason, (1992), “Accident analysis of large scale technological disasters applied to an anaesthetic complication", Canadian Journal of Anaesthesia, Vol.39, pp.118-122.

Ghafour, I.M., D. Allan and W.S. Foulds (1983), "Common Causes of Blindness and Visual Handicap in the West of Scotland”, British Journal of Ophthalmology, Vol. 67, No. 4, pp. 209-213.

Gu, K., C.C. Cowie and M.I. Harris (1999), "Diabetes and Decline in Heart Disease Mortality in US adults", Journal of the American Medical Association (JAMA), Vol. 14, No. 281, pp. 1291-1297.

Haffner, S.M. (2000), “Coronary Heart Disease in Patients with Diabetes”, New England Journal of Medicine, Vol. 342, pp. 1040-1042.

Halterman, J.S., H.L. Yoos, K. Sidora, H. Kitzman and A. McMullen (2001), "Medication Use and Health Care Contacts among Symptomatic Children with Asthma", Ambulatory Pediatrics, Vol. 1, No. 5, pp. 275-279.

Hogan, P., T. Dall and P. Nikolov, (2003), "Economic costs of diabetes in the US in 2002", Diabetes Care, Vol. 26, No. 3, pg. 917-932.

Houck, P.W., F.R. Whitehouse (2006), “Asthma prevention in urbanites”, Journal of Asthma, Vol. 43, No. 8, pg. 573-578.

IDF - International Diabetes Federation (2003), International Diabetes Federation Diabetes Atlas 3rd Edition, D. Gan (ed.), International Diabetes Federation, Brussels.

Iezzoni, L.I., J. Daley, T. Heeren, S.M. Foley, E.S. Fisher, C. Duncan, J.S. Hughes and G.A. Coffman, (1994), "Identifying complications of care using administrative data", Medical Care, Vol. 32, No. 7, pg. 700-715.

Iezzoni, L.I., S.M. Foley, T. Heeren, J. Daley, C.C. Duncan, E.S. Fisher and J. Hughes (1992), "A method for screening the quality of hospital care using administrative data: preliminary validation results", QRB Quality Review Bulletin, Vol.18, pp.361-371.

Institute of Medicine (2001), Crossing the quality chasm: a new health system for the 21st century, Washington, DC: National Academies Press.

King, H., R.E. Aubert and W.H. Herman (1998), "Global Burden of Diabetes, 1995-2025: Prevalence, Numerical Estimates, and Projections”, Diabetes Care, Vol. 21, No. 9, pp. 1414-1431.

Lavery, L. and J.D. Gazewood, (2000), “Assessing the feet of patients with diabetes", Journal of Family Practice, Vol. 49, No. 11 Suppl., pg. 9-16. 
Lawler, F.H. and N. Viviani, (1997), "Patient and Physician Perspectives Regarding Treatment of Diabetes: Compliance with Practice Guidelines", Journal of Family Practice, Vol. 44, No. 4, pg. 369-373.

Lichtenstein, M.L., M.R. Griffin, J.E. Cornell, E. Malcolm and W.A. Ray, (1994), "Risk factors for hip fracture occurring in the hospital", American Journal of Epidemiology, Vol. 140, No. 9, pg. 830-838.

Linden, J.V., K. Wagner, A.E. Voytovich, and J. Sheehan, (2000), “Transfusion errors in New York State: an analysis of 10 years' experience", Transfusion, Vol. 40, pp.1207-1213.

Mattke S, E. Kelley, P. Scherer, J. Hurst , and M.Gil Lapetra and the Members of the HCQI Expert Group (2006). Health Care Quality Indicators Project: Initial Indicators Report. OECD Health Working Paper No. 22, OECD, Paris.

Mattke, S., F. Martorell, P. Sharma, L.K. Morse, N. Lurie and M. Lara (2006b), "Estimating the Impact of Improving Asthma Treatment. A Review and Synthesis of the Literature", RAND Working Paper No. WR-448-MCAN.

McDonald, K., P. Romano, J. Geppert, S.M. Davies, B.W. Duncan, K.G. Shojania and A.Hansen (2002), Measures of Patient Safety Based on Hospital Administrative Data. The Patient Safety Indicators. Technical Review 5 (Prepared by the University of California San Francisco-Stanford Evidencebased Practice Center under Contract No. 290-97-0013). AHRQ Publication No. 02-0038 . Rockville, Agency for Healthcare Research and Quality.

Miller, M., A. Elixhauser, C. Zhan, and G. Meyer, (2001), "Patient Safety Indicators: Using administrative data to identify potential patient safety concerns", Health Services Research, Vol. 36 No. 6 (Part II), pp.110-132.

Mundell, E.J., (2004), "Simple Test Predicts Diabetes Amputation Success" (2004), available at: http://www.wtoctv.com/global/story.asp?s=2113336\&ClientType=printable, accessed August 3, 2004.

Murray, C.J.L., A.D. Lopez, C.D. Mathers, and C. Stein (2001), The Global Burden of Disease 2000 Project: Aims, Methods, and Data Sources. Global Programme on Evidence for Health Policy Discussion Paper No. 36, WHO, Geneva.

Nathan, D.M., (2002), "Initial management of glycemia in type 2 diabetes mellitus", New England Journal of Medicine, Vol. 347, No. 17, pg. 1342-1349.

National Committee for Quality Assurance, "Diabetes Quality Improvement Project initial measure set (final version)." http://www.ncqa.org/dprp/dqip2.htm, accessed May 2003.

National Heart, Lung, and Blood Institute/National Asthma Education and Prevention Program. Expert Panel Report 2: Guidelines for the diagnosis and management of asthma, in National Institutes of Health publication No. 97-4051, Bethesda, MD.

National Institute of Diabetes and Digestive and Kidney Diseases, (2005), "National Diabetes Statistics fact sheet: general information and national estimates on diabetes in the United States, 2005", Bethesda, U.S. Department of Health and Human Services, National Institute of Health.

O'Connor, P., J. Creager, S. Mooney, A.M. Laizner and J.A. Ritchie, (2006), "Taking aim at fall injury adverse events: best practices and organizational change", Healthcare Quarterly, Vol. 9, pg. 43-49. 
Ollendorf, D.A., J.G. Kotsanos, W.J. Wishner, M. Friedman, T. Cooper, M. Bittoni and G. Oster (1998), "Potential Economic Benefits of Lower-extremity Amputation Prevention Strategies in Diabetes", Diabetes Care, Vol. 21, No. 8, pp. 1240-1245.

Park, C.S., H.Y. Kang, I. Kwon, D.R. Kang and H.Y. Jung, (2006), "Cost-of-illness study of asthma in Korea: estimated from the Korea National Health insurance claims database", Korean Journal of Preventive Medicine, Vol. 39, No. 5, pg. 397-403.

Ramirez, J.A. (2005), "Worldwide Perspective of the Quality of Care Provided to Hospitalized Patients with Community-Acquired Pneumonia: Results from the CAPO International Cohort Study", Seminars in Respiratory and Critical Care Medicine, Vol. 26, No. 6, pg. 543-552.

Ray, N.F., M. Thamer, B. Fadillioglu and P.J. Gergan (1998), "Race, income, urbanicity, and asthma hospitalization in California: a small area analysis", Chest, Vol. 113, No. 5, pg. 1277-1284.

Reason, J. (1990), Human Error, Cambridge University Press.

Renders, C.M., G.D. Valk, S. Griffin, E.H. Wagner, J.T.M. van Eijk and W.J.J. Assendelft, (2003), "Interventions to improve the management of diabetes mellitus in primary care, outpatient and community settings (Cochrane Review) in: The Cochrane Library, Issue 2, 2002. Oxford: Update Software; New Zealand Guidelines Group, Primary care guidelines for the management of core aspects of diabetes, http://www.nzgg.org.nz/library/gl_complete/diabetes/index.cfm\#contents, accessed May 2003.

Roland, M. (2004), "Linking physicians' pay to the quality of care--a major experiment in the United Kingdom”, New England Journal of Medicine. Vol. 351, No. 14, pg. 1448-1454.

Rosen, A.K., P. Rivard, S. Zhao, S. Loveland, D. Tsilimingras, C.L. Christiansen, A. Elixhauser, P.S. Romano, (2005), "Evaluating the patient safety indicators: how well do they perform on Veterans Health Administration data?", Medical Care, Vol. 43, No. 9, pg. 873-884.

Sawicki, P.T. (2005), "Quality of health care in Germany. A six-country comparison", Medizinische Klinik, Vol. 100, No. 11, pg. 755-768.

Streja, D.A. and S.W. Rabkin, (1999), "Factors Associated with Implementation of Preventive Care Measures in Patients with Diabetes Mellitus", Archives of Internal Medicine, Vol. 159, pg. 294-302.

U.S. Department of Health and Human Services, (2000), Healthy People 2010. 2nd ed. With Understanding and Improving Health and Objectives for Improving Health. 2 vols. Washington, DC, Government Printing Office.

US Department of Health and Human Services (2005), US National Healthcare Quality Report, 2004, Rockville, MD: Agency for Healthcare Research and Quality.

Weissman, J.S., C. Gatsonis, and A.M. Epstein, (1992), "Rates of avoidable hospitalization by insurance status in Massachusetts and Maryland" JAMA, Vol. 268, No. 17, pg. 2388-2394.

Wenzel, S.E. (2006), “Asthma: Defining of the Persistent Adult Phenotypes”, The Lancet, Vol. 368, No. 9537, pp. 804-813.

Zimmet, P., K.G. Alberti and J. Shaw (2001), "Global and Societal Implications of the Diabetes Epidemic”, Nature, Vol. 414, No. 6865, pp. 782-787. 
DELSA/HEA/WD/HWP(2007)4

\section{OECD HEALTH WORKING PAPERS}

Papers in this series can be found on the OECD website: www.oecd.org/els/health/workingpapers

No. 28 PHARMACEUTICAL PRICING AND REIMBURSEMENT POLICIES IN SWEDEN (2007) Pierre Moïse and Elizabeth Docteur

No. 27 PHARMACEUTICAL PRICING AND REIMBURSEMENT POLICIES IN SWITZERLAND (2007) Valérie Paris and Elizabeth Docteur

No. 26 TRENDS IN SEVERE DISABILITY AMONG ELDERLY PEOPLE: ASSESSING THE EVIDENCE IN 12 OECD COUNTRIES AND THE FUTURE IMPLICATIONS (2007) Gaétan Lafortune, Gaëlle Balestat, and the Disability Study Expert Group Members

No. 25 PHARMACEUTICAL PRICING AND REIMBURSEMENT POLICIES IN MEXICO (2007) Pierre Moïse and Elizabeth Docteur

No. 24 PHARMACEUTICAL PRICING AND REIMBURSEMENT POLICIES IN CANADA (2006) Valérie Paris and Elizabeth Docteur

No. 23 HEALTH CARE QUALITY INDICATORS PROJECT, CONCEPTUAL FRAMEWORK PAPER (2006) Edward Kelley and Jeremy Hurst

No. 22 HEALTH CARE QUALITY INDICATORS PROJECT, INITIAL INDICATORS REPORT (2006) Soeren Mattke, Edward Kelley, Peter Scherer, Jeremy Hurst, Maria Luisa Gil Lapetra and the HCQI Expert Group Members

No. 21 THE SUPPLY OF PHYSICIANS IN OECD COUNTRIES (2006) Steven Simeons and Jeremy Hurst

No. 20 CONSUMER DIRECTION AND CHOICE IN LONG-TERM CARE FOR OLDER PERSONS, INCLUDING PAYMENTS FOR INFORMAL CARE: HOW CAN IT HELP IMPROVE CARE OUTCOMES, EMPLOYMENT AND FISCAL SUSTAINABILITY? (2005) Jens Lundsgaard

No. 19 TACKLING NURSE SHORTAGES IN OECD COUNTRIES (2004) Steven Simoens, Mike Villeneuve and Jeremy Hurst

No. 18 PRIVATE HEALTH INSURANCE IN THE NETHERLANDS. A CASE STUDY (2004) Nicole Tapay and Francesca Colombo

No. 17 SKILL-MIX AND POLICY CHANGE IN THE HEALTH WORKFORCE: NURSES IN ADVANCED ROLES (2004) James Buchan and Lynn Calman

No. 16 SHA-BASED NATIONAL HEALTH ACCOUNTS IN THIRTEEN OECD COUNTRIES: A COMPARATIVE ANALYSIS (2004) Eva Orosz and David Morgan

No. 15 PRIVATE HEALTH INSURANCE IN OECD COUNTRIES: THE BENEFITS AND COSTS FOR INDIVIDUALS AND HEALTH SYSTEMS (2004) Francesca Colombo and Nicole Tapay

No. 14 INCOME-RELATED INEQUALITY IN THE USE OF MEDICAL CARE IN 21 OECD COUNTRIES (2004) Eddy van Doorslaer, Cristina Masseria and the OECD Health Equity Research Group

No. 13 DEMENTIA CARE IN 9 OECD COUNTRIES: A COMPARATIVE ANALYSIS (2004) Pierre Moise, Michael Schwarzinger, Myung-Yong Um and the Dementia Experts' Group

No. 12 PRIVATE HEALTH INSURANCE IN FRANCE (2004) Thomas C. Buchmueller and Agnes Couffinhal

No. 11 THE SLOVAK HEALTH INSURANCE SYSTEM AND THE POTENTIAL ROLE FOR PRIVATE HEALTH INSURANCE: POLICY CHALLENGES (2004) Francesca Colombo and Nicole Tapay

No. 10 PRIVATE HEALTH INSURANCE IN IRELAND. A CASE STUDY (2004) Francesca Colombo and Nicole Tapay 
No. 9 HEALTH CARE SYSTEMS: LESSONS FROM THE REFORM EXPERIENCE (2003) Elizabeth Docteur and Howard Oxley

No. 8 PRIVATE HEALTH INSURANCE IN AUSTRALIA. A CASE STUDY (2003) Francesca Colombo and Nicole Tapay

No. 7 EXPLAINING WAITING-TIMES VARIATIONS FOR ELECTIVE SURGERY ACROSS OECD COUNTRIES (2003) Luigi Siciliani and Jeremy Hurst

No. 6 TACKLING EXCESSIVE WAITING TIMES FOR ELECTIVE SURGERY: A COMPARISON OF POLICIES IN 12 OECD COUNTRIES (2003) Jeremy Hurst and Luigi Siciliani

No. 5 STROKE CARE IN OECD COUNTRIES: A COMPARISON OF TREATMENT, COSTS AND OUTCOMES IN 17 COUNTRIES (2003) Lynelle Moon, Pierre Moïse, Stéphane Jacobzone and the ARD-Stroke Experts Group

No. 4 SURVEY OF PHARMACOECONOMIC ASSESSMENT ACTIVITY IN ELEVEN COUNTRIES (2003) Michael Dickson, Jeremy Hurst and Stéphane Jacobzone

No. 3 OECD STUDY OF CROSS-NATIONAL DIFFERENCES IN THE TREATMENT, COSTS AND OUTCOMES OF ISCHAEMIC HEART DISEASE (2003) Pierre Moise, Stéphane Jacobzone and the ARD-IHD Experts Group

No. 2 INVESTMENT IN POPULATION HEALTH IN FIVE OECD COUNTRIES (2003) Jan Bennett

No. 1 PHARMACEUTICAL USE AND EXPENDITURE FOR CARDIOVASCULAR DISEASE AND STROKE: A STUDY OF 12 OECD COUNTRIES (2003) Michael Dickson and Stéphane Jacobzone

\section{RECENT RELATED OECD PUBLICATIONS:}

OECD HEALTH DATA 2006 (2006), available in English, French, German, Italian, Russian and Spanish on CDROM (Windows 98/2000/NT/Me/XP); online version features these languages, plus Japanese

OECD REVIEWS OF HEALTH SYSTEMS - SWITZERLAND (2006)

OECD REVIEWS OF HEALTH SYSTEMS - FINLAND (2005)

HEALTH AT A GLANCE - OECD INDICATORS 2005 (2005), published biennially

LONG-TERM CARE FOR OLDER PEOPLE (2005), OECD Health Project series

HEALTH TECHNOLOGIES AND DECISION MAKING (2005), OECD Health Project series

OECD REVIEWS OF HEALTH SYSTEMS - MEXICO (2005)

TOWARDS HIGH-PERFORMING HEALTH SYSTEMS (2004), OECD Health Project series

TOWARDS HIGH-PERFORMING HEALTH SYSTEMS: POLICY STUDIES (2004), OECD Health Project series

PRIVATE HEALTH INSURANCE IN OECD COUNTRIES (2004), OECD Health Project series A DISEASE-BASED COMPARISON OF HEALTH SYSTEMS: What is Best and at What Cost? (2003)

For a full list, consult the OECD On-Line Bookstore at www.oecd.org, or write for a free written catalogue to the following address:

OECD Publications Service

2, rue André-Pascal, 75775 PARIS CEDEX 16

or to the OECD Distributor in your country 